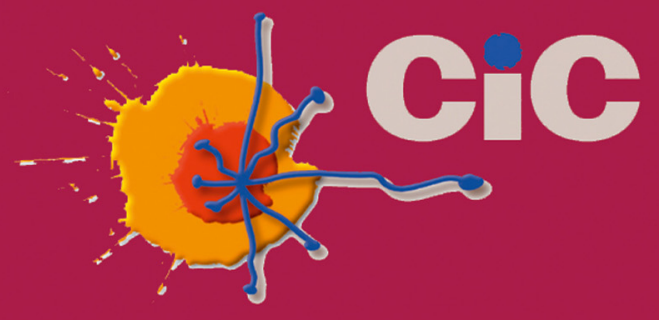

\title{
Functional and structural study of a novel membrane protein and its role in autophagy
}

Estudio estructural y funcional de una proteína transmembrana y su papel en autofagia

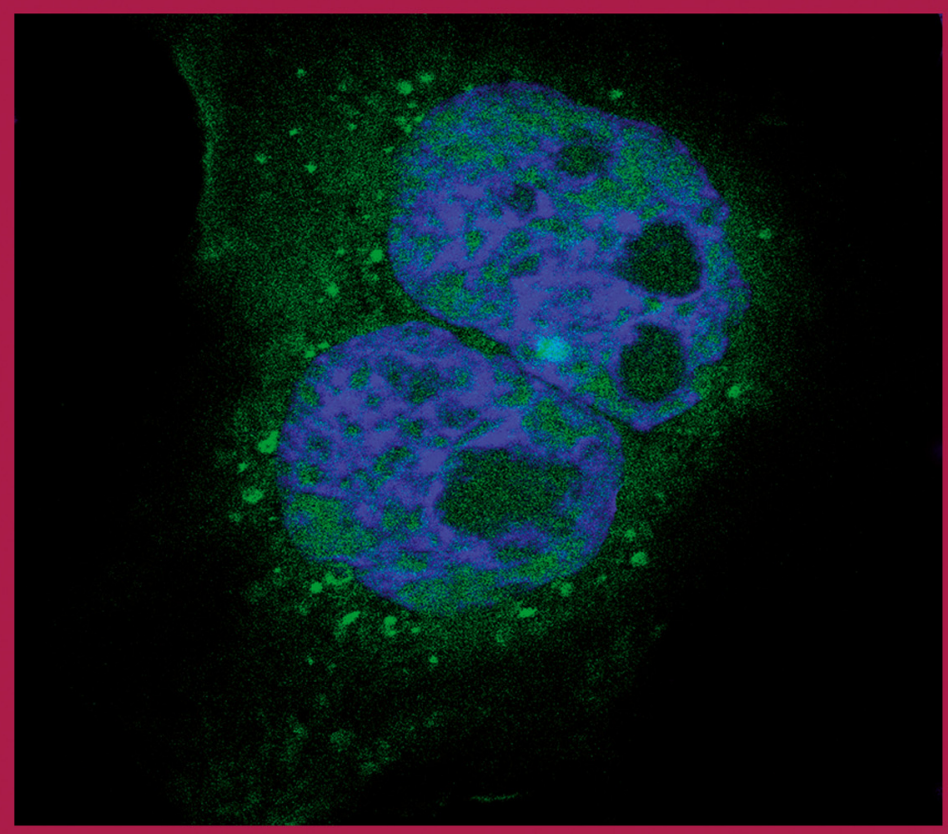

Kathrin PALLAUF

Centre of Cancer Research - University of Salamanca Centro de Investigación del Cáncer - CSIC - Universidad de Salamanca 



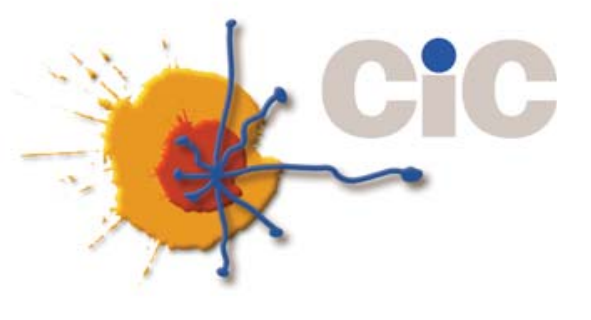

Doctoral thesis

\section{Functional and structural study of a novel membrane protein and its role in autophagy}

Estudio estructural y funcional de una proteína transmembrana y su papel en autofagia

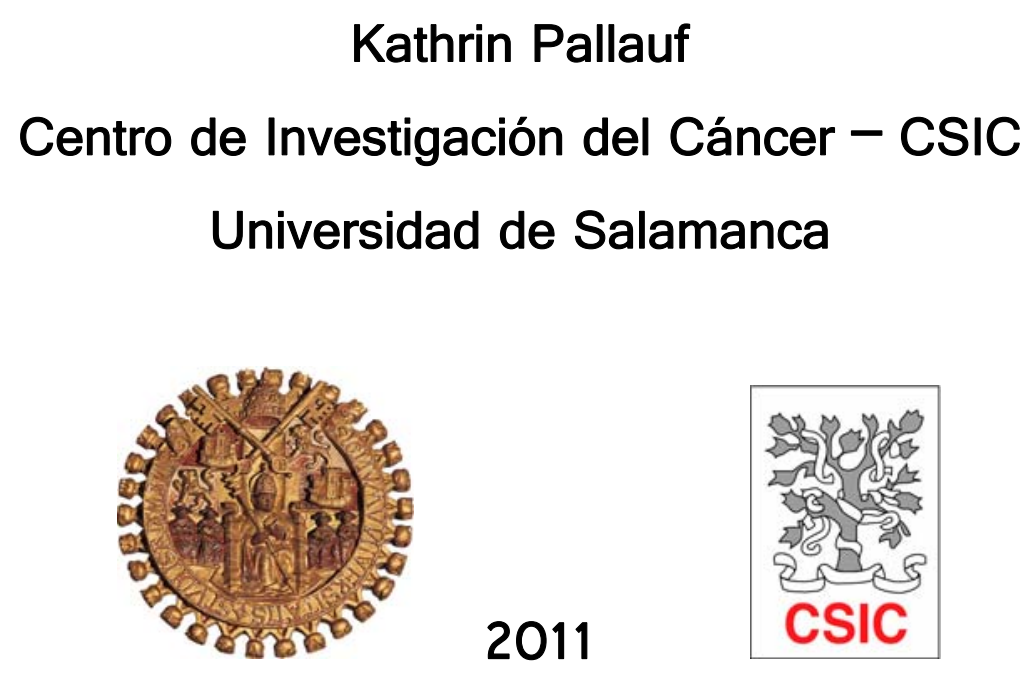


Figure on front cover: Confocal image of JAR cells transfected with BNIP3L. The green punctuate structures are formed by co-transfected GFP-LC3.

Figure on back cover: Confocal and brightfield images of an anti-AIR surface-stained SAOS-2 cell after chloroquine treatment. 
Durante mi tiempo en el CIC Salamanca he podido conocer a mucha gente que hizo posible la elaboración de esta tesis, sea por sus conocimientos científicos y su voluntad de compartirlos, sus consejos y ayuda con cualquier problema, hacer que me lo pasara bien y sintiera acogida aquí en Salamanca, o por escuchar mis penas.

En primer lugar quiero agradecer a mi director de tesis Felipe Pimentel que me diera la posibilidad de realizar este estudio sobre esta proteína interesante que me ha enganchado y fascinado. Siempre estuviste ahí para hablar de cualquier duda y dispuesto a comunicar tus conocimientos. Gracias por ello.

A Aarne, con quien he compartido casi la totalidad del tiempo de mi tesis trabajando en el laboratorio y en el mismo proyecto. Me ha beneficiado muchísimo tu trabajo, tus conocimientos y tu ayuda. Gracias por tu amistad y tu buen espíritu.

A Sonia, quién me enseño desde el principio cultivos celulares y el mundo del heavy. Siento que lo segundo no sea para mí. Gracias por haber sido tan buena compañero de laboratorio y amiga.

A Martina, mi compañera y amiga alemana, que me hizo sentir más cerca de casa y pasar buenos momentos en el laboratorio. Gracias por tu apoyo psicológico, tus consejos, por dejarme aprender de ti, los bizcochos y las risas.

A Emilio y Michal, que siguen en el laboratorio. Gracias por vuestro compañerismo, altruismo y buen rollo.

A los ex-coinquilinos del laboratorio José Pichel por su ayuda y su carácter sociable y a Nuria por enseñarme lo mejor de la fiesta salmantina.

A la Dra. Levine, por acogerme en su laboratorio durante unos meses y transmitirme muchos conocimientos valiosos para mi trabajo. 
A Cristina y Yurema por las clases de español científico, compartir gran parte del tiempo de doctorado y por pasármelo tan bien con vosotras que me dará todavía más pena irme de Salamanca.

A toda la gente del CIC que contribuyo a un ambiente de trabajo muy agradable y me hizo pasar muy buenos ratos también fuera del laboratorio, sobre todo a Laura, Lara, Janis y Dani, sin olvidar a gente que ya no está como Helena y David.

A mi compañera de biblioteca Alicia con quién compartí la carga de la fase de escritura y quién hizo junto con los Javis y los demás chicos que un rato de comedor fuera uno de los momentos más esperados del día.

Al mejor equipo de Ultimate, Quimera por sacarme de la biblioteca y algún fin de semana de Salamanca. Mi espíritu es naranja.

A mi "otro laboratorio" en bromatología por conocerme desde mis comienzos en Salamanca y regalarme una buena compañía (y alimentación) en todos los cafés, almuerzos y cenas de cuales salían buenas amistades. Gracias sobre todo a María (que llevaba razón desde el principio), Susana, Mati, Romina, Joaquín, Cristina y Montse.

A Miluska por ser buena compañera de piso, de fiesta y de charlar y por compensar la ausencia de familia cuando hacía falta.

A mi madre por corregirme el inglés y por comprender que me haya escapado a España.

Y por último a mis amigos y a mi familia de Alemania porque a pesar de la distancia han sabido hacerme llegar sus ánimos y su cariño. 
El Dr. Felipe Xosé Pimentel Muiños, Científico Titular del Consejo Superior de Investigaciones Científicas y miembro del Instituto Universitario de Biología Molecular y Celular del Cáncer (IBMCC, CSIC-USAL) de la Universidad de Salamanca,

\section{CERTIFICA:}

Que el trabajo titulado "Estudio estructural y funcional de una nueva proteína transmembrana y su papel en autofagia" presentado por Dña. Kathrin Pallauf, ha sido realizado bajo su dirección en el Centro de Investigación del Cáncer de la Universidad de Salamanca, y reúne, a su juicio, originalidad y contenidos suficientes para que sea defendido ante el tribunal correspondiente y optar al Grado de Doctor por la Universidad de Salamanca.

$\mathrm{Y}$, para que conste a los efectos oportunos, expido y firmo el presente certificado en Salamanca, a 11 de Mayo de 2011.

Fdo.: Dr. Felipe X. Pimentel Muiños 
La financiación necesaria para la realización de esta tesis doctoral ha sido aportada por el Ministerio de Ciencia e Innovación a través del Plan Nacional de Biomedicina (SAF200501208), y mediante la concesión de una beca predoctoral de Formación del Personal Investigador (BES-2006-13365). 


\section{Table of contents}

1. Autophagy ..................................................................................................11

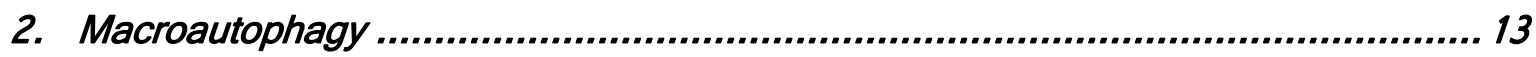

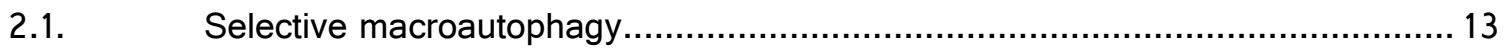

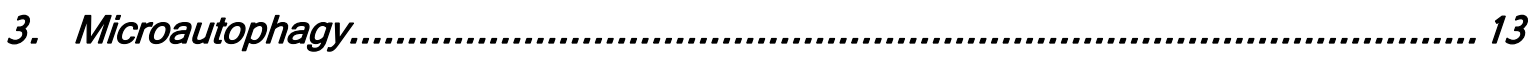

4. Chaperone-mediated autophagy ...................................................................... 14

5. Molecular mechanisms of macroautophagy -Signalling pathways and complexes- 15

5.1. MTOR

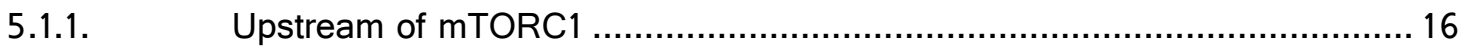

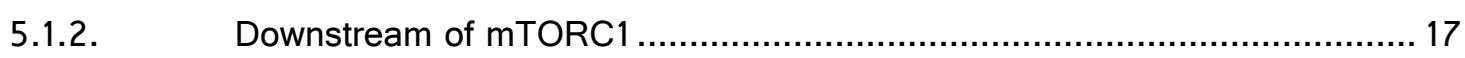

5.1.3. Regulation of autophagy via mTORC1 ............................................ 18

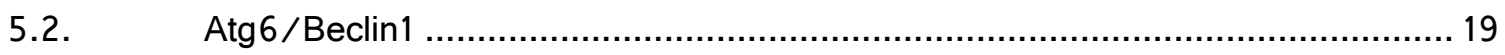

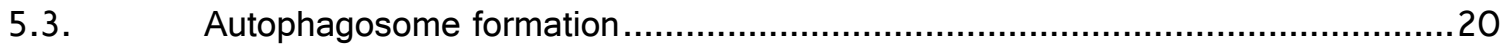

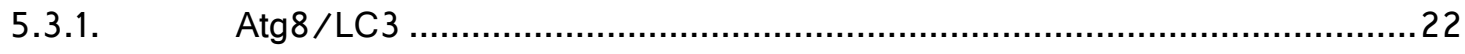

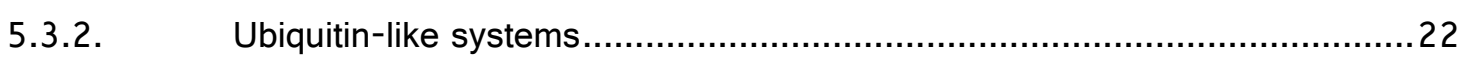

5.3.3. Atg-gene knockout and its implications for autophagy research in vitro ......23

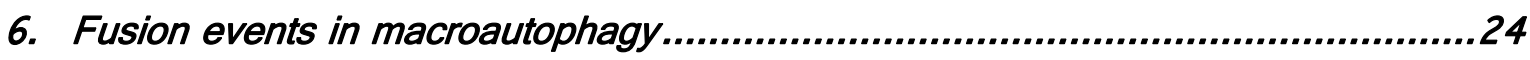

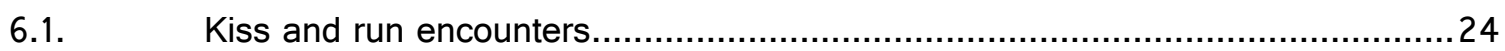

6.2. Molecular mechanisms in autophagosome fusion .......................................... 24

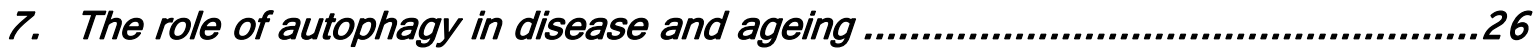

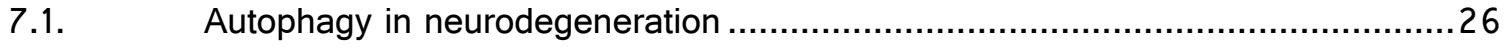

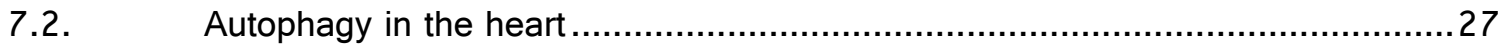

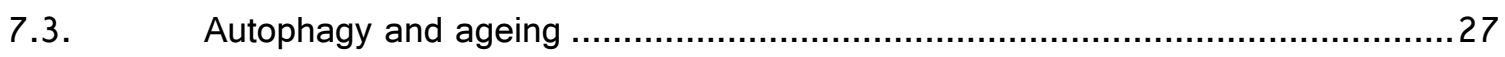

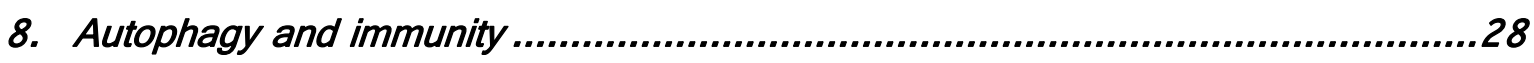

8.1. Targeting of microbes to autophagosomes (xenophagy) ................................28

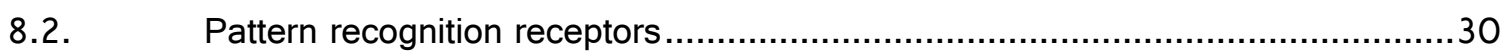

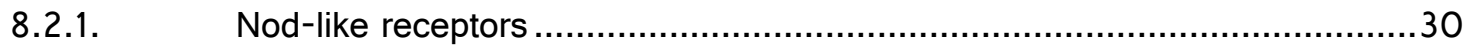

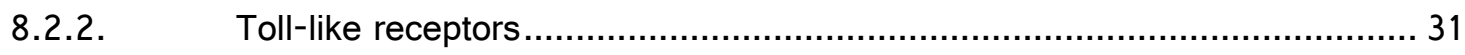

8.2.2.1. Autophagy upstream of TLR-signalling ......................................... 31

8.2.2.2. Autophagy as a consequence of TLR signalling and its role in

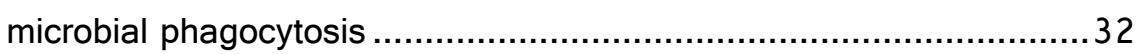

8.2.3. Retinoic acid-inducible gene I (RIG-I)-like helicases .................................33

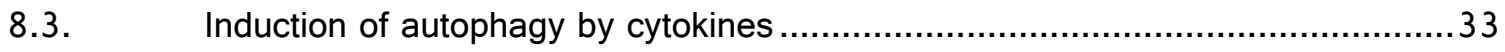

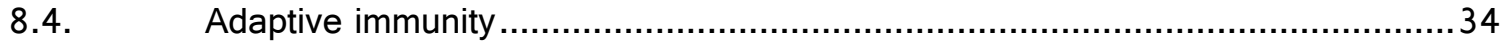




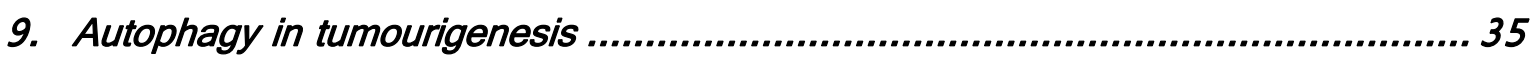

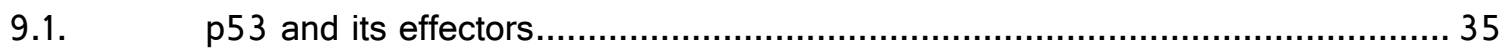

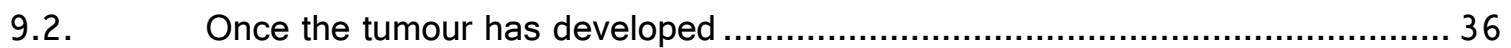

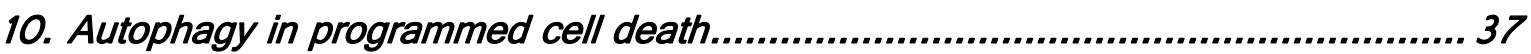

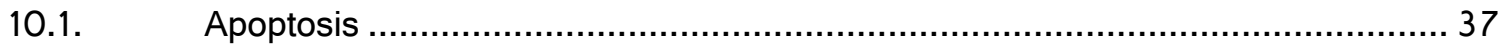

10.1.1. The two apoptosis pathways (extrinsic and intrinsic) ............................... 38

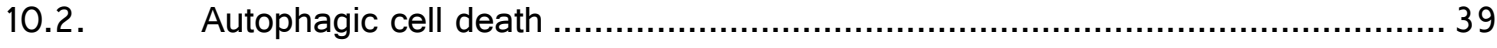

10.3. Does autophagic cell death exist? .............................................................. 41

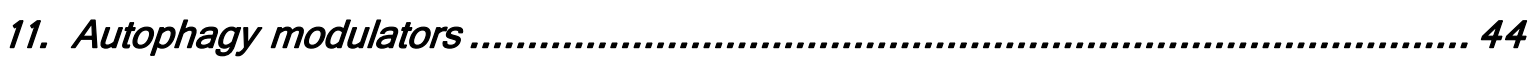

11.1. Autophagy inducers and their relevance for therapy ..................................... 44

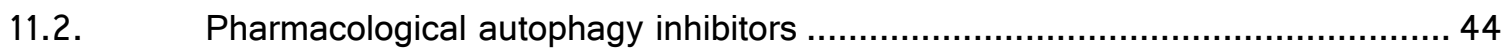

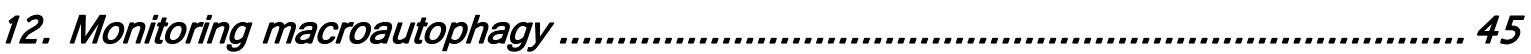

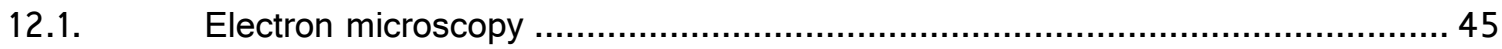

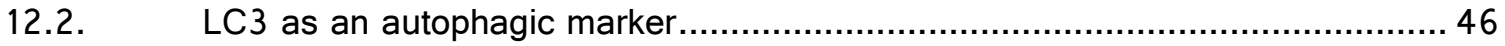

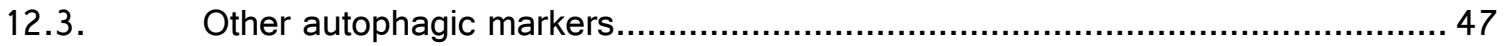

13. High-throughput screening for cell death inducers (Alcala et al. 2008)............... 49

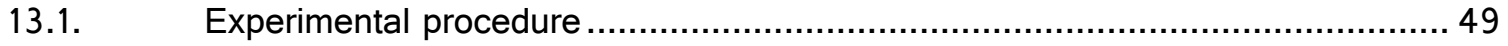

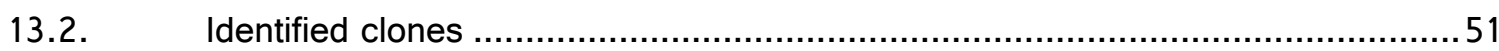

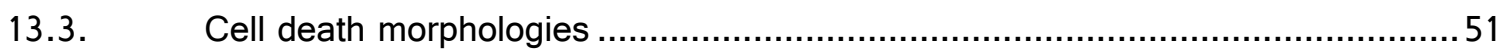

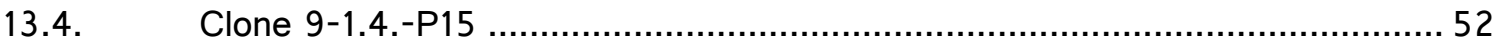

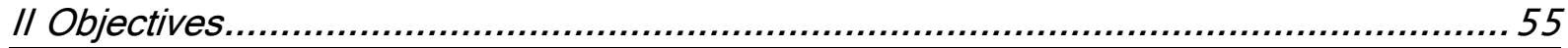

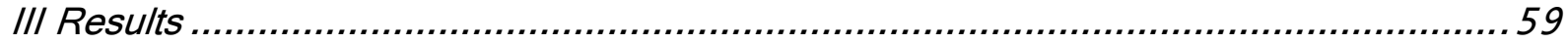

1. The clone 9-1.4.-P15 induces atypical cell death ............................................61

1.1. Morphology of the death process induced by the novel protein...........................61

1.2. Caspase-independence of the death process induced by the novel protein .......61

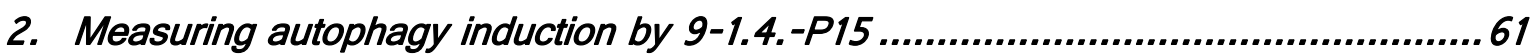

3. 9-1.4. -P15 encodes for a transmembrane protein .........................................63

4. A tagged version of $A / R$ is found on the cell surface ...................................... 64

5. Conservation of $A I R$ in other species.............................................................67

6. The clone 9-1.4.-P15 induces autophagy not only in HEK-293T cells ................ 67

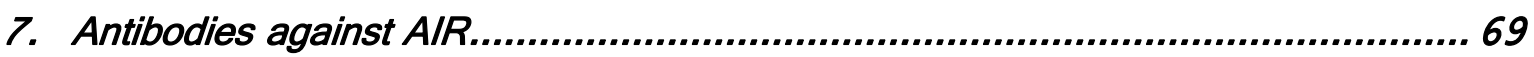

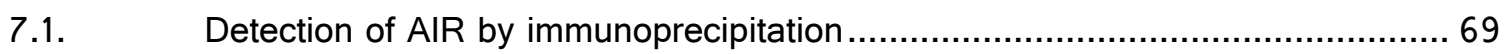

7.2. Detection of AIR by immunofluorescence ....................................................... 70

8. Subcellular localisation of AIR ...................................................................... 72

8.1. Co-stainings of AIR and cell compartment markers ....................................... 72 
8.2. Endogenously expressed AIR transiently localises to the plasma membrane.....73

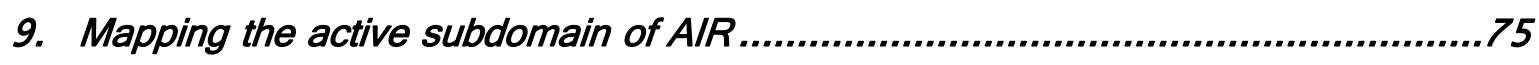

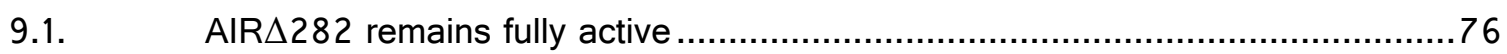

10. The intracellular domain of AIR is sufficient to induce autophagy........................78

10.1. The active subdomain of AIR is sufficient to induce autophagy .........................79

10.2. Expression levels of the different chimeras on the cell surface...........................80

10.3. Active and inactive CD16:7-AIR chimeras ................................................ 81

11. AIR and its subjection to the autophagic machinery ......................................83

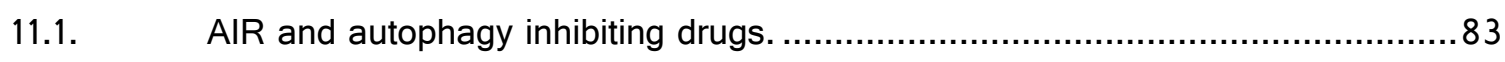

11.2. Silencing of important proteins in the macroautophagy pathway....................... 84

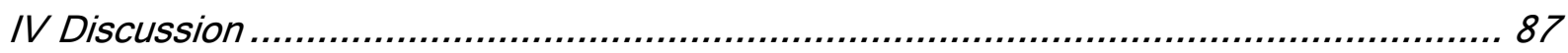

1. The previously uncharacterised protein AIR is a general inducer of autophagy.....89

2. Overexpression of proteins as a strategy for functional studies...........................89

2.1. Overexpression of AIR induces non-apoptotic cell death with autophagic features

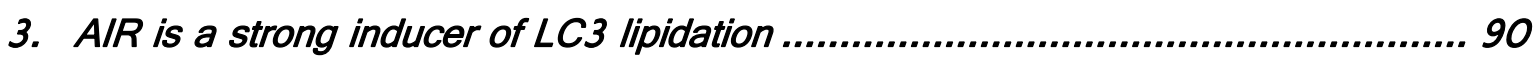

4. Evolutionary conservation of $A / R$............................................................. 90

5. Low expression levels of AIR................................................................... 91

5.1. AIR localises to the endosomal-lysosomal compartments ............................... 91

5.2. AIR is transiently located on the plasma membrane but is rapidly endocytosed. 92

5.2.1. Endocytosis contributes to autophagosome formation. .............................92

6. The intracellular part of AIR is necessary for autophagy induction ......................93

6.1. AIR $\Delta 282$ remains fully functional as an autophagy inducer .............................94

6.2. AIR could function as a transmembrane receptor..........................................94

7. The intracellular AIR domain is sufficient for autophagy induction .......................94

7.1. Structural and functional studies using CD16:7-AIR chimeras...........................95

7.1.1. Comparable surface expression of the relevant CD16:7 AIR chimeras .......95

8. AIR-263-281 is the shortest amino acid sequence of AIR-ID fully capable of inducing autophagy ............................................................................... 95

9. The active subdomain of AIR contains an endocytosis motif and is likely to be

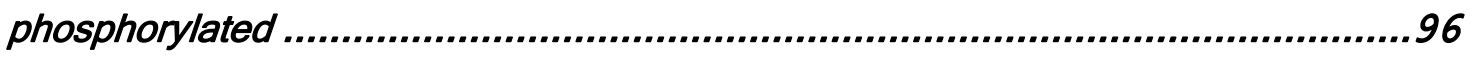

10. AIR-induced autophagy depends on the class III PI3K, Vps34.........................96

11. AIR induced autophagy depends on Atg5 and Atg7 but probably not on Beclin1 ..97

11.1. AIR does not induce Atg5/Atg7-independent autophagy ................................97

11.2. AIR could be implicated in Beclin1-independent autophagy.............................98 
12. Beclin1-independence of AlR-induced autophagy is unclear .............................. 98

12.1. GFP-LC3 in fluorescence microscopy with the chimera.................................. 99

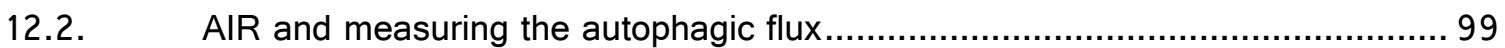

12.2.1. Inhibitors of lysosomal acidification affect AIR beyond inhibition of autophagic

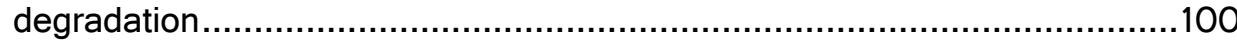

12.3. The autophagic vacuoles induced by AIR could be LC3-positive phagosomes 101

13. AIR has been reported to modulate protein glycosylation in the Golgi-apparatus 101

13.1. APP-shedding and its impact on Alzheimer's disease ..................................102

13.2. AIR as an autophagy inducer and AIR in Golgi-located glycosylation of APP

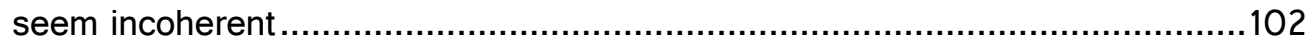

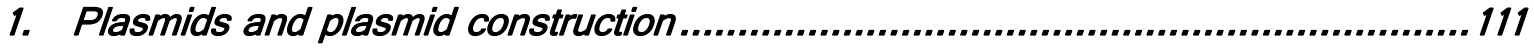

1.1. DNA amplification through polymerase chain reaction ..................................111

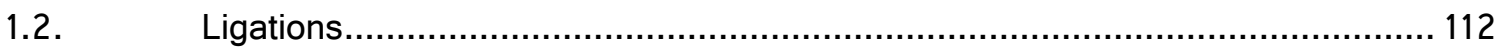

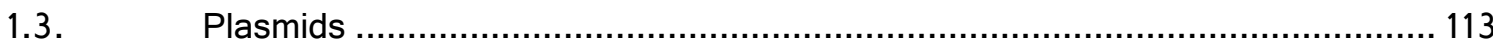

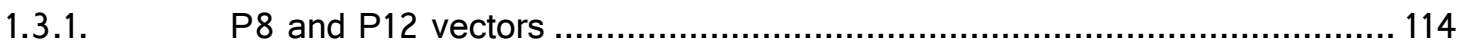

1.3.2. Vectors for retroviral transduction .................................................... 116

2. Antibodies against AlR ............................................................................ 116

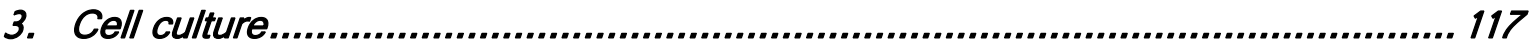

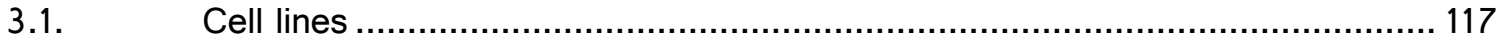

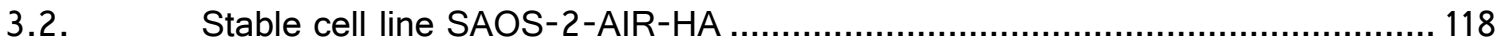

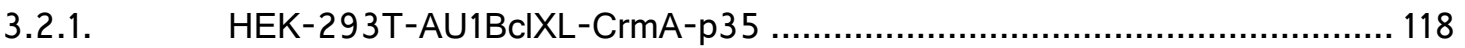

3.2.2. Generation of SAOS-2 stably expressing AIR-HA ................................. 118

4. Storage in liquid nitrogen ...................................................................... 119

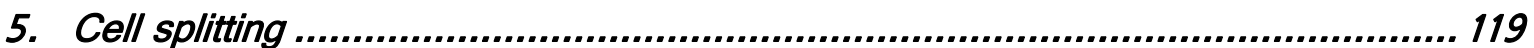

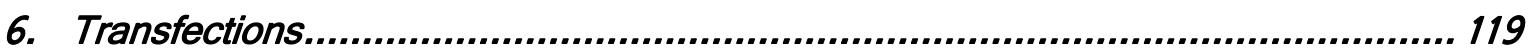

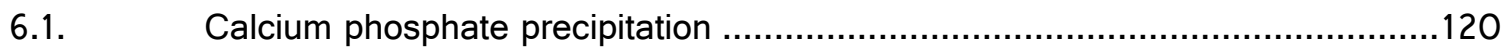

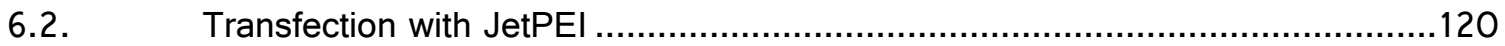

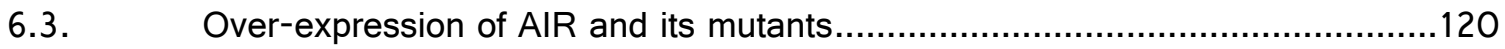

6.4. $\quad$ Aggregation of the chimera with an anti-CD16 antibody .............................. 121

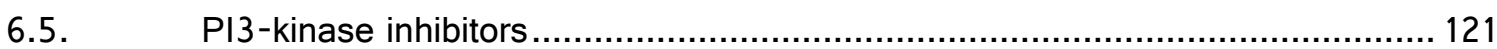

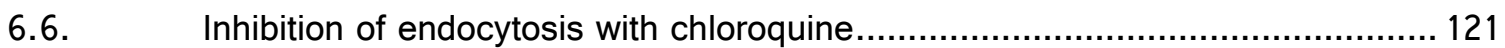

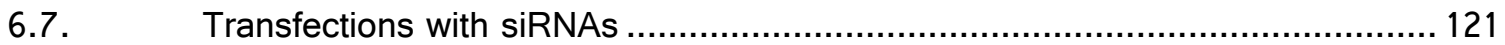

\section{Cell lysis 122}




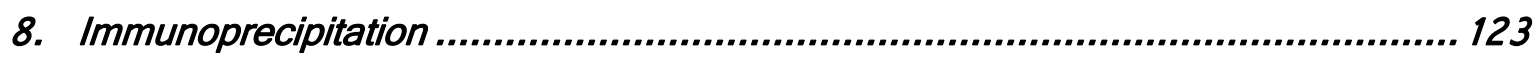

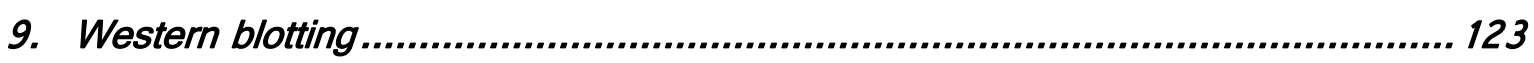

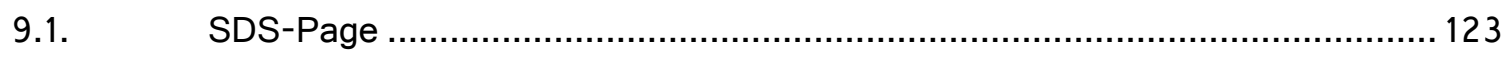

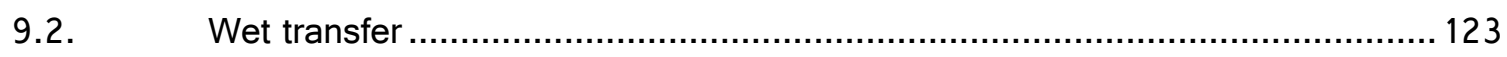

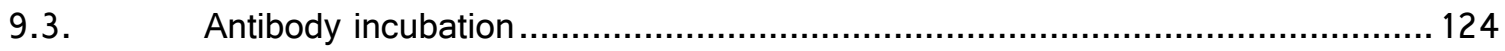

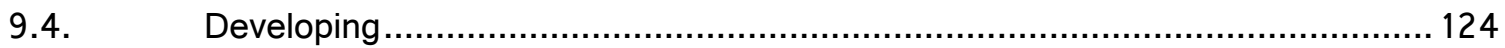

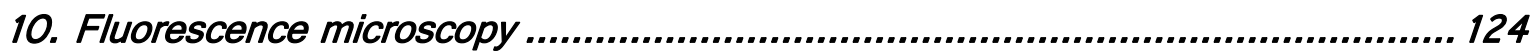

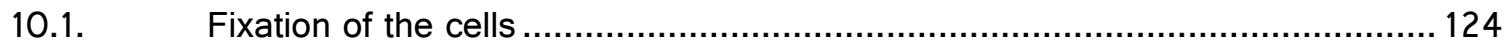

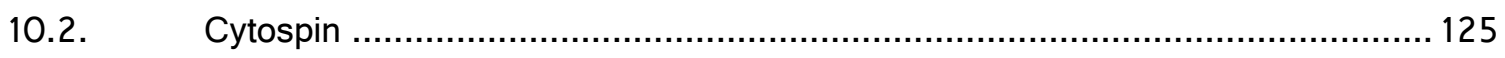

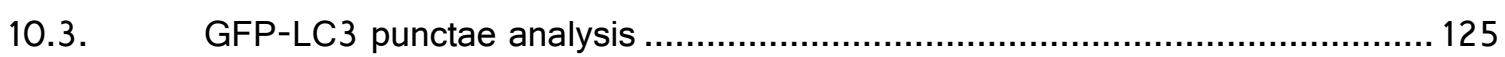

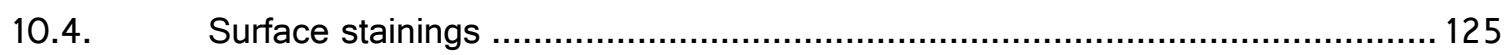

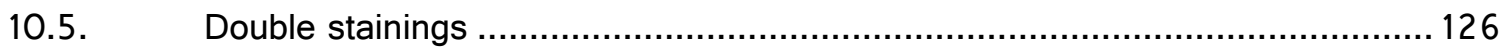

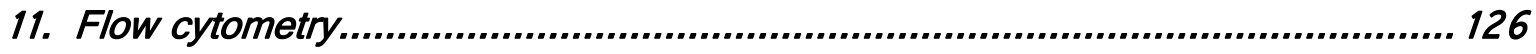

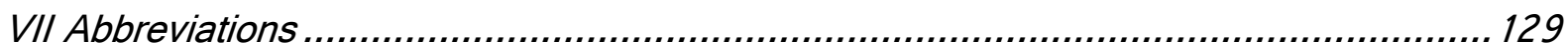

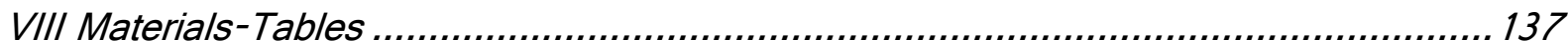

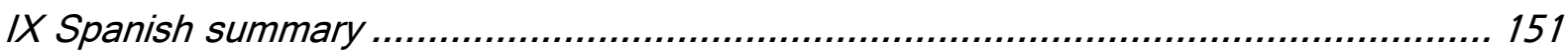

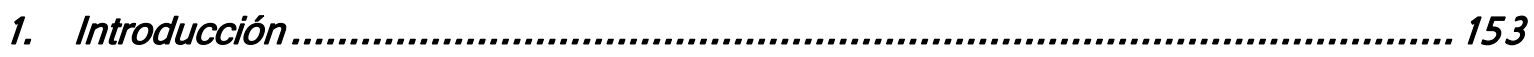

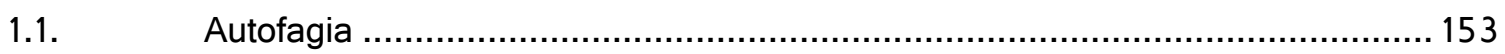

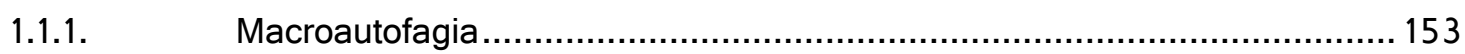

1.1.2. Autofagia mediada por proteínas chaperonas (CMA) ….......................... 155

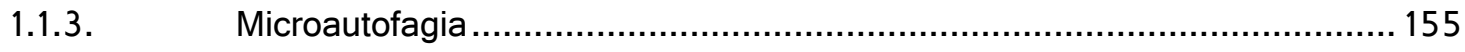

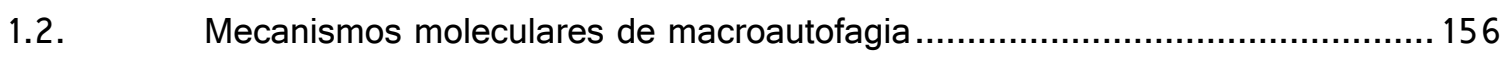

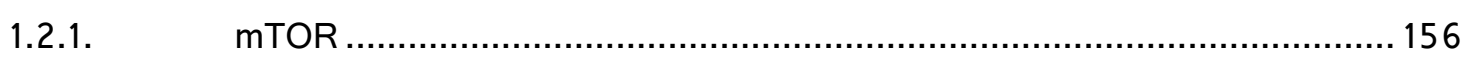

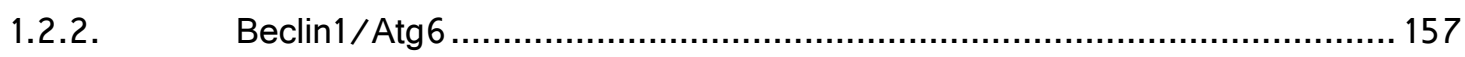

1.2.3. LC3/Atg 8 y dos sistemas de conjugación similar a la ubiquitina............... 158

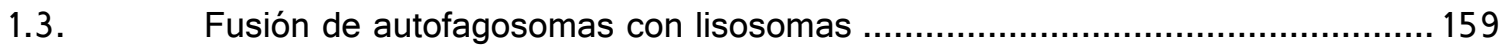

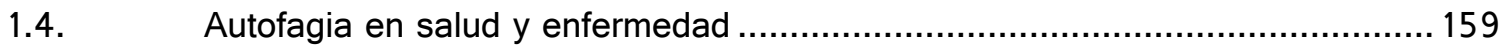

1.5. Autofagia y el sistema inmune.............................................................. 160

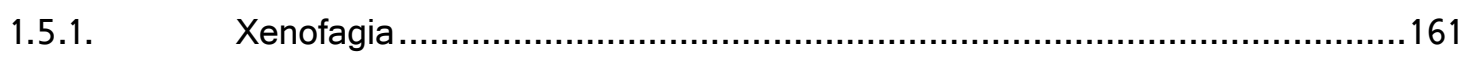

1.5.2. Receptores de reconocimiento de patrones-PRR ...................................162

1.5.3. Receptores de tipo toll-TLR ............................................................ 162

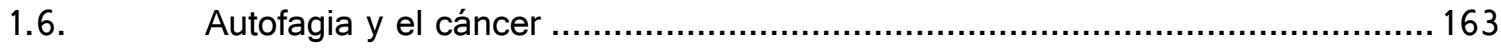

1.7. Autofagia como mecanismo de muerte celular programada ............................ 164

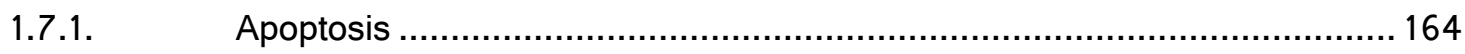

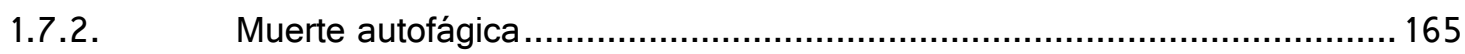




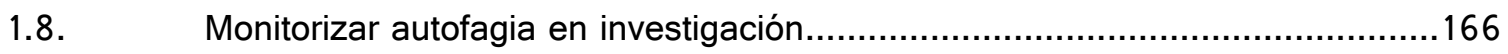

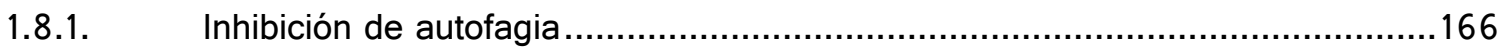

1.9. Screening de alto rendimiento para identificar inductores de muerte celular ....167

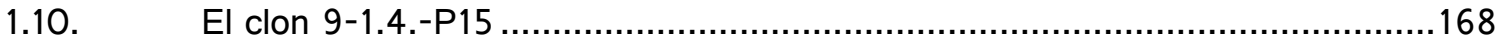

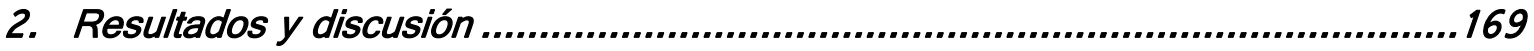

2.1. El clon 9-1.4.-P15 induce muerte autofágica ................................................169

2.2. 9-1.4-P15 codifica para una proteína transmembrana ....................................170

2.3. $\quad$ AIR es un inductor de autofagia general ................................................. 171

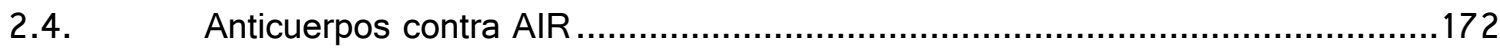

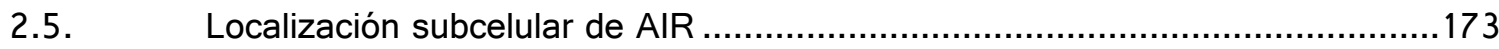

2.6. La posible implicación de endocitosis para la autofagia inducida por AIR ........173

2.7. Estudios del dominio funcional de AIR ....................................................... 175

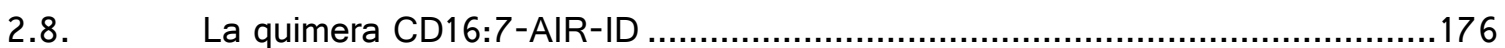

2.8.1. Presencia de las diferentes quimeras CD16:7-AIR en la membrana

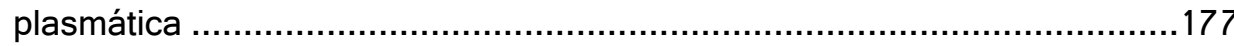

2.9. Dependencia de autofagia inducida por AIR de la maquinaria autofágica

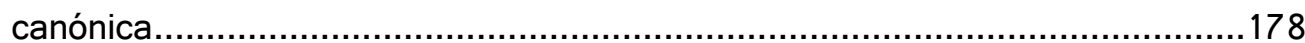

2.9.1. Silenciamiento de genes centrales de autofagia ….................................179

2.10. No se puede asegurar que AIR actúe independientemente de Beclin1 ............179

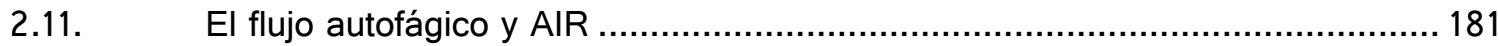

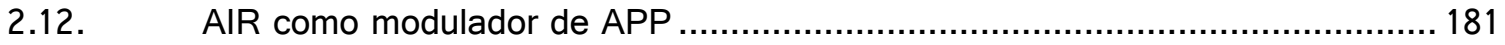

X Conclusions in Spanish............................................................................. 183

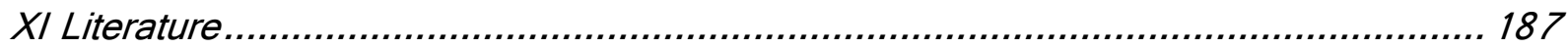

Figure 1: Different types of autophagy (modified from Mizushima et al. 2008) ............................12

Figure 2: M-tor signalling (modified from Esclatine et al. 2010, Wullschlegger et al. 2006) ........17

Figure 3: Formation and fusion of an autophagosome (modified from Cecconi et al. 2008,

Eskelinen et al. 2005).

Figure 4: Apoptosis, Autophagy-related cell death and Necrosis are the three major pathways of cell death (modified from Hotchkiss et al. 2009).................................................... 38

Figure 5: Electron microscopy (Ylä-Anttila et al. 2009) ........................................................ 46

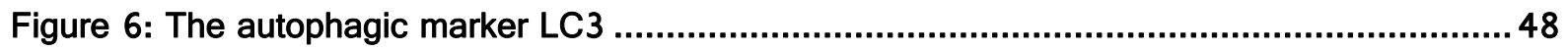

Figure 7: Detection of cell death-inducing clones (Alcalá et al. 2008) ....................................50

Figure 8: High-throughput screening for cell death inducers (Alcalá et al. 2008) ........................51

Figure 9: Different types of cell death (Alcalá et al. 2008) .....................................................52

Figure 10: Transfection of the clone 9-1.4.-P15 leads to a non-apoptotic type of cell death........63 


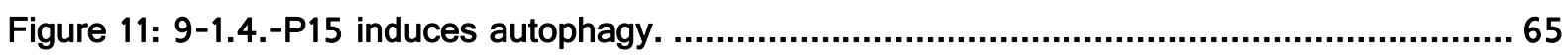

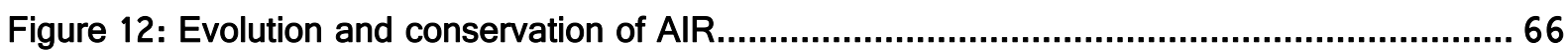

Figure 13: In HEK-293, JAR and SAOS-2 overexpressed AIR excites both isoforms of the

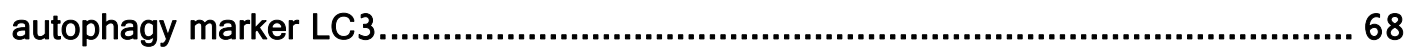

Figure 14: AIR can be detected with specific antibodies. ...................................................... 72

Figure 15: Endogenous AIR localises to late endosomes / lysosomes. ................................... 73

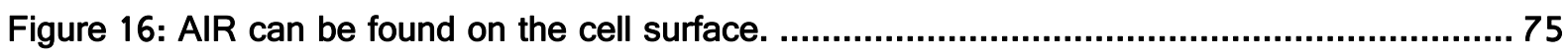

Figure 17: The intracellular part of AIR is necessary for autophagy induction in HEK-293, Jar and

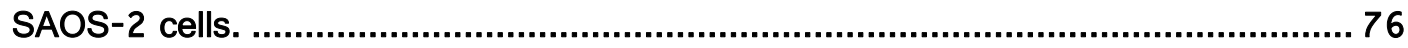

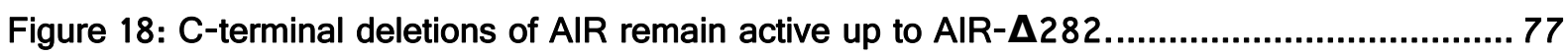

Figure 19: The chimera CD16:7:AIR-ID can be stimulated by its artificial ligand......................... 78

Figure 20: Intracellular AIR domain as part of the chimera.................................................. 79

Figure 21: The chimera with the biggest active deletion of AIR-ID CD16:7:AIR-ID-263-281 is expressed at the cell surface at levels comparable to CD16:7:AIR-full-ID. ..................81

Figure 22: The chimera CD16:7:AIR-ID-263-281 contains the active subdomain of AIR............ 82

Figure 23: The phosphoinositide 3-kinase inhibitors 3-Methyladenine (3-MA) and Wortmannin (WM) reduce AIR-induced autophagy. ............................................................ 83

Figure 24: AIR-induced LC3II formation depends on Atg5 and Atg7 but not on Beclin1............ 85

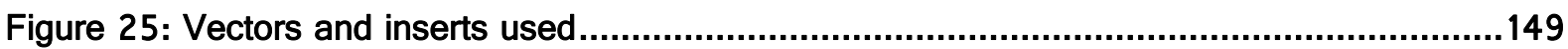

Table 1: Chemicals, enzymes and other commercially acquired material ...............................139

Table 2: Buffers prepared by the CIC sterilization unit, Salamanca or in the laboratory .............142

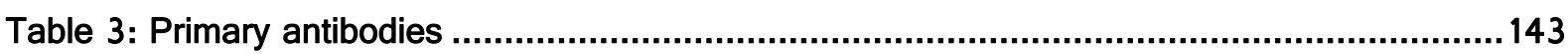

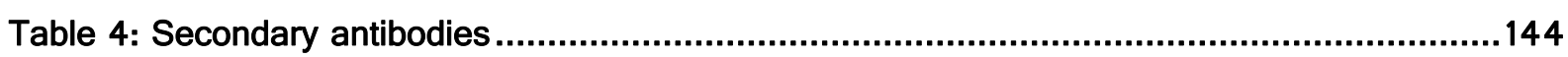

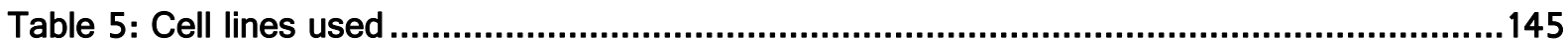

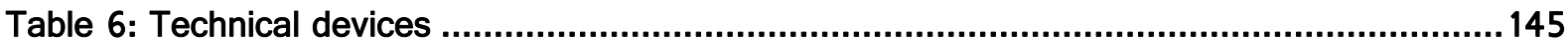

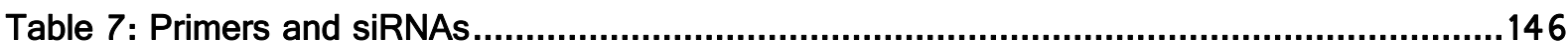





\section{Introduction}

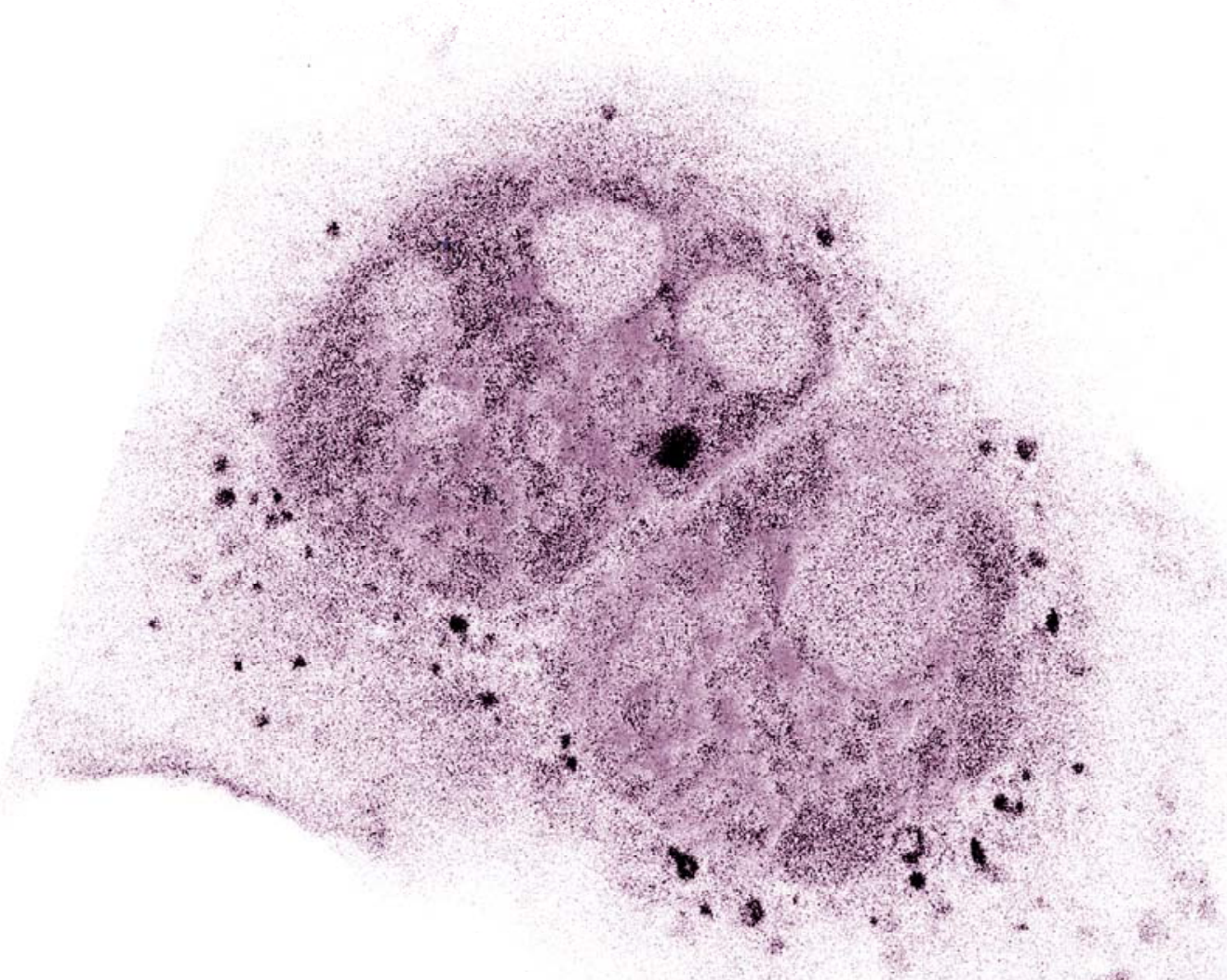





\section{Autophagy}

There are two main pathways of intracellular proteolysis, the proteasome system (Goldberg 2003) and degradation in the lysosome (De Duve et al. 1955). The proteasome system selectively degrades misfolded or damaged proteins or ones with a short half life, while the lysosome is more important for turnover of long-lived molecules. The term autophagy refers to bulk degradation of cytoplasmic components in the lysosome. Apart from soluble proteins, these components can be various types of intracellular compounds ranging from proteinaceous aggregates and lipid deposits to whole organelles or pathogens. This degradation can either be mediated by doublemembraned vesicles called autophagosomes that fuse with the lysosomes (macroautophagy), direct sequestration of small portions of cytoplasm by the lysosome (microautophagy) (Mortimore \& Schworer 1977) or chaperones which specifically recognise substrate proteins and transport them across the lysosome membrane (chaperone-mediated-autophagy) (Cuervo \& Dice 2000a). After complete degradation, the resulting minimal building blocks are transported back into the cytoplasm for recycling. For a more detailed description of the different types of autophagy, please refer to figure 1 or the following paragraphs.

The word autophagy comes from the Greek auto (oneself) and phagos (to eat) and means self-eating. As such, autophagy plays a role in cell protection but recent evidence also suggests a role in cell death. This paradox can be explained by the fact that basal levels of autophagy are important for cell homeostasis but excessive degradation of cytosolic components may be lethal (Mizushima et al. 2008). Autophagy is an evolutionarily conserved process amongst eukaryotes where it has emerged as a survival mechanism during limited nutrient supply, gaining importance in higher eukaryotes as a stress and starvation response. For example, autophagy-deficient mice die within one day of birth. The knock-out rodents that lacked key proteins for the autophagy pathway did not survive the postnatal starvation period because they could not induce this survival mechanism (Kuma et al. 2004). As the regulation of autophagy depends on amino acid and energy levels, growth factors and nutrient supplies, it is not surprising that its intensity is tightly regulated by a central kinase involved in sensing all these parameters, the mammalian target of rapamycin (m-TOR). This kinase negatively regulates multiple autophagic proteins.

Over the years autophagy has been shown to have an adaptive role in protecting organisms against diverse diseases such as neurodegenerative illnesses like Parkinson, 
Alzheimer or Huntington's disease (Komatsu et al. 2006), but it also seems to function as a tumour suppressor pathway and is important for innate and adaptive immunity (Orvedahl \& Levine 2009). The generic process termed autophagy can be divided into three different categories, macroautophagy, microautophagy and chaperone mediated autophagy, depending on the mechanisms involved.

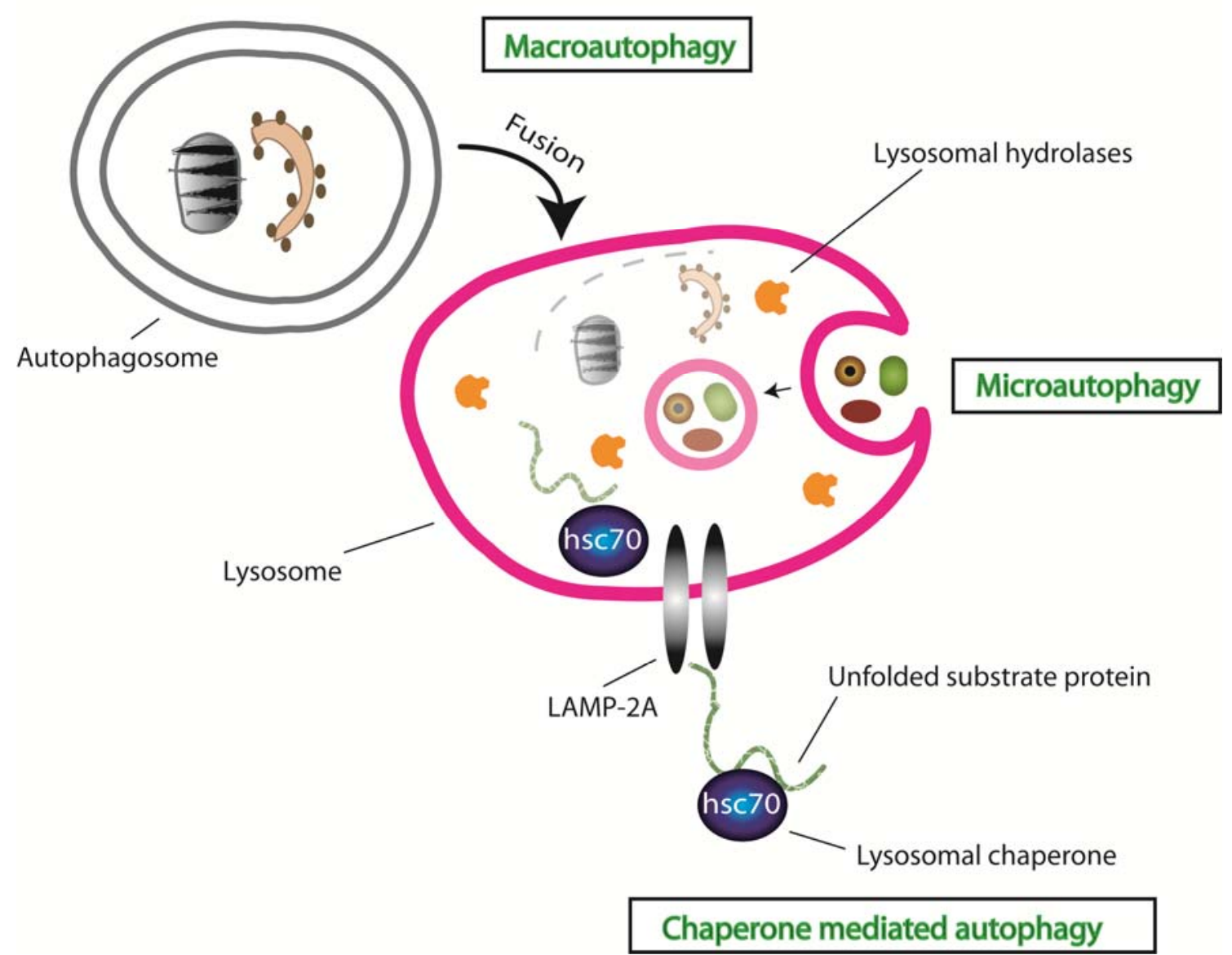

Figure 1: Different types of autophagy (modified from Mizushima et al. 2008)

Macroautophagy refers to the sequestration of cytosolic contents such as bulk cytoplasm, organelles or invading microbes by a unique double-membrane which expands from the phagophore and closes to become a vesicle called autophagosome. This autophagosome eventually fuses with the lysosome, thereby acquiring hydrolases and forming an autolysosome in which the inner membrane of the autophagosome and its contents are degraded. In a process termed microautophagy the lysosome membrane forms invaginations in which small portions of cytoplasm with cytosolic components are sequestered directly by the lysosome. In the case of chaperone-mediated autophagy (CMA), proteins are selectively unfolded and translocated through the lysosome membrane by the cytosolic and lysosomal chaperone hsc70, and the lysosomal membrane receptor LAMP-2A (lysosome-associated membrane protein type 2A). 


\section{Macroautophagy}

Macroautophagy is the most investigated type of autophagy and often simply referred to as autophagy. Upon induction of macroautophagy, vesicles with double or multiple membranes engulf portions of cytoplasm. These vesicles are called autophagosomes and eventually fuse with lysosomes to form autolysosomes in which their content is degraded. The origin of the autophagosomal membranes, that form de-novo and not through budding events, remains unknown. Recently the Golgi apparatus (van der Vaart et al. 2010), the mitochondria (Hailey et al. 2010) and the endoplasmic reticulum (Hayashi-Nishino et al. 2009) have been proposed as possible sources for their formation and expansion. It is therefore possible that these membranes originate from various organelles.

In yeast, the double membrane is formed at the phagophore assembly site (PAS). The phagophore, which is also called the isolation membrane, expands to become the double membrane which enwraps the cytosolic components and closes to form the autophagosome. It is not clear whether there is an equivalent PAS in mammals but there is certainly a phagophore which expands. A total of 33 autophagy related genes (Atg) have so far been identified in yeast and many of them have homologues in higher eukaryotes. Seventeen of these Atgs are core Atgs which are needed for macroautophagy, macropexophagy, macromitophagy and the cytoplasm-to-the-vacuoletargeting (cvt) pathway (Inoue \& Klionsky 2010).

\subsection{Selective macroautophagy}

Macropexophagy and macromitophagy are forms of macroautophagy that function by selectively degrading peroxisomes or mitochondria. However, mitochondria also seem to be degraded by non-selective bulk degradation macroautophagy.

The yeast cvt pathway shares many proteins of the macroautophagy pathway and selectively transports two vacuolar hydrolases, the aminopeptidase I (Ape1) and the $a-$ mannosidase (Ams1) from the cytoplasm to the vacuole (Scott et al. 1997; Hutchins \& Klionsky 2001). Differing from autophagy, the cvt pathway does not lead to degradation of the proteins it transports to the vacuole.

\section{Microautophagy}

Little is known about microautophagy and the majority of studies have been carried out in yeast. During microautophagy the to-be-degraded components are not sequestrated in 
vesicles prior to fusion with the lysosome or, as in the case of yeast, the vacuole, but the cytoplasmic components reach the lysosome through inward invaginations of the lysosomal membrane (Uttenweiler et al. 2005). These invaginations can be tube or finger shaped and become rather big depending on the components subjected to degradation. The tip of these invaginations is pinched off, forming a vesicle inside the lysosome which will be broken down subsequently (Muller et al. 2000). This mechanism also works for re-shaping the lysosome after fusion events with other vesicles by degrading part of the membrane (Mijaljica et al. 2007). Microautophagy is regulated by the TOR signalling complex and the EGO complex, which is associated with the vacuolar membrane (Dubouloz et al. 2005).

There are various selective microautophagy-related pathways such as micropexophagy, piecemeal nucleus autophagy and mitophagy (Todde et al. 2009). A common feature is that the target organelles are taken up by the vacuole membrane. For sequestration and degradation of peroxisomes during micropexophagy, a set of unique proteins is needed to function in coordination with the autophagy related proteins (Atg proteins) generally required for macroautophagy. For example, peroxisomes are degraded in $P$. pastoris when growth conditions change from methanol to glucose or ethanol, probably because under these conditions peroxisomal enzymes required for methanol metabolism are no longer needed.

The term "piecemeal microautophagy of the nucleus (PMN)" refers to a process in which very small parts of the nucleus are degraded by microautophagy (Roberts et al. 2003). $\mathrm{PMN}$ is up-regulated in starvation conditions and is controlled by TOR.

A possible mechanism that could contribute to the cytoprotective properties of autophagy is mitophagy, where mitochondria are selectively degraded, for instance, after they have been damaged. It has been shown that mitochondria can be selectively removed and broken down by both types of autophagy, macroautophagy (macromitophagy) and microautophagy (mitophagy) (Twig et al. 2008).

\section{Chaperone-mediated autophagy}

Whilst micro- and macroautophagy are better known for bulk degradation, chaperonemediated autophagy (CMA) is a highly selective process for the degradation of cytosolic proteins. CMA plays a role both in housekeeping and as a stress response. Prior to breaking down the proteins, the cargo needs to be recognised by chaperones and is entirely unfolded before its transport through the lysosomal membrane (Kon \& Cuervo 2010). Recognition and transport of the to-be-degraded protein are mediated by two 
major protein complexes. A cytosolic chaperone, the heat shock cognate protein of $\underline{70}$ $\mathrm{kDa}$ (hsc70) can directly recognise the protein through the motif KFERQ (Dice et al. 1990), and other co-chaperones aid in the formation of the protein complex which directs the protein to the lysosomal membrane. On arrival at the membrane a splice variant of the lysosome-associated membrane protein type 2 , LAMP-2A, allows the substrate to bind and pass over into the lysosome lumen where it is degraded within minutes. Both, LAMP$2 \mathrm{~A}$ and hsc70 are necessary for CMA; it has even been shown that the presence of LAMP-2A directly determines the rate of CMA (Cuervo \& Dice 2000b).

Whereas basal levels of CMA are detectable in all cells, this process is also induced by oxidative stress, UV light and limited nutrient supply, the latter being a factor that CMA has in common with macroautophagy. Additionally, there seems to be a crosslink between CMA and macroautophagy, as impaired macroautophagy results in activation of CMA (Kaushik et al. 2008) and vice versa (Massey et al. 2006). Under conditions of limited nutrient supply, CMA is induced after the initial starvation response by macroautophagy has decreased. CMA also plays a role in immunity, probably in MHC class II presentation, and in neurodegenerative diseases, as in the selective degradation of proteins implicated in Parkinson's disease pathogenesis.

\section{Molecular mechanisms of macroautophagy -Signalling pathways and complexes-}

The regulation of autophagy depends on signalling pathways that are also implicated in cell growth, proliferation and the decision between cell death and survival. As autophagy is an evolutionarily conserved process, most participants in its signalling pathways can be found from yeast to humans. Upstream of autophagosome formation, signalling pathways act via a Ser/Thr protein kinase called target of rapamycin (TOR) which, in turn, regulates a protein complex containing Atg1/Ulk1 involved in the early steps of autophagosome generation. Another complex involved in the autophagosome nucleation is formed around Atg6/Beclin. The membrane elongation step is driven by Atg $8 /$ LC 3 lipidation, a process which is mediated by two ubiquitin-like systems. In the following, these processes are described in more detail.

\section{1. $m T O R$}

Apart from nutrient and growth factor depletion, autophagy can also be induced by hypoxia, various drugs and radiation. As a central component of the signalling pathways 
induced by these stimuli, TOR plays an important role in macroautophagy control since TOR inhibition leads to autophagy induction. TOR was identified as a kinase that is negatively regulated by the immunosuppressor rapamycin, a drug which retards growth of mammalian cells (Wullschleger et al. 2006). Although this Ser/Thr kinase is evolutionarily conserved as a sensor in autophagy signalling, there is only one TOR in higher eukaryotes as opposed to yeast which has two homologues. This possibly explains why mammalian TOR (mTOR) can be included in two different complexes called mTORC1 and mTORC2 (Schmelzle \& Hall 2000). Whilst mTORC1 negatively regulates autophagy but stimulates transcription, translation and is sensitive to rapamycin, the lesser studied mTORC2 is important for actin organisation, and it may also have an indirect role in controlling autophagy.

Both protein complexes consist of four proteins and, besides mTOR, include the mammalian $\underline{G}$-protein $\beta$-subunit like protein (GßL, also known as $\mathrm{mLST} 8$ ). Complex 1 is also made up of the proline-rich Akt substrate $\underline{40-k D a ~(P R A S 40) ~ a n d ~ t h e ~ r e g u l a t o r y-~}$ associated protein of mTOR (raptor), whereas the second complex includes the mammalian stress-activated protein kinase-interacting protein $\underline{1}(\mathrm{mSin} 1)$ and the rapamycin-insensitive companion of mTOR (rictor).

The complex signalling events and connections between the signalling pathways of the two mTOR complexes are illustrated in figure 2. Whilst mTORC1 negatively regulates autophagy through the Ulk1/FIP200/Atg13 protein complex, which is dealt with in more detail below, the second mTOR complex, mTORC2, has been shown to influence autophagy through a mechanism which involves a transcription factor. This transcription factor, FoxO3, and its target genes LC3 and the Bcl-2/adenovirus E1B 19-kDainteracting protein 3 (BNIP3), a $\mathrm{BH} 3$-only protein, are known to induce autophagosome formation (Kanzawa et al. 2005; Esclatine et al. 2009).

\subsubsection{Upstream of mTORC1}

In its signalling pathway, mTORC1 is situated downstream of class I phosphatidylinositol $\underline{3}$-kinase $(\mathrm{PI} 3 \mathrm{~K})$ and protein kinase $\underline{B}(\mathrm{PKB})$ also known as Akt. The class I PI3K produces phosphatidylinositol bi(3,4)- and tri(3,4,5) -phosphates which activate Akt/PKB via the phosphoinositide-dependent kinase 1. In turn, Akt positively regulates mTORC1 by inhibiting the tuberous sclerosis complex $\underline{1}-\underline{2}$ (TSC 1/2) which inactivates Rheb, a

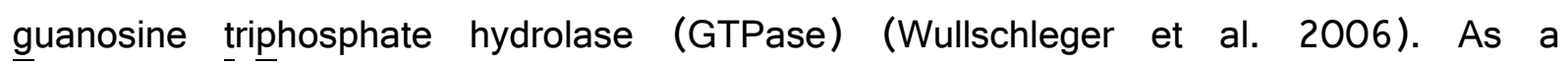
consequence of inhibiting inactivation of Rheb, the resulting activation of this GTPase also activates its direct downstream target, the mTORC1 complex, which, in turn, 
phosphorylates the ribosomal S6 kinase 1 (S6K1) and the eukaryotic initiation factor $4 \mathrm{E}$ (elF4E) binding protein 1 (4E-BP1), thus leading to protein synthesis.

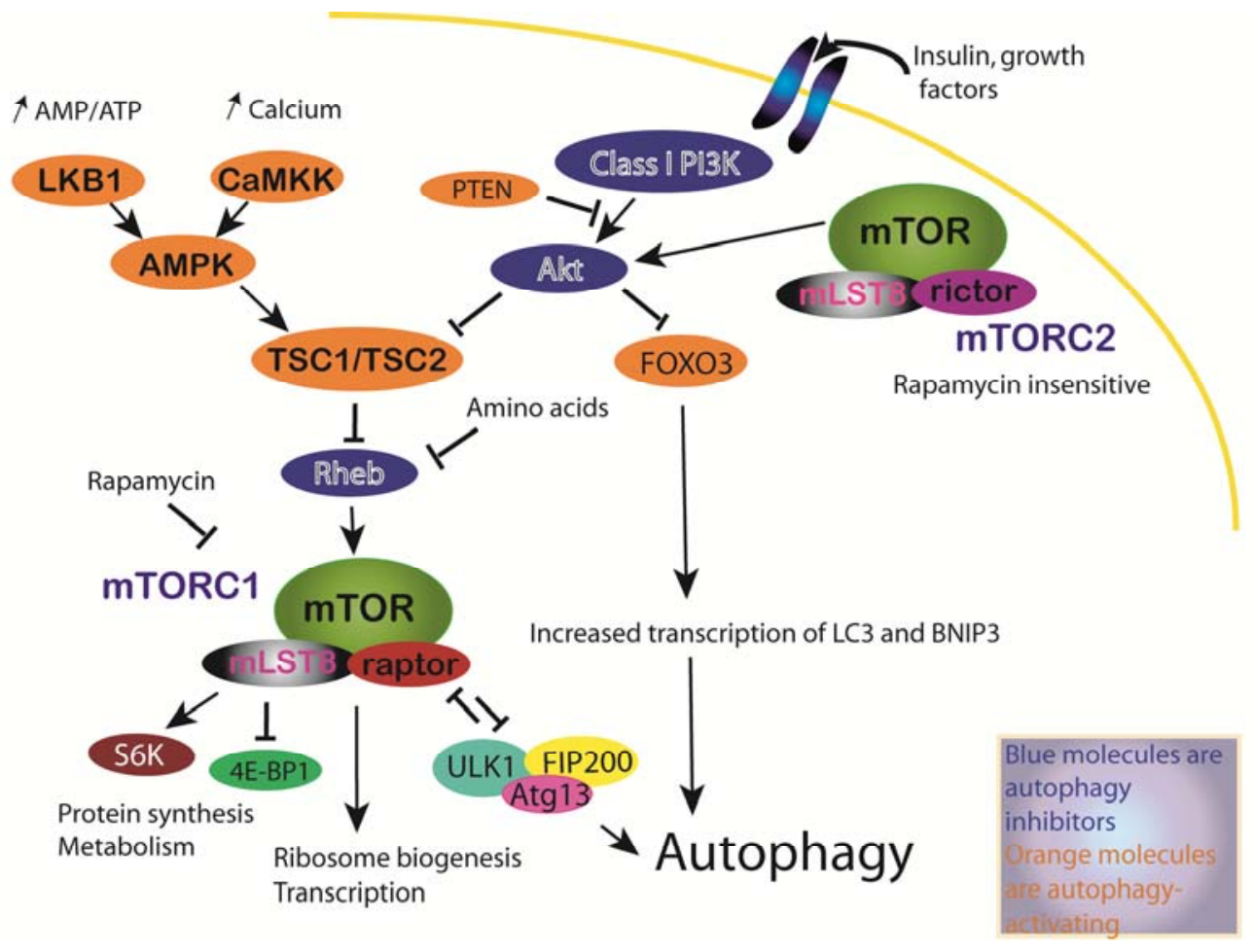

Figure 2: M-tor signalling (modified from Esclatine et al. 2010, Wullschlegger et al. 2006)

The mammalian target of rapamycin (mTOR) forms two different complexes, the rapamycin-sensitive mTORC1 and the rapamycin-insensitive mTORC2. mTORC1 negatively regulates autophagy while it induces ribosome biogenesis, transcription, protein synthesis and metabolism. mTORC1 signalling is activated (and therefore autophagy signalling is inhibited) by insulin and growth factors via a class I phosphatidyl-3-kinase which activates Akt that in turn represses the inhibiting effect of TSC1/TSC2 on the positive mTORC1 regulator, Rheb. Another protein complex formed by Ulk1, FIP200 and Atg13 is responsible for autophagy induction. This complex is negatively regulated by $\mathrm{mTORC} 1$ but also inhibits it, thereby forming a mutually antagonistic feedback loop. mTORC1 is further regulated by amino acids, AMP/ATP ratios and calcium levels, the latter two signal via the AMPK which induces TSC1/TSC2. The second mTOR complex represses autophagy in an Akt-dependent manner by inhibiting transcription of autophagy related genes through FoxO3. For further information on the nature and abbreviations of the mentioned proteins please see the main text.

\subsubsection{Downstream of $m$ TORC1}

The inhibiting effect of $\mathrm{mTORC1}$ on autophagy is mediated via the Ser/Thr protein kinase Ulk1 (the homologue of yeast Atg1) and Atg13 which binds to Ulk1 and activates it. In yeast, Atg1 needs to form a complex with Atg13 and Atg17 in order to induce autophagosome formation. Atg13 is hyperphosphorylated under nutrient rich conditions in a TOR-dependent manner and therefore loses affinity for Atg1 (Kamada et al. 2000), thus preventing autophagosome formation. In mice, mammalian Atg13 and another Ulk1binding protein, the focal adhesion kinase family-interacting protein of $200 \mathrm{kDa}$ (FIP200) have been identified as involved in this complex (Hailey et al. 2010). Despite its low 
sequence similarity, FIP200 might be the functional homologue of yeast Atg17. In mammalians the Ulk1/Atg13/FIP200 complex seems to be formed prior to phagophore induction, and Ulk1 and Atg13 are both dephosphorylated upon nutrient deprivation, suggesting that phosphorylation rather than formation of the complex is the mechanism by which mTOR influences autophagy induction (Ganley et al. 2009).

An interesting finding is that the Ulk1 complex is not only regulated by mTOR but can also signal to it. It has been shown that Ulk1 can inhibit the mTOR/S6K pathway via inhibition of S6K, one of the downstream targets of mTORC1 for protein synthesis. This study implicates a mutually antagonistic feedback loop between mTOR and Ulk1 as well as a crosstalk between autophagy and protein synthesis (Lee et al. 2007b).

\subsubsection{Regulation of autophagy via mTORC1}

Amino acids, especially leucine, also inhibit the signalling complex, and therefore mTORC1 functions as an amino acid sensor. This amino acid sensing could happen via the small GTPase Rheb which is situated upstream of mTOR and is inhibited under amino acid depletion (Roccio et al. 2006). However, there seem to be other ways to downregulate mTOR in response to limited amino acid supply whereby the exact mechanism of how mTORC1 senses amino acid levels remains unknown.

The phosphatase PTEN was initially identified as a tumour suppressor ( $\mathrm{Li}$ et al. 1997) which activates autophagy by hydrolysing Ptdlns(3,4,5)P3 (phosphatidylinositol-3phosphate) and in doing so compensates for the inhibiting effect of the class I PI3K/Akt signalling pathway (Arico et al. 2001), which lies upstream of mTOR.

Further regulators of autophagy are growth factors and insulin since they keep mTOR activated and thereby suppress autophagy. Without growth factors and insulin, mTOR is inhibited and autophagy induction rescues the cells from dying because of their being unable to absorb nutrients from outside the cells. In this scenario, autophagic degradation supplies sources for production of ATP, the titre of which needs to be kept at viable levels despite the limited nutrient supply. However, cells die after a prolonged starvation period, a demise that is most likely due to massive degradation of proteins and organelles (Esclatine et al. 2009).

The mTORC1 complex senses ATP energy levels via AMPK (adenosine monophosphateactivated protein kinase) that signals to TSC $1 / 2$. In starved cells the AMP/ATP ratio increases, leading to activation of AMPK through LKB1 (also known as serine/threonine kinase 11). Besides its role in ATP sensing, this kinase might also be a mediator for 
induction of autophagy by hypoxia through an as yet unknown mechanism (Laderoute et al. 2006).

Apart from changes in the AMP/ATP ratio, AMPK is activated through the $\mathrm{Ca}^{2+} /$ calmodulin-dependent kinase $\beta(\mathrm{CaMKK}-\beta)$ in response to an increase of free cytosolic calcium which leads to autophagy induction via mTOR (Hoyer-Hansen et al. 2007 ). Additionally, the calcium concentration seems to influence autophagy negatively in a CaMKK- $\beta / A M P K-i n d e p e n d e n t$ manner downstream of mTOR, where it affects autophagosome formation. Therefore, the overall role of calcium in autophagy signalling remains unclear; although it is believed that increased calcium levels induce autophagy at the level of AMPK, studies focussing on calcium in AMPK-independent autophagy draw different conclusions (Williams et al. 2008; Grotemeier et al. 2010).

\subsection{Atg6/Beclin1}

The events described in the following paragraphs are shown in figure 3. Human Beclin1 shares $24 \%$ homology with yeast Atg 6 and was the first mammalian gene found with a role in mediating autophagy (Liang et al. 1999). While yeast Atg6 is also implicated in vacuolar protein sorting, Beclin1 in the worm, fly and mammals only seems to contribute to autophagy. Interestingly, mouse Beclin1 has been shown to be a haplo-insufficient tumour suppressor (Qu et al. 2003). Beclin1 forms part of a complex with the class 3 phosphatidylinositol-3-kinase $\mathrm{Vps} 34$ and was first discovered as a $\mathrm{Bcl}-2$-binding protein (Liang et al. 1998). Structural studies indicate that beside the Bcl-2-binding domain (BBD), Beclin1 has a central coiled-coil domain (CCD), an evolutionarily conserved domain (ECD) and a BH3-motif- within the Bcl-2 domain (Oberstein et al. 2007). A nuclear export signal domain is responsible for its cytosolic localisation where it operates (Liang et al. 2001).

Beclin1 acts as a platform for a multiprotein complex including the class 3 PI3K, (Vps34), which produces the driving force for phagosome nucleation: phosphatidylinositol $\underline{3}-$ phosphate (Ptdlns3P). Binding to Vps34 is important for the autophagy and tumour suppressor function of Beclin1 (Furuya et al. 2005). The PI3K regulator, Vps15, forms part of this complex as well, and it seems to function at the trans-Golgi network (TGN) (Kihara et al. 2001). The magnitude of autophagy induction depends on the extent of the Beclin1-Vps34 interaction. Thus, there are various activators and inhibitors which can bind to the Beclin1/Vps34 complex and regulate its activity; for example Bcl-2 and other antiapoptotic members of its family, like $\mathrm{vBcl}-2$ and $\mathrm{Bcl}-\mathrm{xl}$ interact with the bona fide $\mathrm{BH} 3$ domain of Beclin1 and thereby inhibit it. The death-associated protein kinase (DAPK) 
regulates this process by phosphorylating Beclin1 within the $\mathrm{BH} 3$ domain, leading to dissociation of the Bcl-2 proteins and causing autophagy induction (Zalckvar et al. 2009). Another mechanism for disruption of the $\mathrm{Bcl}-2 /$ Beclin1 association is triple phosphorylation of Bcl-2 by C-Jun $\underline{N}$-terminal protein kinase 1 (JNK1), a stress-activated signalling molecule (Wei et al. 2008). The Beclin1 signalling complex is further implicated in viral pathogenicity. The herpes simplex virus type 1 confers neurotoxicity by inhibiting autophagy through its protein ICP34.5 which binds to Beclin1 as a repressor (Orvedahl et al. 2007).

A positive regulator of the Beclin1/Vps34 complex is the UV-radiation resistanceassociated gene protein (UVRAG) that binds to the coiled-coil domain of Beclin1 (Liang et al. 2006). The bax-interacting factor 1 (Bif-1, also known as endophilin B1) enhances the PI3K activity by interacting with the Beclin1 complex through UVRAG and also colocalises with Atg5, LC3 and Atg9 at the autophagosome during nutrient deprivation. UVRAG and Bif-1 are both tumour suppressor candidates (Takahashi et al. 2007). Another positive regulator of autophagy and inhibitor of cell proliferation is the activating molecule in Beclin1-regulated autophagy 1 (Ambra1) which is important for neural development in mammals (Fimia et al. 2007). Further identified activators are the vacuole membrane protein 1 (VMP1) and the small GTPase, Rab5. VMP1 co-localises with LC3 in mammals where it is necessary for autophagy and is induced by starvation and rapamycin. However, there is no known yeast homologue (Ropolo et al. 2007). Rab proteins regulate different steps in membrane trafficking and Rab5 is well-established as a regulator of early endocytosis. However, recent studies suggest that Rab5 is also involved in the early stage of autophagosome formation as it activates the PI3K in the Beclin1/Vps34 complex (Ravikumar et al. 2008). Another rab protein, Rab24 has also been shown to be implicated in autophagosome formation as it was found to localize to autophagosomes (Munafo \& Colombo 2002).

\subsection{Autophagosome formation}

Most of the core Atgs play a role in the biogenesis of the autophagosome which, due to its characteristic de novo-formed double membrane, represents a unique mechanism amongst membrane trafficking events.

As mentioned in section 2, the mitochondria, the Golgi-apparatus and the endoplasmatic reticulum have been proposed as contributing to the formation of autophagosomes. Atg9 is an integral membrane protein required for autophagosome genesis in yeast. Atg9 localises to the PAS and has been detected at other peripheral sites within the cell, for 
example at the mitochondria (Reggiori et al. 2005). As Atg9 is implicated in phagophore expansion, this finding could support the hypothesis that some autophagosomal membranes originate from mitochondria.

It is not sure whether PAS exists in mammals but, just as in yeast, there is a detectable phagophore (also called isolation membrane) which expands to become the autophagosome membrane. Most of the known Atg proteins localise to this phagophore or elongating autophagosome, amongst them, two ubiquitin-like proteins, LC3 and Atg12. While LC3 stays on the autophagosome and is eventually degraded in the autolysosome, Atg12 that forms a complex with Atg5 and Atg16L dissociates from the completed autophagosome (Cecconi \& Levine 2008).

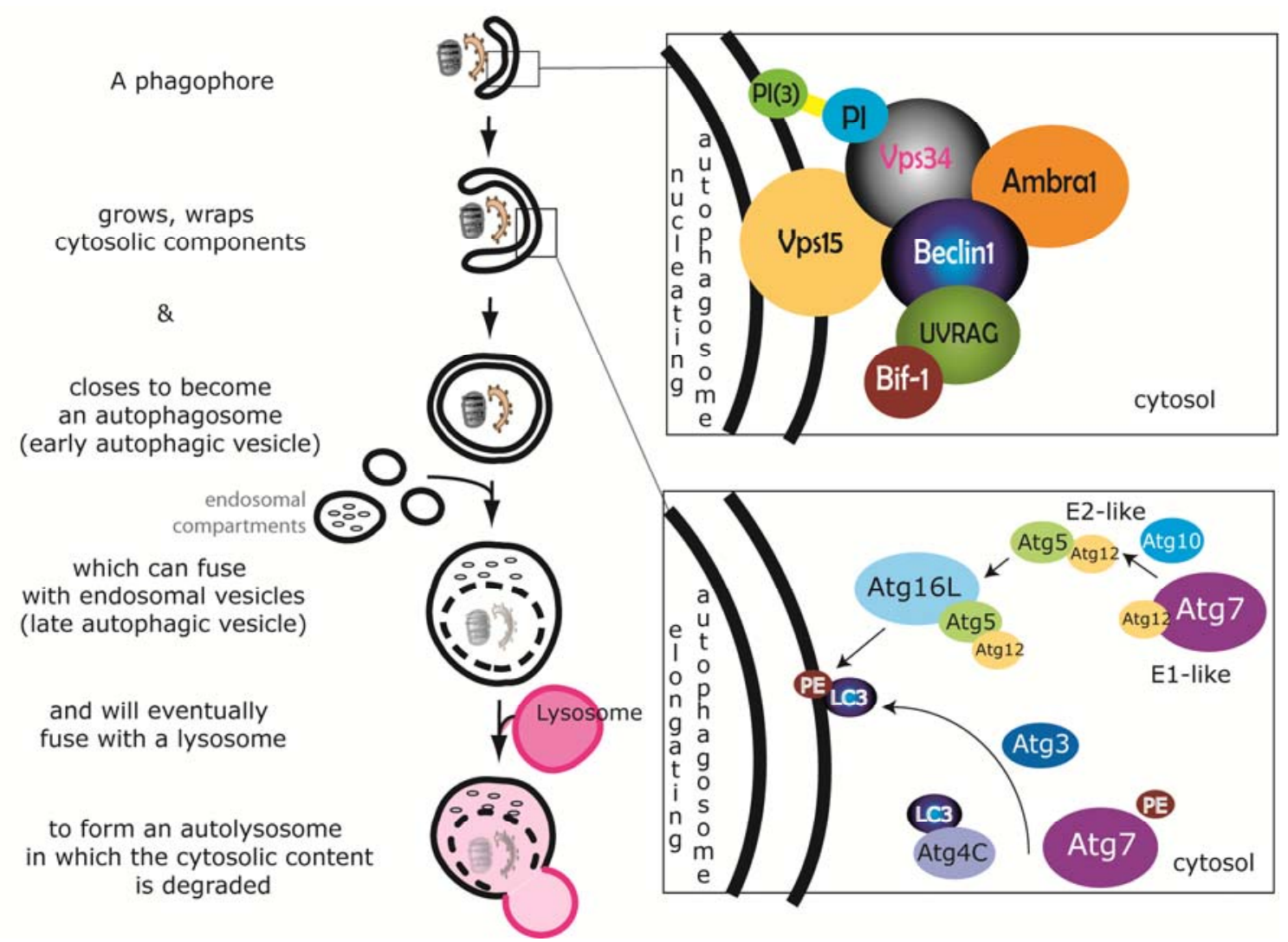

Figure 3: Formation and fusion of an autophagosome (modified from Cecconi et al. 2008, Eskelinen et al. 2005).

On the left side there is a schematic description of autophagosome formation and the subsequent fusion events which lead to degradation of the cytosolic contents. The upper box on the right side shows the molecular mechanisms involved in autophagosome nucleation. The driving force behind this process is the phosphorylation of phosphatidylinositol (PI). The responsible class 3 PI kinase, Vps 34 forms a complex with Beclin1 and Vps15. Ambra supports the binding of Beclin1 with Vps34 and Bif-1. UVRAG also regulates the complex positively. In the lower box the two ubiquitin-like conjugation systems involved in autophagosome elongation are depicted. In this process LC3 is lipidated by phosphatidylethanolamine (PE) and bound to the autophagosome membrane. Atg7 functions as an E1-like ubiquitin conjugating enzyme for the Atg12 to Atg5 and the LC3 to PE binding reaction. Before lipidation, LC3 needs to be cleaved by Atg4. Atg10 acts as an E2-like ubiquitin conjugating enzyme by transferring the Atg12/Atg5 complex to Atg16L. This newly-formed triple complex mediates the binding of the lipidated LC3 to the autophagosome membrane 


\subsubsection{Atg $8 / L C 3$}

Yeast Atg8 has at least eight orthologues in mammalians that can be divided into two subfamilies depending on their sequence homology: the microtubule associated protein 1Light $\underline{\text { Chain }} \underline{3}$ (map-LC3 or LC3) and the $\gamma^{-}$-aminobutyric acid receptor-associated protein (GABARAP)/GATE16 family. There are four LC3s, LC3A with two variants from alternative splicing events, LC3B (the best studied form) and LC 3C. Furthermore, there are the GABARAPs, GABARAP, GABARAPL1, GABARAPL2 (generally called GATE-16) and GABARAP3. All of these proteins are important for macroautophagy but have different functions. The LC3 subfamily plays a role in elongation of the isolation membrane whereas the GATE-16/GABARAPs are more important for autophagosome maturation (Weidberg et al. 2010). Atg4/autophagin (which also has various isoforms in mammals) cleaves LC3 into the cytosolic form, LC3I, which can become lipidated and bound to the autophasome membrane, then called LC3II. Apart from phagophore elongation, LC3 could also be implicated in autophagosome movement. The autophagosome has to move from the cell periphery to the perinuclear region in order to fuse with lysosomes. This happens through a mechanism that depends on microtubules (Jahreiss et al. 2008) and LC3 has a microtubule-binding motif. In vitro injection of antibodies against the region of LC3 containing this motif caused inhibition of autophagosome movement (Kimura et al. 2008) Another interesting finding for LC3 is that it binds directly to p62, also known as Sequestosome-1 (SQSTM1), a polyubiquitin-binding protein that is degraded by autophagy (Pankiv et al. 2007). Although macroautophagy is normally thought to be unspecific, p62 could recognise harmful proteins and target them for autophagic degradation. Interestingly, excessive p62, for example due to impaired autophagy, causes liver damage in mice (Komatsu et al. 2010) and probably contributes to tumourigenesis (Mathew et al. 2009). Both LC3 and p62 can be used to monitor autophagy as they undergo changes on autophagy induction which can be detected by Western blotting and immunofluorescence assays (Mizushima et al. 2010).

\subsubsection{Ubiquitin-like systems}

There are two ubiquitination-like protein conjugation systems involved in phagophore elongation (Yang \& Klionsky). These mechanisms are evolutionarily conserved from yeast to humans. Atg12 and LC3/Atg8 are ubiquitin-like modifiers. Atg7 acts as an E1 enzyme for both because it catalyses the covalent binding reactions of Atg12 to Atg5 as well as that of LC3/Atg8 to phosphatidylethanolamine (PE). In the next step, Atg10 acts as an E2 enzyme for Atg5-Atg12, and Atg3 does the same for LC3-PE (Ichimura et al. 2000). 
Atg5-Atg12 form a protein complex with homo-oligomerised Atg16. This complex could act as an E3 system for the conjugation of PE to LC3, as lipidation of LC3 happens downstream of Atg5-Atg12 conjugation and the Atg5-Atg12-Atg16 complex could function as a scaffold for this (Yoshimori \& Noda 2008). Lately the mammalian Atg16-like protein, Atg16L, has become a focus of attention because of the description of single nucleotide polymorphisms (SNPs) linked to Crohn's disease (Hampe et al. 2007). Crohn's disease is a common auto-immune medical condition in which the patients suffer from chronic inflammatory bowel disease, probably due to a defect in the regulation of the immune response. As the point mutation in $\operatorname{Atg} 16 \mathrm{~L}$ (T300A) is found within a domain absent in yeast this could be a case of an evolutionarily acquired response of autophagy to immune stimuli.

In this context it is interesting to note that there is an Atg5/Atg7-independent type of macroautophagy. This pathway depends on Rab9 (a member of the RAS oncogene family), is regulated by Ulk1 and Beclin1 and does not involve lipidation of LC3 (Nishida et al. 2009b). In this type of autophagy, autophagosomes seem to be generated by fusion of isolation membranes with vesicles from the trans-Golgi and late endosomes. On the one hand, Beclin1 deficient mice (Yue et al. 2003) die before they are born, as do knock-outs of two other proteins which form a complex with Beclin1, FIP200 (Liang et al.) and Ambra1 (Fimia et al. 2007). On the other hand, Atg5 and Atg7 KO-mice seem normal until they are born and die because they cannot survive the neonatal starvation period (Kuma et al. 2004; Komatsu et al. 2010). Presumably, the fact that there is a type of macroautophagy which does not depend on Atg5 and Atg7 makes it possible for the Atg5- and Atg7-deficient mice to survive at least until they are born.

\subsubsection{Atg-gene knockout and its implications for autophagy research in vitro}

A number of mice lacking different autophagy related genes have been generated and their use as model systems has confirmed a critical role in autophagy of a number of the mediators mentioned above. However, the knockout of LC3B (Cann et al. 2008) or Atg4C (Marino et al. 2007) leads to mild phenotypes with regard to autophagy deficiency whereas knockout or knockdown of Atg3 (Sou et al. 2008), Atg5 (Mizushima et al. 2001), Atg7 (Komatsu et al. 2005), Atg16L (Cadwell et al. 2008; Fujita et al. 2009), Ambra1 (Fimia et al. 2007), Beclin1 (Qu et al. 2003) or FIP200 (Hara et al. 2008) results in strong reduction or complete deficiency of autophagy. The reason for the mild effect of LC3B and Atg4C knockout is probably their existing isoforms. 
With regard to autophagy research in the laboratory using cultured cells, the genes LC3B and $\mathrm{Atg} 4 \mathrm{C}$ are not suitable as siRNA targets, due to the mild phenotype observed in mice. Knockdown of Atg5, Atg7 or Beclin1 seems to be the more reliable manner to investigate involvement of autophagy in vitro. Instead of gene silencing, another type of genetic approach has proven to be useful to study the function of autophagy related proteins. Thus, the use of dominant negative forms of autophagy proteins, as for example a kinase dead mutant of Ulk1 (Chan et al. 2009) has confirmed the role of this kinase in the autophagic process.

\section{Fusion events in macroautophagy}

In mammalians, autophagosomes fuse with vesicles of the early and late endosomal compartments (Tooze et al. 1990), as well as multivesicular bodies, and are subsequently called amphisomes or late autophagic vesicles (Orsi et al. 2010). These fusion events may be helpful for acquiring different factors prior to the final fusion with lysosomes. Fusion of autophagosomes with endosomes seems to depend on endosomal membrane proteins but not on lipidated LC3 or ATP (Morvan et al. 2009).

\subsection{Kiss and run encounters}

In order to fuse with lysosomes, the apparently random-forming autophagic vesicles need to move along microtubules which direct them to the centre of the cell. Here it seems that the encounters with the lysosomes do not necessarily result in complete fusion, since in normal rat kidney cells a "kiss-and-run" mechanism was the most frequent type of liaison. This predominantly resulted in a transfer of small portions of the autophagic vesicle content into the lysosome rather than vice versa. It is estimated that only about a third of the lysosome-autophagosome encounters lead to an autolysosome (Jahreiss et al. 2008).

\subsection{Molecular mechanisms in autophagosome fusion}

As in many other membrane trafficking events the small GTPases of the rab family also mediate autophagosome fusion with lysosomes and, in particular, it is known that for their complete fusion the little GTP-ase, Rab7 is needed. Consistent with this, Rab7 density on autophagosome membranes increases during the maturation process (Gutierrez et al. 2004b). 
Autophagosome maturation also seems to depend on hrs (hepatocyte growth factorregulated tyrosine kinase substrate) which plays a role in the sorting of endosomes (Tamai et al. 2007).

Another player in the autophagosome fusion with the lysosome seems to be SKD1 which is the mammalian homologue of yeast Vps4. SKD1 and Vps4 are AAA ATPases that are also involved in sorting and transport from endosomes. Expression of a dominant negative mutant of SKD1 led to a defect in autophagic degradation and, judging by the vacuolar morphology observed, this was caused by inhibited autolysosome formation (Nara et al. 2002).

The lysosome-associated-membrane-proteins LAMP1 and LAMP2 are estimated to make up about half of the lysosomal membrane proteins and seem to have common functions in vivo. Both the LAMP1 or LAMP2 knockout mice are viable although the latter are more severely affected (Tanaka et al. 2000). These mice show extensive accumulation of autophagic vesicles as a result of defective fusion with lysosomes, while fusion of the autophagosomes with endosomes does not seem to be affected. In-vitro, the accumulation of autophagic vesicles can also be observed in LAMP2-lacking hepatocytes or double knock-out LAMP2/LAMP1-deficient fibroblasts (Eskelinen 2006). The reason for impeded autophagosome-lysosome fusion in LAMP2-deficient cells is likely to be the disturbed dynein-mediated transport of the lysosomes to perinuclear regions where the lysosomes usually fuse with the autophagosome (Saftig et al. 2008). This mechanism has already been reported for other fusion events with lysosomes, such as phagosomelysosome fusions (Huynh et al. 2007).

Defects in other lysosomal proteins such as the lysosomal proteinase cathepsin D also caused autophagic vacuoles to accumulate, as has been demonstrated in 20-day old mouse brains. The reason for this accumulation, though, remains unclear (Koike et al. 2000).

Another protein with significance for autophagosome maturation is the Alzheimer's disease (AD) related protein, presenilin-1 (PS-1), a ubiquitous transmembrane protein also present in autophagic vacuoles. As part of a protein complex, one of its main functions involves regulation of intramembrane protein cleavage of different proteins including APP (amyloid beta precursor protein). Shedding of APP leads to generation of the neurotoxic amyloid beta $(A \beta)$, a peptide which is found in the $A D$ plaques in neurons. Mutant PS- 1 in mice leads to increased amounts of $A \beta$ (Citron et al. 1997). It was long believed that the pathological effect of mutant PS-1 was mainly caused by increasing $A \beta$ production. However, recent work has shown that in neurons from PS-1 hypomorphic mice, substrate 
proteolysis and autophagosome clearance during macrophagy were impaired because of decreased autolysosome acidification and cathepsin activation. It was demonstrated that acidification of autolysosomes and cathepsin activation depended on a subunit of a Iysosomal proton pump (V-ATPasVOa1), and that depleted or defective PS-1 resulted in incomplete transport of this proton pump from the ER to the lysosomes (Yamamoto et al. 1998; Lee et al. 2010). This finding could explain the decreased autophagic function in PS-1-deficient cells.

\section{The role of autophagy in disease and ageing}

Autophagy or defective autophagy has been linked to many pathologies such as neurodegeneration, cancer and infectious diseases (Mizushima et al. 2008). Due to its functions in innate and adaptive immunity, autophagy is important for the immune response. In the heart it affects the shaping of the cardiac muscle cells and contributes to cellular homeostasis. Because of its function as a stress response, the autophagic degradation pathway also plays a role in life-span extension and ageing (Cuervo 2008).

\subsection{Autophagy in neurodegeneration}

Autophagy is generally believed to have a beneficial effect preventing neurodegeneration. In neurons, autophagy is more important for normal housekeeping than for the starvation response. Aggregate-prone proteins which would harm the cells if not removed are often eliminated by autophagy, and this activity seems to be particularly relevant in the nervous system.

In the case of Huntington's disease (HD), a poly-glutamin mutation at the N-terminal region of the huntingtin protein ( $h \mathrm{tt}$ ) causes impaired clearing of the protein which then forms toxic oligomers leading to neuronal cell death (Davies et al. 1997; Ravikumar et al. 2002). This leads to impaired muscle coordination and dementia in the affected person (Andrew et al. 1993). Compromised macroautophagy is thought to be the cause of insufficient htt degradation probably due to a cargo recognition failure of cytosolic components (Martinez-Vicente et al. 2010).

Another neurodegenerative disorder that has been linked to impaired degradation by autophagy is Parkinson's disease (PD). Patients suffering from this illness show symptoms such as stiffness and tremor due to insufficient formation and action of dopamine. The loss of dopaminergic neurons is accompanied by protein accumulations inside the cells (called Lewy bodies) which consist to a large extent of alpha-synuclein bound to ubiquitin. Under physiological conditions alpha-synuclein is degraded by CMA. 
However, mutant alpha-synuclein blocked the CMA receptor, not only inhibiting its own degradation but also the turnover of other proteins by CMA. At the same time, macroautophagy was up-regulated, although it could not compensate for reduced degradation under these conditions (Cuervo et al. 2004).

Lately, Alzheimer's disease (AD) has also been associated with defects in autophagy. AD is the most common form of dementia and is typically diagnosed in elderly persons over 65 years of age. In the affected brains, plaques which contain insoluble amyloid beta (A $\beta$ ) peptide accumulate, thus leading to neuronal death by apoptosis (Yankner et al. 1990). Before plaques can be detected, autophagosome-like structures accumulate in dystrophic neurites. Whether autophagy contributes to neuronal cell death in $A D$ or is a mechanism to prevent it is still under discussion. However, it seems that protein degradation and therefore degradation of the toxic $A \beta$ in these late autophagic vacuoles is impaired, $a$ blockade which, in turn, leads to a retarded autophagic flux. Interestingly, these autophagic vacuoles are sites of beta-amyloid generation as they contain the amyloid precursor protein (APP) together with preselinin-1 (PS-1) (see also section 6.) and a PSdependent $\gamma$-secretase activity, all of which form part of the proteolytic complex involved in APP processing (Yu et al. 2005).

\subsection{Autophagy in the heart}

Apart from its function in neurons, autophagy is also important in cardiomyocytes. A cardiac-specific deficiency of Atg5 induces hypertrophy, left ventricular dilatation and contractile dysfunction. These features are accompanied by extraordinarily high amounts of apoptotic cells (Nishida et al. 2009a). In the heart, autophagy is also needed to maintain cardiomyocyte size along with global cardiac structure and function.

\subsection{Autophagy and ageing}

Autophagic activity decreases in older organisms. Together with a failure of quality control mechanisms in post-mitotic cells, reduced lysosomal degradation causes an increase in damaged proteins and dysfunctional mitochondria that are toxic for the cell.

Therefore, as a catabolic pathway, autophagy could explain why caloric restriction leads to lifespan extension, a well established finding that has been extensively studied in $C$. elegans. Animals fed on a low-calorie diet have fewer damaged components within their cells than animals of the same age fed ad-libitum (unlimited access to feed) (Hansen et al. 2008). Indeed, macroautophagy activation is a common feature of long-lived mutant worms, while defective autophagy decreases lifespan (Toth et al. 2008). Therefore less 
effective macroautophagy and chaperone-mediated autophagy cause a decline in proteinand organelle-turnover at a higher age. In the case of CMA, lowered efficiency seems to be due to fewer CMA receptors in the lysosome membrane (Cuervo et al. 2004). In macroautophagy, apart from changes in susceptibility to hormonal regulation by insulin in older organisms, newly formed autophagosomes take longer to fuse with lysosomes and degrade the cargo (Terman 1995). The latter event shows similar features to the formation of autophagosome-like structures observed in early Alzheimer's disease, a latein-life-onset disease. Age is not only a risk factor for certain neurodegenerative diseases but also for most forms of cancer, and autophagy is thought to function as a tumour suppressor as it removes damaged organelles and contributes to chromosome stability (Levine \& Kroemer 2008).

\section{Autophagy and immunity}

It has long been known that protein degradation is involved in immunity. While the function of the main selective degradation system in the cell, the proteosome-ubiquitin system, has been extensively studied in immunity (Bhoj \& Chen 2009), the role of autophagy in this context has only started to become unveiled. Thus, over the last few years numerous studies have brought to light a crucial involvement for autophagy in both innate and adaptive immunity.

For example, in a macroautophagy-like process which sometimes is referred to as xenophagy (Levine 2005), pathogens or their products are sequestered and degraded in autolysosomes. This breakdown of pathogens has been linked to presentation of the derived antigens on MHC class II, thus disclosing the dual function of autophagy in innate and adaptive immune response. In addition, autophagy is regulated by immunomodulatory cytokines such as IFN $\gamma$, TNF- $\alpha, \mathrm{IL}-4$, and IL-13 (Deretic 2009). The pattern recognition receptors (PRRs) also regulate autophagy, and autophagy seems to facilitate access of microbial molecular patterns to PRRs. Another function of autophagy in adaptive immunity seems to be the regulation of T and B-cell homeostasis (Miller et al. 2008; Nedjic et al. 2008).

\subsection{Targeting of microbes to autophagosomes (xenophagy)}

A few years ago the cytosolic group A Streptococcus was shown to be degraded in nonphagocytic cells by a process which relies on the autophagy protein $\operatorname{Atg} 5$ and takes place in vacuoles that are reminiscent of autophagosomes (Nakagawa et al. 2004). 
Since this study was published, several articles have linked ubiquination of cytosolic bacteria to degradation by autophagy. As mentioned in section 5.3.1., ubiquinated cargoes are thought to be recognised by adaptor proteins such as p62. These molecules can bind to LC3 and ubiquitin simultaneously and thereby mark the ubiquinated proteins for autophagic degradation. An example of this targeting of bacteria to autophagosomes is Salmonella enterica serovar ( $S$. typhimurium). This pathogen is normally found in intracellular vacuoles called Salmonella containing vacuoles (SCV), but as soon as the bacteria escape the SCV to invade the cytosol they are coated with ubiquitin and degraded by autophagy which keeps the cytosolic $S$. typhimurium population low. Consistently, Atg5-/- (and therefore autophagy-deficient) cells showed higher intracellular growth of $S$. typhimurium than wild type cells (Birmingham et al. 2006).

Therefore, it seems that efficient bacteria replication in the cytosol is inhibited by autophagy and thus it is not surprising that there are examples of intracellular pathogens that have developed methods to circumvent being recognised by the autophagic machinery. An example of this is Listeria monocytogenes. These bacteria express the surface protein, ActA, which is important for bacterial motility and can bind to host proteins implicated in actin nucleation. In a recent study, L. monocytogenes expressing defective ActA unable to bind to host cell proteins, were shown to undergo autophagy in an ubiquitination and recruitment of p62 involving process. However, targeting of the mutant bacteria to autophagosomes did not seem to be caused by the loss of actin-based motility. The explanation given by the authors for the importance of ActA in avoiding autophagic degradation is that by binding host cell molecules, L. monocytogenes appears to be part of the invaded cell and thereby hides from autophagy as a defence mechanism (Yoshikawa et al. 2009). Listeria can also inhibit autophagosome maturation with its toxin, Listeriolysin $\mathrm{O}$, which forms pores in the lysosomal membrane (Meyer-Morse et al. 2010).

Another bacterium which seems able to escape autophagy is Shigella flexnieri. The Shigella VirG protein is necessary for intracellular motility and binds to Atg5. Through the contact with Atg5, an autophagic process leading to the elimination of the invader is induced. Yet, the bacterium has developed a mechanism to avoid the binding of VirG to Atg 5 by secreting a protein (IscB) able to inhibit Atg5 interaction with VirG competitively (Ogawa et al. 2005).

Bacteria are not the only organisms attacked by autophagy. In mouse brains, autophagy protects against infection with neurotrophic alphavirus Sindbis in an Atg5- and p62dependent manner by targeting the viral capsid to autophagosomes (Orvedahl et al. 
2010). Another neurotrophic alphavirus, the Herpes simplex virus type 1 (HSV-1), also seems to be degraded by xenophagy (Talloczy et al. 2006). In fact, as stated in section 5.2., the HSV-1 has developed a countermeasure against autophagy by expressing the gene product ICP 34.5 that binds and inhibits Beclin1, thereby impeding membrane genesis during macroautophagy (Orvedahl et al. 2007).

Some protozoans are also removed by autophagy as is the case with Toxoplasma gondii. This organism can be trapped in autophagosome-like structures that eventually fuse with lysosomes for degradation (Ling et al. 2006).

\subsection{Pattern recognition receptors}

Pattern recognition receptors (PRR) respond to non-self molecules derived from viruses or bacteria, a cohort of components that have been called pathogen associated molecular patterns (PAMP). Examples of non-self molecules recognised by the PRRs are the bacterial cell wall constituents, peptidoglycan (PGN) and lipopolysaccharide (LPS), or nucleic acids such as viral DNA and RNA (Delbridge \& O'Riordan 2007; Deretic 2009). The PRRs consist of four major classes; the nucleotide-binding-and-oligomerizationdomain (NOD)-like receptors (NLRs), the toll-like receptors (TLRs), the retinoic acidinducible gene I ( $\left.{ }_{-} \mid \mathrm{IG}-\mathrm{I}\right)$-like helicases (RLHs), and the C-type lectin receptors.

\subsubsection{Nod-like receptors}

Up till now, the mammalian Nod-like receptor family has been found to include 21 members. All of these members contain a common domain called NACHT domain needed for self-oligomerisation and nucleotide binding. Additionally, most NLRs also contain a leucine rich repeat (LRR) domain that mediates the binding to PAMPs (Lamkanfi \& Dixit 2009). The NLR proteins IPAF (ICE protease-activating factor), the NALPs (NACHT domain-, leucine-rich repeat-, and PYD-containing proteins), and NAIP (neuronal apoptosis inhibitory protein) form the inflammosomes. These are multiprotein complexes which lead to maturation of the proinflammatory cytokines, and thus promote innate immunity. There appear to be different inflammosomes depending on the type of PAMP that acts as an inducer (Martinon et al. 2007).

Apart from the inflammosome NLR subgroups, (the NALPS and IPAF/NAIP) there are the Nods, Nod 1 and Nod 2, which can recognise bacterial peptidoglycan (PGN) or its breakdown products and lead to the activation of the transcription factor NFKB via the kinase RIP2 (Shaw et al. 2010). In a recent study, viral single stranded RNA was shown to induce production of antiviral IFN- $\beta$ through activation of Nod2 but not RIP2, suggesting 
alternative Nod2 signalling mechanisms (Sabbah et al. 2009). As Nod1 is expressed in epithelial cells and fibroblasts and Nod 2 is found in myeloid cells and Paneth cells, these NRL have a broad expression pattern. Both Nod1 and Nod2 have been shown to induce autophagy in vitro and in vivo upon stimulation with PGN, although this happens in a RIP2 and NFKB independent manner. Moreover, they seem to recruit Atg16L to the plasma membrane where the bacteria enter the cell. Here the invader is enwrapped in an autophagosome for degradation and elimination (Travassos et al. 2010). Interestingly, polymorphisms in both genes, ATG16L and Nod2, have been related to a higher risk for developing Crohn's disease, an autoimmune pathology previously mentioned (see 5.3.2.). Taken together, these insights imply affected Nod2-dependent autophagy as a causal event in Crohn's disease (Sumpter \& Levine 2010).

\subsubsection{Toll-like receptors}

TLRs are type 1 membrane proteins located in topologically external spaces such as the plasma membrane or the endoplasmatic reticulum of macrophages, plasmacytoid dentritic cells and other immune cells (Medzhitov 2001). TLRs lead to production of proinflammatory cytokines and type I interferon via adaptor molecules such as MyD88 (the

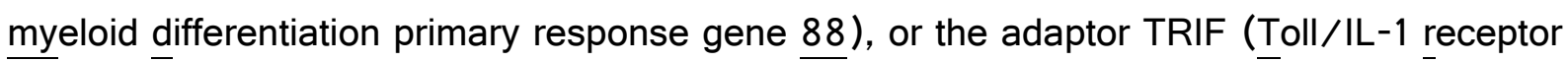
domain-containing adaptor inducing IFN- $\beta$ ) (Saitoh \& Akira 2010). TLRs also regulate phagocytosis of bacteria (Blander \& Medzhitov 2004) and can recognise PAMPs of viral, eukaryotic or prokaryotic origin. TLR7 and TLR8 were shown to recognise single stranded RNA, while double stranded DNA has been found to be a ligand for TLR9 (Diebold et al. 2004; Heil et al. 2004; Krug et al. 2004). The yeast PAMP zymosan and bacterial cell wall constituent peptidoglycan induce TLR2, while TLR4 can be stimulated by LPS (lipopolysaccharide) from the cell wall of gram-negative bacteria (Takeuchi \& Akira 2001).

\subsubsection{Autophagy upstream of TLR-signalling}

One of the various tasks that autophagy fulfils in innate immunity seems to be the transportation and presentation of the PAMP ligands to appropriate TLRs as has been shown in plasmacytoid dendritic cells (pDCs). pDCs are a subset of the antigen presenting dendritic cells which rely fully on TLR7 and TLR9, and interact with B- and Tcells once activated. pDCs are thought to be able to respond to infections without being themselves infected as the TLRs are located on the cell surface and endosomal compartments, which topologically means that the receptor-ligand interaction happens 
outside the cell. Therefore, the contact of the pathogen-derived ligand with the TLR from the pDC can take place before the pathogen actually enters the cell (Takeuchi \& Akira 2007). An interesting study demonstrated that recognition of the viral TLR7 ligand (single stranded RNA (ssRNA)), and therefore subsequent type I IFN production as a response to infection, depended on Atg5. The authors explained this by the fact that the cytosolic viral sSRNA had to be transported to endosomal-lysosomal compartments by autophagy in order interact with TLR7 (Lee et al. 2007a).

\subsubsection{Autophagy as a consequence of TLR signalling and its role in microbial phagocytosis}

Autophagy not only helps to present antigens to TLRs but can also be induced on contact of TLRs with pathogens. In RAW macrophages, TLR7 has been shown to induce autophagy after activation with imiquimod, an artificial TLR7 agonist. Autophagy induction depended on MyD88 as well as on Beclin1, and was able to improve elimination of intracellular microbes. In this study pathogen-induced autophagy was linked to TLR signalling (Delgado et al. 2008). Another study found the same type of link. Here, in murine macrophages, stimulation of TLR4 with LPS induced macroautophagy through a TRIF-dependent (but MyD88-independent) pathway, and thereby enhanced elimination of mycobacteria. Receptor-interacting protein1 (RIP1) and p38 mitogen-activated protein kinase (MAPK) are downstream components of the TLR4/TRIF pathway and were demonstrated to be involved in the signalling cascade that led to autophagy in this context (Xu et al. 2007).

As mentioned above, TLR-signalling is implicated in phagocytosis of microbes. Phagocytosis is a process mainly carried out by specialised macrophages and in which microbes are engulfed from extracellular sources to form a phagosome inside the cell. Phagosomes undergo a subsequent maturation process to eventually fuse with lysosomes where the content is degraded. In this context, autophagy has also been proposed as being an additional removal mechanism of pathogens in phagosomes. An example is provided by $M$. tuberculosis containing phagosomes which degrade the enclosed bacteria through an autophagy-dependent mechanism (Gutierrez et al. 2004a).

In another interesting study, TLR activation was shown to be a link between autophagy and phagocytosis since TLR-signalling enhances the microbicidal properties of phagosomes (Sanjuan et al. 2007). In this article it is shown that latex beads coated with TLR ligands and fed to macrophages for phagocytosis induce Atg5/Atg7 dependent localisation of LC3 to the phagosomes. Inspite of Atg5, Atg7 and LC3 forming part of the 
typical macroautophagy machinery which leads to formation of the characteristic double membrane, only single membranes could be observed in these LC3-positive phagosomes. Although in all these studies autophagic activity was measured by LC3II levels and localisation of GFP-LC3 in autophagosomes, the results did not always show the features of typical autophagy, as for example in the last cited study by Sanjuan et al., where the GFP-LC3 vacuoles had only a single membrane (Sanjuan et al. 2007). To what extent the Atg-dependent processes observed upon TLR stimulation constitute canonical macroautophagy, or how alike to macroautophagy they are, remains unclear.

\subsubsection{Retinoic acid-inducible gene / (RIG-I)-like helicases}

The retinoic acid-inducible gene I (RIG-I)-like helicases (RLHs) are present in the cytosol of the cell and mainly recognise nucleic acids from viral pathogens. Just like the TLR, the $\mathrm{RLH}$ lead to expression of type 1 interferons (IFN), especially interferon alpha, and other proinflammatory cytokines (Taniguchi \& Takaoka 2002). However, as the RLHs are only present in the cytosol, the potent antiviral cytokines cannot be induced prior to infection, and therefore systemic infections are mainly responded to by TLRs in the pDCs.

\subsection{Induction of autophagy by cytokines}

Cytokines regulate autophagy positively and negatively. For example, proinflammatory IFN $\gamma$ and TNFa induce autophagy, while anti-inflammatory IL-4 and IL-13 inhibit it. Although TNFa has been used as an autophagy agonist, the mechanism by which it does so remains unclear (Deretic 2009). As there seems to be crosstalk between apoptosis and autophagy pathways (see 10.3.), and TNFa can activate apoptotic cell death (see 10.1.1.), the autophagy-inducing properties of TNFa could be related to this autophagyapoptosis interplay. This was implied by a study in which activation of the anti-apoptotic transcription factor NFKB, (a downstream target of TNFa) repressed autophagy induction by TNFa (Djavaheri-Mergny et al. 2006).

How IFN $\gamma$ induces autophagy in humans also remains unclear. Studies in mice showed that IFN $\gamma$ induces autophagy through an immunity-regulated GTPase (IRG). However, whilst in mice there are many members of this family that have been discovered because of their role in cell-autonomous defence against pathogens, in humans, only three IGRs have been identified. Also, in mice, IFN $\gamma$ further leads to expression of IGR via the transcription factor STAT, whereas the only immunologically relevant IRG paralog in humans, IRGM, is constitutively expressed. Nevertheless, it seems that human IGRM is 
necessary for full activation of autophagy upon induction by IFN $\gamma$, starvation or rapamycin (Singh et al. 2006).

The manner in which the anti-autophagic cytokines inhibit autophagy seems clearer. IL-4 and IL-13 function via the Akt signalling pathway and Akt lies upstream of mTOR (see figure 2) which is a major inhibitor of autophagy.

Activation of autophagy by cytokines and its role as a defence mechanism against intracellular microbes could account for a phenomenon called Th1/Th2 polarisation. This describes the fact that $\underline{T}$-helper cells type $\underline{1}$ (Th1) and T-helper cells type $\underline{2}$ (Th2) have contrary effects in protecting against intracellular pathogens and in macroautophagy. A possible mechanistic explanation is that Th1 produce IFN $\gamma$ and TNF- $\alpha$, both of which induce autophagy, but Th2 cytokines IL-4 and IL-13 inhibit autophagy (Deretic 2010).

An example of an intracellular pathogen which Th1 cytokines combat but the survival of which is favoured by Th2 cytokines is Mycobacterium tuberculosis. The bacterium can persist in the human population as a latent infection thanks to its macrophage parasitism. M. tuberculosis impedes phagosome maturation enabling the microbe to persist in these phagosomes and avoid disposal. IFN $\gamma$-induced autophagy in macrophages led to localisation of autophagical markers on the phagosomes containing mycobacteria and by this means caused increased death of the pathogen (Gutierrez et al. 2004a). However, Th2 cytokines IL-4 and IL-13 abrogated autophagy and improved the survival of mycobacteria in macrophages. Therefore autophagy seems to be the effector of the Th1/Th2 polarisation at least in this context (Harris et al. 2007).

\subsection{Adaptive immunity}

Apart from the Th1/Th2 polarisation, autophagy is also involved in other aspects of adaptive immunity and autoimmunity. In professional antigen presenting cells (APC), extracellular antigens are internalized and processed within the lysosomal compartments before they bind major histocompatibility $(\mathrm{MHC})$ class II molecules and are presented to $\mathrm{T}$ cells. The LAMP-2A-hsc70 complex which mediates CMA plays an important role in this process of immunological recognition and antigen presentation (Zhou et al. 2005). Macroautophagy degradation products are also presented to $\mathrm{CD} 4^{(+)} \mathrm{T}$-cells by $\mathrm{MHC}$ class II (Gannage \& Munz 2009) and, at least in macrophages infected with HSV-1, autophagy facilitated presentation of viral antigens on MHC class I (English et al. 2009).

Autophagy also averts autoimmunity by shaping the CD4 T-cell repertoire through the recognition of self ligands bound to $\mathrm{MHCll}$ on thymic epithelial cells (TECs). It is crucial that $\mathrm{T}$-cells become tolerant of self antigens to avoid autoimmune reactions such as 
severe colitis and multi-organ inflammation. Autophagy delivers MHC-II-peptides from the intracellular milieu of TECs and thereby assists in self-antigen presentation and T-cell selection (Nedjic et al. 2008). Another task for autophagy related to T-cells is ensuring their survival and proliferation. Apart from inhibiting apoptosis in this cell type, autophagy seems to be necessary for efficient proliferation following T-cell receptor stimulation and could regulate T-cell homeostasis (Pua \& He 2007).

\section{Autophagy in tumourigenesis}

As previously stated, autophagy seems to work as a tumour suppressor mechanism, at least at an early stage of tumourigenesis. Consistent with this idea, Beclin1, part of a protein complex implicated in phagophore elongation (see 5.2.), is a tumour suppressor monoallelically deleted in $40-70 \%$ of sporadic breast or ovarian cancers (Liang et al. 1999). In addition, two groups have shown that haplodeficient Beclin1 mice develop spontaneous cancers such as lymphoma, lung cancer and hepatocellular carcinoma (Qu et al. 2003; Yue et al. 2003). Other members of the Beclin1/Vps34 protein complex also seem to be tumour suppressors. For instance, the gene encoding for UVRAG is often monoallelically deleted in colon cancers (Liang et al. 2006). The fact that oncogenes such as Akt and Bcl-2 (see section 5) inhibit autophagy, while autophagy inducers including Atg4C, Beclin1, UVRAG, Bif-1 and PTEN (for more information on the mentioned proteins see section 5) are oncosuppressors, strongly implies that autophagy has a beneficial effect on protection against tumours (Morselli et al. 2009). There are different hypotheses as to why defective autophagy promotes tumourigenesis. One reason could be that autophagy inhibition favours necrotic cell death and inflammation which might benefit tumour growth (Degenhardt et al. 2006). Another possibility is that deficient autophagy leads to accumulation of old and damaged organelles or proteins which are possible sources of genotoxic compounds such as reactive oxygen species (ROS) (Lemasters 2005). These chemical species can damage DNA and could account for the finding that compromised autophagy favours chromosomal instability (Mathew et al. 2007).

\section{1. $p 53$ and its effectors}

The most commonly mutated tumour suppressor, p53, has a dual role in autophagy regulation as it can induce or inhibit autophagy. Initially p53 was known, above all, as a transcription factor in the nucleus, which activated transcription of genes involved in apoptosis or cell cycle arrest upon DNA damage (Vogelstein et al. 2000). Later, cytosolic 
p53 was found to induce apoptosis (see section 10.1. for more information on apoptosis) through a direct activation of the pro-apoptotic modulator Bax, thereby leading to mitochondrial membrane permeabilisation and setting off this type of programmed cell death (Chipuk et al. 2004). However, cytosolic as well as nuclear p53 also control autophagy. Target genes of the transcription factor p53 include negative regulators of mTORC1, a complex that regulates autophagy negatively. Thus, p53-induced transcription of AMPK and PTEN (which inhibit mTORC1, for further details see figure 2 ) leads to autophagy. Sestrin1 and Sestrin2 have also been identified as p53 gene targets, and their products induce AMPK (Budanov \& Karin 2008). A Sestrin2-mediated stimulation of autophagy via mTOR was described recently as a response to genotoxic stress (Maiuri et al. 2009). p53 can further induce the damage-regulated autophagy modulator (DRAM), an autophagy inducer that also plays a role in p53-dependent apoptosis. In tumours one can find decreased expression of this protein which is essential for p53 dependent autophagy and apoptosis (Crighton et al. 2006).

However, whereas nuclear p53 induces autophagy through transcriptional events, cytosolic p53 seems to inhibit autophagy by a transcription-independent mechanism, thus implying that disappearance of p53 from the cytosol can induce autophagy. The latter was shown by depletion of p53 in colon cancer cells as well as in mouse embryonic fibroblasts which resulted in subsequent vacuolisation of GFP-LC3 that depended on Atg5, Atg6/Beclin1 and other autophagy related genes (Tasdemir et al. 2008).

Considering that in the great majority of cancers, the p53 signalling pathway is defective (Polager \& Ginsberg 2009), and that this extremely important tumour suppressor can regulate autophagy positively or negatively, it seems plausible that autophagy itself may also have different roles in tumourigenesis depending on the setting.

\subsection{Once the tumour has developed}

Whilst most data point to the notion that autophagy inhibits tumourigenesis, it is likely that macroautophagy can also help cells in solid tumours to survive limited nutrient supply, metabolic stress or chemotherapy and make them more resistant to treatment (Amaravadi et al. 2007). Whether autophagy can kill tumour cells with impaired apoptosis is still under discussion, as autophagic vacuoles in dying cells can signify both: cell death caused by autophagy or autophagy as an attempt to rescue the dying cell. 


\section{Autophagy in programmed cell death}

Different types of genetically regulated cell death are known to exist. Apoptosis is the best studied type of programmed cell death and is also referred to as programmed cell death (PCD) type I. Apoptosis involves activation of a particular protease family, the caspases, and also chromatin degradation. Correspondingly, PCD type II is an autophagic cell death which is accompanied by large scale vacuolisation of the cytoplasm. Necrosis which is characterised by organelle swelling and plasma membrane rupture has long been thought to be a non-programmed, accidental type of death, but there is emerging evidence that necrosis might be finely regulated (Kroemer \& Levine 2008).

An overview of these three types of cell death is also provided in figure 4 .

\subsection{Apoptosis}

Translated, the word apoptosis means "leaves falling from a tree" in ancient Greek. Kerr and colleagues were the first to describe two different types of cell death, apoptosis and necrosis (Kerr et al. 1972). In contrast to necrosis, apoptotic cells and their nuclei shrink while plasma membrane integrity persists much longer. As apoptosis proceeds, the plasma membrane also undergoes morphological changes and forms little blebs. This process is referred to as "blebbing". Subcellular compartments are broken down due to cleavage of cytoskeletal proteins (Martin \& Green 1995). Finally, it comes to chromatin condensation and DNA fragmentation, the resulting pieces of which can be detected as the typical 200bp-DNA ladder (Cohen et al. 1994). At the end of the process the cells are removed by phagocytosis, either by macrophages or neighbouring cells (Savill \& Fadok 2000).

Apoptosis plays a vital role in development and cell homeostasis (Meier et al. 2000), and is also involved in elimination of potentially dangerous cells. Because of its relevance in physiology, alterations in apoptosis can lead to pathologies, such as autoimmune diseases, cardiovascular and neurodegenerative illnesses, as well as cancer. Both reduced and exaggerated apoptosis can give way to pathologies. For example, while in tumours, apoptosis is often down-regulated and the malignant cells are not killed, in the neurodegenerative Alzheimer's disease there is an excess of apoptosis in the neurons (Thompson 1995). 


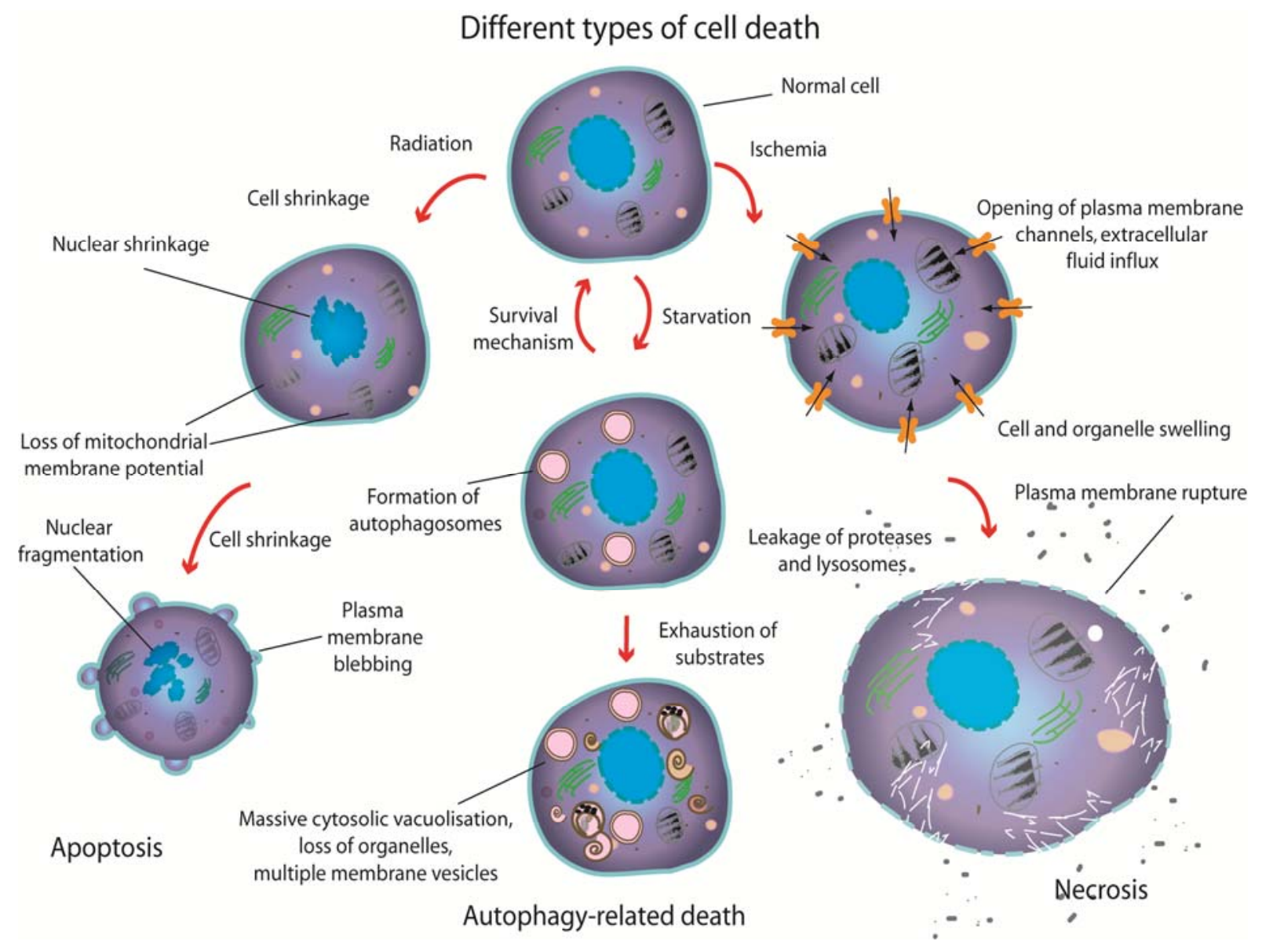

Figure 4: Apoptosis, Autophagy-related cell death and Necrosis are the three major pathways of cell death (modified from Hotchkiss et al. 2009).

Crosstalk which can occur between them is not represented in the figure. Apoptosis and autophagy-related cell death are programmed types of cell death while necrosis is believed to be accidental although there has been evidence found which suggests that at least some types of necrotic cell death are programmed. Which type of cell demise occurs depends, amongst other criteria, on the stimulus and cell type.

\subsubsection{The two apoptosis pathways (extrinsic and intrinsic)}

There are two main pathways in apoptosis and both lead to activation of caspases, the principal apoptosis executioners (Martin \& Green 1995). On the one hand, there is the extrinsic or death-receptor pathway that is activated by members of the TNF (tumour necrosis factor) superfamily which bind to death receptors in the plasma membrane. This leads to formation of a multiprotein death-inducing signalling complex (DISC) and activation of caspase 8 (Nagata 1997). On the other hand, there is the intrinsic pathway that proceeds via the mitochondria. Intracellular insults such as a high concentration of ROS, radiation and DNA damage, growth factor deprivation or the unfolded protein response lead to increased mitochondrial permeability and release of proapoptotic proteins (in particular cytochrome $\mathrm{C}$ and SMAC/DIABLO) from the mitochondrial intermembrane space. Cytochrome $C$ participates in caspase 9 activation and SMAC/DIABLO 
antagonises caspase inhibitors. The mitochondrial membrane permeabilisation is tightly regulated by the $\mathrm{Bcl}-2$ protein family and the interplay of its pro- and anti-apoptotic members. Anti-apoptotic $\mathrm{Bcl}-2$ was the first protein discovered from this group. Family members of the $\mathrm{Bcl}-2$ family have at least one $\mathrm{Bcl}-2$ homology $(\mathrm{BH})$ region which enables them to interact with other family members. Three subfamilies can be differentiated according to functional and structural criteria. First, there are the pro-survival proteins $\mathrm{Bcl}-2, \mathrm{Bcl}-\mathrm{xl}, \mathrm{Bcl}-\mathrm{w}, \mathrm{Mcl}-1, \mathrm{~A} 1$ and Boo/Diva that have multiple (the majority of them have four) $\mathrm{BH}$ regions. The second group, the pro-apoptotic members $\mathrm{Bcl}-2-$ associated $\underline{X}$-protein (Bax) and the $\underline{B} \mathrm{cl}-2$-homologous-antagonist-killer (Bak) have three $\mathrm{BH}$ regions (Oltvai et al. 1993; Chittenden et al. 1995; Kiefer et al. 1995). Bak is found in the membranes of the mitochondria and the endoplasmic reticulum, whereas Bax is usually cytosolic but localises to these membranes once it has been activated.

The third subfamily includes the $\mathrm{BH}$-only proteins with only one $\mathrm{BH}$ domain through which they bind the anti-apoptotic Bcl-2s and thereby liberate the proapoptotic Bak and Bax proteins. Examples for $\mathrm{BH} 3$-only proteins are Puma (p53-upregulated modulator of apoptosis), Bid and Bim (Wang et al. 1996; O'Connor et al. 1998; Nakano \& Vousden 2001; Yu et al. 2001).

Caspase 8 from the death receptor pathway as well as caspase 9 from the mitochondrial pathway activate the executing caspases 3,6 and 7 which lead to massive cleavage of proteins and finally the death of the cell (Danial \& Korsmeyer 2004). Caspase 3 and caspase 7 are probably the most important in consideration of the fact that caspase 3-or caspase 7-deficient mice are defective in apoptosis and die immediately after birth (Lakhani et al. 2006). Whether the intrinsic or extrinsic pathway is activated depends on the type of the apoptotic stimulus, and in certain situations, it is possible that both pathways are activated at the same time (Hotchkiss et al. 2009).

\subsection{Autophagic cell death}

Although it is controversial whether autophagic cell death is really induced by autophagy, or whether this type of programmed cell death is only accompanied by this process, several examples indicate that a death modality truly executed by autophagy exists. For example, embryonic fibroblasts from double knockout Bak and Bax mice underwent a nonapoptotic type of death. After treatment with the apoptosis inducer etoposide, the cells became rounded, detached from the tissue culture plate and adapted a morphology that resembled a balloon. Concomitantly, the cells became permeable to propidium iodide, a staining that proves the occurrence of cell death. At the same time, autophagosomes were 
detected by electron microscopy. This type of death could not be inhibited by caspase inhibitors but by the inhibitor of autophagy, 3-methyladenine, and to some extent by knocking down the autophagic mediators Atg5 and Beclin1 (Shimizu et al. 2004). Another example of autophagic cell death under the circumstances of apoptosis suppression are mouse fibroblasts in which caspase- 8 inhibition led to Atg7- and Beclin1-dependent cell death (Yu et al. 2004). Oncogenic RAS that can work as tumour promoter by leading to increased cell proliferation has recently been shown to induce autophagic cell death. Upon oncogenic RAS induction, expression of Noxa, a $\mathrm{BH} 3$-only protein implicated in apoptosis induction (although compared to its other $\mathrm{BH} 3$-only family members, it is a rather weak inducer of apoptosis (Kuwana et al. 2005)) and expression of Beclin1 were up-regulated. In turn, this led to a type of cell death that showed various features of autophagy and was inhibitable by knockdown of Beclin1 and Atg5 (Elgendy et al. 2011). There are also "in vivo" examples of autophagy-gene dependent death such as in C. elegans, where necrotic cell death depended on autophagy and also in Drosophila larvae where salivary gland degradation is inhibited by Atg-mutants (Berry \& Baehrecke 2007; Samara et al. 2008). In addition, the fact that a Beclin1 mutant which cannot bind to its inhibitor $\mathrm{Bcl}-2$ induces not only more autophagy than wild type Beclin1 but also increased cell death implies that too much autophagy can kill the cell (Pattingre et al. 2005).

Members of the Bcl-2 family play relevant roles in both autophagy and apoptosis. There are various cases of cell death with autophagic features that are associated with $\mathrm{Bcl}-2$ family members. For example, ectopic expression of the $\mathrm{BH}$-only protein Bik, which usually induces apoptosis, led to non-apoptotic cell death in $\mathrm{Bcl}-2$ knock-out cells (Rashmi et al. 2008). In addition, the Bcl-2 interacting proteins BNIP3 and its homologue BNIP3-like (BNIP3L) are $\mathrm{BH} 3$-only proteins which are involved in hypoxia-induced cell death. Thus, expression of both proteins is positively regulated by the transcription factors FoxO3 and Hif (hypoxia induced factor). Although BNIP3L is an agonist of apoptosis, Kanzawa and colleagues (Kanzawa et al. 2005) observed a type of caspase-independent death induced by arsenic trioxide and accompanied by LC3 activation that depended on BNIP3 and BNIP3L. Because of the activation of LC3, they used the term autophagic cell death to describe the event. While autophagic cell death has been termed type $\underline{2}$ programmed cell death (PCDII), as opposed to apoptosis being type 1 programmed cell death, there is growing evidence that, at least on some occasions, necrosis is also programmed. Intriguingly, Atgs have been shown to be important for necrotic cell death (Samara et al. 2008). As the characteristics for PCDII in mammals remain to be fully defined, it cannot be excluded that in reports on non-apoptotic programmed cell death, 
necroptosis (programmed necrosis) or autophagic cell death are mistaken for each other. Both types of cell death share characteristics such as Atg-dependence and caspaseindependence. On some occasions it is even possible that the two terms are used to describe the same phenomenon. This is implied by reports that necrosis and autophagic cell death can be induced by the same protein and show similar features. For example, before linking BNIP3 to autophagic cell death, it was described as inducing a necrosis-like death. However, this necrosis-like death was accompanied by increased formation of autophagosomes and, in general, the features described for this phenomenon seem consistent with what was reported five years later as autophagic cell death (Vande Velde et al. 2000; Kanzawa et al. 2005).

\subsection{Does autophagic cell death exist?}

As previously stated, inhibition of one cell death pathway can lead to induction of another type of cell death. Thus, it has been proven that cells lacking central proteins of the apoptotic pathway such as Bak and Bax die in response to etopside, although not through apoptosis (Shimizu et al. 2004).

Similarily, in cancer cell lines, inhibition of caspases has even been shown to be sufficient to induce a type of cell death dependent on Atg7 and Beclin1 ( $Y u$ et al. 2004). Autophagy working as a killing mechanism in cancer cells has also been suggested as an explanation for the case of oncogenic H-RAS inducing cell death, since this process was inhibitable by Beclin1 and Atg5 knockdown (Elgendy et al. 2011). As an additional example of the fact that autophagy could also be an alternative mechanism to execute cell death in cells with incapacitated apoptosis, it has been reported that therapy-induced cell death with autophagic features was enhanced after apoptosis inhibition. This was the case in a clonogenic survival study with lung cancer cells and the caspase 3 inhibitor M867. These cells with blocked apoptosis were more susceptible to non-apoptotic cell death caused by radiation than without the caspase inhibitor (Kim et al. 2006).

However, Bak and Bax-deficient cells have been shown to resist cell death induced by UV radiation or serum withdrawal and the study shows that inhibition of apoptosis can lead to fewer dead cells in total, and not only fewer cells dying of apoptosis (Wei et al. 2001). Thus, whether autophagy on its own is the executioner of a programmed type of cell death is not quite as clear. The observation of autophagy in a dying cell could simply mean that the inhibition of one cell death mode led to higher levels of autophagy, which participated in the killing of an already doomed cell rather than causing, on the bottom line, a higher number of dead cells. The fact that the majority of the studies on autophagic cell death 
have been carried out in cancer cell lines or apoptosis-defective cell lines could mean that autophagy is a failsafe mechanism for killing abnormal cells that are defective in the standard death programme apoptosis.

While there are many examples of excessive autophagy in dying cells or of Atgs being involved as accomplices in killing the cell (Samara et al. 2008) (see chapter 10.2), there is no strong evidence for autophagy-executed cell death. In the example of the etoposideinduced cell death in Bak/Bax-deficient MEFs, knocking out Atg5 or Beclin1 rescued hardly $10 \%$ of the cells from dying compared to the control cells (Shimizu et al. 2004). In a more recent study, the percentage of cells rescued by Atg-knockdown rose to $50 \%$ or even more for Beclin1 silencing (Elgendy et al. 2011), thereby proving the important role of autophagy-related genes in the death process.

However, in contrast to apoptosis as a killing mechanism, in most cases autophagy does not seem to provoke cell death. On the contrary, it promotes cell survival (Rabinowitz \& White). There is even the case of anticancer drugs such as histone deacetylase inhibitors or temozolomide which induce cell death and are more effective in combination with autophagy inhibitors (Carew et al. 2007). The example of these anticancer drugs also shows that for some time misleading conclusions were drawn from observing autophagy in dying cells. Histone deacetylase inhibitors or temozolomide function as anti-tumour agents by inducing cell death, and because this death is accompanied by increased autophagy it was believed to be autophagic cell death (Kanzawa et al. 2004; Shao et al. 2004). However, the autophagy induced by these drugs was shown to be cytoprotective rather than cytotoxic (Katayama et al. 2007) and this finding was confirmed by the beneficial effect of autophagy inhibitors for the therapeutic use of these agents (Carew et al. 2007). There are a few references for a rather complex interplay between autophagy and apoptosis, and also hints that autophagy may lie upstream, parallel or downstream to apoptotic signalling pathways (Debnath et al. 2005; Akdemir et al. 2006). An example of autophagy working downstream is provided by the silencing of Beclin1 and Atg7 in T-cells, which completely suppressed apoptosis in response to the binding of HIV envelope proteins (Espert et al. 2006). Conversely, overexpression of Atg1, an autophagy-specific kinase, induces higher levels of autophagy and cell death with apoptotic features in Drosophila, and thus seems to be an argument that autophagy lies upstream of apoptosis (Scott et al. 2007). An example of autophagy and apoptosis apparently working together in the killing of the cells are MEFs treated with ER-stress inducing drugs. Under these conditions, apoptosis and autophagy were both involved in a process that led to increased cell death and depended on the death associated protease kinase (DAPK), Beclin1 or 
Atg5 and caspase 3 (Gozuacik et al. 2008). However, it is not easy to interpret the data from these diverse studies. For example, more apoptotic cells after Atg depletion does not necessarily mean that Atg-knockdown caused apoptosis. In experiments with knockdown mouse embryos lacking the mammalian Atg homologues Beclin1, Ambra1 and FIP200 (which are nonviable), the increased number of apoptotic bodies in the embryos was explained by the role of autophagy in clearing up apoptotic bodies (Qu et al. 2007).

The delicate interplay between autophagy and apoptosis also becomes apparent through cleavage events. The most famous example of a protein which switches from being proautophagic to pro-apoptotic is Atg5 when it is cleaved in a calpain-mediated manner (Yousefi et al. 2006). Beclin1 can also be cleaved by caspases, thus resulting in its inactivation and thereby probably inhibiting autophagy as a survival mechanism once apoptosis has been induced (Luo \& Rubinsztein 2010). On the contrary, caspase-cleaved Atg4d becomes a pro-apoptotic factor (Betin \& Lane 2009), similar to cleaved Atg5. These findings point to the notion that caspases and autophagy might have complementary roles in death and survival pathways.

An atypical example of autophagy-related cell death is the indirect induction of death caused by accumulation of cell-damaging reactive oxygen species (ROS). Autophagy is capable of degrading catalases and thereby removing the scavengers of ROS, a phenomenon that can result in cell death. This death was inhibited by knockdown of Atg7, Atg 8 or RIP (Yu et al. 2006). While Atg7 and Atg8 are key proteins in autophagy, RIP, a receptor-interacting-protein kinase, has been associated with autophagy, apoptosis and necrotic cell death, thus further complicating the picture. An explanation for all these seemingly contradictory studies still needs to be found but it seems likely that autophagy plays a role in the pro death-anti death equilibrium. It could be that either up- or downregulated autophagy leads to cell death. Although there are numerous examples of Atg-dependent death and cell loss caused by excessive autophagy induction in cultured cells (Kanzawa et al. 2005; Pattingre et al. 2005), there is no physiological mammalian model for cell loss caused by autophagy in vivo. Yet, if there is such a thing as autophagic cell death, the change from the survival to death mechanism is likely to depend on the type and degree of environmental changes or stimuli. The finding that inhibition of apoptosis can lead to autophagic or necrotic cell death supports the hypothesis that there is a complex interplay between the regulation of both apoptosis and autophagy (Fimia \& Piacentini 2010). 


\section{Autophagy modulators}

\subsection{Autophagy inducers and their relevance for therapy}

The most potent known physiological inducer of autophagy is starvation as stated above (see 5.1.). Most drugs used for autophagy stimulation modulate the nutrient sensing pathways in which the autophagy suppressor mTOR is a suitable target (for more information on mTOR see section 5.1. or figure 2 ). For example, rapamycin and its analogues activate autophagy via inhibition of mTOR in vitro and in vivo (Ravikumar et al. 2004). There are recently developed agents which inhibit mTOR more strongly than rapamycin, such as Torin1, an ATP-competitive inhibitor (Thoreen et al. 2009). Lithium inhibits two enzymes with opposing effects on mTOR signalling, but used in combination with mTOR inhibitors, lithium somehow induces autophagy (Sarkar et al. 2008). BH3mimetics able to interrupt the Beclin-Bcl-2 interaction can also induce autophagy, as shown for example by the competitive inhibitor of this interaction ABT727 (Maiuri et al. 2007). In small-molecule screens for autophagy inducers, many of the compounds found had been already approved for treatment of diseases, as was the case with fluspirilene, trifluoperazine, pimozide, nicardipine, niguldipine, amiodearone, verapamil, minoxidil, perhexiline, niclosamide or clonodine (Zhang et al. 2007; Williams et al. 2008; Balgi et al. 2009).

Since the first screen of this type in yeast (Sarkar et al. 2007), the interest in autophagy inducers has been growing not only for research purposes but also for possible treatment of diseases, especially neurogenerative disorders where an autophagic clearance of neurotoxic products is desirable (Renna et al. 2010).

\subsection{Pharmacological autophagy inhibitors}

For the most part, inhibition of autophagy is only relevant in basic research, although there might be implications for autophagy inhibitors as "add-ons" for anti-cancer drugs that function by inducing tumour cell death and are prone to losing their efficacy due to increasing drug resistance during the course of the disease. This is the case, for example, with tyrosine kinase inhibitors used for treatment of various types of cancer (Bellodi et al. 2009). Resistance to the HER-2 interfering Trastuzumab that is administered in certain types of breast cancer is facilitated by autophagy (Vazquez-Martin et al. 2009). However, despite various studies on this topic it remains unclear whether patients suffering from cancer could benefit from autophagy inhibitors. 
A problem in basic autophagy research is that there are still no highly specific inhibitors of this process and therefore there is an interest in developing such agents. In-vitro inhibitors of the PI3-kinase such as wortmannin (WM) or 3-methyladenine (3-MA) are commonly used to block the class III PI3K Vps34 that is required together with Beclin1 for autophagosome formation (Blommaart et al. 1997). However, PI3K inhibitors also tend to inhibit the class I PI3K that lies upstream of mTOR in the signalling pathway and has an opposite effect on autophagy from the class III PI3K. Additionally, due to the broad impact of PI3K on cell signalling and membrane trafficking, their inhibition is not autophagyspecific. In addition, 3-MA needs to be used at such high concentrations that this affects glycogen metabolism and lysosomal acidification as well as other processes (Caro et al. 1988). There are a few chemical inhibitors which can inhibit autophagosome fusion with lysosomes or degradation in autolysosomes. For instance, as the autophagosomelysosome fusion is microtubule dependent, the microtubule-disrupting agents, vinblastine and nocodazole, inhibit this step (Kimura et al. 2008). Degradation inside the autolysosome is blocked by ammonium chloride or the lysosomal protease inhibitors E64d and pepstatin (Tanida et al. 2005). Bafilomycin A1 inhibits lysosomal acidification rather than autophagosome fusion with lysosomes as was originally reported (Klionsky et al. 2008). As with most pharmacological approaches neither the microtubule nor the fusion/acidification inhibiting agents are specific.

\section{Monitoring macroautophagy}

\subsection{Electron microscopy}

Autophagy was first discovered by electron microscopy, and in the beginning, this technique was the only way to detect and monitor autophagy (Arstila \& Trump 1968). Although the resolution of transmission electron microscopy is superior to fluorescence microscopy it has its limitations, as it can be difficult to diagnose the organelles. Thus, while it is rather easy to detect an autophagosome by means of a double membrane with undigested cytoplasmic contents inside, it becomes more difficult to diagnose hybrid organelles such as the autolysosomes, and to distinguish them from other endocytic compartments. Late autophagic compartments may have fused with endosomes and contain partly degraded material which complicates the distinction between autophagic and endocytic vesicles. Another limitation is the high cost in electron microscopy, usually leading to a small sample size and analysis of just a few ultrathin slices instead of the 
whole cell (Yla-Anttila et al. 2009). An example of an electron microscopy image obtained of autophagic mammalian cells is given in figure 5 .
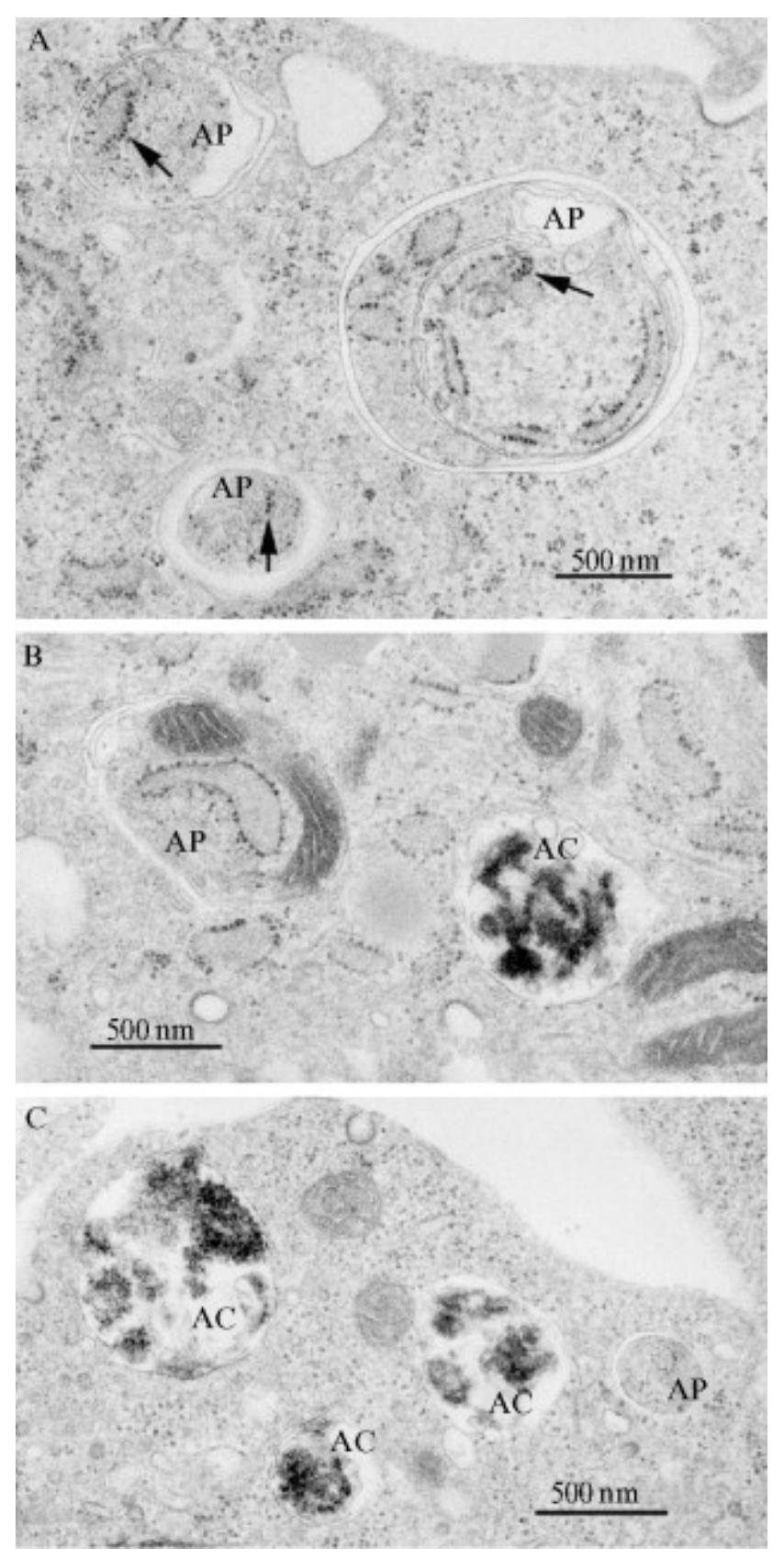

Figure 5: Electron microscopy (Ylä-Anttila et al. 2009)

Fine structure of autophagosomes (early/initial autophagic compartments) and late autophagic compartments (amphisomes and/or autolysosomes) in plastic embedded mouse embryonic fibroblasts. A Three autophagosomes (AP) of different sizes. The large autophagosome on the right has formed around another autophagosome that is now visible inside it. Ribosomes (arrows), either free or attached to ER membranes, are visible inside all autophagosomes. B The autophagosome on the left (AP) contains rough ER and a mitochondrion. The late autophagic compartment (AC) contains partially degraded, electron-dense ribosomes. C The three late autophagic compartments (AC) contain partially degraded ribosomes. One small autophagosome is also visible (AP).

\subsection{LC3 as an autophagic marker}

Unlike most Atg-proteins which can only be observed on phagophores but not on the complete autophagosomes, LC3 is found both on the phagophores and on the inner and outer membrane of the autophagosome. This is mainly the reason why it is widely used as an autophagy-specific marker that labels phagophores, autophagosomes and autolysosomes (Kabeya et al. 2000; Mizushima et al. 2004). As mentioned in section 5.3.1., LC3, the mammalian homologue of yeast Atg8, is cleaved by Atg 4 at its Cterminus to become cytosolic LC3I. During phagophore elongation LC3I is conjugated with phosphatidylethanolamine to produce membrane-bound LC3II. In SDS-page gels, the lipidated form, LC3II, migrates faster than the smaller LC3I, and its levels are a direct measure of the intensity of the autophagic process that occurs in a particular situation. This apparent weight loss of the actually bigger, membrane-bound form, is most likely due 
to extreme hydrophobicity, and should not be misunderstood as processing or another type of size reduction.

A complementary way to detect autophagosome formation, is based on the use of a GFPLC3 fusion protein. GFP-LC3 is detected by fluorescence microscopy and changes from a diffuse cytosolic staining to punctae structures once autophagy is induced (Kabeya et al. 2000). In vivo analysis is possible in this context using GFP-LC3 transgenic mice (Mizushima et al. 2004). A certain degree of unspecific aggregation has been described with overexpressed GFP-LC3, and often the difference between artificial aggregates and autophagosomes is unclear (Kuma et al. 2007). Therefore, high expression levels of the reporter should be avoided. Another option is to use a mutant form of GFP-LC3 which cannot be lipidated as a negative control (Tanida et al. 2008). Due to basic levels of autophagy, at least some autophagosomes should be detectable in all cells, and it therefore is advisable to quantify punctae per cell rather than cells with punctae (Mizushima et al. 2010). Of the four isoforms mentioned above, LC3B is the best studied one. While all isoforms are associated with autophagic membranes they differ in expression pattern and posttranslational modifications (He et al. 2003).

For a graphic description of these events, please refer to figure 6 .

\subsection{Other autophagic markers}

In mammalian cells the number of lysosomes does not correlate well with the number of autophagosomes and therefore staining with lysosomotropic agents like LysoTracker, acridine orange or monodansylcadaverine should not be used to measure autophagic activity. p62 might be used as a marker for macroautophagy as it is degraded within autophagosomes and accumulates when autophagy is inhibited (Bjorkoy et al. 2009). However, a problem with p62 is that it is not clear whether autophagy is the only way by which the protein is degraded. There have been attempts to measure and quantify autophagy by flow cytometry using GFP-tagged LC3 and taking advantage of the fact that the GFP fluorescence is reduced in the autolysome due to the low $\mathrm{pH}$ (Shvets et al. 2008). 


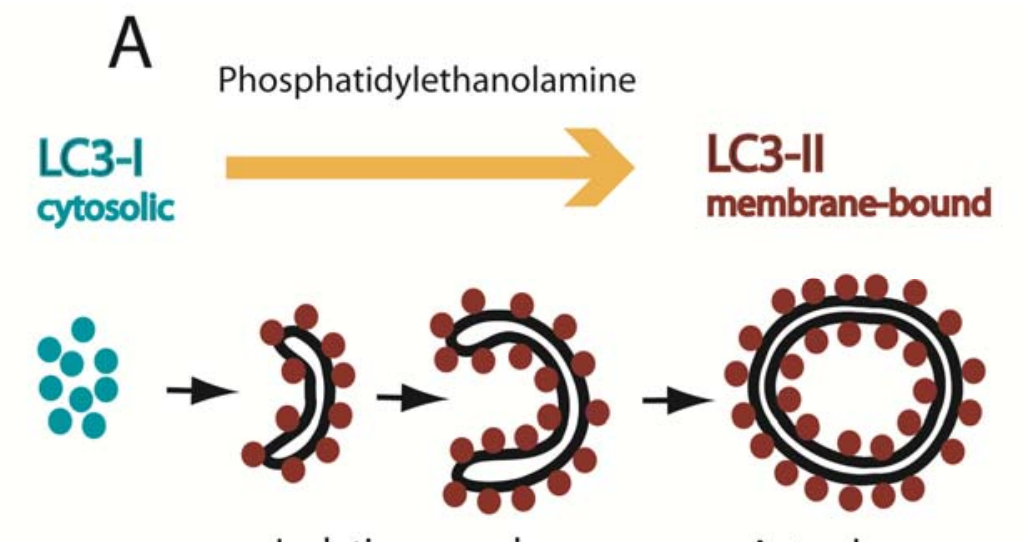

Isolation membrane

Autophagosome

Cytosolic LC3-I — Membrane-bound LC3-II

B

Fluorescence microscopy

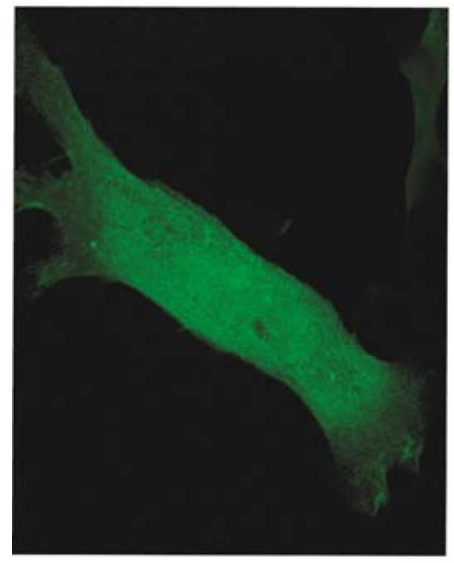

Cytosolic GFP-LC3
$+$

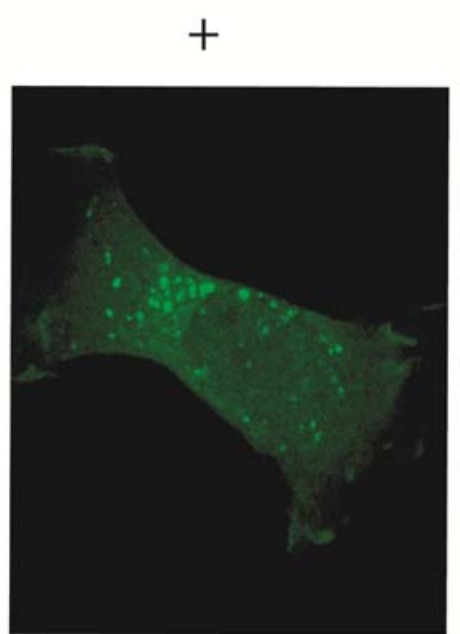

GFP-LC3 punctae
C Western blotting

$-+\quad+$

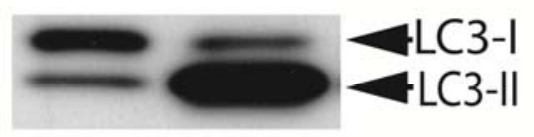

-- basal condition

+ after induction of autophagy

Figure 6: The autophagic marker LC3

A Cytosolic LC3I localises to the isolation membrane upon autophagy induction, thereby it is lipidated by phosphatidylethanolamine and becomes membrane-bound LC3II. Changes in localisation and lipidation of LC3 can be detected by fluorescence microscopy using GFP-tagged LC3 (confocal images in B) and by western blotting (bottom right C). B In the confocal image of SAOS-2 transfected with GFP-LC3 and submitted to autophagy induction, the previously cytosolic LC3I translocates to the autophagosome and appears as punctuate structures. C In Western blotting one benefits from the fact that formation of autophagosomes involves LC3I lipidation, which increases the running velocity of the protein, now called LC3II in SDS-Page. This leads to detection of LC3II at an apparently lower molecular weight than the cytosolic LC3I. 


\section{High-throughput screening for cell death inducers (Alcala et al. 2008)}

The structural and functional characterisation of a protein able to induce autophagy is the subject of this thesis. This protein was identified in a screening of a human cDNA library for cell death inducers.

The concept upon which this screening was based, was that overexpression of death mediators was known to provoke cell death by activating the downstream pathways. Following this rationale, various types of pathway inducers have previously been found by screening, amongst them, for example, pro-apoptotic clones and NFkB or MAPK pathway activators (Albayrak \& Grimm 2003; Matsuda et al. 2003). To implement this cloning procedure, cDNA clones from a human $\mathrm{T}$ cell library were systematically overexpressed and screened for the induction of cell death features. The cDNA library was obtained from interleukin-2 activated T-cells and a total number of 135000 clones was screened by transfection into HEK-293T cells (Alcala et al. 2008).

\subsection{Experimental procedure}

In order to cope with the high clone number involved in this screening, two steps of the process were robotized and the clones were transfected in groups of clones which were just small enough to easily detect the death process caused by a single clone. Cotransfection of library DNA with GFP facilitated detection of the morphological changes associated with cell death using in vivo fluorescence microscopy.

This number of clones per group, one hundred, had been established in previous experiments with known inducers of apoptosis (see figure 7 ). Therefore, with the help of a robotic system, the bacterial clones of the cDNA library were plated out, and colonies were picked and arrayed into 384 well plates with a bacterial growth medium. By dividing these 384 well plates into four squares, pools of 96 clones each were obtained, their DNA was extracted and subsequently transfected together with GFP into human embryonic kidney (HEK)-293 cells containing the $T$ antigen from the simian virus (SV)-40. The $T$ antigen inside the HEK-293T leads to higher expression levels of the transfected DNA owing to a $\mathrm{T}$ antigen-dependent origin of replication present in the library plasmid. As stated above, detection of one positive clone within a group of this size was still possible. On showing positive for morphological changes associated with cell death, these pools were sub-pooled by rows and columns, and their DNA was transfected in a second round. 
Sub-pooling by rows and columns made it possible to find the single positive clone at the intersection of two positive sub-pools (see figure 8 ). Cell death was verified by staining with propidium iodide (PI) (Alcala et al. 2008). PI cannot enter viable cells but stains dead cells as their membranes have become permeable.

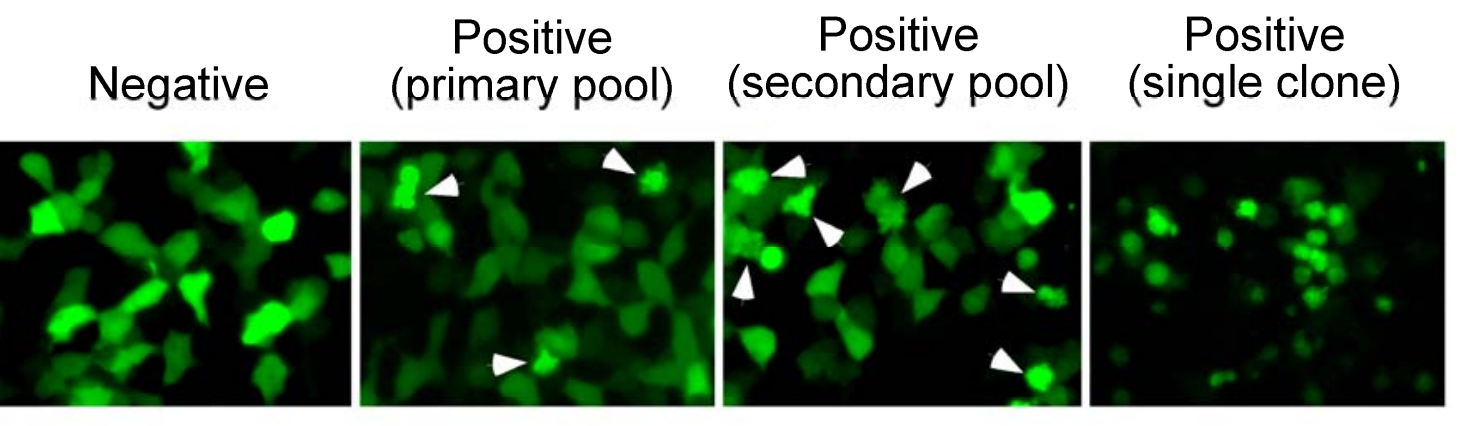

In vivo-20x inverted microscope

Figure 7: Detection of cell death-inducing clones (Alcalá et al. 2008)

The arrows indicate the cells that were transfected with cell death inducers. If the primary pool of 96 clones showed to be positive it was sub-pooled and within the positive secondary pool the single cell death inducing clone could be found. An increase in death-inducing potency can be observed as pool size becomes smaller.

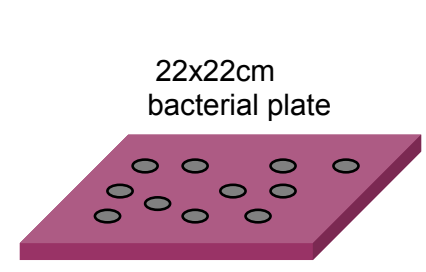

cDNA library clones

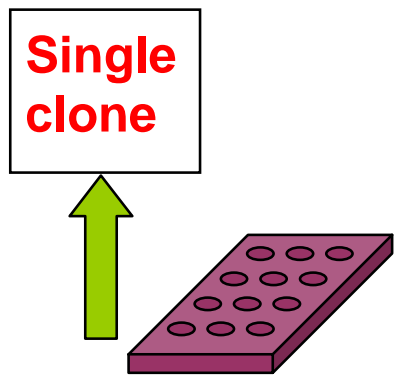

Positive clones are at the intersection of positive rows and columns
384 well plate
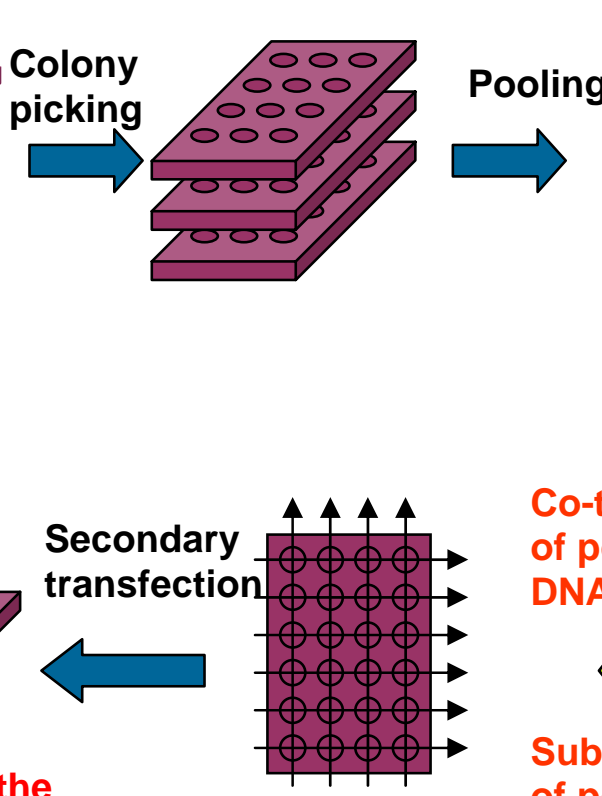

Plasmid isolation of all rows and columns

\section{Growth and plasmid isolation}

96-well DNA preps
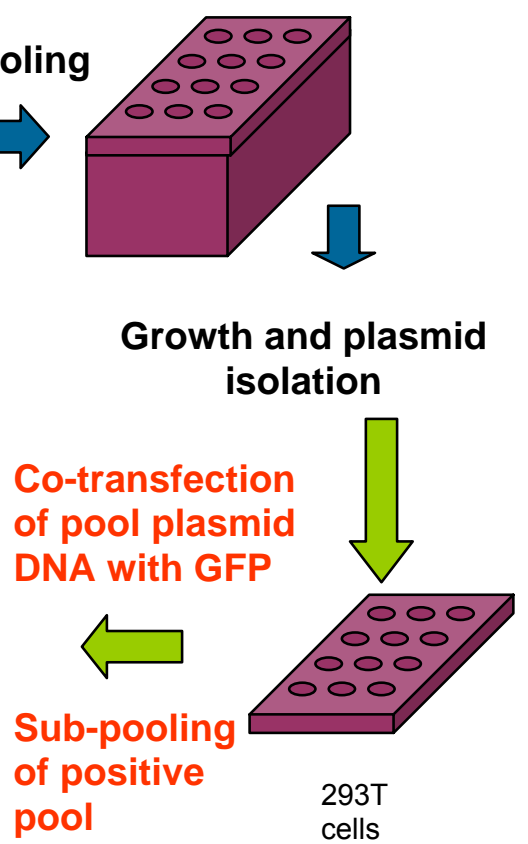
Figure 8: High-throughput screening for cell death inducers (Alcalá et al. 2008)

Expression-cloning scheme. cDNA library clones were arrayed into 384-well plates. Four ninety-six colony pools were subsequently generated from each plate. Pool plasmid DNA was then co-transfected with a plasmid expressing GFP into 293T cells, and death was detected 36h later. Isolation of positive clones was carried out by sub-pooling all rows and columns of each positive pool and co-transfecting the resulting 24plasmid preparations with GFP. Positive clones were to be found at the intersection between positive rows and columns.

\subsection{Identified clones}

Of 229 cell death-inducing clones indentified, 181 were confirmed by positive PI staining. After sequencing and deducting redundant clones, the number of identified cell death inducers remained at 91 . Most gene products induced apoptosis and, not surprisingly, several proteins were well known apoptotic mediators such as for example Bax, caspases and RIP. Apart from apoptosis there were two other types of cell death detected on the basis of the cell morphology that accompanied the death process.

\subsection{Cell death morphologies}

The three different death types were distinguishable on account of morphological changes. The vast majority of clones induced changes consistent with apoptosis such as cell shrinkage and membrane blebbing. The second death type made the cells become detached from the cell plate and rounded like a balloon. These phenomena had previously been linked to autophagic (or type II) programmed cell death (Shimizu et al. 2004). Nearly as many clones induced a type of death that was accompanied by extensive cytoplasmic vacuolisation. This third morphology has been related to a type of cell death called paraptosis (Sperandio et al. 2000). Paraptosis is similar to, but different from apoptosis, and is regulated by an alternative caspase- 9 activity. The general caspaseinhibitor zVAD.fmk partially inhibited cell death induced by the vacuolating clones, whereas it had no impact on the autophagy related death and circumvented apoptosis completely (Alcala et al. 2008) (see figure 9). 

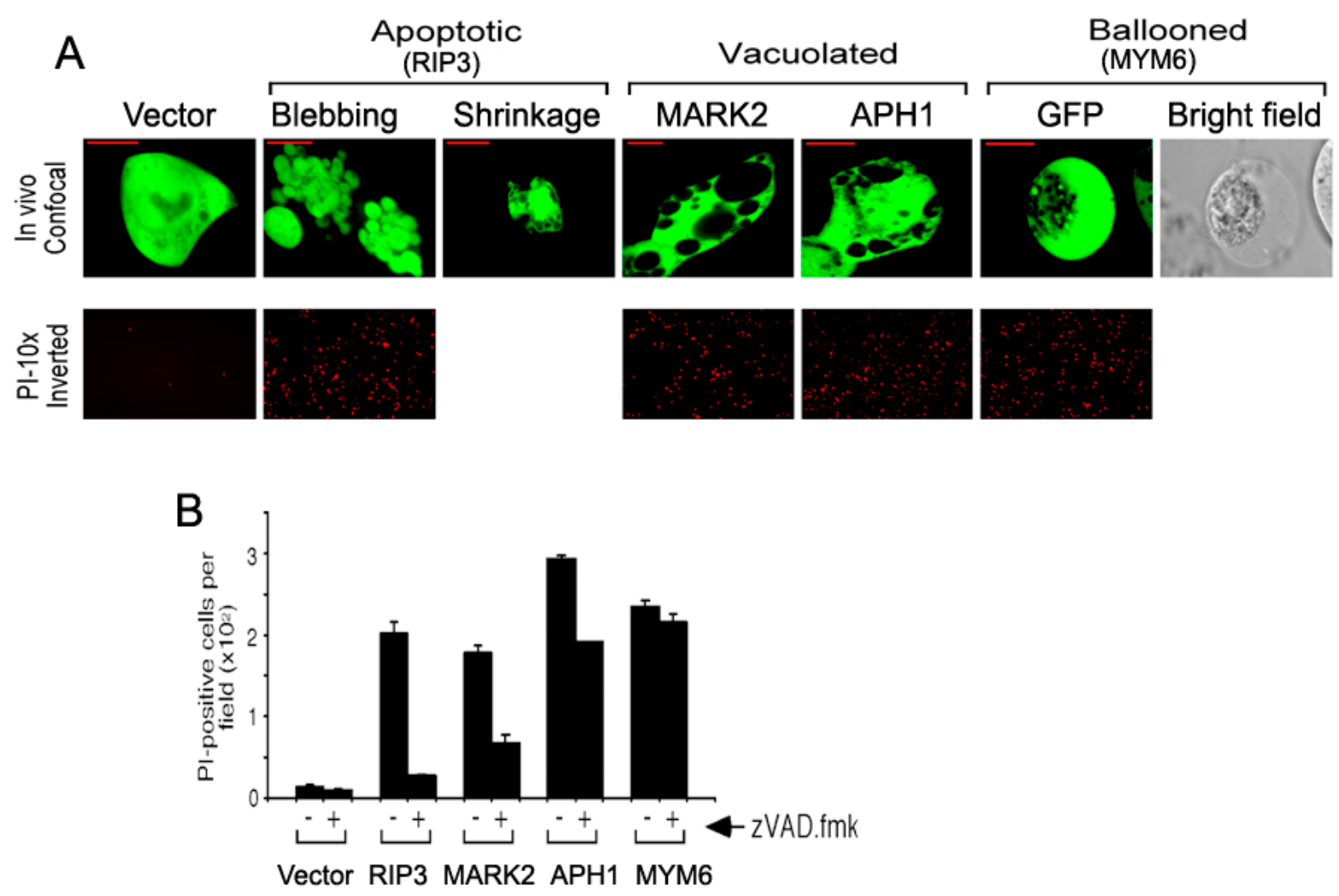

Figure 9: Different types of cell death (Alcalá et al. 2008)

293T cells were co-transfected with the indicated plasmids and GFP. A After $36 \mathrm{~h}$ the dying cells were analysed in vivo by confocal microscopy. There are representative examples of three morphological death types shown in the upper panel: apoptotic, vacuolated and ballooned. In the lower panel the positive propidium iodide staining of the cells undergoing cell death is shown. B Caspase involvement in the different death types. 293T cells were transfected as indicated and treated with zVAD.fmk (50 $\mu \mathrm{M}) 4 \mathrm{~h}$ posttransfection. $36 \mathrm{~h}$ later, the number of propidium iodide $(\mathrm{PI})$-positive cells per field was blindly counted. At least 600 cells were scored for each experimental point. Error bars indicate standard deviations of percentages obtained by counting at least eight different fields.

\subsection{Clone 9-1.4.-P15}

One of the cDNAs identified in this screening, the clone 9-1.4.-P15, induced the ballooned type of death that has been associated with autophagy. This clone appears to encode for a protein with a predicted molecular weight of $35 \mathrm{kDa}$, and seems to have a leader sequence at its $\mathrm{N}$-terminal and a single transmembrane area followed by a potentially intracellular C-terminal region. Based on these observations, the protein could be a type I transmembrane protein and might function as a receptor in the cell membrane. The novel protein, annotated with the systematic name transmembrane protein59 (tmem59), was unknown at the time and showed no obvious homology with other proteins apart from the brain-specific membrane-anchored protein (BSMAP). However, there is very little known about this protein with a highly specific expression for the brain (Elson et al. 1999). 
Recently, it was reported that overexpresion of a cDNA clone that is slightly shorter than 9-1.4.-P15 but expresses the same protein, modulates complex glycosylation and maturation of APP, a central process in Alzheimer's disease (see 6.2.). The authors also showed that this protein is mostly located at the Golgi-apparatus and they further hypothesise that it might modulate $\mathrm{N}$ - and O-glycolsylation of APP and thereby keep it away from the cellular compartments where the secretases involved in APP shedding and $A \beta$ production are located (Ullrich et al. 2010). 



\section{// Objectives}





1. Study whether the properties of the novel protein as an autophagy inducer represent a general phenomenon.

2. Explore the subcellular location of the endogenous protein.

3. Find out which part of the novel protein is responsible for autophagy induction and investigate whether this domain is also sufficient for autophagy induction.

4. Study the involvement of central mediators of the conventional macroautophagy pathway in the autophagic process. 



\section{Results}

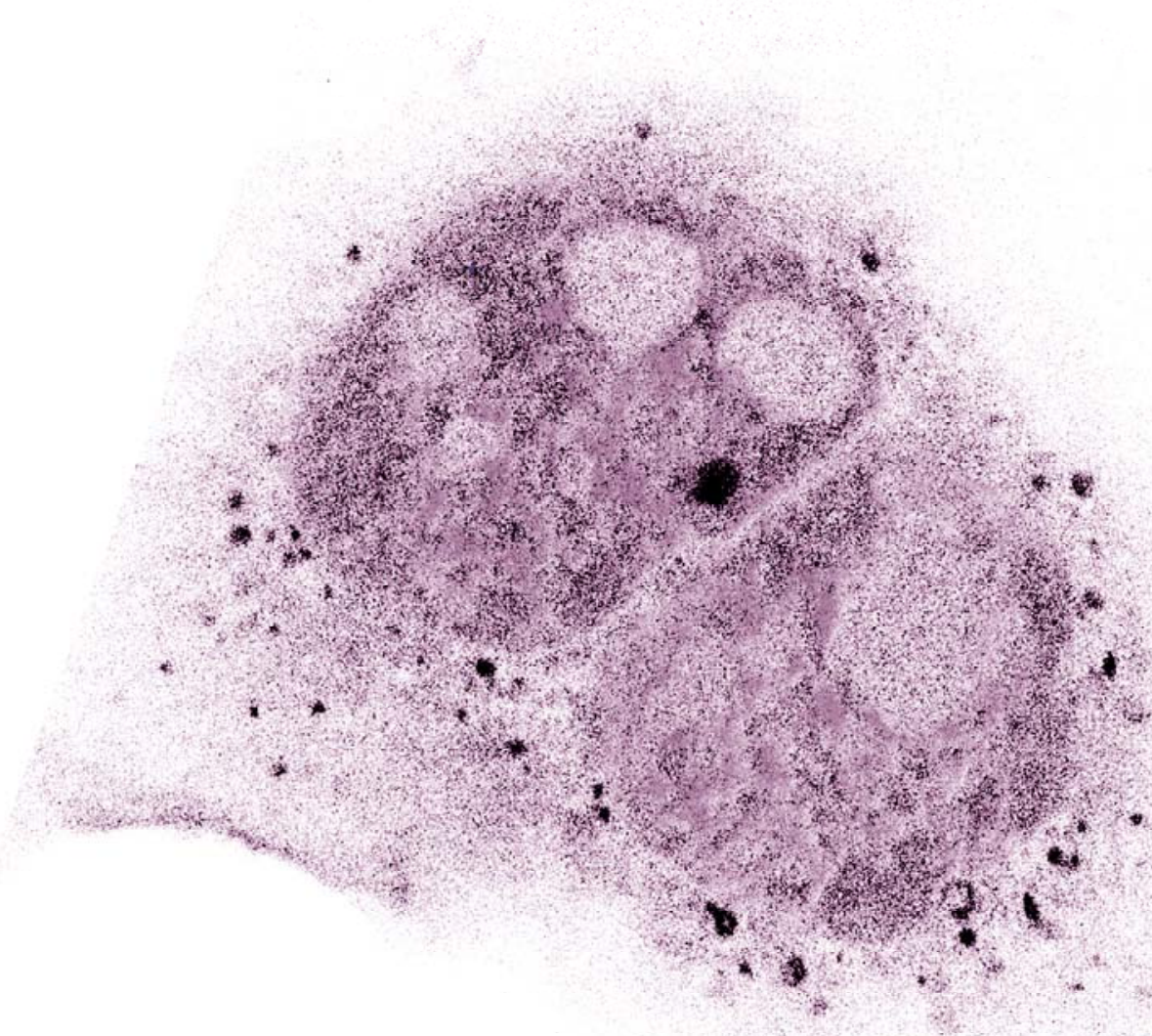





\section{The clone 9-1.4.-P15 induces atypical cell death}

\subsection{Morphology of the death process induced by the novel protein}

As explained in the introduction, the cell death-inducing clones identified in the course of the screening were catalogued by the morphological changes that occurred in the dying cells. These changes were detected by fluorescence microscopy in HEK-293T cells cotransfected with GFP. The novel protein induced a "ballooned" morphology with the cells detaching from the plate and becoming round like a balloon with the cellular content collapsing inside an apparently empty cell (figure 10A). This phenotype could also be visualized by brightfield microscopy and was easily distinguished from the other two cell death phenotypes observed in the screening described earlier: the typical blebbing and shrinkage of cells undergoing apoptosis on the one hand, and a vacuolated morphology where the cell death was accompanied by massive cytoplasmic vacuolisation on the other hand (see figure 9 ).

\subsection{Caspase-independence of the death process induced by the novel protein}

To find out whether the viability of the cells transfected with the newly discovered clone, 9-1.4.-P15, could be restored through inhibiting caspases, the general caspase inhibitor ZVAD.fmk was added to the transfected cells (figure 1OB). After propidium iodide staining, the number of dead cells with and without ZVAD addition was established. The known inducer of apoptotic cell death, RIP3, also identified in the screening, was used as the positive control. Not surprisingly, almost all cells transfected with RIP3 could be rescued from dying by ZVAD.fmk addition. However, the viability of the cells transfected with 91.4.-P15 was not improved by ZVAD.fmk, leading to the conclusion that this type of programmed cell death is caspase-independent.

\section{Measuring autophagy induction by 9-1.4.-P15}

Moreover, this protein led to a type of cell death which showed a ballooned morphology and was insensitive to caspase inhibitors, suggesting that autophagy was involved (Shimizu et al. 2004). To confirm this hypothesis we evaluated the formation of vesicles with double- or multiple membranes by electron microscopy (EM). And in fact, HEK-293 that had been transfected with 9-1.4.-P15 and fixed for EM showed compartments within 
the cytoplasm which had characteristical double- or multiple membranes that are typical of autophagosomes (figure 10C).

In order to further confirm the induction of a canonical autophagic process, the clone 91.4.-P15 was transfected together with human LC3A, once again using HEK-293T cells. As described above in the introduction, LC3 is the most widely used marker to detect and measure autophagy. These experiments were carried out for Western blotting as well as for fluorescence microscopy, and in both cases BNIP3L was used as a positive control for autophagy induction. BNIP3L is known to induce autophagic cell death when apoptosis is suppressed (Kanzawa et al. 2005). To facilitate the detection of transfected LC3, plasmids carrying tagged forms of this protein were used. For the Western blotting experiments, a plasmid expressing HA-LC3 was used. The HA tag in this construct did not only allow the use of an extremely specific antibody, the anti-HA antibody, but the tag made it possible to look solely at the transfected cells which would not have been the case using untagged LC3. Here, the antibody would have detected both, the transfected LC3 and the endogenous LC3 protein from transfected and non-transfected cells. Assuming complete co-transfection between the inducer protein and the reporter plasmid, the changes we see in HA-LC3 are therefore likely to be caused by the co-transfected plasmid. The same happens with GFP-tagged LC3 and, additionally, the GFP tag permitted the detection of the LC3 directly by fluorescence microscopy without first having to stain with antibodies.

In HEK-293T cells transfected with the clone and HA-LC3A, an increase of the lipidated HA-LC3II band which appears beneath the cytosolic HA-LC3I in Western blot could be detected by immunoblotting, and even outranged the band found in the sample corresponding to BNIP3L (Figure 11A). Similarly, in cells transfected with GFP-LC3 the clone led to formation of punctae within the cell while cells co-transfected with the empty vector showed diffuse localisation of the GFP-LC3 in fluorescence microscopy (Figure 11B). The activated LC3II is membrane bound and is found on the autophagosome membranes, thereby being detectable as punctiform structures. These results confirm that transfection of the clone 9-1.4.-P15 leads to autophagy induction. 
A HEK-293 T transfected with P15 and GFP - in vivo confocal images Bright field
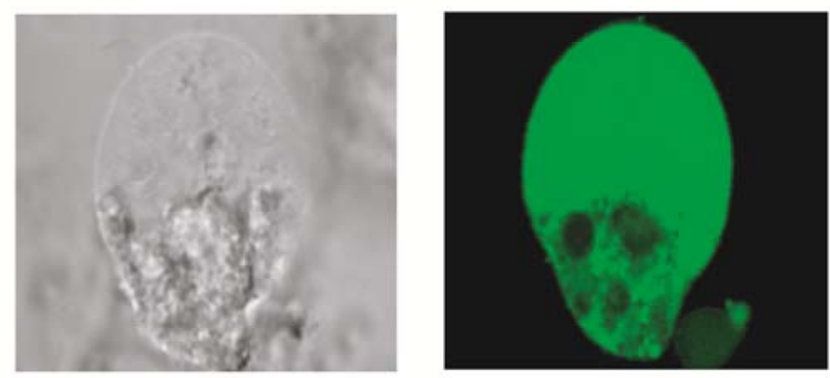

B

HEK-293T transfected with P15 or the apoptotic cell death inducer RIP3

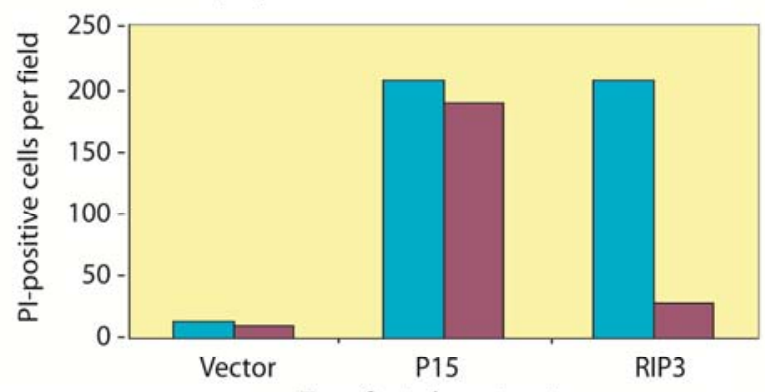

Transfected construct

GFP

$50 \mu \mathrm{M}$ zVAD.fmk
C HEK-293 transfected with P15 - electronic microscopy
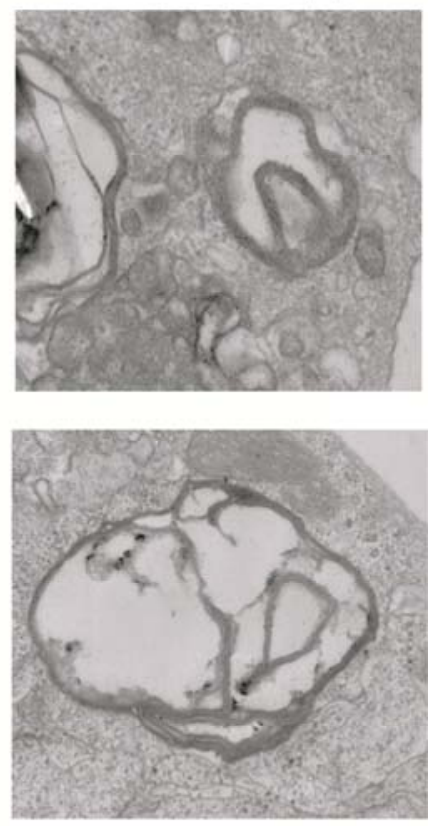

Figure 10: Transfection of the clone 9-1.4.-P15 leads to a non-apoptotic type of cell death.

Samples were analyzed $36 \mathrm{~h}$ after transfection. A While undergoing cell death the cells become detached from their plate and rounded like a balloon. In the co-transfection of the clone with GFP in HEK-293T the dying cells showed a ballooned type of morphology as can be seen in the in-vivo confocal microscopy images. B The cell death induced by P15 is caspase-independent. HEK-293T cells were transfected with the indicated construct and zVAD.fmk was added $4 \mathrm{~h}$ later. $36 \mathrm{~h}$ post-transfection the cells were stained with propidium iodide and counted blindly. At least 600 cells and 8 fields were counted per point. RIP 3 induces apoptotic cell death which therefore can be inhibited by the caspase inhibitor and served as a positive control in this assay. Death caused by transfection of AIR could not be prevented by ZVAD.fmk. C Transfection of AIR leads to formation of double-membraned vesicles. HEK-293 cells were transfected with $\mathrm{P} 15$ and fixed for electronic microscopy $36 \mathrm{~h}$ later. The samples show vacuoles with double- and multiple membranes within the cytoplasm.

\section{9-1.4.-P15 encodes for a transmembrane protein}

As previously stated, the systematic name of the protein encoded by the identified clone is Tmem59, standing for transmembrane protein 59. An alternative name is liver membranebound protein. Sequencing projects have given it the names HSPCOO1 and UNQ169/PRO195. The unprocessed protein is 323 amino acids long and has a predicted mass of $36.2 \mathrm{kD}$. Judging by its predicted structure, 9-1.4.-P15 encodes a type I transmembrane protein with a single transmembrane region and with the $\mathrm{N}$-terminal part in the extracellular compartments and the C-terminal part inside the cell (see figure 11D). Citing the prediction made by Uniprot (www.uniprot.org) from the Swiss Institute of 
Bioinformatics, the first 35 amino acids constitute a signal peptide which is likely to be lost in the mature protein, amino acids 36-238 might be localized in extracellular compartments, amino acids 239-259 represent a potential transmembrane domain and amino acids 260-323 are potentially cytoplasmic. Other databases such as the smart database from Heidelberg (http://smart.embl-heidelberg.de) predict almost the same values. According to this database the transmembrane domain is expected to be within the amino acids $240-262$. Given this putative transmembrane structure and the fact that 9-1.4-P15 is able to provoke autophagy, we decided to call the protein Autophagy Inducing Receptor (AIR).

The subcellular location database, Locate (http://locate.imb.uq.edu.au), from the Australian Institute of Molecular Bioscience at the University of Queensland points out various motifs in the intracellular domain. There are two consensus signals for protein export from the endoplasmatic reticulum (DXE) lying between the amino acids 279-282 and 305-308. There is another motif, YXXL, (amino acids 277-281) which has been shown to be a signal for clathrin- and dynamin-dependent internalization, trafficking of

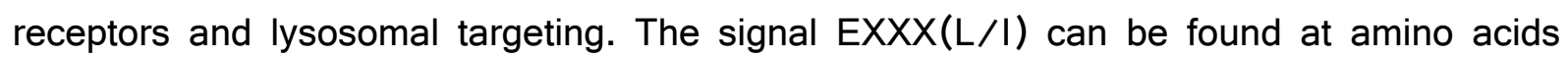
272-276 and amino acids 308-311 and is also listed by Locate as a trans-Golgi-network endosome-sorting motif. This kind of motif is usually referred to as a dileucine motif because of another leucine preceding the last amino acid of this sequence (Pandey 2009). However, there are motifs which have another hydrophobic amino acid instead of a second leucine and therefore the listing of these putative motifs for AIR by Locate seems plausible (Rohn et al. 2000).

\section{A tagged version of AIR is found on the cell surface}

The overall structure of AIR suggests that it might locate to the plasma membrane with a type I topology ( $\mathrm{N}$-terminal extracellular). To gain an indication of whether this was true, HEK-293 were transfected with a form of AIR that was tagged with the HA epitope at the $\mathrm{N}$-terminus and subsequently, surface staining assays with an anti-HA antibody were carried out. As we did not know exactly where the leader of the protein ended and, therefore, where the HA epitope had to be introduced, we chose to fuse our transmembrane protein with the leader sequence of a membrane protein already containing an HA epitope right after the processing site (CD5). After transfection of this construct into HEK-293 cells, these were stained with an anti-HA antibody, thereby omitting prior fixation and permeabilization of the samples in order to mark the surface of the living cell. Interestingly, this leads to strongly stained samples, thus indicating that AIR 
is in fact expressed at the plasma membrane with its $\mathrm{N}$-terminal topologically outside the cell. Although one could argue that this leader from a confirmed cell membrane receptor could have directed the fusion protein to a location other than with its original leader sequence, the positive cell surface staining suggests that our screening-derived molecule is likely to be located on the cell surface in physiological conditions (Figure 11C).

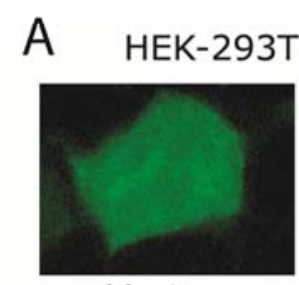

Vector

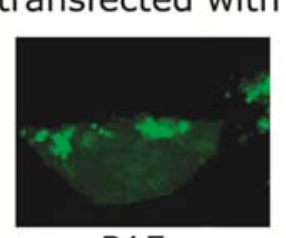

P15

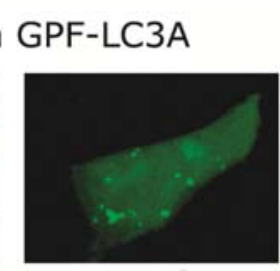

BNIP3L

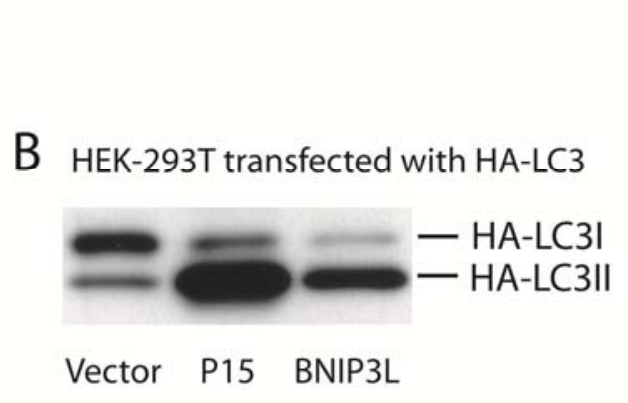

C HEK-293 transfected with HA-tagged P15

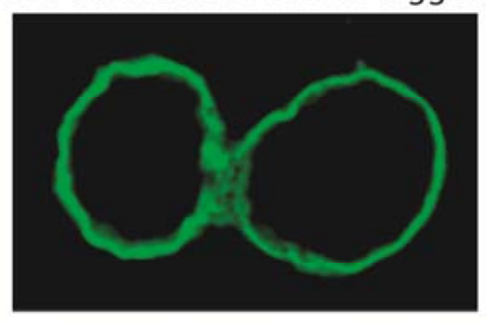

Confocal image of the anti-HA surface staining

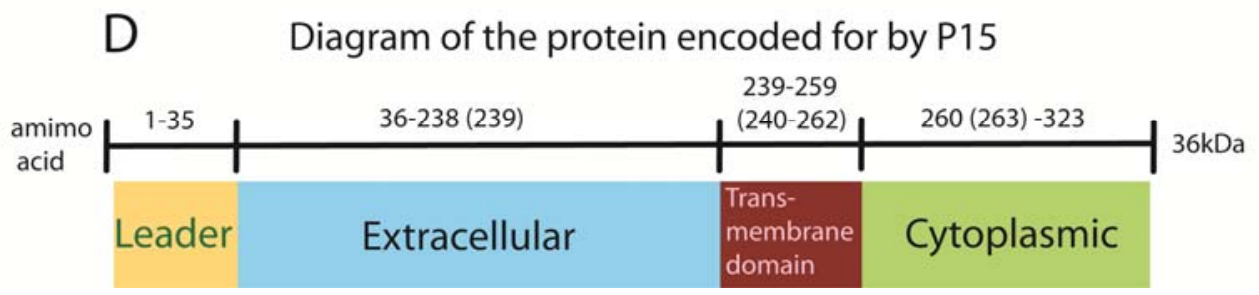

Figure 11: 9-1.4.-P15 induces autophagy.

HEK-293T cells were co-transfected with plasmids expressing GFP-LC3 (A) or HA-LC3 (B) and the empty vector, P15 or BNIP3L. 24h after transfection, the cells were fixed for immunofluorescence $(A)$ or lysed for Western blotting (B). A P15 induces GFP-LC3 punctae formation. In the fluorescence microscopy images, the cells transfected with P15 show GFP-LC3 punctae just as in the positive control (BNIPL3) while the GFP-LC3 in the control is cytosolic. B P15 provokes abundant LC3 lipidation. The Western blot shows even more LC3II in cells transfected with a plasmid expressing P15 than with BNIP3L and in the samples with the empty vector there is hardly any LC3II. C An HA-tagged P15 mutant can be found on the cell surface. HEK293 cells were transfected with a mutant form of 9-1.4.-P15 which contains the leader sequence of CD5 and becomes $\mathrm{N}$-terminally tagged with $\mathrm{HA}$ after leader processing. $40 \mathrm{~h}$ post-transfection a surface staining for HA (green) of the non-permeabilized cells was carried out prior to fixation. The image shows two representative stained cells that were submitted to confocal microscopy. D Schematic description of the novel protein: With a putative $\mathrm{N}$-terminal leader sequence (aa 1-35), a possible $\mathrm{N}$-terminal extracellular part (aa 36-238), a single transmembrane domain (aa 239-259) and the C-terminal predictably being cytoplasmic (aa 260-323), the newly recognised autophagy inducer seems to be a type I transmembrane protein. The numbers show the amino acids which predictably form part of the domain below according to the Uniprot database. The numbers in brackets are alternative amino acids given by the Smart database. 
Homo sapiens (Human) Macaca fascinarius (Crab eating monkey) Bos taurus (Bovine) Sus scrofa (Pig)

Mus musculus (Mouse)

Osmerus mordax (Rainbow smelt)

Salmo salar (Atlantic salmon)

Homo sapiens (Human)

Macaca fascinarius (Crab eating monkey)

Bos taurus (Bovine)

Sus scrofa (Pig)

Mus musculus (Mouse)

Osmerus mordax (Rainbow smett)

Salmo salar (Atlantic salmon)

Homo sapiens (Human)

Macaca fascinarius (Crab eating monkey)

Bos taurus (Bovine)

Sus scrofa (Pig)

Mus musculus (Mouse)

Osmerus mordax (Rainbow smelt)

Salmo salar (Atlantic salmon)

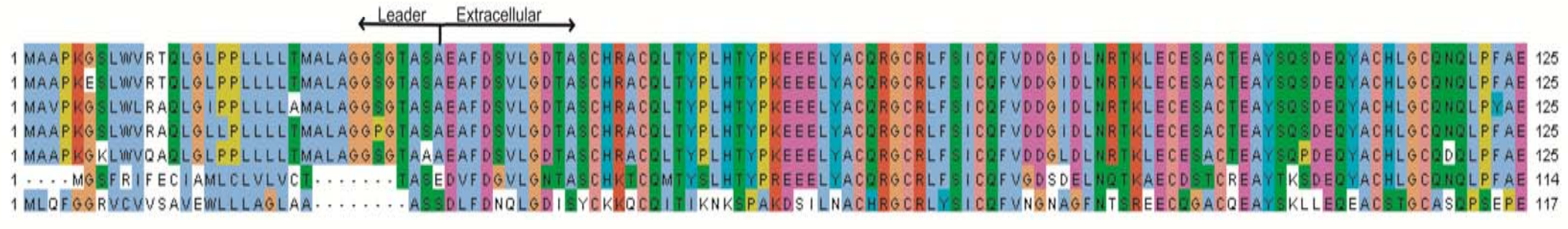
126 LRQEDLM 120 LRQEDLM LMPKM. 20 LROELLMLLMPMHLLFPLTL

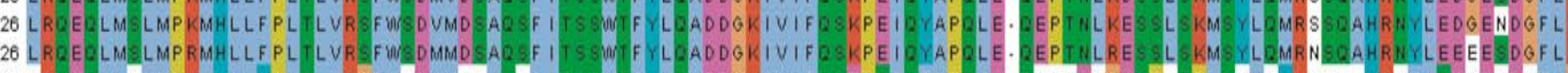
$\ldots \ldots \ldots \ldots+232$

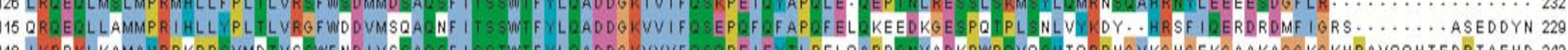

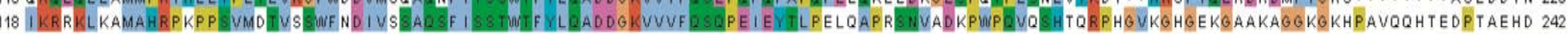
Extracellular Transmembrane $\stackrel{\text { Intracellular }}{\longrightarrow}$

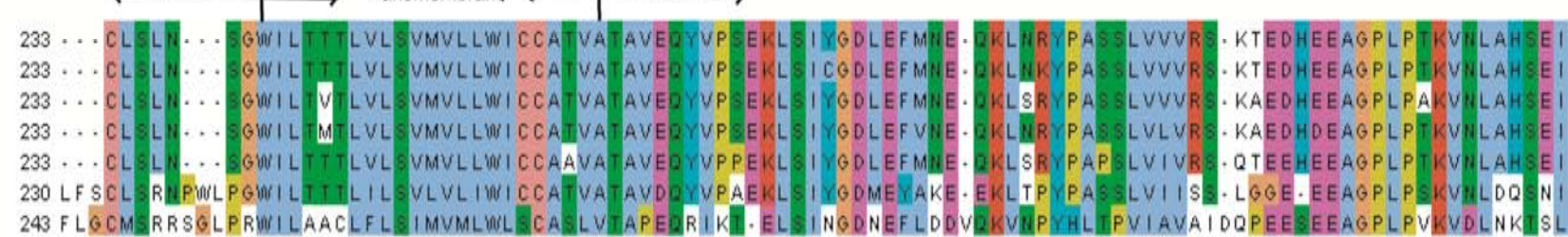

\section{Figure 12: Evolution and conservation of AIR}

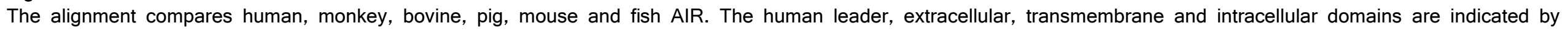

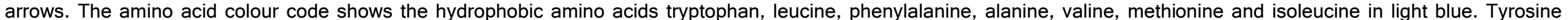

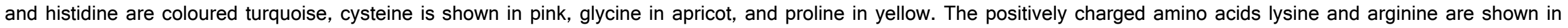

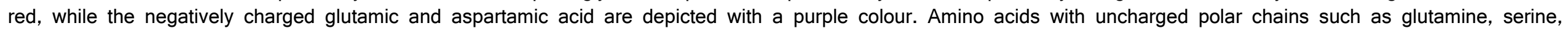
threonine and asparagine are kept in green. The alignment was carried out using clustalX software. 


\section{Conservation of AIR in other species}

AIR encodes for a highly conserved protein from fish to mammals. An especially high level of conservation can be found within its extracellular part following the leader, the transmembrane part and the intracellular part which is closer to the membrane. In lower organisms such as flies, worms or yeast, AIR does not exist (figure 12).

\section{The clone 9-1.4.-P15 induces autophagy not only in HEK- 293T cells}

As the clone had been identified in kidney cells (HEK-293T) especially designed to express high protein levels of the transfected plasmids, we wanted to verify whether AIR could provoke autophagy in cell lines of a different origin, and also at lower levels of protein expression. As previously, BNIP3L served as a positive control for autophagy induction measured using LC3 as a reporter system. There are at least four isoforms of LC3 (see introduction 5.3.1.), and, in particular, LC3B is widely used. After having started our experiments using the LC3A form, we also used LC3B for these additional experiments.

When choosing the cell lines for the following experiments, the most obvious choice was to use normal HEK-293 cells to test whether lower expression levels in the same tissue type continue to provoke autophagy induction. However, it was also important to know whether AIR would induce autophagy in other cell types, too, or if this effect was merely tissue-specific. The human placenta choriocarcinoma cell line Jar and the human osteosarcoma cell line SAOS-2 were derived from two quite different tumours. The SAOS2 cell line additionally shows poor transfection rates and low expression levels, thereby being rather in contrast to the HEK-293T cells and creating a model system with transfected protein levels that are closer to those of endogenously expressed proteins.

AIR expression was able to excite the LC3 autophagic reporters in all tested cell lines (HEK-293, Jar, SAOS-2), and both LC3 isoforms, A and B, gave positive results (figure 13).

AIR provoked an increase of the LC3II band in the anti-HA Western blots and it was even more potent than the positive control (BNIP3L) in activating the reporter. The transfection rates, as shown by the corresponding control GST, were comparable between the clone and BNIP3L. Although in the samples with the negative control, the empty vector, higher 
transfection is detected by means of GST, the amounts of LC3II are still very small. The endogenous actin in the blots illustrates equal loading of the samples (figure 13C).

The images of the fluorescence microscopy show cytosolic distribution of the GFP-LC3 (with hardly any punctae) when the cells are transfected with the empty vector, whilst the AIR or BNIP3L samples show punctuate distribution of the isoforms GFP-LC3A and GFPLC3B. The few punctae in the negative controls can be explained by basal autophagy which leads to constant formation of autophagosomes and therefore some degree of clustered GFP signal. As the transfection induces cellular stress, it could have also caused some extent of autophagy induction and subsequent punctae formation (figure 13A). Consistent with the findings from the Western blotting experiment, counting the punctae per cell in the HEK-293 shows even higher numbers of punctae for the novel protein than for the positive control BNIP3L (figure 13B). This indicates that AIR induces autophagy even more strongly than our positive control.

A

HEK-293

(1)

JAR

SAOS-2
GFP-LC3A
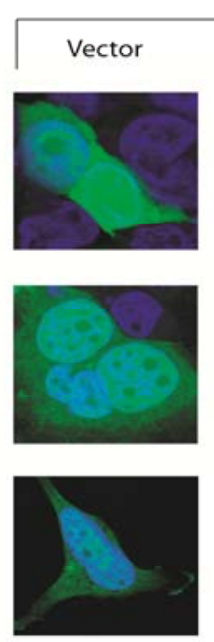

AIR
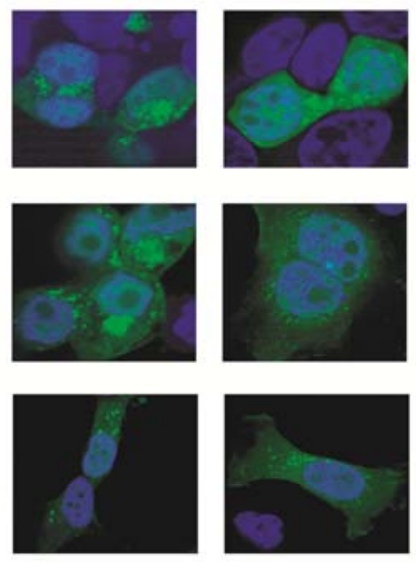

B

GFP-LC3 co-transfected HEK-293

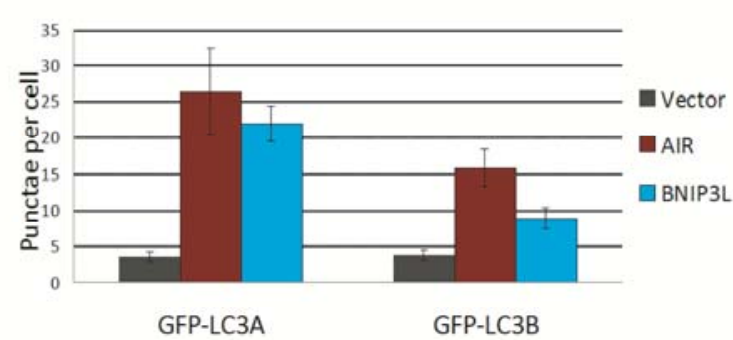

GFP-LC3B
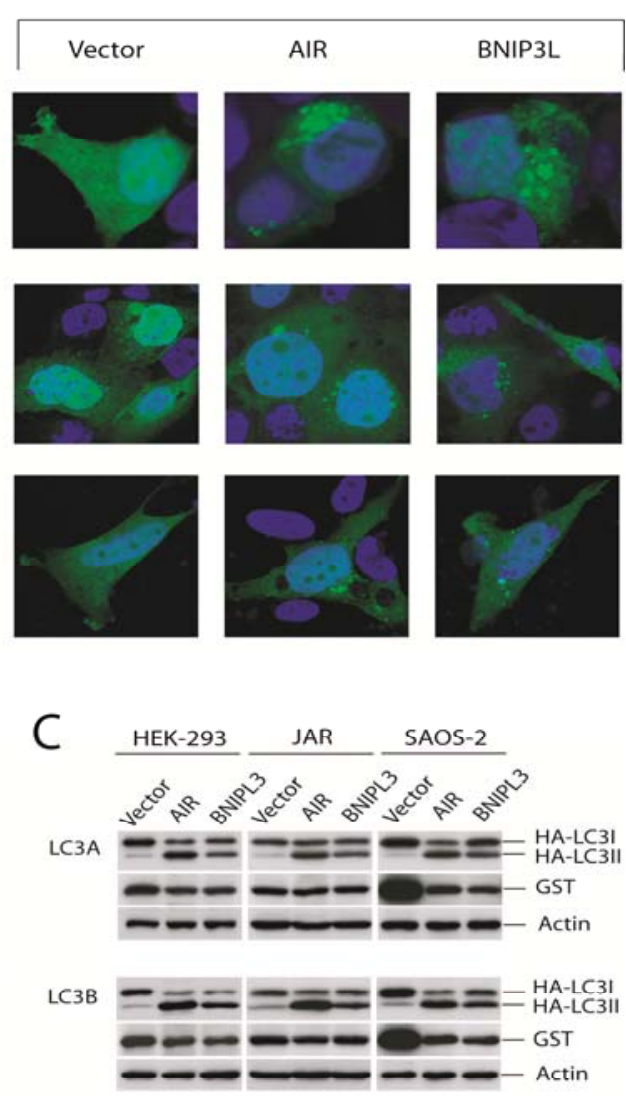

Figure 13: In HEK-293, JAR and SAOS-2 overexpressed AIR excites both isoforms of the autophagy marker LC3.

All cells were analyzed $36 \mathrm{~h}$ post-transfection (fixation for microscopy or lysis for Western blotting). A $A / R$ induces punctae formation of the co-transfected GFP-LC3. The left panel shows representative samples of cells transfected with the GFP-tagged LC3A isoform. GFP-LC3B was used on the right hand side as indicated. Cells expressing an irrelevant vector show cytosolic GFP-LC3 distribution, while there are punctuate structures marked with GFP-LC3 in the samples transfected with AIR or the positive control 
BNIP3L. The cell type for each panel is indicated on the left. B Quantification of GFP-LC3 punctae per cell in HEK-293 underlines the autophagy inducing properties of $A / R$. The experiment was carried out in duplicate and the punctae of at least 70 cells were counted blindly. The left group of the columns represents cells transfected with GFP-LC3A and the right with GFP-LC3B. The grey columns show HEK-293 cells expressing the empty vector, the red columns depict AIR and the blue columns represent the numbers for BNIP3L. C Cells co-transfected with HA-tagged LC3 isoforms show abundant lipidation of LC3 after transfection with AIR for Western blotting. In cells transfected with the empty vector there is hardly any LC3II detectable, while the samples with AIR or BNIP3L show a strong band of the lipidated LC3 form which is even larger in AIR than in BNIP3L. A GST plasmid was co-transfected as a transfection control and proves that the differences in the LC3II band are not due to uneven transfection rates. Actin serves as a loading control.

\section{Antibodies against AIR}

Given that there are no commercially available antibodies against AIR, we had animals of two different species (rabbit and chicken) immunised against a GST fusion protein containing the predicted extracellular domain of AIR, and we purified their antibodies by affinity chromatography against the same protein. Unfortunately, none of these reagents could detect endogenous AIR in the Western blots with lysates from any of the tested cell lines, HEK-293, HCT-116, SAOS-2, HeLa and Jar. However, it seemed that the antibodies could detect ectopically expressed AIR as a band of approximately $32 \mathrm{kDa}$ appeared in Western blots after transfection with an AIR-expressing plasmid (figure 14A). The difficulties in detecting the endogenous protein suggest a rather low level of expression.

\subsection{Detection of AIR by immunoprecipitation}

In order to detect endogenous AIR in cell lysates, total cell lysates were subjected to immunoprecipitation (IP) with the rabbit anti-AIR antibody. With the anti-AIR antibody now being present in the samples loaded onto the SDS-gel, an antibody from a species other than rabbit had to be used for subsequent Western-blotting detections. Therefore, we used our second anti-AIR antibody generated in chicken for Western blot detection of the protein. In this way a band at the expected molecular weight of AIR appeared in the samples of HEK-293, HCT116, SAOS-2, HeLa and Jar cell lysates incubated with the rabbit AIR antibody and protein G coupled to agarose beads. In samples that had only been incubated with the resin, no such band was detectable (figure 14B). To confirm the specificity of the antibodies, SAOS-2 and HeLa were transfected with siRNAs to knock down AIR and subjected to IP followed by WB. And in fact, the detected $32 \mathrm{kDa}$ band disappears in cells which were transfected with siRNA against the newly found autophagy 
inducer but not in those transfected with control siRNA, thus indicating that that we are actually visualizing AIR (figure 14C).

\subsection{Detection of AIR by immunofluorescence}

As AIR seemed to be expressed at a very low level, and showed the highest signal in IP experiments when using SAOS-2 cells, we chose these cells to evaluate the subcellular localisation of endogenous AIR by immunofluorescence. In assays using the rabbit antibody the protein was detected as small dots in the cytoplasm of the cell. Furthermore, the signal proved to be specific as it disappeared after transfecting with siRNA, while the punctuate structures formed by AIR remained in the cells transfected with a control siRNA (figure 14D).

The anti-AIR antibody was also tested in SAOS-2 cells that had been retrovirally transduced to stably express a C-terminally HA-tagged AIR construct. In contrast to transfection, infection usually leads to transduction of almost all cells (especially if followed by selection with the appropriate selection medium) but lower expression levels of the relevant protein. These SAOS-2-AIR-HA cells were either stained with the rabbit anti-AIR antibody or with an anti-HA antibody. Not surprisingly, there were more AIR punctae detected in SAOS-2-AIR-HA cells than non-transduced cells. Additionally, the anti-HA antibody seemed to be more sensitive than the AIR antibody as there were more AIR punctae in HA stained cells than in AIR stained cells. However, the differences in detection could also be due to the fact that the anti-HA antibody is monoclonal and detects the C-terminal part of the protein while the anti-AIR antibody is polyclonal and detects the N-terminus of AIR. Transfection with siRNAs showed once again that the antibodies were specific, and it was also demonstrated that knock-down of ectopically expressed AIR was possible, the latter being confirmed by IP and WB analysis (figure $14 \mathrm{E})$. 
A Detection of transfected AIR in HEK-293 by Western blotting

WB: rabbit anti-AIR

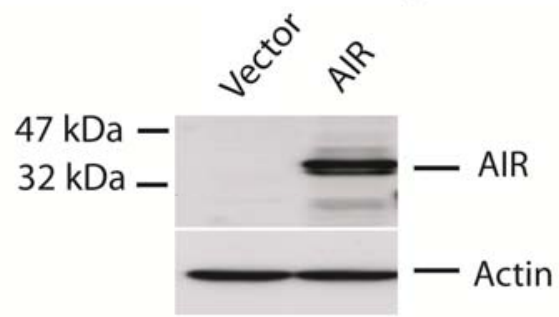

B Detection of endogenous AIR by Western blotting in different cell lines after immunoprecipitation

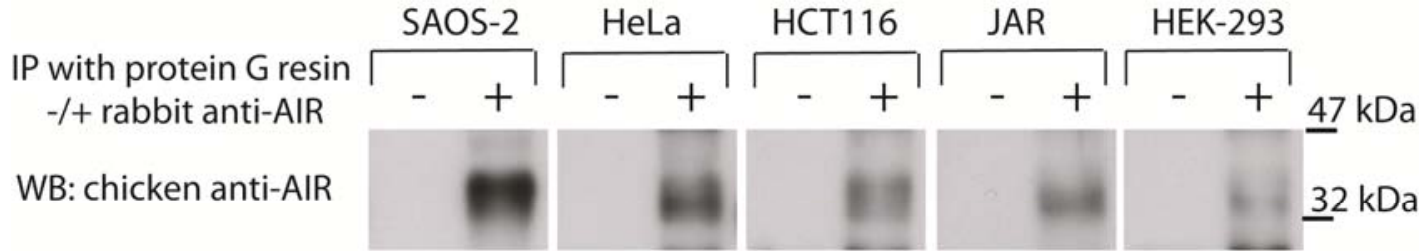

C Specificity of AIR antibody is backed up by experiments with siRNAs

IP: rabbit anti-AIR WB: chicken anti-AIR

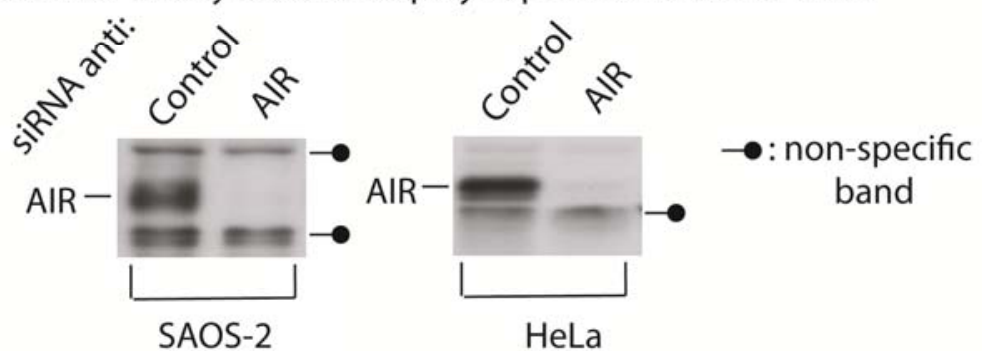

D AIR antibody in SAOS-2 cells transfected with siRNA against AIR

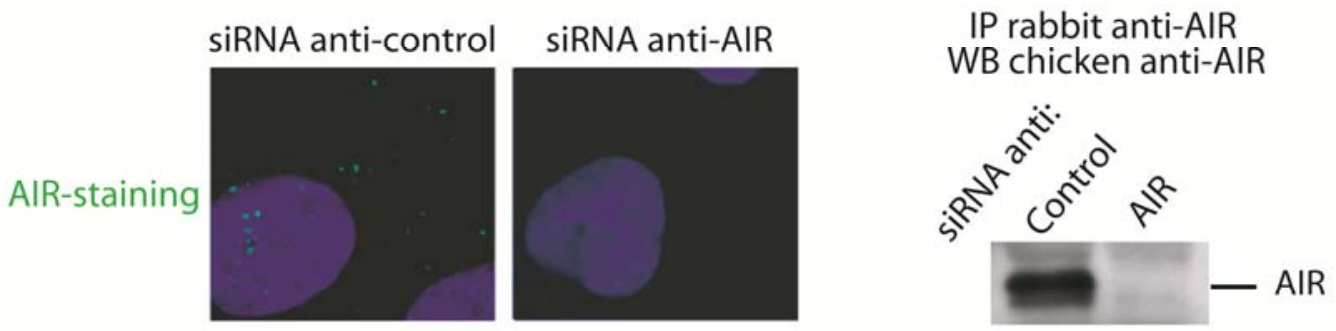

E siRNA in SAOS-2 stably transfected with AIR-HA and detection of AIR

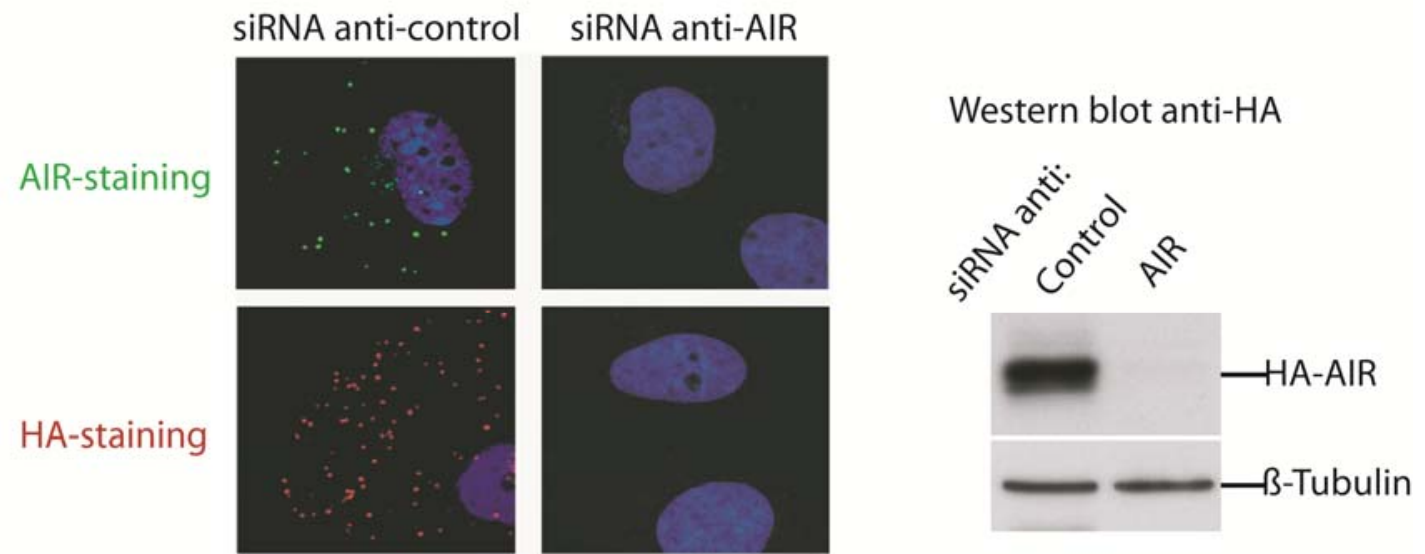


Figure 14: AIR can be detected with specific antibodies.

A Transfected AIR is easily made visible by immunoblotting. HEK-293 were transfected with AIR or the empty vector and lysed $40 \mathrm{~h}$ later. For detection of the protein after Western blotting, a purified rabbit antiAIR serum was used. B Endogenous AIR can be detected after immunoprecipitation. The full lysates of SAOS-2, HeLa, HCT116, Jar and HEK-293 cells were submitted to an immunoprecipitation with protein G resin and the rabbit anti-AIR antibody. After running a SDS-page with the samples, a chicken anti-AIR antibody was used for the Western blotting detection. The blot shows samples which had been incubated with protein $\mathrm{G}$ resin in the presence and absence of the rabbit anti-AIR antibody and only the lanes with the antibody-treated samples show a band at the expected molecular weight of AIR. C The anti-AIR antibodies are specific. SAOS-2 and HeLa cells were transfected with SiRNA against AIR or control siRNAs without any target RNA in human cells. These cell lysates were submitted to an IP as described in B. The Western blots demonstrate that the broadest chicken anti-AIR band disappears in cells transfected with siRNA against AIR. However, there are one (HeLa) or two (SAOS-2) smaller non-specific bands that do not disappear after AIR knock-down. D Endogenous AIR locates to small cytoplasmic vesicles. Green punctuate structures are observed in the cytosol of SAOS-2 cells after fixation and staining with the rabbit antibody. As demonstrated by a siRNA experiment these punctae are specific for AIR. The corresponding IP followed by WB (see B) for the siRNA experiment shows that AIR has been knocked down successfully and the antibody is specific. E Ectopically expressed AIR apparently shows a similar distribution pattern compared to endogenous AIR. SAOS-2 stably expressing HA-AIR were fixed and stained with either anti-AIR or anti-HA for IF. The signal was specific as could be shown by knocking down endogenous AIR and ectopic HA-AIR with siRNA. At the same time it was demonstrated by IP/WB that the siRNA functioned well enough to knock down AIR even in stably AIR-expressing cells

\section{Subcellular localisation of AIR}

Having optimized the conditions to detect endogenous AIR, we could investigate in which cell compartments the novel protein was located. After discovering an $\mathrm{N}$-terminally HAtagged version of AIR to be expressed on the cell surface (see section 4.), the first assumption for the whereabouts of AIR was the cell surface. Consistently, HEK-293 cells transfected with untagged AIR showed a positive surface staining after using the rabbit anti-AIR antibody (figure 16A). By reproducing the detection of AIR at the plasma membrane with the native construct, we also resolved the doubt arising from the use of a different leader in these preliminary experiments (figure 11C). The previous usage of the CD5 leader to artificially introduce an HA tag had not changed the localisation of transfected AIR, which is anyway found on the cell surface when overexpressed in HEK293 cells.

\subsection{Co-stainings of AIR and cell compartment markers}

Recently published data suggests that AIR can be found in the Golgi apparatus (Ullrich et al. 2010), and we therefore investigated this possibility. In double staining immunofluorescence assays with SAOS-2 cells we explored whether AIR colocalised with a protein that is predominantly expressed in the Golgi apparatus, GM130. In our experiments, AIR did not colocalise well with this marker indicating that AIR is expressed in other compartments. 
As we expected AIR to be endocytosed, we thought it could be found in endosomal compartments. Carrying out the same experimental setting as described above we used markers of lysomal compartments such as CD63 or LAMP2. In these experiments, we found considerable colocalisation of AIR with these proteins (figure 15). Therefore we deduce that AIR is mainly localised in the endosomal-lysosomal compartments of the cell.

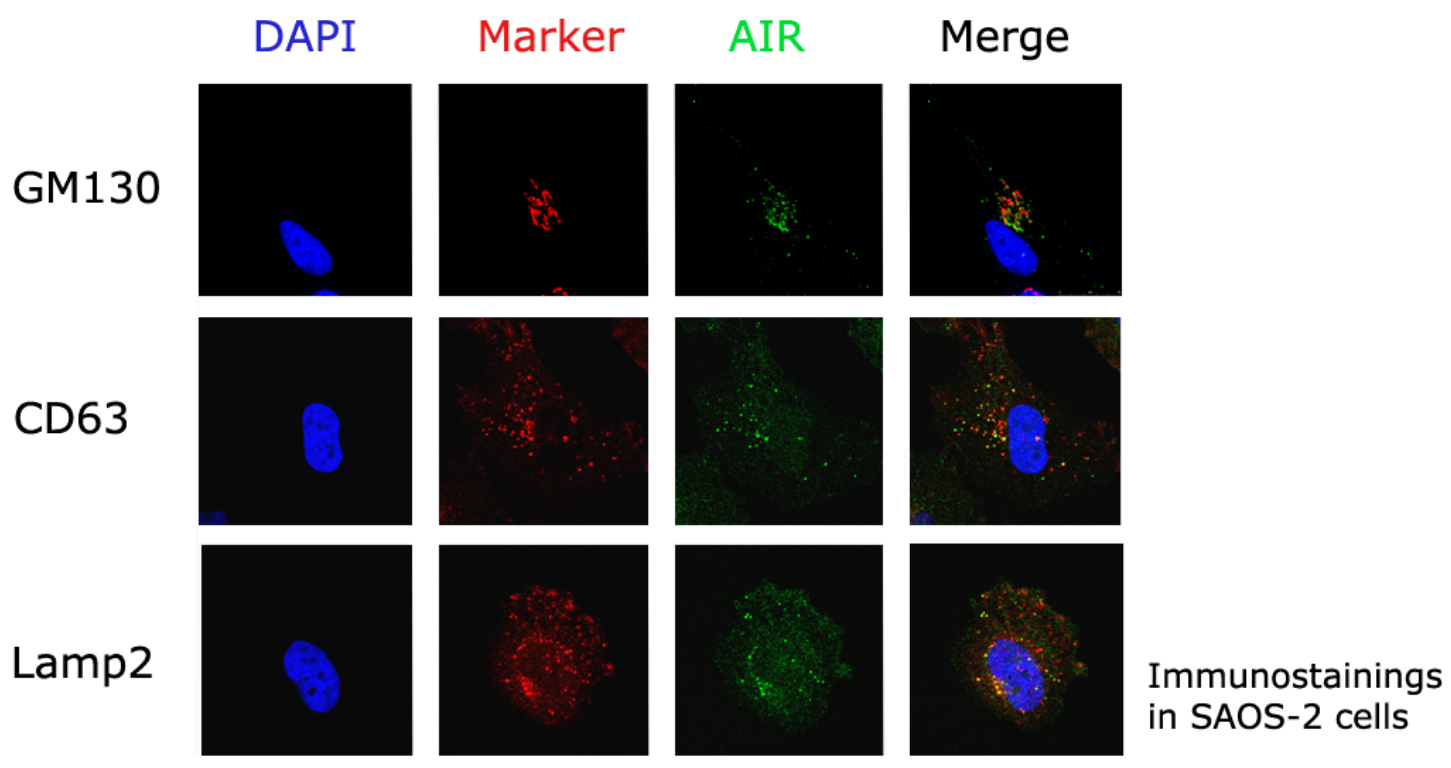

Figure 15: Endogenous AIR localises to late endosomes / lysosomes.

Cells were fixed and permeabilised before incubating them with an anti-AIR antibody (green) and a second antibody against the indicated marker (red). The nuclei were stained with DAPI. The samples were mounted and confocal images were taken of representative cells. The overlays of the red and green channels show where the marker and AIR colocalise. While there is little coincidence between GM130 and AIR, CD63 and Lamp2 seem to be located in the same punctuate structures as AIR when considering the fusion of the AIR and marker images which shows many yellow punctae.

\subsection{Endogenously expressed AIR transiently localises to the plasma membrane}

Although transfected AIR was found on the cell surface, repeated experiments designed to test if endogenous AIR could also be found in such compartments were unsuccessful (data not shown). As mentioned in section 3., AIR carries an endocytosis motif within its putative intracellular part and we considered that the lack of detected AIR on unpermeabilised cells was due to its rapid internalisation. To test this possibility, we treated cells with endocytosis inhibitors prior to carrying out anti-AIR stainings on the cell surface. Chloroquine is an inhibitor of the endosomic pathway that suppresses acidification of the late endosomes. Chloroquine was shown to increase the presence of an endocytosed protein on the cell surface due to inhibiting its transport from endosomes to 
lysosomes and thereby changing the distribution of the protein within the cell (LippincottSchwartz \& Fambrough 1987). Consistently, after incubating the SAOS-2 cells or SAOS2-AIR-HA cells with chloroquine, endogenous AIR or AIR-HA could be detected on the cell surface using anti-AIR antibodies. This made us believe that AIR transiently localises to the cell membrane but is constantly and rapidly endocytosed, thus resulting in a predominant steady state distribution at the endo-lysosomal compartments (figure 16 $B / C)$.

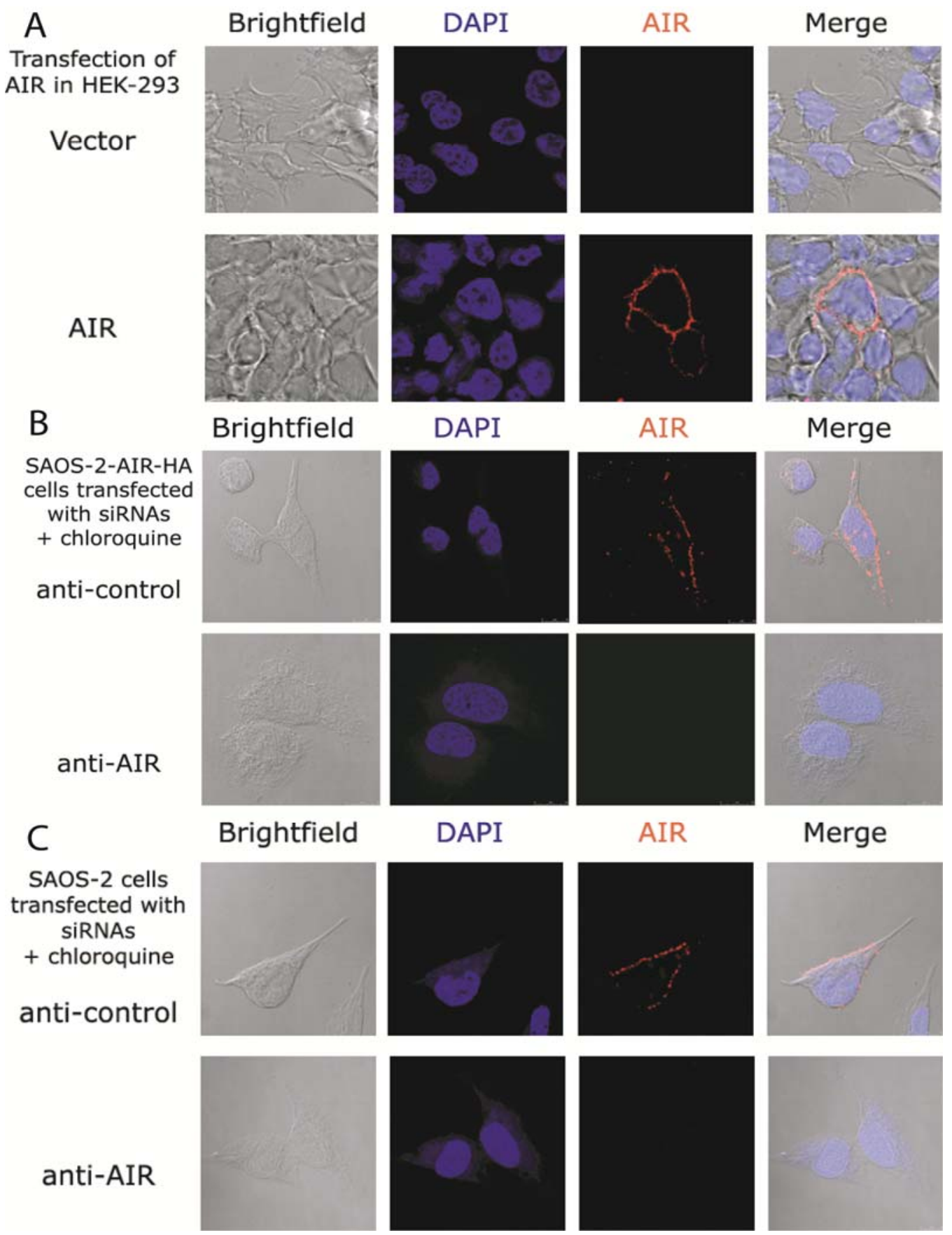


Figure 16: AIR can be found on the cell surface.

A Transfected AIR locates to the cell surface. HEK-293 were transfected with AIR and 4Oh later they were submitted to a cell surface staining using the rabbit anti-AIR antibody. The staining was carried out without prior permeabilization. During the staining process the cells were kept at $4^{\circ} \mathrm{C}$ and in the presence of sodium azide until fixed and mounted for confocal and brightfield microscopy. AIR is found on the cell surface as can be seen in the brightfield/confocal images overlay of the anti-AIR staining. The cell nuclei were made visible using DAPI. B Stably expressed AIR localises to the cell surface in the presence of chloroquine. SAOS-2 stably expressing HA-AIR were transfected with anti-control or anti-AIR siRNA, 48h later they were incubated with chloroquine $(50 \mu \mathrm{M}, 24 \mathrm{~h})$ and stained for AIR as described in $\mathrm{A}$. In contrast to the cells transfected with anti-AIR siRNA, the anti-control siRNA cells show positive for AIR on the cell surface which is illustrated in the brightfield/confocal images overlay. C Endogenous AlR locates to the cell surface in the presence of chloroquine. WT SAOS-2 were subjected to the same experiment as in B which also made it possible to detect endogenous AIR on the cell surface. All pictures were taken of representative cells within the samples.

\section{Mapping the active subdomain of AIR}

Having demonstrated that over-expressed AIR induces autophagy in various mammalian cell types, we wanted to know which part of the molecule was necessary for this effect. For this purpose, we made a C-terminal deletion of AIR lacking the predicted intracellular part from aa 263-323 ( $\Delta I D)$. This plasmid was transfected together with the autophagy reporters HA/GFP-LC3A or HA/GFP-LC3B and the autophagic signal was compared with the full length construct. Once again, we tested the cell lines HEK-293, Jar and SAOS-2 and found the same results in all three: the intracellular domain (ID) is needed for autophagy induction. The $\triangle I D$ mutant hardly led to the detection of a LC3II band in immunoblotting or GFP punctae formation in fluorescence microscopy (figure 17A, C). Looking at the Western blot results one can see an almost disappearing LC3II band in cells transfected with the AIR- $\triangle I D$ mutant in comparison with full length AIR (AIR-FL) (figure 17C). Also, in the samples transfected with AIR- $\Delta I D$ for fluorescence microscopy, one detects very few GFP-LC3 punctae while in the same experiment, transfection of the full length plasmid results in abundant punctae formation (figure 17A). Quantification of the number of GFP-LC3 dots per cell in HEK-293 co-transfected with AIR or AIR-AID verified the result that the intracellular domain of AIR is necessary for autophagy induction (figure 17B). 

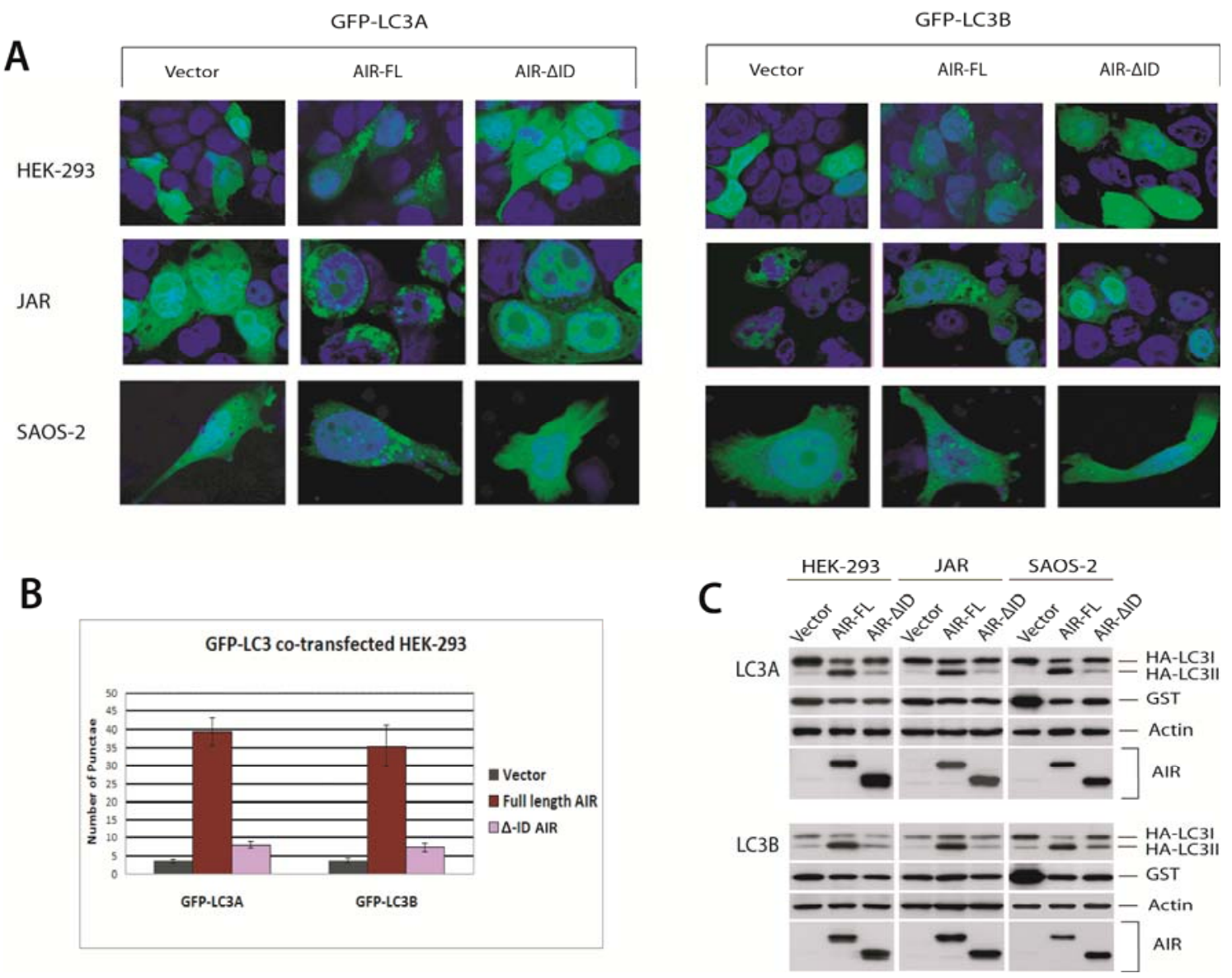

Figure 17: The intracellular part of AIR is necessary for autophagy induction in HEK-293, Jar and SAOS-2 cells.

All cells were analyzed $36 \mathrm{~h}$ post-transfection (fixation for microscopy or lysis for Western blotting). A. Without the intracellular domain of AIR (AIR-ID), the co-transfected GFP-LC3 remains cytosolic. The left panel shows representative samples of cells transfected with the GFP-tagged LC3A isoform. GFP-LC3B was used in the experiments displayed on the right hand side. With the empty vector, co-transfected cells show cytosolic GFP-LC3 distribution and with the AIR-FL construct punctuate structures form, but there are hardly any punctae in samples with the AIR- $\triangle I D$ mutant. B Quantification of GFP-LC3 punctae per cell in HEK-293 underlines the importance of AIR-ID for GFP-LC3 vesicle formation. The experiment was carried out in duplicate and the punctae of at least 70 cells were counted blindly. The left group of the columns represents cells transfected with GFP-LC3A and the right with GFP-LC3B. The grey columns depict HEK293 expressing the empty vector, the red columns stand for AIR-FL and the lilac columns represent the numbers for AIR- $\triangle I D$. C AIR-ID is necessary for lipidation of LC3 in Western blots. The cells were cotransfected with HA-tagged LC3 isoforms A and B. As expected, in cells transfected with the empty vector there is hardly any LC3II detectable, while the samples with AIR-FL show the lipidated LC3 form. However, transfection with AIR- $\triangle I D$ did not lead to this kind of LC3II formation. As previously, the GST plasmid proves that the differences in the LC3II band are not due to uneven transfection rates. Actin serves as a loading control.

\subsection{AIRA 282 remains fully active}

To narrow down the active domain even further, we generated serial C-terminal AIR deletions by shortening the full length protein in steps of five to ten amino acids. The deletions used in this experiment were AIR $\Delta 268, \operatorname{AIR} \Delta 273, \operatorname{AIR} \Delta 282, \operatorname{AIR} \Delta 290$, AIR $\Delta 299$, AIR $\Delta 307$, AIR $\Delta 317$ and AIR $\Delta 319$. The smallest deletion, AIR $\Delta 319$, had been 
designed as lacking precisely the C-terminal amino acids S-E-I that represent a putative pdz-domain interacting motif. Pdz domains are named after the first proteins in which they were found (PSD95, DIg and ZO-1) and play a role in directing the specificity of receptor tyrosine kinase-mediated signalling involved in, amongst other processes, protein trafficking. Pdz proteins bind to molecules that contain the signature $S / T-X-V / I$ at the very C-terminus (Songyang et al. 1997). However, after transfecting the AIR mutants with the autophagy marker in HEK-293, we saw that the SEI peptide had no influence on autophagy induced by AIR since $\Delta 319$ was equally able to provoke LC3 conversion to LC3II as the full length molecule (data not shown). The deletions remained active up to AIR $\Delta 282$, while AIR $\Delta 268$ and AIR $\Delta 273$ failed to induce autophagy just as it was the case for AIR- $\Delta I D$, the deletion lacking the entire intracellular domain from the previous experiment (figure 18). These data indicate that the active subdomain of AIR is found between aa 263-281.
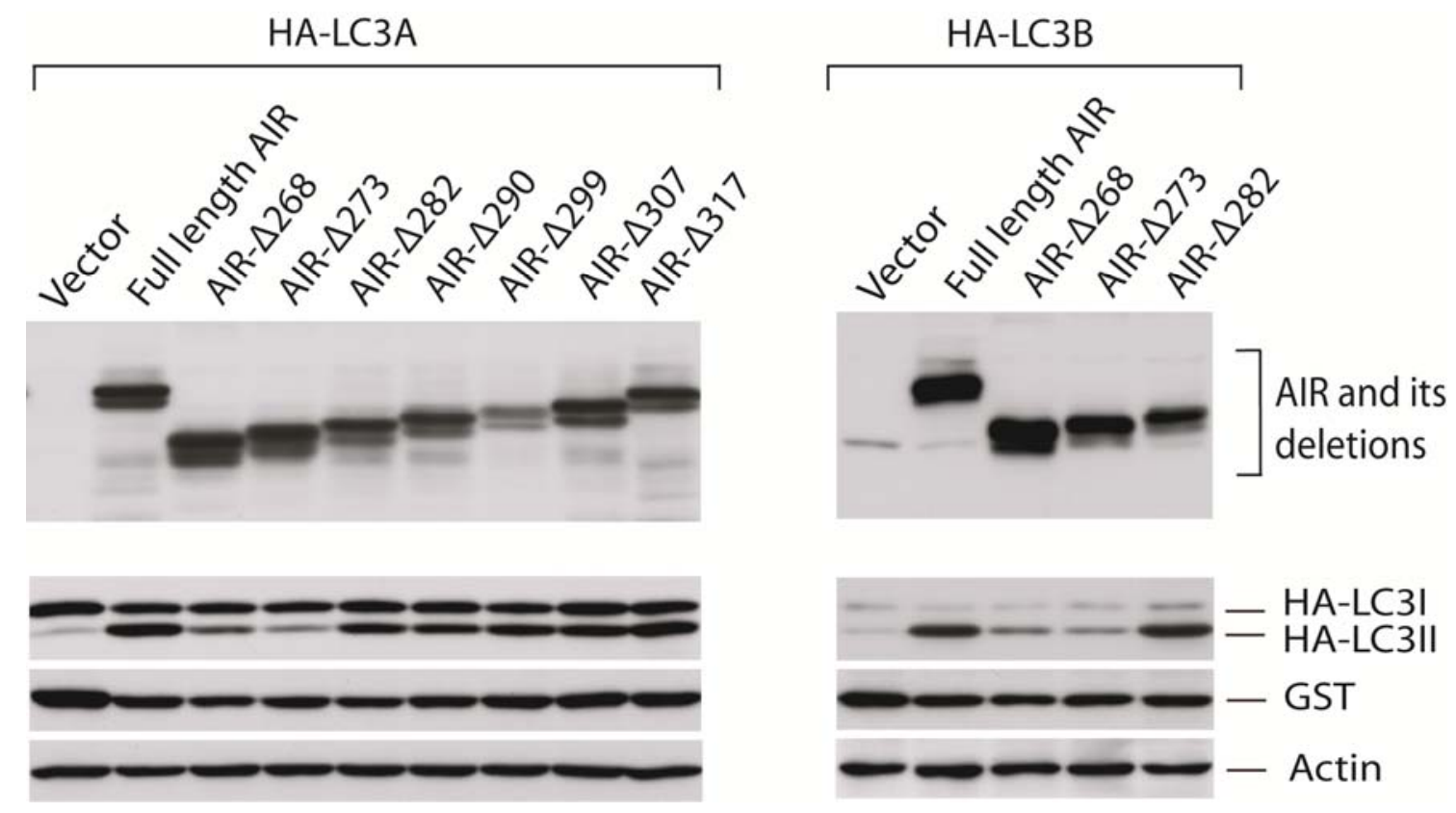

Figure 18: C-terminal deletions of AIR remain active up to AIR- $\mathbf{2} 282$.

HEK-293 cells were transfected with the indicated plasmids and HA-LC3A or HA-LC3B together with GST as a transfection control. 40h post transfection the cells were lysed and prepared for Western blotting. The deletions remain active up to the $\Delta 282$; greater deletions do not induce lipidation of the co-transfected autophagy marker LC3 when compared to the full length construct. WB anti-AIR shows that all constructs are expressed well. WB anti-HA indicates activation of LC3. WB anti-GST demonstrates that the differences in LC3II are not due to uneven transfection levels, and the WB anti-Actin proves equal loading. 


\section{The intracellular domain of AIR is sufficient to induce autophagy}

The next step was to find out whether the intracellular part of AIR on its own was sufficient to induce autophagy. To do this, we placed the intracellular domain of AIR in a completely different molecular background: chimeric membrane molecules known to be expressed at the cell surface (CD16:7). The CD16:7chimera consists of the extracellular part of the CD16 receptor (including a leader region that directs it to the cell surface) and the transmembrane domain of the T-cell antigen CD7. This kind of hybrid molecules offers the possibility to be stimulated with specific anti-CD16 antibodies that act as artificial ligands by promoting aggregation. The empty chimera CD16:7 lacking any part from AIR was used as a negative control in these experiments (figure 19A). After co-transfection of HEK-293 cells with the relevant chimeric constructs, the autophagy reporter HA-LC3A and the transfection control GST, cells were stimulated with the anti-CD16 antibody at two different concentrations. Comparing the samples in the Western blot there is a clear increase of the LC3II band in the samples transfected with CD16:7:AIR-ID that also depends on the concentration of the artificial ligand. In contrast, there is a hardly visible band in the samples transfected with the empty chimera and this band fails to increase on addition of the anti-CD16 antibody (figure 19B). Therefore, autophagy induction in this system depends only on the intracellular part of AIR, thus indicating that this domain is not only necessary but also sufficient to induce autophagy.

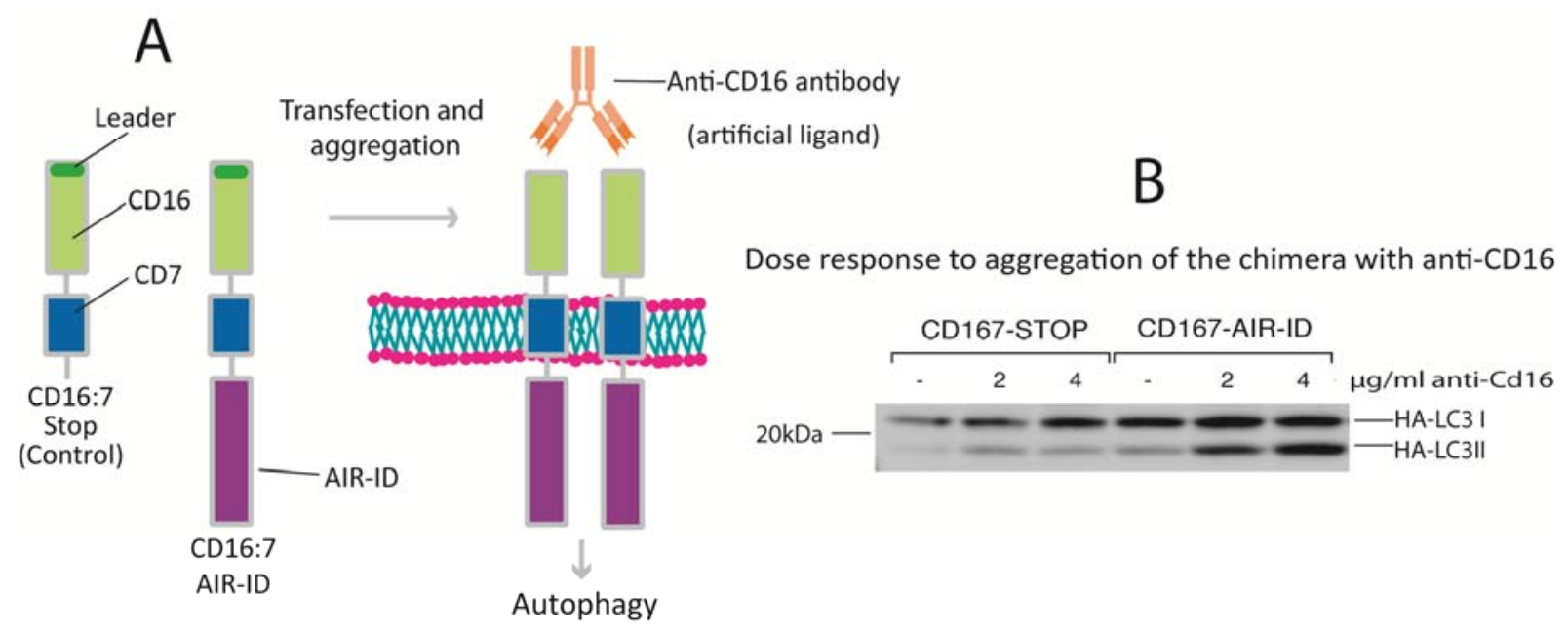

Figure 19: The chimera CD16:7:AIR-ID can be stimulated by its artificial ligand.

A Schematic description of the chimera CD16:7 which consists of a leader sequence, the extracellular part of CD16, the transmembrane part of CD7 and the intracellular part of AIR. In the following experiments the empty chimera which is also called CD16:7 Stop is used as a negative control since it lacks any part of AIR. Stimulation of the chimera with the artificial ligand, an antibody against CD16 leads to aggregation of the 
artificial receptor and subsequently autophagy induction as can be seen in B CD16:7-AIR-ID stimulation by anti-CD16 is concentration-dependent. HEK-293 were transfected with HA-LC3A and put into a 0 (control), 2 or $4 \mathrm{mg} / \mathrm{ml}$ anti-CD16 containing growth medium $40 \mathrm{~h}$ post-transfection. Further $24 \mathrm{~h}$ later the cells were lysed and prepared for anti-HA Western blotting. The blot shows that CD16:7 Stop does not provoke LC3II formation even when incubated with the artificial ligand. It also demonstrates that aggregation of CD16:7AIR-ID with the anti-CD16 antibody induces autophagy and that the extent of the LC3 lipidation depends on the antibody concentration.

\subsection{The active subdomain of AIR is sufficient to induce autophagy}

As we had already explored the autophagy-inducing properties of AIR deletions, we set out to apply these findings to the chimera experiments. We fused CD16:7 with the intracellular part of the two C-terminal deletions that remained active in the overexpression experiments (see figure 18, AIR $\Delta 290$ and AIR $\Delta 282$ ). To gain insight into whether some of the amino acids located at the $\mathrm{N}$-terminus of the intracellular protein were necessary for autophagy induction, we also introduced N-terminal deletions of AIR-ID into the context of the CD16:7 chimera. As additional controls two chimeras containing the inactive parts of the intracellular domain of AIR were also generated. The new chimeras which were subsequently tested in aggregation experiments were the C-terminal deletions CD16:7AIR-IDAR290 (=CD16:7-AIR-263-289) and CD16:7-AIR-IDAF282 (=CD16:7-AIR-263281), the N-terminal deletions CD16:7-AIR-Y268-323, CD16:7-AIR-F282-323 and CD16:7-AIR-R290-323, as well as the $\mathrm{N}^{-}$and C-terminal deletions CD16:7-AIR-

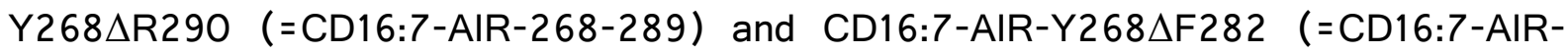
268-281). CD16:7-AIR-R290-323 and CD16:7-AIR-F282-323 carry the intracellular part of AIR which is missing in CD16:7-AIR-ID $\Delta$ R290 and CD16:7-AIR-ID $\Delta F 282$, respectively (figure 20).

The intracellular domain (ID) of AIR and the deletions which were cloned into CD16:7

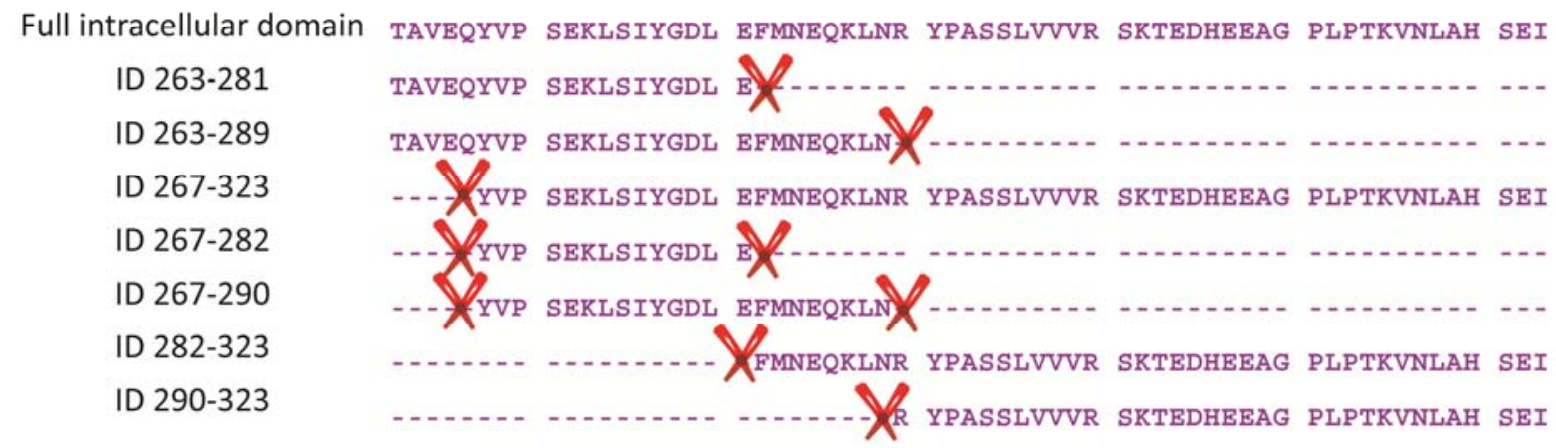

Figure 20: Intracellular AIR domain as part of the chimera.

The scissors indicate where the sequence was cut off in the different deletions. The lower two sequences represent the deleted parts in the first two deletions. The middle three sequences are the corresponding $\mathrm{N}$ terminal deletions for the first three sequences. 


\subsection{Expression levels of the different chimeras on the cell surface}

To be able to compare the results obtained with the different chimeric constructs, it was important to know whether they were expressed at similar levels on the cell surface. For this purpose, the different chimeras were transfected into HEK-293 cells and subsequently stained with a FITC-coupled antibody against CD16. The FITC signal was measured by flow cytometry and the mean fluorescence shown by the cells transfected with the different constructs was compared. The cells with the empty chimera had a slightly lower fluorescence mean than the cells with CD16:7-AIR-ID. The chimeras CD16:7-AIR-Y268-323 and CD16:7-AIR-Y268AF282 were less abundant on the cell surface than the empty chimera or the chimera containing the full ID. Surprisingly, the Cterminal deletions $\Delta \mathrm{R} 290$ showed extremely high fluorescence means, and therefore much higher expression rates than the other chimeras at the cell surface (figure 21B). As mentioned above, the intracellular AIR domain contains several motifs involved in endocytosis and deletion of these could lead to less endocytosis and subsequently more chimera on the cell surface. However, this possibility does not explain the very high expression levels of the $\triangle R 290$ deletion, because it retains the $Y X X L$ motif from aa 277281. Looking at the motifs which have been removed in $\triangle R 290$ there is an EXXXXL dileucine-like motif (aa 308-313) that is involved in endosomal sorting, but the same happens with the $\Delta \mathrm{F} 282$ deletion, which shows lower expression than the full length intracellular domain. This finding suggests that the dileucine-like signalling motif does not greatly influence the abundance of these constructs on the cell surface. The possibility that missing DXEs, export-from-ER-signal-motifs are responsible for the inhomogeneous presence on the cell membrane is also unlikely. Although $\Delta \mathrm{R} 290$ carries two of these motifs and $\Delta \mathrm{F} 282$ only one, a fact which could theoretically cause lower expression of the latter, the chimera with the full length ID and both DXEs still shows considerably lower expression levels on the cell surface compared to $\Delta \mathrm{R} 290$.

Despite the possibility that these motifs could influence the presence of the different constructs on the cell surface to some extent, they cannot completely explain the different expression levels detected. Hence we need to acknowledge that further studies are required in order to find out what causes the differences. However, in the subsequent functional evaluation of these constructs we need to consider the varying quantities of chimeras on the cell membrane when comparing the changes they induce in the autophagic marker, since a more active construct could simply mean a higher membrane expression level. Following this rationale, the degree of autophagy caused by chimeras 
with lower cell surface expression than CD16:7-full-AIR-ID is probably underestimated, which is beneficial for the relevancy of the result.
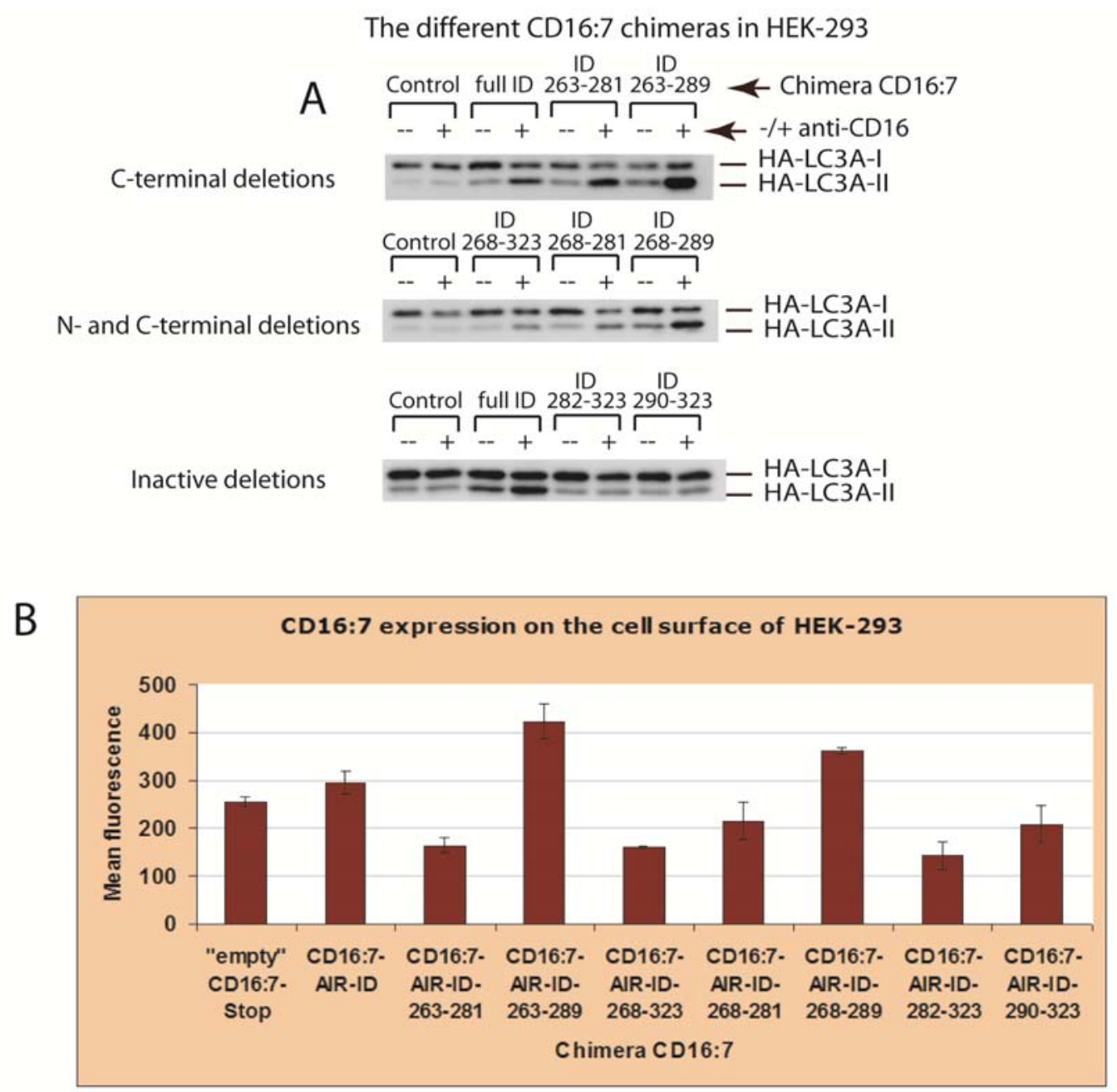

Figure 21: The chimera with the biggest active deletion of AIR-ID CD16:7:AIR-ID-263-281 is expressed at the cell surface at levels comparable to CD16:7:AIR-full-ID.

A $C D$ 16:7:AIR-ID-263-281 is the chimera with the biggest deletion of AIR-ID retaining its full capacity as an LC3// inducer. HEK-293 were co-transfected with plasmids expressing the different chimeras, HA-LC3A and GST as a transfection control (not shown). After 24h the cells were split into two wells, for aggregation with the artificial ligand, the antibody CD16, which took place overnight later. After a further $24 \mathrm{~h}$ the cells were lysed, the protein levels were measured and the samples were prepared for Western blotting, so that the Western blots show the samples with and without antibody addition $24 \mathrm{~h}$ after aggregation as indicated. B The $\Delta 290$ deletions are expressed above average and greater deletions are slightly underrepresented on the cell surface. Cells were transfected in duplicate and their surface was stained with a FITC-coupled antiCD16 antibody 40h later. The cells were kept in sodium azide at all times during the staining and measured with the cytometer (numbers based on 10000 counted cells per experimental point). The graph shows the mean fluorescence of the stained cells recorded by cytometry in two experiments which were both carried out in duplicate. The bars show the standard error.

\subsection{Active and inactive CD16:7-AIR chimeras}

Both C-terminal deletions of the intracellular domain (CD16:7-AIR-263-289 and CD16:7AIR-263-281) are fully active and inducible by antibody stimulation, whilst the $\mathrm{N}$-terminal 
deletions that represent the missing part of these mutations do not show an increase of the LC3II band in anti-HA Western blots from transfected HEK-293 cells (figure 21A). $\triangle R 290$ seems even more active than the full length ID chimera, although this is probably due to the higher expression levels as detected by flow cytometry (see figure 21B). The $\mathrm{N}$-terminal deletion CD16:7-AIR-Y268-323 and the corresponding $\mathrm{N}$ - and C-terminal deletion CD16:7-AIR-Y268 $\mathrm{F} 282$ show decreased activity. The induction rate of autophagy in the case of CD16:7-Y268 R290 is probably caused by the high expression level of a partially active construct. An increased expression level is also likely to have caused the strong activation of LC3 by CD16:7-AIR-ID $\Delta$ R290. Thus, we stopped working with the $\Delta R 290$-deletions because of their extremely high expression on the cell surface in comparison with the full ID.

CD16:7-AIR-ID- $\triangle F 282$ was shown to be the shortest amino acid sequence of AIR capable of inducing autophagy at levels comparable with the activity triggered by the full intracellular part (figure 22). Therefore, by means of these studies we have reduced the pro-autophagic domain of AIR to a short 19 amino acid stretch located right after the membrane region within the intracellular domain of the molecule.
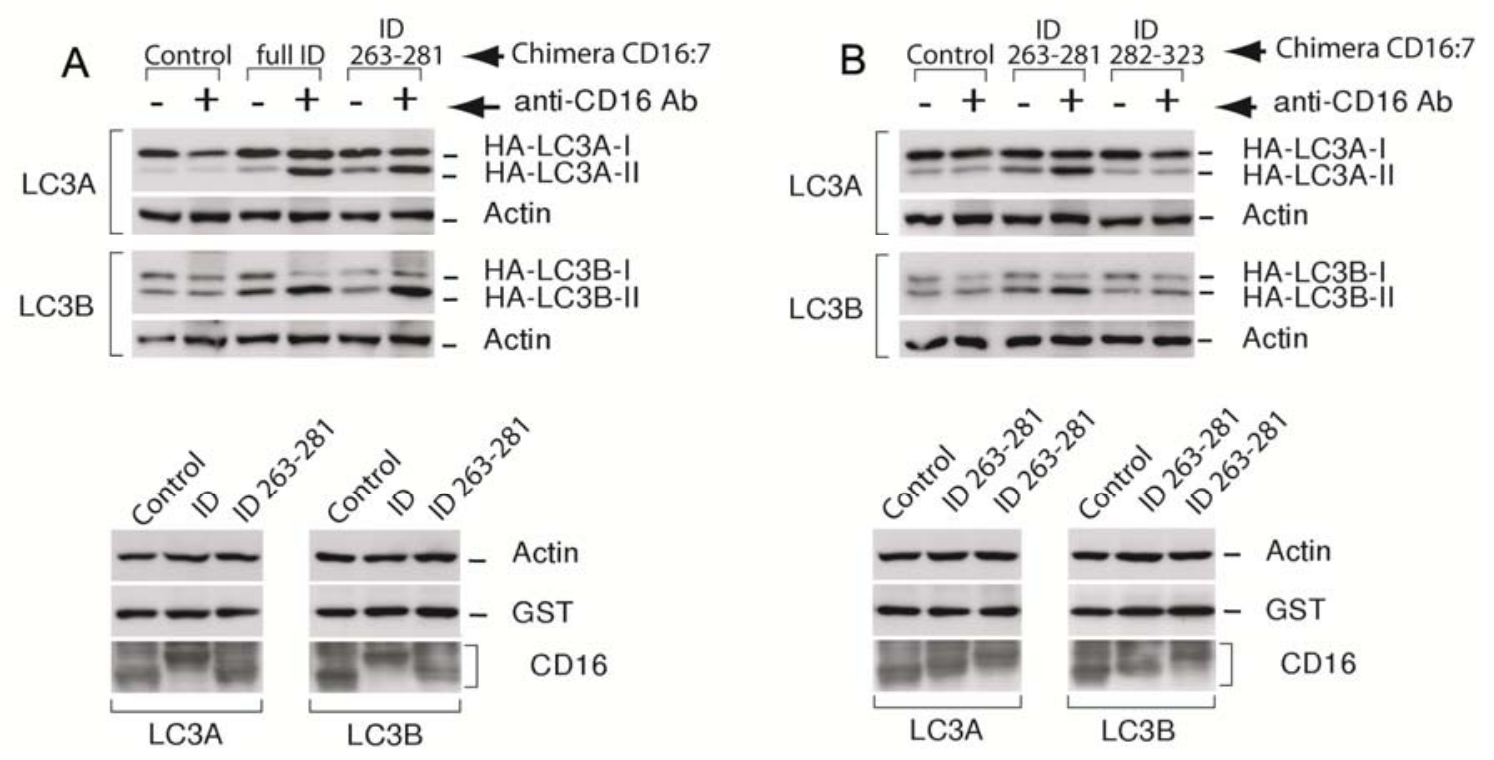

Figure 22: The chimera CD16:7:AIR-ID-263-281 contains the active subdomain of AIR.

A CD 16:7:AIR-ID-263-281 excites autophagy as strongly as CD16:7 AIR-full-ID. B The corresponding "missing part" of CD 16:7:AIR-ID-263-281 is inactive. The experimental design is the same as in figure $21 \mathrm{~A}$ only that the experiment was carried out for LC3A and LC3B. The controls below show only the experimental point without antibody addition as $-/+$ aggregation originates from the same transfection, and the CD16 antibody interferes with the immunological reactions in the subsequent antibody incubations. 


\section{AIR and its subjection to the autophagic machinery}

Having identified the chimera with the greatest active deletion, we wanted to know whether the LC3 lipidation depended on the general mechanisms involved in macroautophagy or if, on the contrary, AIR signals via an alternative and possibly unknown autophagic pathway. For this purpose we first chose a pharmacological approach and investigated the mechanisms (or molecular circuitry) of AIR-induced autophagy in additional experiments based on RNA silencing of Atg expression.

\subsection{AlR and autophagy inhibiting drugs.}

As mentioned in the introduction, the class III PI3-kinase Vps34 is required as part of a protein complex needed for autophagosome formation, and can be negatively regulated by PI3-kinase inhibitors such as Wortmannin (WM) or 3-Methyladenine (3-MA).

In the aggregation experiments with the transfected chimeras CD16:7-AIR-ID- $\Delta F 282$ (containing the 19 amino acid active subdomain) and CD16:7-AIR-ID, the activation of the co-transfected HA-LC3A was reduced with either of the PI3-kinase inhibitors. While the cells treated with normal medium showed substantial amounts of lipidated LC3, cells treated with 3-MA or WM had very little LC3II (figure 23). As these drugs suppress a critical step in the canonical autophagic pathway, this result suggests that AIR induces conventional macroautophagy.

The chimeras in HEK-293 cells after treatment with PI3K-inhibitors
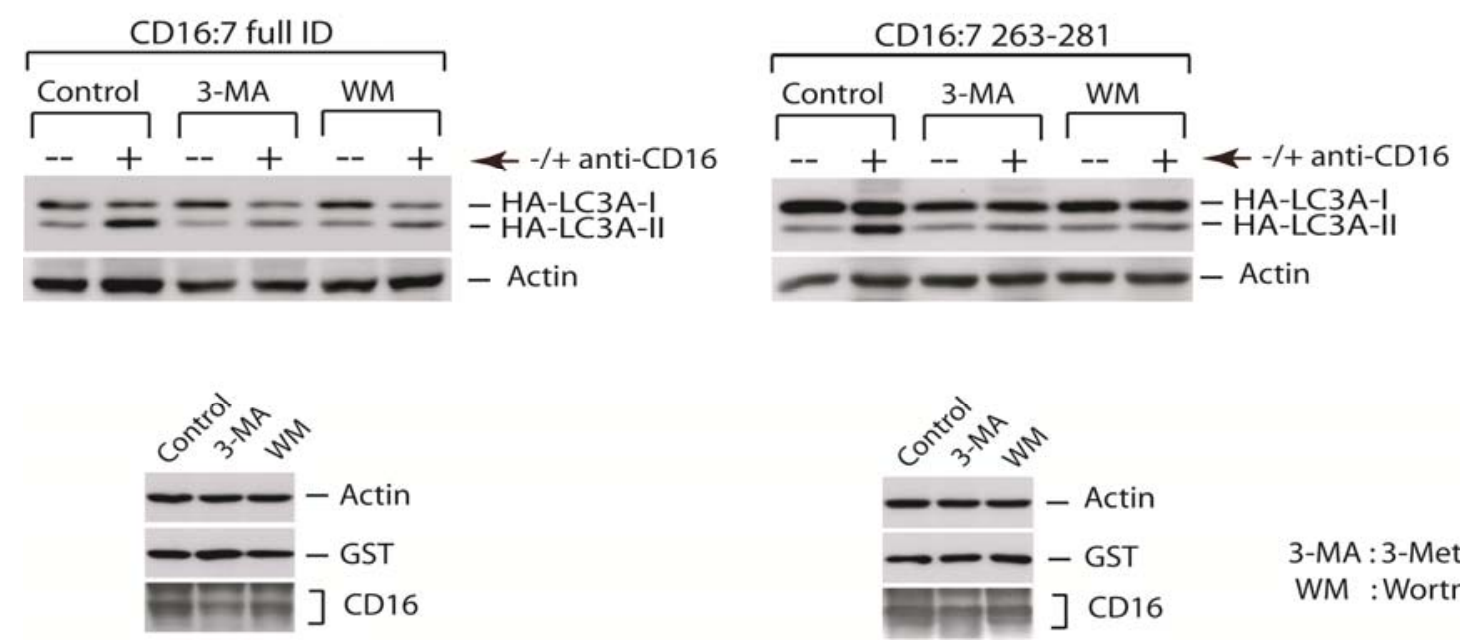

3-MA : 3-Methyladenine WM :Wortmannin

Figure 23: The phosphoinositide 3-kinase inhibitors 3-Methyladenine (3-MA) and Wortmannin (WM) reduce AIR-induced autophagy.

HEK-293 were co-transfected with plasmids expressing the indicated chimeras, HA-LC3 and GST as described in figure 21A. 30 min before treatment with the artificial ligand anti-CD16, the PI3K inhibitors were added to give a final concentration of $0.2 \mu \mathrm{M}$ WM or $10 \mathrm{mM} 3-\mathrm{MA}$. To the samples treated with WM, the 
same amount of the drug was re-applied 12h later. In both chimeras tested, CD16:7 full ID and CD16:7 263-281, the PI3K inhibitors led to less lipidation of LC3 while the controls show equal transfection and loading. As explained above, the controls show only the samples which have not been treated with the antiCD16 antibody as the cell lysates with and without aggregation originate from the same transfection.

\subsection{Silencing of important proteins in the macroautophagy pathway}

After having shown that the type of autophagy induced by AIR depended on the PI3kinase, Vps34, a central component of the macroautophagy signalling pathway, we wanted to find out whether the novel protein also depended on other key proteins involved in this pathway. Additionally, we wanted to investigate the involvement of AIR in the conventional autophagy signalling pathway not only by using pharmacological agents but also with a genetic approach. Therefore, we knocked down the central autophagy related proteins Atg5, Atg7 and Beclin1 using small inhibiting RNAs. Atg5 and Atg7 play a key role in LC3 lipidation (see introduction section 5.3.2. and figure 3). Beclin1 is a component of the Vps34 protein complex (see introduction 5.3.1. and figure 3). The Beclin-Vps34 complex also mediates formation of the autophagosome, although it does so at an earlier step than Atg5 and Atg7.

In order to find out whether AIR-induced autophagy depended on the presence of Atg5, Atg7 and Beclin1, we knocked down each of these proteins in HEK-293 cells and subsequently carried out the chimera experiment as usual by transfecting the chimera followed by aggregating it with its artificial ligand, the anti-CD16 antibody.

As can be seen in the control Western blots shown in figure 24 , there is a considerable expression level decrease of all three autophagy related proteins in HEK-293 cells transfected with the corresponding siRNAs. Concomitantly, autophagy induced by the CD16:7-AIR-ID and CD16:7-AIR-ID- $A F 282$ chimeras was clearly reduced in the absence of Atg5 and Atg7 as can be deduced from comparing the LC3II levels between the relevant points in WB. On the contrary, Beclin1 knockdown had no effect on LC3 lipidation. Therefore, these experiments show that the autophagic process stimulated by AIR is indeed mediated by the canonical mediators Atg5 and Atg7, but it is also somewhat unconventional in the sense that it seems to proceed in a Beclin1-independent manner. 

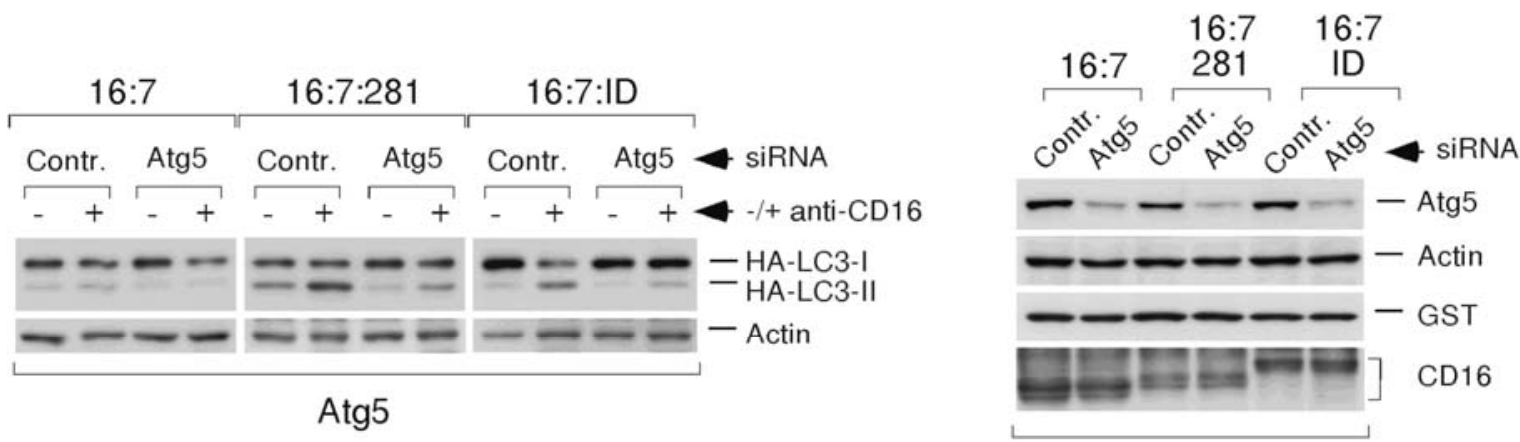

Unaggregated samples

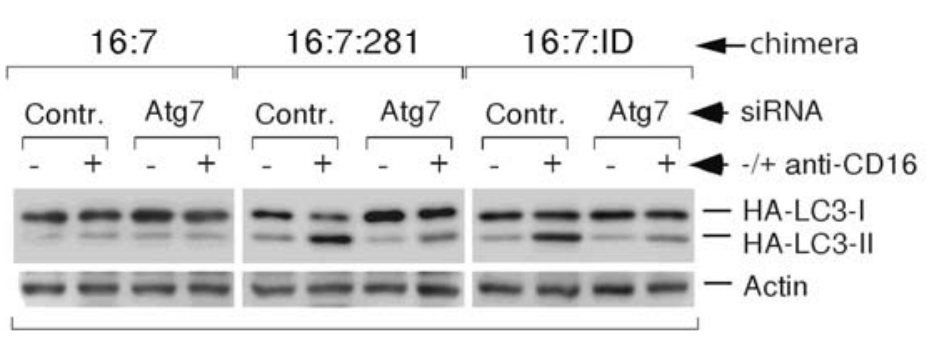

Atg7

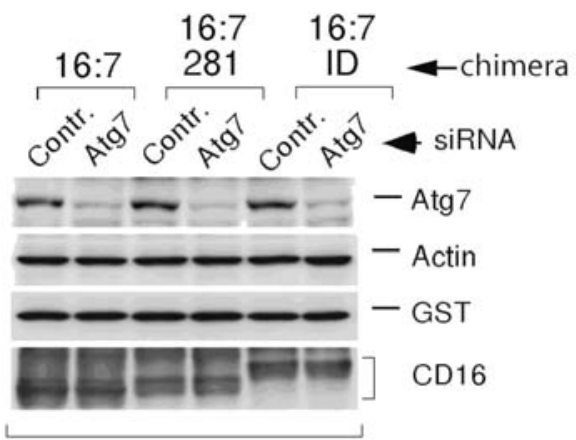

Unaggregated samples
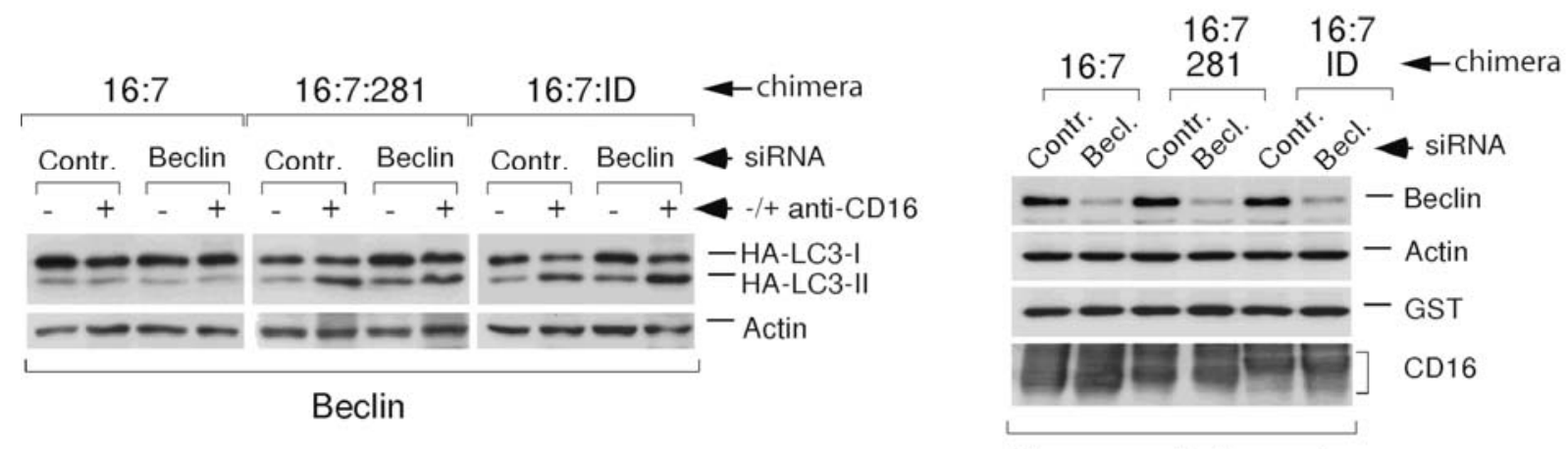

Unaggregated samples

Figure 24: AIR-induced LC3II formation depends on Atg5 and Atg7 but not on Beclin1.

HEK-293 were transfected with siRNAs against Atg5, Atg7, Beclin1 (or with siRNA anti-control) and 11h later the cells were transfected again with plasmids expressing the indicated chimeras, HA-LC3A and GST. The same experimental procedure as described in the previous chimera experiments, including splitting of the cells and antibody addition followed. The Western blots show depletion for all three silenced proteins, while the loading and transfection controls demonstrate homogeneity. In the case of Atg5 and Atg7 the knock-down causes less lipidation of the transfected LC3 in CD16:7-AIR-ID and CD16:7-AIR-263-281. In the samples with Beclin1 depletion there is no detectable effect on the levels of LC3II. 



\section{Discussion}

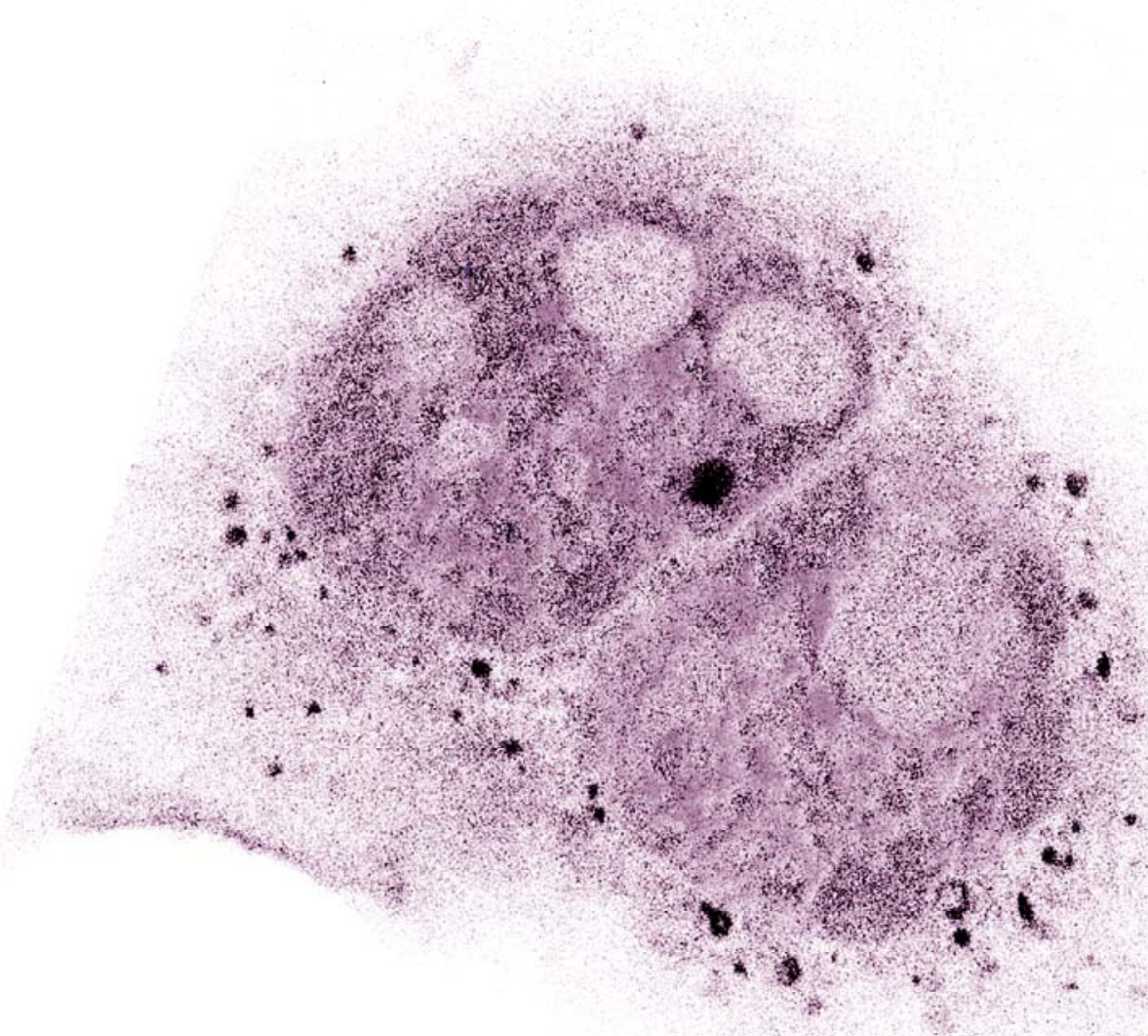





\section{The previously uncharacterised protein AIR is a general inducer of autophagy}

As was described in the results chapter, the clone 9-1.4.-P15 previously identified from a high throughput screening (Alcala et al. 2008) provokes autophagy when overexpressed. This finding could be reproduced in various cell types, including cells with low expression of the transfected plasmid, thus proving its effect as an autophagy inducer to be neither cell- nor tissue-specific.

\section{Overexpression of proteins as a strategy for functional studies}

It is known that overexpression of many proteins activates the signalling pathways downstream of the ectopically (or pathologically, see oncogenes) expressed protein. This finding has been used before to discover, for example, apoptosis inducers (Albayrak \& Grimm 2003), activators of the NFKB pathway (Matsuda et al. 2003), effectors of the transcription factor activating protein 1 (Chanda et al. 2003) or modifiers of APP shedding (Schobel et al. 2006).

\subsection{Overexpression of AIR induces non-apoptotic cell death with autophagic features}

Coming from a screening for cell death inducers, the newly identified protein led to a nonapoptotic type of cell death. A very similar type of cell death had been previously reported for Bak/Bax deficient MEFs that died in a macroautophagy-dependent manner. A caspase-independent but Atg5- and Beclin1-dependent type of cell death occurred in these cells upon etoposide treatment (Shimizu et al. 2004). Similar to the effect of AIR in HEK-293T cells, these dying Bak/Bax deficient MEFs became rounded and showed a ballooned morphology. A further feature of this death process also shared by the cell loss following transfection of 9-1.4.-P15 was caspase-independence. Additionally, autophagosome formation and LC3 activation took place in the dying Bak/Bax deficient MEFs as well as in HEK-293T transfected with the clone 9-1.4.-P15. Taken together, these observations strongly imply that AIR is capable of inducing the same death type as found by Shimizu et al. (figure 10). 


\section{AIR is a strong inducer of LC3 lipidation}

As described in the introduction (see 5.3.1.), LC3 is the most widely used autophagic marker. Its conversion from cytosolic LC3I to the membrane-bound form LC3II makes the formation of autophagsomes detectable by Western blotting and fluorescence microscopy. An advantage of using tagged versions of LC3 for both techniques (immunoblotting and microscopy) is that only transfected cells are analysed. In order to have a valid positive control we used a homologue of BNIP3, (BNIP3L) described by Kanzawa et al. as an autophagic cell death inducer (Kanzawa et al. 2005). Both homologues induce apoptosis if not suppressed, which is why we inhibited apoptosis in our experiments with BNIP3L by co-transfecting an expression plasmid harbouring the viral caspase inhibitor p35. Surprisingly, AIR induces autophagy even more strongly than its positive control. Judging by the amount of lipidated LC3, AIR shows more LC3II than BNIP3L in both of the LC3 isoforms $(A / B)$ studied. In most cases, when comparing AIR with the empty vector or BNIP3L, the samples transfected with AIR also show less LC3I. This result is plausible as LC3II originates from LC3I and thus confirms AIR as an even stronger autophagy inducer than BNIP3L. In fluorescence microscopy, quantification of GFP-LC3A dots shows slightly more punctae in AIR than in BNIP3L, a difference which increases when looking at HEK293 co-transfected with the B isoform of GFP-LC3 (figures $11 \& 13$ ).

\section{Evolutionary conservation of AIR}

Many of the proteins implicated in autophagy are conserved from yeast to humans, but our novel protein has no known homologue in yeast (figure 12). This could mean that AIR is not implicated in macroautophagy as a starvation response but rather in one of the autophagy-related processes which play a role in immunity or neurodegeneration and which do not exist in yeast. There are examples of yeast autophagy-related proteins that have gained functions related to immunity in higher eukaryotes, for example Atg16L. The mammalian Atg16L1 protein is four times as long as its yeast homologue Atg16. Within the additional sequence there are $7 \mathrm{C}$-terminal WD40 repeats (the WD domain is named after tryptophan and aspartic acid in which these 40 amino acid peptides often terminate). The immunologically relevant Crohn's disease related SNP T300A is to be found in this part which is absent in the yeast homologue (Hampe et al. 2007), pointing to the notion that this additional autophagy function in immunology is accompanied by extra domains or even proteins. Therefore, it would not be surprising that, although autophagy is an evolutionarily 
conserved process, there are autophagy inducers that have no homologue in yeast. The fact that our novel protein is conserved from fish to humans could indicate that AIR has a role in autophagic processes that are particularly relevant for vertebrates like immunity, phagocytosis or neuroprotection, as these processes are biological functions which gain importance in higher organisms. This hypothesis could also explain a result we obtained from preliminary AIR knock-down experiments in SAOS-2 and HeLa cells. Transfecting these cells with siRNA against AIR had no significant impact on their starvation response measured by means of LC3 (data not shown).

\section{Low expression levels of AIR}

An important step towards unravelling the function and whereabouts of AIR was to produce an antibody able to detect endogenous AIR. However, detection of endogenous AIR was difficult to achieve. This could be due to low protein levels in the cell, caused by either little protein synthesis or rapid degradation. Problems with the antibody sensitivity are also possible, although we were able to detect endogenous AIR in Western blots after immunoprecipitation. Ullrich et al, in their paper on AIR published while we were characterising it (Ullrich et al. 2010), could not achieve any type of AIR detection other than by immunofluorescence, suggesting that low protein levels rather than poor antibody quality might cause these difficulties when detecting AIR.

\subsection{AIR localises to the endosomal-lysosomal compartments}

Ullrich et al. found AIR to be mainly expressed at the Golgi-apparatus. However, in our studies AIR hardly showed any colocalisation with the Golgi marker GM130 also used by Ullrich et al. Instead we found AIR to be mainly expressed in the endosomal or lysosomal compartments by means of endosome and lysosome markers CD63 and LAMP2. Although this colocalisation is clear, it is not complete. Both AIR and the markers show a few punctae which do not coincide in the merged images of AIR (green) with either CD63 or LAMP2 (both red). Assuming similar antibody quality this means that AIR is also found in other compartments and that it is not simply a lysosomal or endosomal protein. The finding that there seem to be more marker punctae than AIR punctae could also indicate that AIR is in a subpopulation of the endosomal-lysosomal compartments. The observation of Ullrich et al. that AIR is in the Golgi-apparatus is probably due to its mere passage through the Golgi-apparatus. Moreover, while we used antibodies generated against the $\mathrm{N}$ terminus of AIR, Ullrich et al. used an antibody that detects the C-terminus of the protein. Therefore, we cannot rule out that different antibodies detect distinct pools of AIR that, for 
example, could be emerging through cleavage events. Nevertheless, based on the comparison of AIR double stainings with three different cell compartment markers we deduce that AIR is mainly localised in the endosomal-lysosomal compartments of the cell (figure 15).

\subsection{AIR is transiently located on the plasma membrane but is rapidly endocytosed}

Having found our novel protein in endosomal compartments and expecting it to be a membrane receptor, the next step was to reproduce what already had been achieved with transfected AIR: its detection on the cell surface. While this was impossible in untreated cells, it also could not be accomplished after inhibiting endocytosis, for example by cotransfecting non-functional dynamin mutants. The only endocytosis inhibitor which was capable of making AIR appear on the cell surface in immunofluorescences was chloroquine (figure 16).

The pharmacological agent chloroquine inhibits the acidification of the late endosomes and was found to inhibit cycling of the membrane protein, LAMP1, from the plasma membrane surface to the lysosome (to where it predominantly localises) and back to the cell surface. Chloroquine blocks the trafficking of LAMP1 from the endosomes to the lysosome, thus resulting in higher levels of LAMP1 in both the endosomes and the cell surface (LippincottSchwartz \& Fambrough 1987). In this publication, the cycling of LAMP1 is compared to the trafficking of a receptor which binds to its ligand on the cell surface, dissociates from it in the lysosome and is recycled when returning to the cell surface. In view of our results from the localisation studies for AIR, it seems likely that our novel autophagy inducer is rapidly endocytosed and efficiently targeted to the lysosome where it is either degraded or recycled in a similar manner to the cycling of LAMP1 or subjected to both scenarios.

\subsubsection{Endocytosis contributes to autophagosome formation.}

Lately, it has been shown that the importance of endocytosis for autophagy is greater than previously thought. Apart from the well established finding that endocytic vesicles fuse with autophagosomes (see Introduction section 6.), it seems that pre-autophagosomal structures can originate from the plasma membrane. Thus, in a recently published study, phagophores were shown to form through endocytic scission of the plasma membrane as ATG16L interacted with the coating protein from the endocytic vesicles, clathrin. Further it was demonstrated that the formation of autophagosomes and their precursors is impaired when inhibiting clathrin-mediated endocytosis (Ravikumar et al. 2010). Until now, the 
origin of the autophagosomes remains unclear and this characteristic double membrane is thought to be formed de-novo (see Introduction section 5.3.), possibly with the mitochondria or the endoplasmatic reticulum participating in the process of membrane formation.

In the light of AIR being constantly endocytosed, the case of the pre-autophagosomal structures forming from the plasma membrane appears especially interesting as this raises the question whether AIR could be present in these autophagosome-initiating complexes. A possible way to investigate this could be confocal colocalisation studies with AIR, clathrin and Atg16 or co-immunoprecipitation studies with the proteins of the Atg-machinery that usually surround Atg16 as part of a complex, Atg12 and Atg5 (see Introduction section 5.3.). The hypothetical interaction of AIR with the Atg16L-Atg5-Atg12 complex could also have an impact beyond the labelling of autophagosomes that arise. While in the study by Ravikumar et al., recruitment of Atg16 $\mathrm{L}$ to the plasma membrane and endocytosis were important for seemingly standard starvation-induced autophagy, Atg16L localisation to this site is also important for autophagic degradation of bacterial invaders. When targeting pathogens for elimination through autophagy, Atg16L recruitment to the plasma membrane is directed by Nods (see Introduction section 5.3.2. and 8.2.1 (Travassos et al. 2010)), thereby linking this protein to Nod-mediated autophagy against bacterial invaders. Various polymorphisms in Atg16 L have been associated with the autoimmune disease, Crohn's disease, as a probable consequence of defective immune defence. Thus, if some type of interplay between AIR and the autophagic machinery implicated in autophagosome formation were to be found, this would suggest a possible implication of AIR in autophagy related to innate immunity and pathogen clearance.

\section{The intracellular part of AIR is necessary for autophagy induction}

Based on the amino acid sequence of AIR showing an $\mathrm{N}$-terminal leader sequence, we assumed that the part of AIR following this leader was extracellular, and that the Cterminal part of AIR thus should be intracellular. Additionally, this type 1 topology is backed up by the immunofluorescence staining of the cell surface as the AIR antibody was generated against the $\mathrm{N}$-terminus of the protein fused to GST. A positive surface staining of the unpermeabilised cell with this antibody therefore shows that the N-terminal of AIR lies outside the cell, as it could be recognised without permeabilising the cell first. By deleting the intracellular part of AIR and expressing this mutant in HEK-293 cells together 
with the autophagic marker, LC3, it could be shown that the intracellular part of the autophagy inducer is necessary for its effect on LC3 (figure 17).

\subsection{AIRU 282 remains fully functional as an autophagy inducer}

By employing smaller deletions of the intracellular domain than $\triangle I D$ in subsequent experiments, we were able to narrow down the necessary domain for autophagy induction. AIR $\Delta 282$ was still able to induce autophagy which implies that the amino acids $282-323$ are not necessary for autophagy signalling (figure 18).

\subsection{AIR could function as a transmembrane receptor}

A necessary intracellular domain is consistent with the idea of the extracellular part binding a putative ligand and the intracellular part signalling to the downstream pathway to induce autophagy. Unfortunately, this hypothetical AIR ligand is unknown and therefore cannot be used for activation and further studies of the autophagy-inducing domain. An artificial ligand, such as the AIR antibody, which could be added to the cell medium, is also unsuitable in view of the fact that AIR seems to be rapidly endocytosed and transported to the endosomal-lysosomal compartments, thus leading to a minimal presence of the protein on the cell surface.

\section{The intracellular AIR domain is sufficient for autophagy induction}

As part of a receptor, one would expect the intracellular part of AIR to be capable of inducing autophagy independently of the extracellular AIR domain, if activated accordingly. Hence, the intracellular part was placed in the adequate cell compartment and was stimulated. Adequate placement and stimulation with an artificial ligand could be achieved by using the chimera CD16:7. Once cloned into this new molecular context, we could prove that the intracellular domain of AIR was sufficient to induce autophagy. In a small dose response experiment it was shown that the CD16:7 chimera and the anti-CD16 antibody work as a receptor-ligand system; treatment of the chimera with two different antibody concentrations demonstrated that the activation of the receptor (CD16:7) in this context depended on the concentration of the ligand (anti-CD16 antibody) (see figure 19B). 


\subsection{Structural and functional studies using CD16:7-AIR chimeras}

The CD16:7-AIR chimera constituted a practical tool for the structure-function studies of AIR because by using the "empty" CD16:7 chimera we had an ideal negative control. This molecule, on the one hand localised to the cell membrane and could be aggregated with the antibody just like the to-be-studied construct, but on the other hand it neither expressed a single part from AIR, nor did it induce autophagy. Thereby it could be ensured that the differences in results from the empty chimera and the to-be-studied construct were entirely due to the AIR part cloned into the chimera.

\subsubsection{Comparable surface expression of the relevant CD16:7 AIR chimeras}

Different chimera constructs might be leading to different expression levels on the cell surface, owing to different endocytosis rates or other incidents. Lower presence on the cell surface could compromise the activity as an autophagy inducer and vice versa. Thus, the possibility of confusing higher expression on the cell surface with higher autophagic activity had to be ruled out. This was done by using flow cytometry (figure 21B). Chimera constructs with over-average expression on the cell surface were not included in further experiments and the chimera carrying the active subdomain used in subsequent trials shows even lower expression on the cell surface than CD16:7:AIR-full ID. Although this circumstance initially seems counterproductive for our purpose, the pro-autophagic activity shown by these constructs is even more valid as it is produced by a molecule with a lower-than-average presence on the cell surface.

\section{AIR-263-281 is the shortest amino acid sequence of AIR- ID fully capable of inducing autophagy}

Experiments with C-terminal deletions of the entire AIR protein had already hinted that amino acids 282-323 were unnecessary for the induction of autophagy, and accordingly constructed CD16:7 chimeras carrying C-terminal deletions of the intracellular AIR part led to the same conclusions. In order to map the active subdomain of AIR, N-terminal deletions of the intracellular domain were also cloned into the chimera CD16:7. Experiments with these hybrids demonstrated that even a small $\mathrm{N}$-terminal deletion of five amino acids derogated the autophagy inducing properties of the construct, and removing eleven amino acids from the $\mathrm{N}$-terminal of the intracellular domain caused little to no 
activity. In this context it was not surprising to find that after deleting $19 \mathrm{~N}$-terminal amino acids (which form the remaining amino acid sequence of the biggest active C-terminal deletion cloned into CD16:7-AIR-263-282) no autophagic activity at all could be measured by Western blot. Thus, by means of the chimera it was possible to narrow the active subdomain of AIR down to 19 amino acids.

\section{The active subdomain of AIR contains an endocytosis motif and is likely to be phosphorylated}

Within the 19 amino acids of the active AIR subdomain there is a YXXL motif from amino acids 277-281. This sequence is a recognised signal for endocytosis and trafficking of receptors, and is also important for lysosomal targeting. This type of motif as a crucial part of the signalling domain is in line with what we have previously found out about AIR. According to this, AIR is transiently expressed at the plasma membrane and mainly localises to the lysosome after being endocytosed. Citing the prediction made by NetPhos2.0 which is based at the Technical University of Denmark, there are two probable phosphorylation sites within the active subdomain, a tyrosine residue at amino acid 268 (phosphorylation prediction 0.639) and a serine residue at aa 275 (phosphorylation prediction 0.931). Attempts to detect the phosphorylated amino acids in experiments with the chimera by immunoprecipitation followed by SDS-Page and Western blotting gave inconclusive results. A more promising approach for future investigation would be to mutate the serine/tyrosine residue to inphosphorylable amino acids such as alanine or phenylalanine or to a phosphomimetic such as glutamic acid and test these mutated constructs for their autophagic potential.

\section{AIR-induced autophagy depends on the class III PI3K, Vps34}

Given the different types of autophagy that have been described, it was important to further determine whether AIR was implicated in conventional macroautophagy.

As mentioned in the introduction (section 11), there are no truly specific pharmacological inhibitors for macroautophagy. However, the phosphatidylinositol-3-kinase (PI3K) inhibitors Wortmannin or 3-Methyladenine are often used for autophagy inhibition (Mizushima et al. 2010). In the canonical autophagic pathway there are two important PI3K involved, a class I PI3K that lies upstream of m-TOR and inhibits autophagy induction, and a class III PI3K (Vps34) that is found downstream of m-TOR and induces 
autophagsome formation. Therefore, the two PI3Ks implied in the pathway have contradictory effects which should be kept in mind when using pharmacological inhibitors of these molecules. The overall effect is usually an inhibition of autophagy, indicating that the effect on the autophagosome initiating complex (Beclin1/class3 PI3K) is stronger than on mTOR signalling, and these drugs are consequently often used to suppress macroautophagy (Mizushima et al. 2010). Additionally, if AIR was not involved in starvation induced autophagy, as hypothesized earlier, inhibition of the class I PI3K upstream of the nutrient sensor m-TOR could be irrelevant in the AIR signalling pathway and the use of Wortmannin and 3-Methyladenine would exclusively block the AIR-induced class 3 PI3K at the level of autophagosome formation.

In our experiment carried out with CD16:7-AIR chimeras (see figure 23), the unaggregated samples show no increase of lipidated LC3II when either Wortmannin or 3Methyladenine are added, suggesting that there is no autophagy inducing effect caused by these drugs. However, in the aggregated samples there is less detectable LC3II upon PI3K inhibitor treatment. This indicates that PI3K-dependent autophagosome formation is implicated in the process induced by AIR and thereby implies that AIR induces conventional macroautophagy at least in this context.

\section{AIR induced autophagy depends on Atg5 and Atg7 but probably not on Beclin1}

Apart from using pharmacological inhibitors, we also used a genetic approach to investigate whether the phenomenon induced by AIR was conventional macroautophagy. In this series of experiments, we used specific siRNAs to knockdown Atg5, Atg7 and Beclin1 and test the impact of this depletion in AIR signalling. Atg5 and Atg7 are involved in autophagosome elongation while Beclin1 forms part of a complex that initiates autophagosome formation (for further details see Introduction section 5.3.). Using LC3 as an autophagic marker in Western blot, we obtained the result that AIR-induced autophagy (examined in the context of the CD16:7 chimera) depended on Atg5 and Atg7 but not on Beclin1. Conventional macroautophagy is usually assumed to depend on all three of the proteins that we knocked down in these siRNA experiments.

\subsection{AIR does not induce Atg5/Atg7-independent autophagy}

Lately there have been studies describing either Atg5/Atg7-independent or Beclin1independent autophagy. However, our data indicates that AIR is unlikely to be involved in 
the type of Atg5/Atg7-independent macroautophagy lacking LC3-lipidation as was described by Nishida et al. (Nishida et al. 2009b) because AIR clearly induces formation of LC3II and depends on Atg5 and Atg7. Nevertheless, Beclin1 seems unnecessary for AIR-induced autophagy.

\subsection{AIR could be implicated in Beclin1-independent autophagy}

Similar to AIR-induced autophagy, Beclin1-independent autophagy (Scarlatti et al. 2008; Tian et al.) involves LC3 lipidation. In view of the fact that high ectopic expression of AIR induces non-apoptotic cell death, it is interesting to note that Beclin1-independent autophagy has been shown to play a role in a caspase-independent cell death in breast cancer cells (Scarlatti et al. 2008). Just as for AIR-induced autophagy, Atg7 is necessary for Beclin1-independent autophagy, but in contrast to our findings regarding AIR-triggered autophagy, Beclin1-independent autophagy is also Vps34-autonomous. AIR has been shown to depend on this PI3K as revealed by the CD16:7 chimera experiments in the presence of $\mathrm{PI} 3 \mathrm{~K}$ inhibitors. As mentioned before, PI3K-inhibitors are difficult to use for autophagy inhibition owing to the fact that there are two classes of PI3Ks with opposing effects on autophagy. Nevertheless, the results from our PI3K experiments seem credible and we currently cannot offer an explanation for these apparently contradicting data.

\section{Beclin1-independence of AIR-induced autophagy is unclear}

Previous reports have described that, in some cases, Beclin1 silencing prevents autophagosome formation, but not LC3II generation. For example, increased amounts of LC3II could be detected by Western blot in lysates of neonatal rat cardiac myocytes transfected with small hairpin RNA targeting Beclin1 and treated with rapamycin. Conversely, no GFP-LC3 punctae formation was induced. A similar phenomenon was observed in mouse embryonic stem (ES) cells: On comparing LC3II levels in the lysate of wild type (WT) cells with homozygously Beclin1-deficient cells by Western blotting, there was no decrease of lipidated LC3 in the samples from Beclin1-deficient cells, whereas transfected GFP-LC3 showed a punctuate distribution in WT cells but no GFP-marked punctae in Beclin $1^{-1-}$ cells. This indicates that although LC3 was conjugated to PE, there is no autophagy induction since there are no detectable autophagosomes. In contrast, in Atg5-deficient ES cells, LC3II formation measured by immunoblotting was completely blocked (Matsui et al. 2007). Consistent with these findings, in an Apg6-deficient yeast 
mutant (homologue for mammalian Beclin1) normal amounts of the lipidated (yeast homologue for) LC3 were detected in nutrient rich and starvation conditions. Punctuate structures that would likely represent autophagosomes, were however not detected in either condition (Suzuki et al. 2001). These findings imply that in Beclin1-deficient cells, LC3 is lipidated and therefore detectable as LC3II in Western blots. However, it seems that there is no formation of autophagosomes and therefore that neither binding of the lipidated LC3 to the autophagosome membranes nor its degradation occurs.

Considering these reports on LC3II generation without autophagosome genesis, we tried to further evaluate the notion that AIR induces autophagy independently of Beclin1, and therefore tested the capacity of AIR to stimulate the reporter GFP-LC3 in the absence of Beclin1.

\subsection{GFP-LC3 in fluorescence microscopy with the chimera}

To test if the LC3II formed in the presence of the active CD16:7 chimeras after silencing Beclin1 was participating in the generation of autophagosomes, we knocked down Beclin1 and transfected the chimeras together with GFP-LC3 for autophagosome quantification using fluorescence microscopy. Unfortunately, these experiments could not be evaluated because of an extremely high background in the negative controls. A reason for this could be unspecific aggregation of the transfected GFP-LC3. Although this phenomenon has been described before (Kuma et al. 2007), we did not expect this to happen in the chimera studies since our negative controls in the conventional overexpression experiments with full-length AIR and its deletions hardly showed any GFP-LC3-punctae. However, changing from ordinary AIR and LC3 co-transfections to the artificial receptorligand system with a highly glycosilated chimera CD16:7 required extensive refinement, and although the chimera functions well for studies in Western blotting experiments, it might reach its limitations when being used for microscopic studies. Therefore, it remains currently unclear whether the autophagic process induced by AIR is truly Beclin1independent.

\subsection{AIR and measuring the autophagic flux}

An indirect approach to test the validity of the result indicating that AIR-induced autophagy is Beclin1-independent could be to measure the autophagic flux. As mentioned in the introduction (see section 12.2.), elevated LC3II can mean both, increased production or decreased degradation of LC3II. In the case of LC3II forming without localising to autophagosomes, as described for Beclin1 knockdown (Matsui et al. 2007), protease 
inhibitors such as pepstatin or E64d or, alternatively, an inhibitor of lysosome acidification, BafilomycinA1 (see Introduction 12.2) would specify whether there is real LC3 turnover with functioning autophagy or not. In this scenario without autophagic flux, the nonautophagosomal LC3II would not be degraded, (contrarily to truly degradative autophagy) and these drugs would not be able to upregulate LC3II levels.

Our preliminary experiments with the chimeras and this type of drugs gave contradictory results, suggesting that the experimental setup was not completely refined. Apart from the fact that cells are usually incubated with these drugs for shorter times than the chimeras are aggregated for, pharmacological inhibition of autophagy has mostly been studied in cells undergoing starvation-induced macroautophagy and not, as in our case, cells undergoing autophagy that was activated by overexpression of signalling pathway inducers.

Thus, despite our attempts to further investigate the role of Beclin1 in AIR-induced autophagy, it remains problematic to predict whether Beclin1 is implicated in AIR signalling.

\subsubsection{Inhibitors of lysosomal acidification affect AIR beyond inhibition of autophagic degradation}

There are reports that Bafilomycin has other functions at lower concentrations at which it does not succeed in inhibiting autophagic degradation. In one report, Bafilomycin at low concentrations protected neurons against apoptotic cell death that had been induced as a consequence of pharmacological autophagy inhibition (Shacka et al. 2006). This shows that Bafilomycin has different effects depending on the concentration used and could also mean that Bafilomycin acts differently in the autophagic pathway according to the autophagy inducing stimuli. Additionally, as mentioned before when considering the protease inhibitors, the effect of this lysosomotropic agent on autophagic degradation has above all been studied in starvation induced autophagy (Rubinsztein et al. 2009).

It should also be kept in mind that another lysosomotropic agent, chloroquine, inhibited internalization of AIR and, therefore, most likely the downstream action of AIR inside the cell. Bafilomycin could also cause some effect on AIR trafficking that might, in turn, alter its ability to induce autophagy. In fact, although the effect was not as strong as with chloroquine, AIR could be detected on the cell surface of unpermeabilised SAOS- 2 cells after treatment with Bafilomycin A1 (data not shown), suggesting that Bafilomycin A1 to some extent also inhibited AIR endocytosis. Assuming that endocytosis is necessary for AIR-induced autophagy, treating cells with Bafilomycin $A 1$ to study the AIR-induced autophagic flux would be difficult as Bafilomycin A1 could inhibit both: AIR triggered 
autophagy and degradation in the autophagolysosome. When studying autophagy by means of LC3II amounts detected in Western blots, these two effects can probably cancel each other out, a notion that would explain our varying results when using this lysosomotropic drug to explore the autophagic flux in the context of the CD16:7-AIRchimera experiments.

\subsection{The autophagic vacuoles induced by AIR could be LC3-positive} phagosomes

AIR could be implicated in recently discovered autophagic processes important for immunity, phagocytosis or neuroprotection, as these generally also depend on the proteins involved in conventional autophagy such as Atg5/Atg7 and LC3. For example, the study by Sanjuan et al (Sanjuan et al. 2007) links autophagy and phagocytosis by showing that TLR-induced localisation of LC3 to the phagosomes depends on Atg5 and Atg7. These LC3-positive phagosomes were single-membraned. Considering this phenomenon as a feasible possibility of what is induced by AIR (as AIR induces LC3 lipidation and was also shown to depend on Atg5 and Atg7), our difficulties in using protease inhibitors to block LC3II degradation would be understandable. Firstly, the protease inhibitors E64d and Pepstatin could be less effective in preventing degradation in these LC3-positive phagosomes due to a different assembly of proteases in phagosomes when compared to autophagolysosomes (Fukazawa \& Koyama 1982). Secondly, in autophagolysosomes the inner membrane of the double-membraned autophagosome is degraded. Both autophagosome membranes are LC3 positive, therefore by degrading the inner membrane, LC3 is also degraded. In the study by Sanjuan et al. it was not specified on which side of the single-membraned phagosomes, LC3 was located. It is possible that in these phagosomes, LC3 degradation does not take place as there is no contact of the LC3 on the outer side of the single phagosome membrane with the content of the phagosome. Hence, in this scenario without LC3 degradation, inhibitors of LC3 degradation would be unlikely to show a strong effect.

\section{AIR has been reported to modulate protein glycosylation in the Golgi-apparatus}

The first characterisation of AIR that was published by Ullrich et al, while we were also working on this protein, concludes that AIR is important for the complex glycosylation of the amyloid precursor protein (APP) and its subsequent cleavage by $\alpha$ - and $\beta$-secretases 
(Ullrich et al. 2010). Overexpression as well as a knockdown of AIR caused inhibition of APP shedding. This at first sight contradictory finding was explained by the authors through pointing out that abundance as well as a lack of AIR could cause disturbance of this pathway. The mechanistic explanation provided by the authors for the effect of AIR manipulation on APP shedding is that, due to inhibiting the glycosylation of APP, AIR indirectly reduces the access of APP to cell compartments where it is usually cleaved by $\alpha$ - and $\beta$-secretases.

\subsection{APP-shedding and its impact on Alzheimer's disease}

As mentioned in the introduction (see section 7.1.), APP shedding plays a role in the neurodegenerative Alzheimer's disease (AD). On the one hand, shedding by the $\beta$ secretases leads to formation of the neurotoxic Abeta $(A \beta)$ peptide, the accumulation of which seems to contribute to neuronal cell death leading to AD (Yankner et al. 1990). On the other hand, shedding by the $a$-secretase leads to a neuroprotective fragment, the alpha-secretase cleaved APP (sAPPalpha) (Stein et al. 2004). However, Ullrich et al. showed that overexpression or knockdown of AIR led to inhibition of APP shedding by both secretases, the $\alpha$ - and $\beta$-secretases, which have contrary effects on neuroprotection in $A D$. Therefore the overall effect of AIR on the pathology of this disease currently cannot be predicted.

\subsection{AIR as an autophagy inducer and $A / R$ in Golgi-located glycosylation of APP seem incoherent}

Although Ullrich et al. did not mention autophagy in their study, it is highly interesting to note that there is emerging evidence of autophagy being implicated in Alzheimer's disease. Although the main reason for the protective effect of macroautophagy on $A D$ seems to be that $A \beta$ becomes degraded (Boland et al. 2010), there are, however, reports indicating that defective autophagy leads to vesicles in which not only degradation is impaired but also $A \beta$ generation takes place (Yu et al. 2005). Unfortunately, none of these findings can be correlated with a possible indirect influence of AIR on APP shedding through glycosylation events. Based on our own research, we hypothesised that AIR was implicated in non-starvation induced autophagy but we did not investigate the function nor did we find references for AIR acting in Golgi-based glycosylation. In fact, we found AIR to be expressed in endosomal-lysosomal compartments rather than in the Golgi-apparatus, and therefore we tend to believe that its main action takes place in its natural localisation. This argues against an activity in the Golgi-apparatus as proposed by Ullrich et al. Also, by 
narrowing down the active subdomain of AIR responsible for autophagy induction we identified a part showing little similarity to the corresponding amino acid sequence within the AIR homologue BSMAP. However, Ullrich et al. described similar findings for both homologues, thus indicating that the glycosylation function discovered by them is not related to the autophagy-inducing properties of AIR. 



\section{$V$ Conclusions}

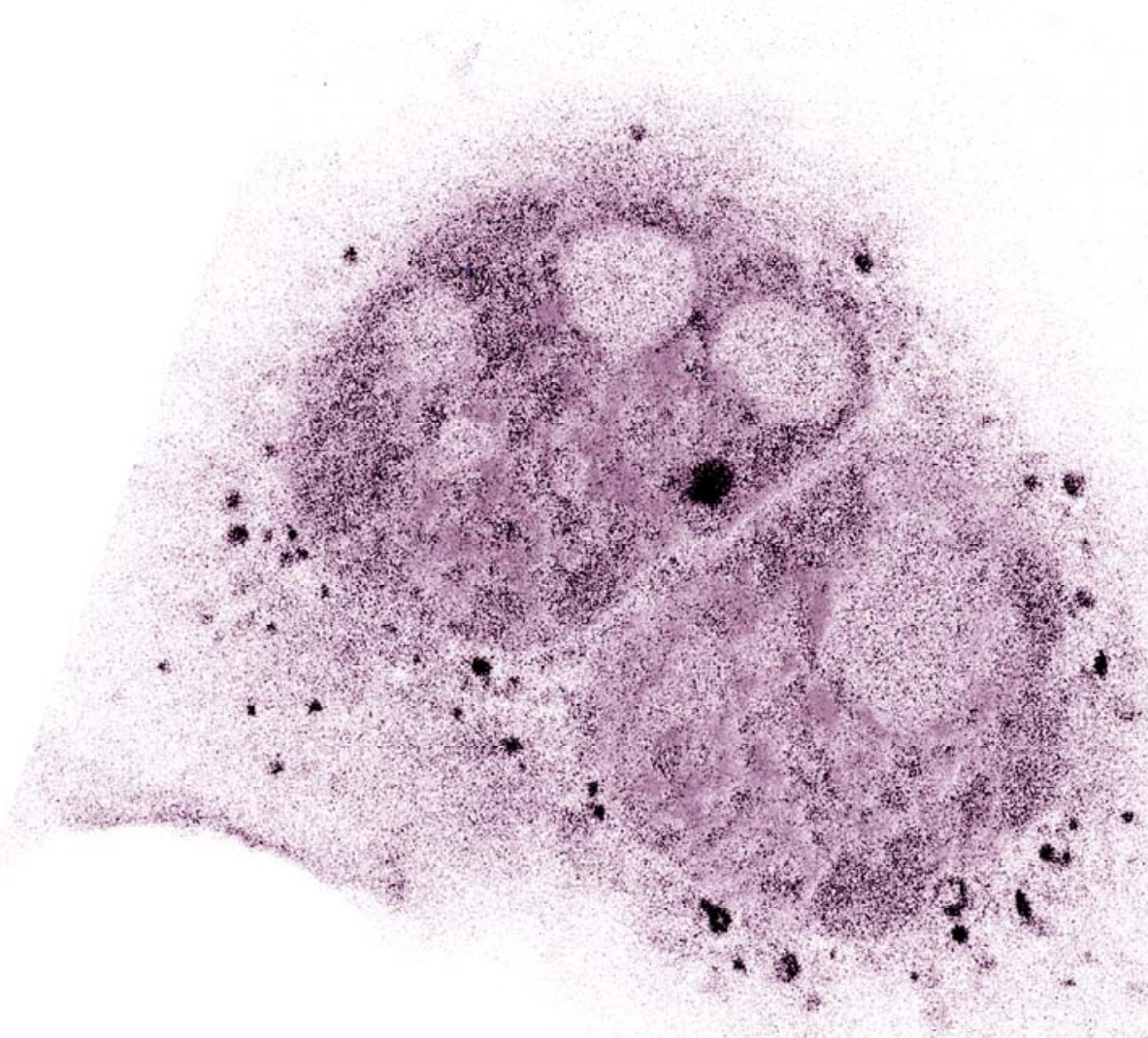



1. Overexpression of AIR in human cells induces autophagy.

2. Massive overexpression of AIR induces a caspase-independent type of cell death.

3. AIR is expressed at the cell membrane but is constantly and rapidly endocytosed.

4. The main subcellular location of AIR is the endosomal-lysosomal compartments.

5. The N-terminal of AIR is extracellular.

6. The C-terminal intracellular domain of AIR is necessary and sufficient for autophagy induction.

7. The active subdomain of AIR is within its amino acids $263-281$.

8. AIR-induced autophagy depends on the driving force behind autophagosome nucleation, the class III PI3-kinase Vps34.

9. AIR-induced autophagy depends on the central autophagy-related proteins Atg5 and Atg7. 



\section{Materials and Methods}

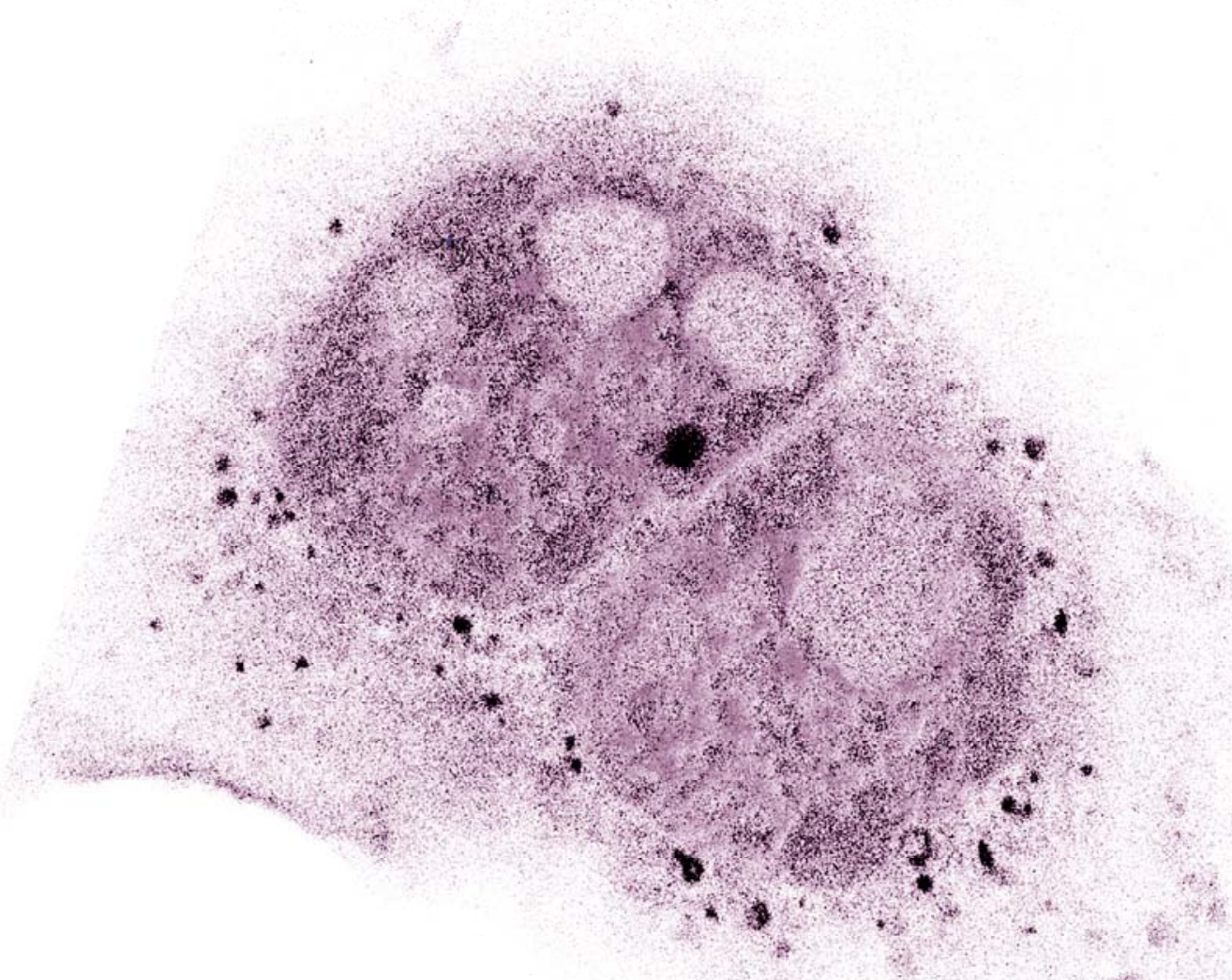



In the following, the conditions under which the experiments were carried out will be described. For further information on the materials used, please refer to the tables at the end of this section.

\section{Plasmids and plasmid construction}

\subsection{DNA amplification through polymerase chain reaction}

The cloning of a DNA sequence into a plasmid was carried out using the polymerase chain reaction technique (PCR). Templates were either already existing plasmids or a cDNA library. The primers contained endonuclease restriction sites which were thus inserted into the DNA sequence for subsequent digestion and ligation.

Prior to the PCR, the templates were digested by a restriction enzyme in order to linearise the DNA. This enzyme did not have restriction sites within the to-be-expected PCR band and was Spe I in most cases.

To increase success rates the PCRs were carried out with and without $10 \%$ DMSO addition in 1x Pfu buffer with $0.5 \mu \mathrm{g}$ DNA template, $0.25 \mu \mathrm{g}$ forward and reverse primer each, $10 \mathrm{nmol}$ deoxynucleotide triphosphates (dNTP) and $1.5 \mathrm{U}$ Pfu polymerase in a total volume of $50 \mu \mathrm{l}$.

The following default conditions were used for PCR cycles. The number $(X)$ of cycles depended on the template origin and was 12-14 for a plasmid and 15-35 for a cDNA from a library.

- One denaturation cycle for 4 minutes at $96^{\circ} \mathrm{C}$

- X amplification cycles
o 1 minute at $95^{\circ} \mathrm{C}$
o 1 minute at $56^{\circ} \mathrm{C}$
o 2-5 minutes at $72^{\circ} \mathrm{C}$

- Samples were kept at $4{ }^{\circ} \mathrm{C}$ until removal from the thermal cycler

Successful amplification of the desired band was confirmed by running part of the sample in a $1 \%$ agarose gel. The amplified fragment was visualized in a transilluminator with ultra violet light and compared to a $1 \mathrm{kbp}$ DNA marker. 
The remaining sample was purified using the QIAquick (Qiagen) purification kit according to the protocol provided by the manufacturer.

\subsection{Ligations}

The inserts were cloned into the pEAK8 (P8) or pEAK12 (P12) vector (Edge Bio). Both vectors carry the EF 1a promoter and resistance genes for ampicillin (prokaryotes) and puromycin (eukaryotes). The vector had restriction sites for various endonucleases, amongst them the restriction enzymes HIND III and Not I. These were the most frequently used sites for insertion of the DNA fragments to which the restriction sites had been added by PCR. After overnight digestion of the vector and the insert(s) with the corresponding enzymes at $37^{\circ} \mathrm{C}$ (0.5-1.5 $\mu \mathrm{g}$ DNA, $2 \mathrm{U}$ each per enzyme and appropriate buffer in $10 \mu \mathrm{l}$ total volume TE), the DNA fragments were separated in a low melting agarose gel $(1.2 \%$ NuSieve). To avoid intramolecular ligation reactions of the vector, it was dephosphorylated at the $5^{\prime}$ end by incubating with calf intestinal alkaline phosphatase (CIP) for 30 minutes at $37^{\circ} \mathrm{C}$ prior to gel loading ( $6 \mathrm{U}$ in $10 \mu$ total volume). The DNA fragments were visualized and cut out in a UV-transilluminator. For pipetting purposes the cut out agarose was heated up to $65^{\circ} \mathrm{C}$ and $1 \mu \mathrm{l}$ vector as well as $2 \mu \mathrm{l}$ insert $(1.5 \mu \mathrm{l}$ of each insert in the case of a three piece ligation) were added to the $37^{\circ} \mathrm{C}$ warm ligation mix containing:

\section{$0.5 \mu \mathrm{BSA}$}

$1.25 \mu \mathrm{l} 10 \mathrm{x}$ ligation buffer

$0.3 \mu \mathrm{l}(120 U)$ T4 DNA ligase

\section{$8.2 \mu \mathrm{l}$ MilliQ water}

The ligation mix was incubated over night at room temperature. The vector on its own (or with only one insert in the case of a three piece ligation) served as a negative control.

For transformation the competent bacteria from the Escherichia coli strain $\mathrm{DH} 5$ a were used. These bacteria had been prepared by the sterilization department at the Centre of Cancer Research, Salamanca, following a previously published protocol (Inoue et al. 1990) and transformation of the ligated plasmid was done using the heat shock method. Accordingly, the competent cells were incubated with the ligation for 30 minutes in ice and subjected to a heat shock in a water bath at $42^{\circ} \mathrm{C}$ for 45 seconds after which, the bacteria were returned into the ice. After a minimum of two minutes the cells were plated out onto LB-Agar plates ( $2 \%$ agarose in Luria Bertani medium that contains $0.5 \%$ yeast extract, $1 \%$ sodium chloride and $1 \%$ tryptone peptides) with ampicillin $(50 \mu \mathrm{g} / \mathrm{ml})$ which ten 
minutes before had been covered with a thin coat of antibiotic-free $1.5 \%$ LB-Agar. The amount of bacteria and ligation depended on the complexity of the ligation and ranged from 50 to $100 \mu$ l bacteria and from 2 to $5 \mu$ ligation. Colonies were grown over night at $37^{\circ} \mathrm{C}$ and various of them were picked the next day. For further growth, the picked colonies were grown for $8-14 \mathrm{~h}$ in liquid, ampicillin containing $(50 \mu \mathrm{g} / \mathrm{ml})$ LB medium. The bacterial pellet from $3 \mathrm{ml}$ medium was processed with the QIAprep ${ }^{\circledR}$ Minispin kit according to the protocol provided in order to purify the plasmid DNA. Before inoculating for larger scale bacteria growth the miniprep DNA was digested and analysed by electrophoresis. On showing positive for the desired ligation product, midi- (100ml LB) or maxipreps (400ml LB) were inoculated depending on the predicted use of the plasmid. These bacterial cultures were allowed to grow for $16-20 \mathrm{~h}$ at $37^{\circ} \mathrm{C}$ under rigorous shaking (250rpm).

Plasmid purification was carried out using a Qiagen midi or maxi kit. Precipitated DNA was dissolved in TE buffer to give a plasmid concentration from 1-2 $\mu \mathrm{g} / \mu \mathrm{l}$. Exact concentration of the DNA preparation was measured with an UV-Vis-spectrometer at $260 \mathrm{~nm}$. Additionally, the DNA was analysed by electrophoresis without and after digestion with endonucleases as well as by sequencing, which was carried out by the genomics department at the Centre of Cancer Research, Salamanca, (3100 Genetic Analyzer). To analyse the sequences, programs such as Edit Seq and MapDraw (DNAstar package) were used.

\subsection{Plasmids}

Most plasmids used for this thesis contained either the pEAK8 (P8) or the pEAK12 (P12) vector. pEAK vectors express the inserts below the control of the promoter gene EF1a and were distributed by Edge Bio Systems.

For retroviral transductions the vector P12-MMP was used, which is a pMMP (Dr. Richard Mulligan) derivative that Dr. Felix Randow kindly shared with us. Further, pMD-gag-pol and pMD-VSV-G were used for virus production in HEK-293T cells, both of them were a gift from Dr. Richard Mulligan.

For growth of a GST-fusion-protein in bacteria, its encoding DNA sequence was introduced into the pGEX-4T vector (GE Healthcare). 


\subsection{1. $P 8$ and $P 12$ vectors}

0 P12-Poly

This plasmid was used as a negative control and filler. It is a pEAK12 vector with a poly linker site as an insert.

\section{P8-GFP-LC3A/B}

Human LC3A and LC3B were cloned from a human T cell blast CDNA library. Through the PCR, Pci I and Not I restriction sites were inserted into the cDNA and subsequently used to ligate the DNA sequence with a pEAK8 vector carrying GFP (green fluorescent protein).

\section{o P8-HA-LC3 A/B}

The HA tag was obtained by annealing of the oligonucleotides and the tag was subsequently attached to LC3. The TOP and BOTTOM oligonucleotides needed to be phosphorylated in order to be able to ligate the cDNA. This was done at $37^{\circ} \mathrm{C}(1.5 \mathrm{~h})$ after addition of PNK buffer, ATP $(1.25 \mathrm{mM})$ and T4 kinase $(1.25 \mu \mathrm{l})$ in a total volume of $10 \mu \mathrm{l}$. The kinase was inactivated through a 30 minute incubation at $70^{\circ} \mathrm{C}$ after which the oligonucleotides were mixed and heated up to $100^{\circ} \mathrm{C}$ for two minutes. Then $2.5 \mu \mathrm{l} 10 \mathrm{x}$ annealing buffer was added and the mixture was allowed to cool down slowly to room temperature. P8-GFP-LC3 (see above) was digested with Hind III and Pci I to liberate GFP and to use the vector with LC3 for the ligation of the HA tag into the N-terminal of LC3.

\section{o P8-BNIP3L}

The BNIP3L plasmid comes from the cDNA library used in the screening by Alcalá et al. 2008 and contains the entire cDNA encoding for the protein BNIP3L.

\section{o P12-AIR}

AIR was cloned from the cDNA library clone 9-1.4-P15 identified in the screening by Alcala et al, 2008. Using this original clone as a template, PCR was used to amplify and clone the corresponing open reading frame into pEAK12. The oligonucleotides used for the PCR were designed to introduce the restriction sites for Hind III and Not I which were subsequently used to ligate the insert with the vector pEAK12. 
o P12-CD5-HA-AIR

In order to remove the putative leader sequence (until E36) of the cDNA clone 9-1.4-P15 and introduce the restriction sites Pci I and Not I, a PCR with a forward primer Pci I-AIRE36-fw and a reverse primer Not I-Stop-AIR-rev was carried out with Spe I digested 91.4-P15. Using BspLU and Not I for insertion, the newly created fragment was ligated into a P12 vector carrying CD5-HA, which had been obtained by digestion of P12-erRFP.

o P12-AIR $\Delta$ 263; P12-AIR $\Delta$ 268; P12-AIR 2 274; P12-AIR $\Delta 282 ;$ P12-AIR $\Delta 290 ;$ P12-AIRA299; P12-AIRA307; P12-AIRA317; P12-AIRA319

All AIR deletions were cloned by PCR with P12-AIR as a template and inserted into pEAK12 using Hind III and Not I as restriction sites.

o P12-GST

This plasmid was used as a transfection control due to the expression of the gluthathioneS-transferase from Schistosoma japonicum.

O P12-CD16:7; P12-CD16:7-AIR-ID (=P12-CD16:7-AIR-263-323); P12-CD16:7AIR-IDAR290 (=P12-CD16:7-AIR-263-289); P12-CD16:7-AIR-IDAF282 (=P12CD16:7-AIR-263-281); P12-CD16:7-AIR-Y268-323; P12-CD16:7-AIRY268DR290 (P12-CD16:7-AIR-268-289); P12-CD16:7-AIR-Y268DF282 (P12CD16:7-AIR-268-281); P12-CD16:7-AIR-F282-323; P12-CD16:7-AIR-R290323

The CD16:7 chimera had previously been constructed in the laboratory of Dr. Brian Seed (Romeo et al, 1992). The 16:7 cassette contains the extracellular part of the surface molecule CD16 (FcRyllI) fused to the transmembrane region of CD7, and is expressed at the plasma membrane because of the leader region of CD16. The original CD16:7 plasmid came with a sequence that included Hind III and Mlu I restriction sites which were used for digesting and liberating the CD16:7 cassette from its vector. The intracellular part of AIR and its deletions were cloned by PCR from P12-AIR with primers that inserted a Mlu I restriction site at the 5'end of the AIR sequence and maintained or inserted a STOP codon followed by a Not I restriction site at the 3'end. CD16:7 and the AIR sequence were ligated into a pEAK12 vector in a three piece ligation using the Hind III, Mlu I and Not I restriction sites. In the case of P12-CD16:7, the chimera was ligated with the vector and a Mlu I/Not I adaptor that included a STOP codon. 


\subsubsection{Vectors for retroviral transduction}

O P12-MMP-AIR-HA-IRES-Puro

This plasmid originates from a P12-AIR-HA plasmid which had been constructed by ligating AIR into a P12 vector with a C-terminal HA-tag. To add the Pci I restriction site that is needed for this ligation to the C-terminal of AIR, a PCR with the primers Pci I-revAIR and AIR-fw was carried out. Taking advantage of a Bgl II restriction site within the sequence of AIR, the PCR product was digested with $\mathrm{Bgl} I \mathrm{PCi} I$ and the resulting DNA fragment which encodes for the C-terminal part of our protein, was sequenced (see above). In a three piece ligation this DNA fragment was linked with the cut off AIR part, an insert from P12-AIR digested with Hind III/Bgl II, and ligated into P12-ct-HA using its Hind III and Pci I sites. Once the P12-AIR-HA plasmid had been cloned, AIR-HA was inserted into a P12-mmp-IRES-Puro vector using the Hind III, Not I and BspH I sites.

\section{o P12-mmp-Poly}

This vector is the $\mathrm{P} 12-\mathrm{mmp}$ vector with a poly linker site as an insert.

O pGEX-4T and pGEX-4T-AIR-E36-G239

The GST-AIR fusion protein containing the predicted extracellular domain of AIR was used for antibody production.

The AIR deletion was cloned by PCR as a truncated protein lacking the first 35 amino acids (the predicted leader sequence), the transmembrane and the intracellular parts. This AIR deletion was inserted in frame into the pGEX-4T vector (GE Healthcare) using the restriction sites EcoR I and Not I.

\section{Antibodies against AIR}

As there is no commercial antibody available for AIR we needed to produce one ourselves. In order to do so the extracellular part of AIR lacking the leader sequence was grown in bacteria as a GST fusion protein using the plasmid pGEX-4T-AIR-E36-G239 (see 1.3.). The standard extraction procedure for GST-fusion proteins had to be modified according to (Frangioni \& Neel 1993) because the GST-AIR fusion protein was insoluble. This method uses the alkyl anionic detergent $\mathrm{N}$-laurylsarcosine as a sodium salt (sarkosyl) which can solubilise the GST-AIR mutant.

The protein was produced in competent bacteria from the Escherichia coli strain BL21 which were cultured in LB with ampicillin $(50 \mu \mathrm{g} / \mathrm{ml})$. The bacterial pellet was 
resuspended in STE buffer $(50 \mathrm{mM} \mathrm{NaCl}=$ sodium chloride; $10 \mathrm{mM}$ Tris $\mathrm{pH}$ 8.0; $1 \mathrm{mM}$ EDTA) containing aprotinin $(2 \mu \mathrm{g} / \mathrm{ml})$, leupeptin $(2 \mu \mathrm{g} / \mathrm{ml})$ and PMSF $(1 \mathrm{mM})$ and lysed by adding lysozyme in Tris-buffer pH 8.0 to reach a final concentration (f. c.) of $100 \mu \mathrm{g} / \mathrm{ml}$, as well as DTT (f. c. $5 \mathrm{mM}$ ) and sarkosyl (f. c. $1.5 \%$ ). After centrifugation, TX100 was added to reach a f. c. of $2 \%$ and the lysate was incubated with $\mathrm{GSH}$-beads for $3 \mathrm{~h}$ at $4{ }^{\circ} \mathrm{C}$ which were then washed with NET-T buffer (NET: $150 \mathrm{mM} \mathrm{NaCl} ; 1 \mathrm{mM}$ EDTA; $20 \mathrm{mM}$ Tris $\mathrm{pH} 8.0$ with additional $1 \%$ TX100) before being applied to a Bio-Rad column. After washing with NET, the protein was eluted using $50 \mathrm{mM}$ reduced glutathione and $120 \mathrm{mM}$ $\mathrm{NaCl}$ in $100 \mathrm{mM}$ Tris $\mathrm{pH}$ 8.0. Part of the protein was sent to Eurogentec, Belgium for rabbit and chicken immunisation. The other part $(\approx 3 \mathrm{mg})$ was used for affinity purification using the AminoLink Plus Immobilization Kit from Pierce. To remove primary amines, the eluated protein was submitted to dialysis in a Slide-A-Lyzer dialysis cassette (MWCO $7 \mathrm{kDa}, 0.5-3 \mathrm{ml}$ capacity, Pierce). After dialysis GST-AIR was coupled to the column provided by the AminoLink Plus Immoblilzation Kit following the steps for the protocol at $\mathrm{pH} 7.2$.

As the chicken antibody is obtained from eggs, the egg yolk was prepared and delipidated with the Eggcellent Chicken IgY kit (Pierce catalogue number 44918) prior to further purification. To eliminate GST recognising antibodies from the chicken and rabbit sera, they were passed through a column with immobilized GST (Pierce catalogue number 20205). After passing through the first column, the serum was applied onto the GST-AIR coupled column. The column was washed with PBS (phosphate buffered saline) and high salt PBS $(0.5 \mathrm{M} \mathrm{NaCl})$ before eluting in $0.1 \mathrm{M}$ glycine $* \mathrm{HCl} \mathrm{pH} 2.5$ and quick neutralisation in $1 \mathrm{M}$ Tris $* \mathrm{HCl} \mathrm{pH}$ 8.8. Different fractions were collected and spotted onto a nitrocellulose membrane for staining with Ponceau's reagent (Sigma: 0.1\% Ponceau S \{3-Hydroxy-4(2-sulfo-4-[4-sulfophenylazo]phenylazo)-2,7-naphthalenedisulfonic acid sodium salt $\}$ in $5 \%$ acetic acid). Protein containing fractions were combined and sodium azide was added (f. c. $0.1 \%$ ) for storage at $4^{\circ} \mathrm{C}$.

\section{Cell culture}

\subsection{Cell lines}

The HEK-293 (human embryonic kidney) cells, the 293 derivate HEK-293T, the SAOS-2 (human osteosarcoma) cells, HeLa (human cervical cancer) and the cell line JAR (human placenta choriocarcinoma) were supplied by ATCC (American Type Culture Collection). The cell line HCT 116 (human colorectal carcinoma) was a gift from Dr. Bert Vogelstein 
(Johns Hopkins University School of Medicine, Baltimore, Maryland, USA). All cell lines were cultured in DMEM (Dulbecco's modified Eagle's medium) supplemented with Lglutamine $(2 \mathrm{mM})$, penicillin $(100 \mathrm{U} / \mathrm{ml})$, streptomycin $(100 \mu \mathrm{g} / \mathrm{ml})$ and $10 \%$ foetal bovine serum previously inactivated during 30 minutes at $56^{\circ} \mathrm{C}$. The cells were kept at $37^{\circ} \mathrm{C}$ in a $5 \% \mathrm{CO}_{2}$-atmosphere.

\subsection{Stable cell line SAOS-2-AIR-HA}

This cell line was obtained through infection of SAOS-2 with a retrovirus leading to expression of AIR-HA and a resistance gene against Puromycin in order to be able to select the infected cells.

\subsubsection{HEK-293T-AU1BCIXL-CrmA-p35}

The retrovirus was produced in a cell line which expressed apoptosis inhibitors in order to cope with production of cell death inducing viruses and therefore led to a better viral yield. These cells were HEK-293T cells which stably expressed the apoptosis inhibitors Bcl- $X_{L}$ (Boise et al. 1993), CmrA (Ray et al. 1992; Callus \& Vaux 2007) and p35 (Clem \& Miller 1993). Generation of this cell line including infection, selection in puromycin (0.5 $\mu \mathrm{g} / \mathrm{ml})$, hygromycin $(0.2 \mu \mathrm{g} / \mathrm{ml})$ and neomycin $(0.5 \mathrm{mg} / \mathrm{ml})$ and cultivation of single colonies up to obtaining single clone cell populations was carried out by Martina Klee at our laboratory (Klee 2008). Selection of the single clone population was based on viability studies in which cell death inducers were co-transfected with GFP making it possible to detect cell death via fluorescence microscopy and confirmation of apoptosis inhibitor expression was achieved by Western blotting.

\subsubsection{Generation of SAOS-2 stably expressing AIR-HA}

In order to stably infect the SAOS-2 cells with AIR-HA, the HEK-293T-AU1BcIXL-CrmAp35 cells described above were transfected with the calcium phosphate method (see 6.1.). For a six-well-plate well, a total of $5.1 \mu \mathrm{g}$ DNA were used, $2.5 \mu \mathrm{g}$ of which were the to-be-transduced plasmid P12-mmp-AIR-HA-IRES-Puro, carrying an internal ribosome entry site (IRES) followed by a puromycin resistance gene, 0.7 $\mu \mathrm{g}$ pMD-VSV-G (vesicular stomatitis virus $G$ protein), leading to expression of the protein $G$ of the virus envelope, and $1.9 \mu \mathrm{g}$ pMD-MLV-OGP which codifies for the gag and pol proteins that are important for the structure of the virus and its reverse transcriptase. Another well was transfected with the same quantities of pMD-VSV-G and pMD-MLV-OGP as well as with $2.5 \mu \mathrm{g}$ P12mmp-Poly. The resulting virus was for infection of SAOS-2 which would be used as a 
control in a selection process posterior to infection. $24 \mathrm{~h}$ after transfection, the cell medium (supplemented DMEM) was changed and left as $2 \mathrm{ml}$ per well. At the same time, the target cells for infection, SAOS-2, were plated out in six well plates to be around $50 \%$ confluent at the moment of transduction, 24 hours later. For infection, the supernatants of the P12-mmp-Poly and P12-mmp-AIR-HA-IRES-Puro virus producing cells were passed through a $0.45 \mu \mathrm{m}$ filter (Whatman FP30/0.45 Ca-S) and added to the same amount of fresh cell medium. The DMEM on the SAOS- 2 cells was replaced with this mixture and subsequently the SAOS-2 were centrifuged in an Eppendorf centrifuge for cell culture plates at $700 \mathrm{~g}_{\mathrm{n}}$ and $32^{\circ} \mathrm{C}$ for 60 minutes before returning them to the cell incubator. The following day, the cells transduced with either P12-mmp-Poly or P12-mmp-AIR-HA-IRESPuro were put into puromycin $(0.6 \mu \mathrm{g} / \mathrm{ml})$ containing supplemented DMEM medium and maintained in the tissue culture. From the time point at which all P12-mmp-Poly infected cells had died, the cells grown from P12-mmp-AIR-HA-IRES-Puro infected SAOS-2 were believed to be stably expressing AIR-HA and used for experiments.

\section{Storage in liquid nitrogen}

In case of prolonged cell storage, cells were kept frozen in supplemented DMEM with $10 \%$ DMSO and stored in liquid nitrogen $\left(-196^{\circ} \mathrm{C} / 77 \mathrm{~K}\right)$.

\section{Cell splitting}

In general, cells were split every two to three days. After rinsing with PBS, cells were trypsinised for 3-5 minutes and supplemented DMEM was added to quench the trypsin and collect the cells by pipetting. Placed in a falcon tube, these cells were centrifuged at $600 \mathrm{~g}_{\mathrm{n}}$ for 5 minutes in order to remove the DMEM with the trypsin so the cells could be resupended in fresh medium and a fraction of them could be plated out in a new cell dish.

\section{Transfections}

Most transfections were carried out using the cationic lipid JetPEI although in some experiments HEK-293 cells were transfected with calcium phosphate.

In all protocols the cells were split a day before transfection to be around $50 \%$ confluent for JAR, HCT116 and HEK-293 cells and around 70\% confluent for SAOS-2 at the moment of transfection. For experiments in which AIR and its mutants were overexpressed together with GFP-LC3 for fluorescence microscopy, the cell lines SAOS-2, Jar and HCT 116 were seeded into 24-well plates containing poly-L-lysine coated coverslips. 
HEK-293 cells were directly seeded into 24-well plates as these cells were later centrifuged onto microscope slides in a cytospin centrifuge. For the Western blotting experiments with HA-LC3 transfected cells, 6-well plates were used.

In the case of transfection with the chimera, cells were split again a day after transfection, in order to have one well for aggregation with anti-CD16 antibodies and another well as a control.

\subsection{Calcium phosphate precipitation}

This method works through co-precipitation of the DNA together with calcium phosphate, the visible pellets of which are then taken up by the cells. The DNA for transfection was added to a $0.5 \mathrm{M} \mathrm{CaCl}_{2}$ solution (tissue culture grade, f. c. $0.25 \mathrm{M}$ ) in MilliQ sterile water. To this mixture, the same amount of 2 *HEBS $\left(280 \mathrm{mM} \mathrm{NaCl} ; 1.5 \mathrm{mM} \mathrm{Na}_{2} \mathrm{HPO}_{4} ; 11 \mathrm{mM}\right.$ glucose and 50mM HEPES, $\mathrm{pH}$ 7.1) was added. Depending on the desired size of precipitate, the solutions were mixed by pipetting 2-20 times and added dropwise to the cell containing wells. Amounts used for transfection of a six-well plate well were $5 \mu \mathrm{g}$ DNA in $250 \mu \mathrm{CaCl}_{2}$-HEBS mixture.

\subsection{Transfection with JetPEI}

This reagent is a cationic lipid that forms liposomes with the DNA. These liposomes can enter the cell by fusion with the cell membrane.

The DNA and JetPEl were diluted separately in an endotoxin free $150 \mathrm{mM} \mathrm{NaCl}$ solution according to the protocol provided by the manufacturer (PolyPlus). After combining the DNA solution with the JetPEI solution, the mixture was allowed to incubate for 40 minutes before adding it dropwise to the cells.

\subsection{Over-expression of AlR and its mutants}

In 6-well plate wells JAR and HEK-293 cells were co-transfected with 0.5 $\mu$ g P12-GST, $0.5 \mu \mathrm{g} \mathrm{HA}-\mathrm{LC} 3$ and $2 \mu \mathrm{g}$ AIR plasmid. P12-Poly plasmid was added to reach a total amount of DNA per well of $5 \mu \mathrm{g}$. BNIP3L was always co-transfected with a plasmid expressing the viral caspase inhibitor P12-p35 to prevent apoptotic cell death. Due to lower expression levels in SAOS-2, $1 \mu \mathrm{g}$ HA-LC3, 0.5 $\mu \mathrm{g}$ P12-GST and 3.5 $\mu \mathrm{g}$ AIR were used for transfection in these cells. In 24-well plates, cells were transfected with a fifth of the quantities for 6-well plates and, for fluorescence microscopy GFP-LC3 was used as an autophagic marker instead of HA-LC3. 


\subsection{Aggregation of the chimera with an anti-CD16 antibody}

HEK-293 cells were transfected in six-well plates with a total of $5 \mu \mathrm{g}$ DNA (1.5 $\mu \mathrm{g}$ CD16:7;

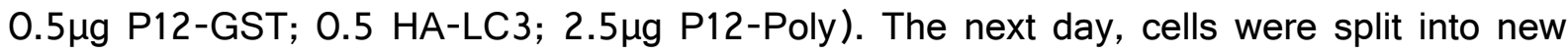
plates (two wells per experimental point: one to be aggregated and one as an unaggregated control) and 16-20 hours later (i.e. 40h post transfection) the anti-CD16 antibody was added in supplemented DMEM to give a final concentration of $4 \mu \mathrm{g} / \mathrm{ml}$. Previously, medium was removed from the wells and fresh medium only was added to the control well. After a 10 minute incubation at $37^{\circ} \mathrm{C}$ and $5 \% \mathrm{CO}_{2}$, a secondary polyclonal anti-mouse antibody was added with complete DMEM to reach a final concentration of $10 \mu \mathrm{g} / \mathrm{ml}$. Cells were lysed 24 hours later.

\subsection{P/3-kinase inhibitors}

To inhibit autophagy with the PI3K inhibitors 3-Methyladenine (3-MA) and Wortmannin (WM), these were added to the chimera-transfected HEK-293 30 minutes before aggregation with the antibody. 3-MA was used at a final concentration of $10 \mathrm{mM}$. It was directly dissolved in supplemented DMEM to achieve a $20 \mathrm{mM}$ stock solution. WM was used at a concentration of $0.2 \mu \mathrm{M}$. The stock dissolved in DMSO was $2 \mathrm{mM}$. WM was readded after 11 hours. 3-MA and WM solutions were sterile-filtered before use $(0.2 \mu \mathrm{m}$ filter FP $30 / 0.2$ cellulose acetate).

\subsection{Inhibition of endocytosis with chloroquine}

The AIR surface stainings of SAOS-2 were carried out after a $24 \mathrm{~h}$ incubation with chloroquine. Chloroquine diphosphate (Sigma) was dissolved in tissue culture grade water $(50 \mathrm{mM})$. This stock solution was added to the cells to give a final concentration of $50 \mu \mathrm{M}$.

\subsection{Transfections with siRNAs}

Atg5, Atg7, Beclin1 and AIR were knocked down by siRNA (small interfering RNAs). For every to-be-silenced RNA transcript, four double-strand RNA oligonucleotides were designed and supplied by Dharmacon (OnTarget Plus). As a control, a blend of four double stranded siRNAs without complete homology to any human gene was used. One day before transfection, cells were split into 6-well plates using complete DMEM lacking antibiotics.

In the chimera experiments (Atg5, Atg7, Beclin1) the siRNA was transfected in the morning using the lipid transfection reagent DharmaFECT1 following the instructions 
provided. The cells were subsequently transfected 10-11 hours later with the DNA encoding for the chimeras, LC3 and GST using the JetPEI transfection reagent. The next day, the cells were split into two wells, thereby obtaining one point for aggregation with the artificial ligand, an antibody against CD16 and one point as a negative control for the aggregation experiment.

\section{Cell lysis}

Cells were collected either by simple pipetting (HEK-293) or by trysinisation (HCT116, HeLa, JAR and SAOS). Trysinisation proceeded for 5 minutes $\left(37^{\circ} \mathrm{C} ; 5 \% \mathrm{CO}_{2}\right)$ and cells were detached by pipetting after adding supplemented cold DMEM. The collected cells were kept in ice and centrifuged at $4^{\circ} \mathrm{C}$ and $500 \mathrm{~g}$ for 5 minutes (Beckmann centrifuge). After washing with cold PBS, cells were lysed for 30 minutes in a lysis buffer containing a non-ionic detergent (50mM Tris, $\mathrm{pH} 7.5 ; 150 \mathrm{mM} \mathrm{NaCl} ; 5 \mathrm{mM}$ EDTA; $1 \%$ [v/v] Igepal CA-360). Before use, $10 \%$ of a protease inhibitor cocktail (Sigma) with 4-(2aminoethyl)benzenesulfonyl fluoride (AEBSF), pepstatinA, E-64, bestatin, leupeptin and aprotinin, as well as phenylmethyl sulphonyl fluoride (f. c. $10 \mu \mathrm{g} / \mathrm{ml}$ ) were added to the lysis buffer. Cell nuclei were separated from the lysate by centrifugation in a table top centrifuge (Eppendorf) at $4^{\circ} \mathrm{C}$ and $132000 \mathrm{rpm}$ for 10 minutes. The lysate was mixed with the same amount of $2 x$ concentrated loading buffer (100mM TRIS [pH 6.8]; 10\% ßmercaptoethanol; 4\% SDS; $0.2 \%$ bromphenol blue; $20 \%$ glycerol in MilliQ water) and boiled for 10 minutes in a heating block at $100^{\circ} \mathrm{C}$. It was found to be advisable to denaturalize the lysate before freezing it for storage because of the instability of the LC3. A small amount of lysate was kept aside to measure the protein concentration with the colorimetric BioRad Dc Protein Assay. This method is similar to the Lowry method (Lowry et al. 1951), which is based on the reaction of protein with an alkaline copper tartrate solution and Folin reagent. Colour development is primarily due to the amino acids tyrosine and tryptophan, and to a lesser extent, cysteine, and histidine. Proteins effect a reduction of the Folin reagent, thereby producing reduced species with a characteristic blue colour with maximum absorbance at $750 \mathrm{~nm}$ and minimum absorbance at $405 \mathrm{~nm}$. For every measurement a new calibration curve was made up, plates were read out in the Tecan Ultra Evolution at 690nm. 


\section{Immunoprecipitation}

In order to detect endogenous AIR in Western blots it had to be precipitated from the full lysate. The cells were lysed in 1\% Igepal CA-360 buffer and the protein levels were measured as usual. To $0.3-0.5 \mathrm{~g}$ protein in $0.3 \mathrm{ml}$ lysis buffer, $5 \mu \mathrm{l}$ purified rabbit antiAIR serum were added and the mixture was incubated on a rotating wheel overnight at $4{ }^{\circ} \mathrm{C}$. The next day, $30 \mu \mathrm{l}$ protein A sepharose suspension (GE Healthcare) were added to each sample for precipitation of the anti-AIR antibody bound AIR. As the resin is provided in PBS and azide, it had to be washed with lysis buffer prior to addition and incubation with the lysate. The immunoprecipitations were left on a rotating wheel for 1 hour at $4^{\circ} \mathrm{C}$ and were subsequently washed four times by sedimentation of the resin in a microcentrifuge for approx. 5 seconds followed by removal of the supernatant and replacement with lysis buffer. After the last wash, as much liquid as possible was taken off the resin, $25 \mu \mathrm{l} 2 \mathrm{x}$ RSB were added to each sample and the mixture was boiled for 10 minutes. If not loaded onto a SDS gel immediately, the samples were stored at $-70^{\circ} \mathrm{C}$ until used.

\section{Western blotting}

\subsection{SDS-Page}

The protein extracts were run in a SDS-Page gel to separate them according to their size. Depending on the size of the to-be-detected protein, gels contained $10-14 \%$ polyacrylamide (Protogel 30\%: acrylamide 0.8\%, bisacrylamide stock solution 37.5 : 1). The system used for running Western blots was the Mini-PROTEAN ${ }^{\circledR}$ II Electrophoresis Cell (BioRad), and with the help of the Amersham Rainbow coloured protein molecular weight marker, the approximate molecular weight of the proteins could be determined. The running buffer contained $25 \mathrm{mM}$ TRIS, $200 \mathrm{mM}$ glycine and $0.05 \%$ SDS. Gels were run at constant voltage (100V) at room temperature.

\subsection{Wet transfer}

After running the SDS-Page gel the proteins were transferred onto a PVDF (polyvinylidene difluoride) membrane by electric current (90V, 120 minutes) in a wet transfer using the Mini Trans-blot ${ }^{\circledR}$ electrophoretic transfer system (Bio Rad) and a transfer buffer containing $25 \mathrm{mM}$ TRIS, $192 \mathrm{mM}$ glycine and $20 \%$ methanol in MilliQ water. 
In some cases transfer efficiency was checked by Ponceau staining (see section 2.) which could be easily removed from the membrane with TBS-tween (TRIS-buffered saline with $0.05 \%$ tween-20).

\subsection{Antibody incubation}

Prior to incubating with antibodies, the membrane was blocked with $10 \%$ non-fat dried milk diluted in TBS-T for a minimum of $1 \mathrm{~h}$ at room temperature. Primary antibodies were diluted in $2 \%$ BSA (bovine serum albumin) and $0.1 \%$ sodium azide in TBS-tween solution. After blocking, blots were washed 4 times for 5 minutes with TBS-tween before submitting them to incubation with the primary antibody. The membrane was left with the primary antibody in a dish placed on a shaker for overnight incubation at $4^{\circ} \mathrm{C}$. Subsequently, the membrane was washed 4 times for 10 minutes and the freshly prepared secondary antibody solution $\left(5000^{-1}\right.$ - dilution in $5 \%$ milk TBS-tween) was added for a one hour incubation at room temperature. The secondary antibodies were conjugated to a horseradish peroxidase (HRP) which made subsequent chemiluminescent detection of the protein possible.

\subsection{Developing}

Depending on signal strength, the ECL (strong signals) or the ECL plus (weak signals) blotting detection systems (GE Healthcare) were used together with SuperRX films (Fuji Medical X-Ray Film, Fujifilm, Japan) for developing.

After development, the membranes were frozen at $-20^{\circ} \mathrm{C}$ for possible future reprobing or incubated directly with another antibody. To remove signals from the previous antibody incubation, the membranes were stripped with $7 \mathrm{M}$ guanidine in MilliQ water and blocked again in $10 \%$ milk TBS-tween.

\section{Fluorescence microscopy}

\subsection{Fixation of the cells}

Cells were fixed in a $4 \%$ solution of paraformaldehyde (PFA) in PBS for 20 minutes. After fixation, cells were washed in PBS, mounted with ProLong Gold and allowed to dry overnight. The next day the slides were sealed with nail varnish. 


\subsection{Cytospin}

HEK-293 cells expressing GFP-LC3 were centrifuged onto polylysine treated slides (PolyPrep slides) using a Cytospin centrifuge. This was necessary because HEK-293 cells grow one on top of another, therefore making it difficult to quantify the cells or structures within them. For fixation on the slides, cells were collected, centrifuged and resuspended in PBS aiming at a concentration of 400.000 cells $/ \mathrm{ml}$. Approximately $100 \mu \mathrm{l}$ of this solution were used for loading into the Cytospin. Cells were centrifuged for 2 minutes at $1000 \mathrm{rpm}$. Subsequently, the samples were treated with $4 \%$ PFA as above and a coverslip with a drop of ProLong Gold was put over them. The next day the samples were sealed with nail varnish.

\subsection{GFP-LC3 punctae analysis}

For punctae quantification the slides were blinded, representative images of the sample were taken and the punctae of at least 70 cells per sample were counted from the amplified images. For these analyses a Zeiss Axioplan fluorescence microscope with the 63 *objective (oil) was used. The confocal images were taken with a Leica SP5 confocal microscope at the CIC Salamanca. The GFP was visualised using an argon laser at $488 \mathrm{~nm}$.

\subsection{Surface stainings}

In order to stain transfected or endogenous AIR on the cell surface the cells were seeded onto coverslips which had been treated with poly-L-lysine. To prevent endocytosis in this type of staining, the cells were kept at $4^{\circ} \mathrm{C}$ and in sodium azide $(0.1 \%)$ and BSA $(2 \%)$ containing PBS from the moment they were removed from the cell culture dish. The same solution was used for washing the cells after antibody incubation. The to-be-stained cells had been either seeded out $24 \mathrm{~h}$ earlier (SAOS-2) or, in the case of the HEK-293 cells, transfected $36 \mathrm{~h}$ before. The cells were removed from the cell culture medium, washed twice with $\mathrm{NaN}_{3} / \mathrm{BSA} / \mathrm{PBS}$ and incubated for 1 hour at $4{ }^{\circ} \mathrm{C}$ with a rabbit anti-AIR antibody $(1 / 100)$ or for the HA-tagged version of AIR with an anti-HA antibody $(1 / 500)$. Afterwards the cells were washed three times and incubated in the dark for 1 hour at $4{ }^{\circ} \mathrm{C}$ with a Cy3-coupled anti-rabbit secondary antibody (1/400) for the anti-AIR antibody or an anti-mouse alexa-coupled secondary antibody (1/400) for the HA tag. Once the secondary antibody had been washed off ( 3 times with $\mathrm{NaN}_{3}$ /BSA/PBS), the cells were fixed with paraformaldehyde (see 10.1.) and the nuclei stained with $1 \mu \mathrm{g} / \mathrm{ml} \operatorname{DAPI}\left(4^{\prime}, 6^{\prime}\right.$ 
diamidino-2-phenylindole) in PBS during 3 minutes. Subsequently, the cells were washed and mounted as described above (10.1.). The confocal images were taken with the Leica SP5 confocal microscope at the CIC Salamanca using the helium-neon laser at $543 \mathrm{~nm}$ for $\mathrm{Cy} 3$ or the argon laser at $458 \mathrm{~nm}$ for the alexa fluorophore.

\subsection{Double stainings}

For the AIR localisation studies, SAOS-2 cells were stained with the respective marker and AIR at one time. The cells had been seeded out on coverslips and were fixed with paraformaldehyde prior to cell permeabilisation and blocking of free aldehyde groups for 30 minutes with $0.5 \%$ Igepal CA-630 detergent (Sigma-Aldrich) and 1\% glycine (USB Affymetrix). Before incubating the cells with the rabbit anti-AIR antibody $(1 / 100)$ together with anti-CD63 (1/20), anti-GM130 (1/300) or anti-Lamp2 $(1 / 15)$ in $2 \%$ BSA/PBS, the samples were left in $3 \%$ BSA/PBS during 30 minutes for blocking. Antibody incubations took place at room temperature and in the dark for 1 hour. Subsequently, the cells were washed three times with PBS and incubated with the secondary antibodies mixture, anti-mouse coupled Су $3(1 / 400)$ and anti-rabbit coupled Alexa (1/400) for 1 hour. Cells were washed with PBS again, stained with DAPI and mounted for confocal microscopy (see above). The confocal images were taken with the Leica SP5 confocal microscope at the CIC Salamanca using the helium-neon laser at $543 \mathrm{~nm}$ for Cy 3 and the argon laser at $458 \mathrm{~nm}$ for the alexa fluorophore. In order to avoid overspilling signals from the samples, the lasers were activated one at a time and not simultaneously.

\section{Flow cytometry}

To measure the expression levels of the different chimera on the cell surface, a FITC (5(6)-fluorescein isothiocyanate mixed isomer)-coupled anti-CD16 antibody was used. In order to prevent endocytosis of the chimera the cells were kept in ice and in the presence of sodium azide $(0.1 \%)$. HEK-293 in 24 -well plates were transfected with $0.5 \mu \mathrm{g}$ of the plasmid encoding for the relevant chimera and $0.5 \mu \mathrm{g}$ P12-Poly. 40 hours after transfection, the cells were collected by pipetting and centrifuged for $5 \mathrm{~min}$ at $600 \mathrm{~g}$ and $4{ }^{\circ} \mathrm{C}$. After that they were suspended in $90 \mu \mathrm{l} 2 \% \mathrm{BSA} / 0.1 \% \mathrm{NaN}_{3} / \mathrm{PBS}$ with $15 \mu \mathrm{l} \mathrm{FITC}$ coupled CD16-antibody and incubated in ice and in the dark for $1 \mathrm{~h}$. The tubes were tapped every 10 minutes to resuspend the cells. Before passing the cells through the cytometer (FACS Calibur E 1738) they were washed three times by adding $3 \mathrm{ml} 2 \%$ BSA/0.1\% $\mathrm{NaN}_{3} / \mathrm{PBS}$, centrifuging for 3 minutes at $600 \mathrm{~g}_{\mathrm{n}}$ and $4{ }^{\circ} \mathrm{C}$ and taking off the supernatant with a vacuum pump. The measured parameter was the F1 fluorescence. 
Samples were compared by their medium fluorescence intensity and the percentage of the cells showing positive for green fluorescence. The software used for recording and analysing the data was Cell Quest. 



\section{Abbreviations}

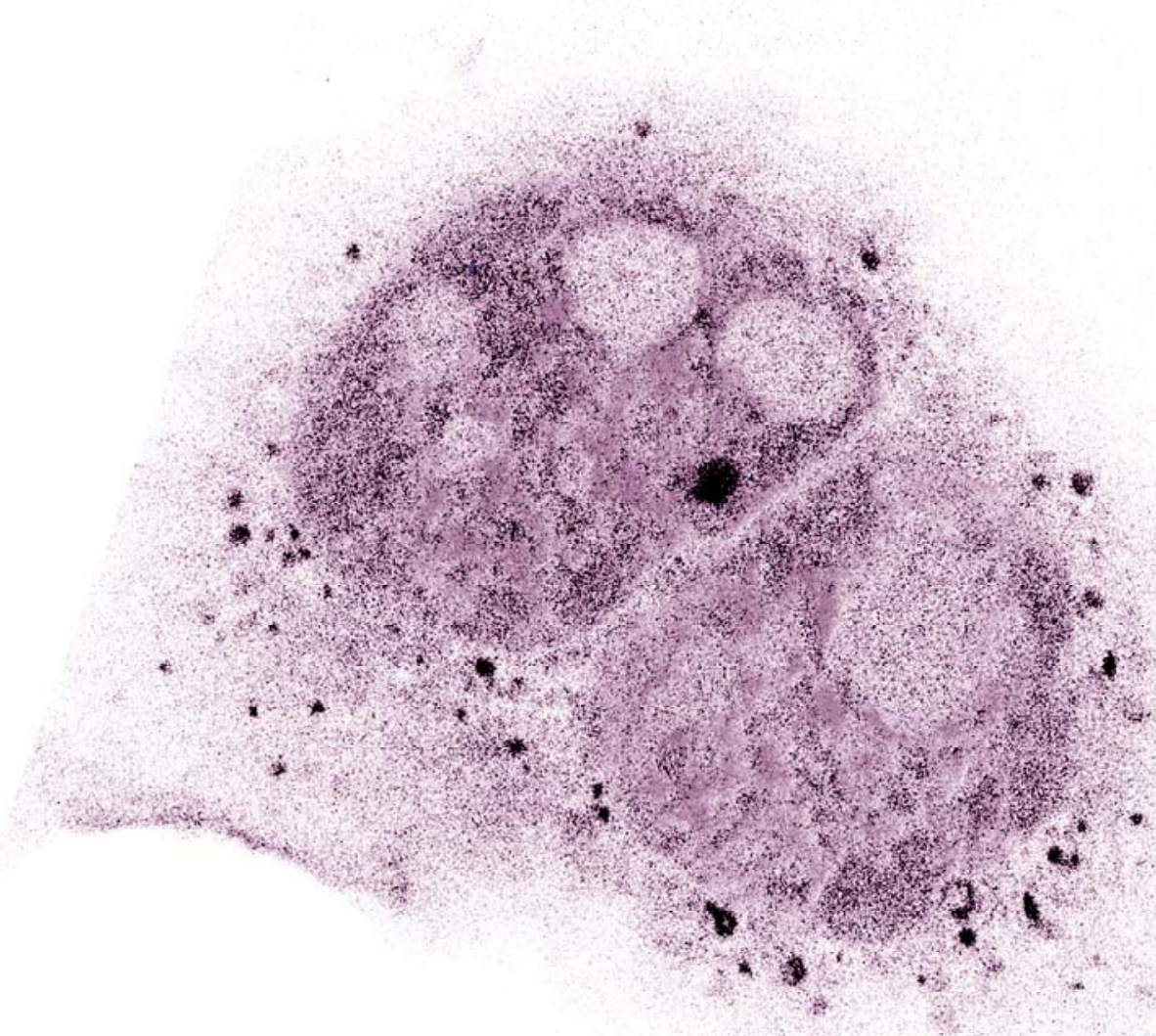





\begin{tabular}{|c|c|}
\hline \multicolumn{2}{|c|}{ Abbreviation } \\
\hline 3-MA & 3-Methyladenine \\
\hline 4E-BP1 & Eukaryotic initiation factor 4E binding protein 1 \\
\hline aa & Amino acid \\
\hline$A D$ & Alzheimer's disease \\
\hline AIR & Autophagy inducing receptor \\
\hline Ambra1 & Activating molecule in Beclin1-regulated autophagy \\
\hline AMP & Adenosin monophosphate \\
\hline AMPK & Adenosin monophophate activated protein kinase \\
\hline Ams1 & a-mannosidase \\
\hline Ape1 & Aminopeptidase I \\
\hline APP & Amyloid beta precursor protein \\
\hline Atg & Autophagy (related gene) \\
\hline Atg16L & Atg16-like \\
\hline ATP & Adenosin triphophate \\
\hline$A \beta$ & Amyloidogenic A beta \\
\hline Bak & $\mathrm{Bcl}-2$ homologous antagonist killer \\
\hline Bax & Bcl-2-associated $X$ protein \\
\hline BBD & Bcl-2-binding domain \\
\hline $\mathrm{Bcl}-2$ & B-cell lymphoma 2 \\
\hline $\mathrm{Bcl}-\mathrm{xl}$ & Bcl-X long \\
\hline $\mathrm{BH}$ & Bcl-2 homolgy \\
\hline Bid & $\mathrm{BH} 3$ interacting domain death agonist \\
\hline Bif-1 & Bax-interacting factor 1 \\
\hline Bik & $\mathrm{Bcl}-2$ interacting killer \\
\hline Bim & $\mathrm{Bcl}-2$ interacting mediator of cell death \\
\hline BNIP3 & $\mathrm{Bcl}-2$ /adenovirus E1B 19-kDa-interacting protein 3 \\
\hline BNIP3L & BNIP3 like \\
\hline BSA & Bovine standard albumin \\
\hline BSMAP & Brain-specific membrane anchored protein \\
\hline C & Celsius \\
\hline C1orf8 & C1 open reading frame 8 \\
\hline CaMKK- $\beta$ & $\mathrm{Ca}^{2+} /$ calmodulin-dependent kinase $\beta$ \\
\hline CCD & Central coiled-coil domain \\
\hline
\end{tabular}




\begin{tabular}{|c|c|}
\hline CD4 & Cluster of differentiation 4 \\
\hline cDNA & Copy DNA \\
\hline $\mathrm{CIC}$ & $\begin{array}{l}\text { Centro de Investigación del Cáncer (Centre of Cancer Research), } \\
\text { Salamanca }\end{array}$ \\
\hline CMA & Chaperone mediated autophagy \\
\hline Cos & CV-1 in origin and carrying the SV4O genetic material \\
\hline Cvt & Cytoplasm to the vacuole targeting pathwaty \\
\hline DAPI & 4',6' diamidino-2-phenylindole \\
\hline DAPK & Death-associated protein kinase \\
\hline DISC & Death inducing signalling complex \\
\hline DMEM & Dulbecco's modified Eagle's medium \\
\hline DMSO & Dimethylsulfoxide \\
\hline DNA & Desoxyribonucleic acid \\
\hline dNTP & Deoxynucleotide triphosphate \\
\hline DRAM & Damage regulated autophagy modulator \\
\hline DTT & Dithiothreitol \\
\hline ECD & Evolutionarily conserved domain \\
\hline ECL & Enhanced chemiluminescence \\
\hline EDTA & Ethylenediaminetetraacetic acid \\
\hline elF4E & Eukaryotic initiation factor $4 \mathrm{E}$ \\
\hline ES (cell) & Embryonic stem (cell) \\
\hline f. c. & Final concentration \\
\hline FACS & Fluorescence activated cell sorting \\
\hline FBS & Foetal bovine serum \\
\hline FcyR & Fc gamma receptor \\
\hline FIP200 & Focal adhesion kinase family-interacting protein of $200 \mathrm{kDa}$ \\
\hline FITC & Fluorescein isothiacyanate \\
\hline FL & Full length \\
\hline FoxO3 & Forkhead box $\mathrm{O3}$ \\
\hline g & Gram \\
\hline GFP & Green fluorescent protein \\
\hline$g_{n}$ & Gravitational force $\left(9.81 \mathrm{~m} / \mathrm{s}^{2}\right)$ \\
\hline GSH & Glutathione \\
\hline GST & Glutathione-S-transferase \\
\hline GTPase & Guanosine triphosphate hydrolase \\
\hline
\end{tabular}




\begin{tabular}{|c|c|}
\hline $\mathrm{G} \beta \mathrm{L}(=\mathrm{mLST} 8)$ & Mammalian G-protein $\beta$-subunit like protein \\
\hline $\mathrm{h}$ & Hour(s) \\
\hline $\mathrm{HA}$ & Epitope sequence hemagglutinin: YPYDVPDYA \\
\hline НCT-116 & Human colorectal carcinoma - 116 \\
\hline HD & Huntington's disease \\
\hline HEK-293 & Human embryonic kidney cells - 293 \\
\hline HeLa & Cells from Henrietta Lack's cervical cancer tumour \\
\hline HER-2 & Human Epidermal growth factor Receptor 2 \\
\hline Hif & Hypoxia induced factor \\
\hline HIV & Human immunodeficiency virus \\
\hline HRP & Horseradish peroxidase \\
\hline Hrs & Hepatocyte growth factor-regulated tyrosine kinase substrate \\
\hline $\mathrm{Hsc70}$ & Heat shock cognate protein of $70 \mathrm{kDa}$ \\
\hline HSV & Herpes simplex virus \\
\hline $\mathrm{Htt}$ & Huntingtin protein \\
\hline ID & Intracellular domain \\
\hline IF & Immunofluorescence \\
\hline IFN & Interferon \\
\hline $\lg$ & Immunoglobulin \\
\hline IL & Interleukin \\
\hline IP & Immunoprecipitation \\
\hline IPAF & Interleukin-1beta converting enzyme (ICE) protease-activating factor \\
\hline IRES & Internal ribosome entry site \\
\hline IRG & Immunity regulated GTPase \\
\hline JNK1 & c-Jun N-terminal protein kinase \\
\hline $\mathrm{kbp}$ & Kilo-base-pair \\
\hline $\mathrm{kDa}$ & Kilodalton \\
\hline I & Litre \\
\hline LAMP & Lysosome associated membrane protein \\
\hline LB & Luria-Bertani broth \\
\hline LC3 & Microtubule associated protein 1-light chain 3 \\
\hline LPS & Lipopolysaccharide \\
\hline LRR & Leucine rich repeat \\
\hline M & Mol/Litre \\
\hline $\mathrm{m}$ & Milli- \\
\hline
\end{tabular}




\begin{tabular}{|c|c|}
\hline m & Meter \\
\hline MAPK & Mitogen-activated protein kinase \\
\hline Mcl1 & Myeloid leukemia cell differentiation protein \\
\hline MEF & Mouse embryonic fibroblast \\
\hline $\mathrm{MHC}$ & Major histocompatibility complex \\
\hline $\min$ & Minutes \\
\hline $\mathrm{mLST} 8$ / G $\beta \mathrm{L}$ & Mammalian G-protein $\beta$-subunit like protein \\
\hline mol & Mol \\
\hline mSin1 & Mammalian stress-activated protein kinase-interacting protein 1 \\
\hline m-TOR & Mammalian target of rapamycin \\
\hline mTORC1 / & Mammalian target of rapamycin complex 1 or 2 \\
\hline mTORC2 & \\
\hline MWCO & Molecular weight cut-off \\
\hline MyD88 & Myeloid differentiation primary response gene 88 \\
\hline $\mathrm{n}$ & Nano- \\
\hline NAIP & Neuronal apoptosis inhibitory protein \\
\hline NALP & NACHT domain, leucine-rich repeat-, and PYD-containing proteins \\
\hline NFkB & Nuclear factor kappa B \\
\hline NLR & Nucleotide-binding-and-oligomerization-domain-like receptor \\
\hline Nod1/Nod2 & Nucleotide-binding and oligomerization domain receptor (1 or 2 ) \\
\hline $\mathrm{P} 12$ & pEAK12 \\
\hline P8 & pEAK8 \\
\hline PAGE & Polyacrylamide gel electrophoresis \\
\hline PAMP & Pathogen associated molecular pattern \\
\hline PAS & Phagophore assembly site \\
\hline PBS & Phosphate buffered saline \\
\hline PCD & Programmed cell death \\
\hline PCR & Polymerase chain reaction \\
\hline PD & Parkinson's disease \\
\hline $\mathrm{pDC}$ & Plasmacytoid dentritic cell \\
\hline PE & Phosphatidylethanolamine \\
\hline PFA & Paraformaldehyde \\
\hline PGN & Peptidoglycan \\
\hline $\mathrm{PI}$ & Propidium iodide \\
\hline PI3K & Phosphatidylinositol-3-kinase \\
\hline
\end{tabular}




\begin{tabular}{|c|c|}
\hline PKB & Protein kinase $B$ \\
\hline PMN & Piecemeal microautophagy of the nucleus \\
\hline PMSF & Phenylmethyl sulphonyl fluoride \\
\hline PRAS40 & Proline-rich Akt substrate $40-\mathrm{kDa}$ \\
\hline PRR & Pattern recognition receptors \\
\hline PS-1 & Presenilin 1 \\
\hline Ptdlns3P & Phophatidylinositol-tri-phophate \\
\hline PTEN & Phosphatase and tensin homolog \\
\hline Puma & P53-upregulated modulator of apoptosis \\
\hline Raptor & Regulatory associated protein of mTOR \\
\hline Rictor & Rapamycin-insensitive companion of mTOR \\
\hline RIG-I & Retinoic acid-inducible gene I \\
\hline RIP & Receptor interacting protein kinase \\
\hline RLH & Retinoic acid-inducible gene I-like helicase \\
\hline RNA & Ribonucleic acid \\
\hline ROS & Reactive oxygen species \\
\hline $\mathrm{rpm}$ & Rounds per minute \\
\hline S6K1 & S6 kinase 1 \\
\hline SAOS-2 & Sarcoma osteogenic-2 \\
\hline SCV & Salmonella containing vacuoles \\
\hline SDS & Sodium dodecyl sulfate \\
\hline siRNA & Small inhibiting RNA \\
\hline SMAC/DIABLO & $\begin{array}{l}\text { Second mitochondria-derived activator of caspases/direct inhibitor of } \\
\text { apoptosis-binding protein with low pl }\end{array}$ \\
\hline SQSTM1 (=p62) & Sequestosome-1 \\
\hline ssRNA & Single stranded ribonucleic acid \\
\hline STAT & Signal Transducer and Activator of Transcription \\
\hline TBS & Tris-buffered saline \\
\hline TE & Tris/EDTA \\
\hline TEC & Thymic epithelial cell \\
\hline Th & T-helper (cell type) \\
\hline TLR & Toll-like receptor \\
\hline Tmem59 & Transmembrane protein 59 \\
\hline TNF & Tumour necrosis factor \\
\hline TOR & Target of rapamycin \\
\hline
\end{tabular}




\begin{tabular}{|ll|}
\hline TRIF & Toll/IL-1 receptor domain-containing adaptor inducing IFN- $\beta$ \\
TRIS & Tris(hydroxymethyl)aminomethan \\
TSC & Tuberous sclerosis complex \\
U & Unit \\
UV & Ultraviolet \\
UVRAG & UV-radiation resistance-associated gene protein \\
VMP1 & Vacuole membrane protein 1 \\
Vps & Vacuolar protein sorting \\
WB & Western blot(ting) \\
WM & Wortmannin \\
WT & Wild type \\
Z-VAD-fmk & Carbobenzoxy-valyl-alanyl-aspartyl-[O-methyl]- fluoromethylketone \\
$\mu$ & Micro- \\
\hline
\end{tabular}




\section{Materials-Tables}

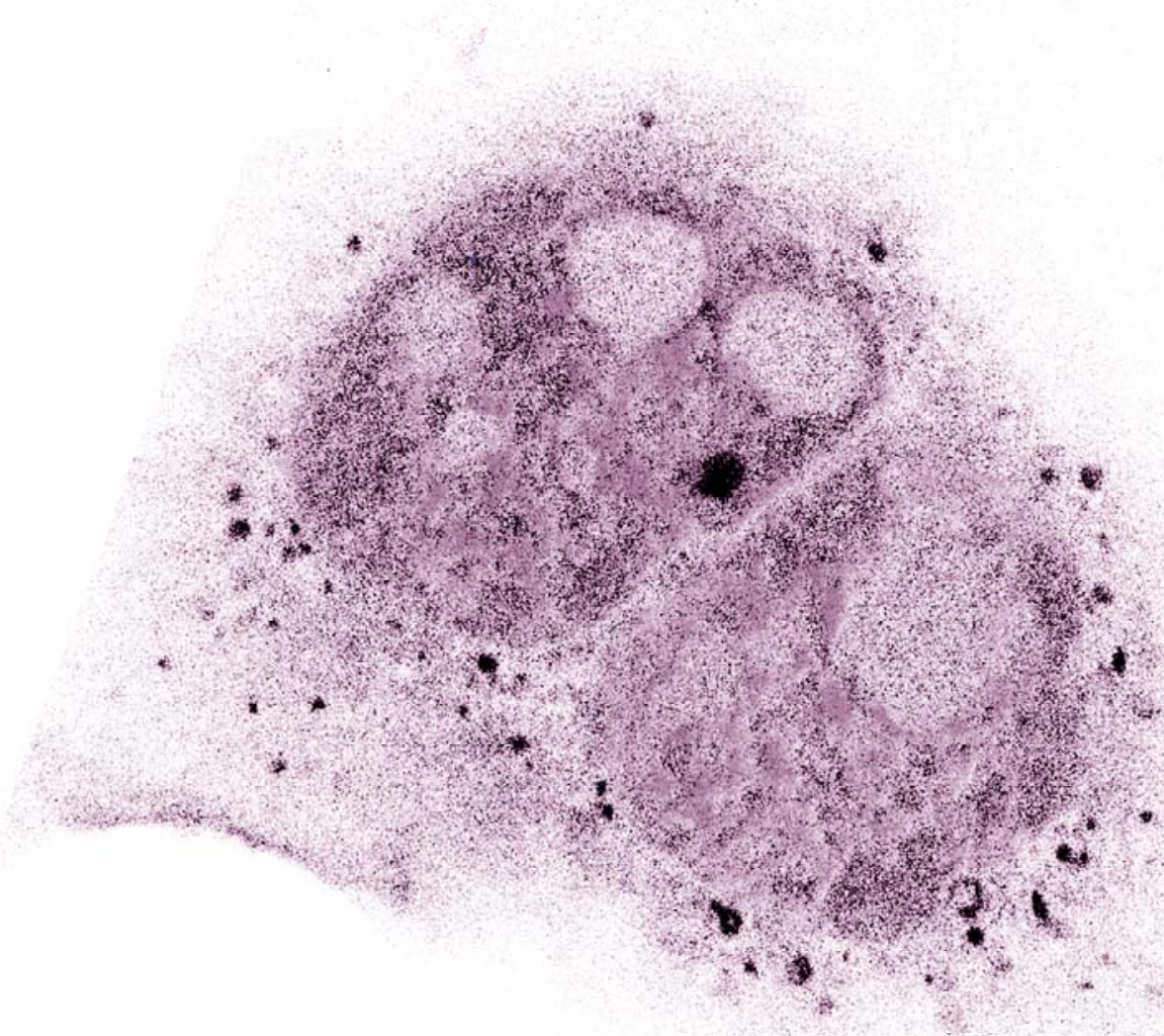



MATERIALS-TABLES

Table 1: Chemicals, enzymes and other commercially acquired material

Chemicals, enzymes and other laboratory material

Product

DNA handling

Agarose D-1 low EEO (electroendoosmosis)

Nu Sieve ${ }^{\circledR}$ GTG® Agarose

Ethidium bromide

$1 \mathrm{~kb}$ ladder marker

Adenin triphosphate (ATP)

Ampicillin

Calf intestinal phosphate (CIP) $10000 \mathrm{U} / \mathrm{ml}$

DNA polymerization mix dNTP Set $(A, C, G, T)$

Eco R I $10000 \mathrm{U} / \mathrm{ml}$

Hind III $10000 \mathrm{U} / \mathrm{ml}$

Mlu I $10000 \mathrm{U} / \mathrm{ml}$

Not I $10000 \mathrm{U} / \mathrm{ml}$

Pci I $10000 \mathrm{U} / \mathrm{ml}$

Pfu buffer 10*

Pfu DNA polymerase $3000 \mathrm{U} / \mathrm{ml}$

Polymerase kinase (PNK) $10000 \mathrm{U} / \mathrm{ml}$

Polymerase kinase (PNK) buffer 10*

Spe I $10000 \mathrm{U} / \mathrm{ml}$

T4 DNA ligase $400000 \mathrm{U} / \mathrm{ml}$

T4 DNA ligase buffer with 10mM ATP
Provider

Use

Pronadisa
Cambrex
Sigma
New England BioLabs
Roche
Calbiochem
New England BioLabs
GE Healthcare

New England BioLabs

New England BioLabs

New England BioLabs

New England BioLabs

New England BioLabs

Promega

Promega

New England BioLabs

New England BioLabs

New England BioLabs

New England BioLabs

New England BioLabs
DNA electrophoresis

DNA electrophoresis

DNA electrophoresis

DNA electrophoresis

Coenzyme for kinase

Bacteria growth

Phosphatase

Deoxynucleotides for polymerase

Restriction enzyme

Restriction enzyme

Restriction enzyme

Restriction enzyme

Restriction enzyme

Polymerase buffer

Polymerase

Kinase

Kinase buffer

Restriction enzyme

Ligase

Ligase buffer 
MATERIALS-TABLES

\begin{tabular}{|c|c|c|}
\hline QIA quick PCR purification kit & Qiagen & DNA purification \\
\hline QIA prep spin miniprep kit & Qiagen & DNA purification \\
\hline QIA prep plasmid midi kit & Qiagen & DNA purification \\
\hline QIA prep plasmid maxi kit & Qiagen & DNA purification \\
\hline \multicolumn{3}{|l|}{ Antibody production } \\
\hline AminoLink plus Immo kit & Pierce & Antibody purification \\
\hline Dithiothreitol (DTT) & Sigma & Reducing agent \\
\hline Eggcellent chicken IgY kit & Pierce & Antibody purification \\
\hline Glycine & USB-Affymetrix & Antibody purification \\
\hline Immo GST column & Pierce & Antibody purification \\
\hline Lysozyme & Sigma & Bacteria lysis \\
\hline $\mathrm{N}$-laurylsarcosine sodium salt (Sarkosyl) & Sigma & Bacteria lysis \\
\hline Protein G Sepharose 4 Fast Flow & GE Healthcare & Antibody purification \\
\hline Protran BA 85 nitrocellulose & Whatman & Antibody purification \\
\hline Slide-A-Lyzer, dialysis cassette & Pierce & Antibody purification \\
\hline Triton $\mathrm{X}-100$ & Sigma & Bacteria lysis \\
\hline \multicolumn{3}{|l|}{ Protein handling } \\
\hline Albumin standard $2 \mathrm{mg} / \mathrm{ml}$ & Thermo Scientific & Protein measuring \\
\hline Ammoniumpersulfate & Sigma & SDS-Page gels \\
\hline Aprotinin & Roche & Protease inhibitor \\
\hline BioRad Dc protein assay & Bio Rad & Protein measuring \\
\hline Bovine standard albumin (BSA) fraction $V$ & Sigma & Antibody solutions \\
\hline Bromphenol blue & Sigma & SDS-Page sample buffer \\
\hline Guanidine & Sigma & Membrane stripping \\
\hline Igepal CA-360 & Sigma & Detergent for cell lysis \\
\hline Immobilion $\mathrm{P}$ transfer membrane & Millipore & Immunoblotting \\
\hline
\end{tabular}


MATERIALS-TABLES

\begin{tabular}{|c|c|c|}
\hline $\mathrm{N}, \mathrm{N}, \mathrm{N}, \mathrm{N}-$ Tetramethylethylendiamine & Sigma & SDS-Page gels \\
\hline Non-fat dried milk powder & Nestle Sveltesse & Membrane blocking \\
\hline Pepstatin & Roche & Protease inhibitor \\
\hline PMSF (phenylmethylsulfonyl fluoride) & Sigma & Protease inhibitor \\
\hline Rainbow high range molecular weight marker & GE Healthcare & SDS-Page \\
\hline Sodium azide & Sigma & Antibody solutions \\
\hline Tween 20 & Sigma & Membrane washing \\
\hline$\beta$-Mercaptoethanol & Fluka & SDS-Page sample buffer \\
\hline \multicolumn{3}{|l|}{ Microscopy and flow cytometry } \\
\hline DAPI (4;,6' diamidino-2-phenylindole) & Roche & Cell microscopy \\
\hline Facs Flow & $\mathrm{BD}$ & Flow cytometry \\
\hline Microscope slides & Linea Lab & Cell microscopy \\
\hline Paraformaldehyde & Sigma & Cell microscopy \\
\hline Prolong Gold antifade reagent & Invitrogen & Cell microscopy \\
\hline \multicolumn{3}{|l|}{ Tissue culture } \\
\hline $0.2 \mu \mathrm{m}$ filter $\mathrm{FP} 30 / 0.2$ cellulose acetate & Schleicher \& Schuell & Sterile filtration \\
\hline 3-Methyladenin & Sigma & Autophagy inhibitor \\
\hline Calcium chloride, tissue culture grade & Sigma & Transfections \\
\hline Chloroquine phosphate & Sigma & Endocytosis inhibitor \\
\hline Coverslips & Thermo scientific & Cell microscopy \\
\hline Dimethyl sulfoxide, Hybri-Max, sterile-filtered & Sigma & Cell storage, solvent \\
\hline Dulbecco's Modified Eagle Medium; 4.5g Glucose $/ \mathrm{L} ; 0.11 \mathrm{~g} / \mathrm{L}$ sodium pyruvate & Gibco & Cell culture \\
\hline Fetal bovine serum & Gibco & Cell culture \\
\hline Glutamine $200 \mathrm{mM} 100 *$, tissue culture grade & Gibco & Cell culture \\
\hline JetPEI & PolyPlus Transfection & Transfection agent \\
\hline
\end{tabular}


MATERIALS-TABLES

Penicillin $10000 \mathrm{U} / \mathrm{ml}$; Streptomycin $10000 \mu \mathrm{g} / \mathrm{ml}$, tissue culture grade

Gibco

Cell culture

Poly-L-lysine $0.01 \%$ molecular weight 150000 - 300 000, tissue culture grade

Sigma

Coverslip treatment

Poly-Prep-Slides

Sigma

Cell microscopy

Sodium chloride solution, biotech-performance certified $5 \mathrm{M}$

Sigma

Sterile needles

BD Plastipack

Transfections

Sterile plastic pipettes

Falcon

Sterile syringes

Cell culture

Tissue culture plates

BD Plastipack

Cell culture

Trypsin-EDTA $0.25 \%$, tissue culture grade

Falcon

Cell culture

Gibco

Cell culture

Wortmannin

Sigma

Cell culture

Table 2: Buffers prepared by the CIC sterilization unit, Salamanca or in the laboratory

Non-commercially acquired buffers

Buffer

Ingredients

Sterile

Buffers which were prepared by the Centre of Cancer Research sterilization unit, Salamanca

\begin{tabular}{|c|c|c|}
\hline 0.5M EDTA pH 8.0 & Ethylenediaminetetraacetic acid $0.5 \mathrm{~mol} / \mathrm{l} ; \mathrm{pH}$ adjusted with sodium hydroxide & yes \\
\hline $80 \%$ Glycerol & $80 \%$ Gycerol & yes \\
\hline LB (Luria Bertani medium) & $0.5 \%$ yeast extract; $1 \%$ sodium chloride; $1 \%$ tryptone peptides & yes \\
\hline LB Agar & $0.5 \%$ yeast extract; $1 \%$ sodium chloride; $1 \%$ tryptone peptides; $2 \%$ agar & yes \\
\hline LB Agar culture dish & LB Agar with $100 \mathrm{mg} / \mathrm{ml}$ ampicillin & yes \\
\hline Lower Tris pH 8.8 & 1.5 M Tris; $14 \mathrm{mM}$ SDS (sodium dodecyl sulfate); $\mathrm{pH}$ adjusted with hydrochloric acid & no \\
\hline $\begin{array}{l}\text { PBS (Phosphate buffered } \\
\text { saline) }\end{array}$ & $2.68 \mathrm{mM} \mathrm{KCl} ; 1.47 \mathrm{mM} \mathrm{KH} 2 \mathrm{PO} 4 ; 137 \mathrm{mM} \mathrm{NaCl} ; 7.98 \mathrm{mM} \mathrm{Na} 2 \mathrm{HPO} 4$ & yes \\
\hline PBS $10 *$ & $26.8 \mathrm{mM} \mathrm{KCl} ; 14.7 \mathrm{mM} \mathrm{KH} 2 \mathrm{PO} 4 ; 1370 \mathrm{mM} \mathrm{NaCl} ; 79.8 \mathrm{mM} \mathrm{Na} 2 \mathrm{HPO} 4$ & yes \\
\hline SDS $10 \%$ & Sodium dodecyl sulfate $10 \%$ & no \\
\hline
\end{tabular}




\section{MATERIALS-TABLES}

SDS-PAGE $10 *$

$0.25 \mathrm{M}$ Tris; $2 \mathrm{M}$ glycine; $0.5 \%$ SDS

no

TAE 50* $\mathrm{pH} 8.0$

2M Tris; 0.05M EDTA; $\mathrm{pH}$ adjusted with acetate

yes

TBS 20* $\mathrm{pH} 8.0$

$0.5 \mathrm{M}$ Tris; $1 \mathrm{M} \mathrm{NaCl} ; 0.05 \mathrm{KCl} ; \mathrm{pH}$ adjusted with hydrochloric acid

TE

10mM Tris; 1mM EDTA

Transfer buffer $10 *$ $0.25 \mathrm{M}$ Tris; $1.92 \mathrm{M}$ glycine

Tris $1 \mathrm{M}$ at various $\mathrm{pH}$

$1 \mathrm{M}$ Tris; $\mathrm{pH}$ adjusted with hydrochloric acid

Upper TRIS pH 8.5

$0.5 \mathrm{M}$ Tris; $14 \mathrm{mM}$ SDS; $\mathrm{pH}$ adjusted with hydrochloric acid

no

\section{Buffers which were prepared in the laboratory}

\begin{tabular}{llll} 
HEBS pH 7.1 & $280 \mathrm{mM} \mathrm{NaCl} ; 1.5 \mathrm{mM} \mathrm{Na}_{2} \mathrm{HPO}_{4} ; 11 \mathrm{mM}$ glucose and 50mM HEPES (Sigma) & yes & \\
\hline Loading buffer pH 6.8 & $100 \mathrm{mM}$ Tris; $10 \%$ ß-mercaptoethanol; $4 \%$ SDS; $0.2 \%$ bromphenol blue; $20 \%$ glycerol & no & no \\
\hline Lysis buffer pH 7.5 & $50 \mathrm{mM}$ Tris; $150 \mathrm{mM} \mathrm{NaCl} ; 5 \mathrm{mM}$ EDTA; $1 \%[\mathrm{v} / \mathrm{v}]$ Igepal CA-360 & no \\
NET pH 8.0 & $20 \mathrm{mM}$ Tris; $150 \mathrm{mM} \mathrm{NaCl;} 1 \mathrm{mM}$ EDTA & no \\
STE pH 8.0 & $10 \mathrm{mM}$ Tris; $50 \mathrm{mM} \mathrm{NaCl} ; 1 \mathrm{mM}$ EDTA &
\end{tabular}

\section{Table 3: Primary antibodies}

\begin{tabular}{|c|c|c|c|c|c|c|c|c|}
\hline \multicolumn{2}{|c|}{ Primary antibodies } & \multirow[b]{2}{*}{ Type (clone) } & \multirow[b]{2}{*}{ Provider } & \multirow[b]{2}{*}{ Catalogue No } & \multirow[b]{2}{*}{ Application } & \multirow[b]{2}{*}{ Working conc. } & \multirow[b]{2}{*}{ Dilution } & \multirow[b]{2}{*}{ Target molecular weight } \\
\hline Antibody & Host & & & & & & & \\
\hline$\alpha-A c t i n$ & mouse & monoclonal (AC40) & Sigma & A 3853 & Western blotting & not known & $1 / 1800-1 / 1000$ & $42 \mathrm{kDa}$ \\
\hline$\alpha-A I R$ & rabbit & polyclonal & Laboratory & & Western blotting & not known & $1 / 6500$ & $36 \mathrm{kDa}$ \\
\hline$\alpha-\operatorname{Atg} 5$ & rabbit & $\begin{array}{l}\text { polyclonal } \\
\text { C-terminal }\end{array}$ & Sigma & AO731 & Western blotting & not known & $1 / 750$ & 56 kDa \\
\hline$\alpha-\operatorname{Atg} 7$ & rabbit & polyclonal & Cell Signaling & 2631 & Western blotting & not known & $1 / 1000$ & 78 kDa \\
\hline$\alpha-$ Beclin & mouse & monoclonal & BD Pharmigen & 612112 & Western lotting & $0.25 \mu \mathrm{g} / \mathrm{ml}$ & $1 / 750$ & $61 \mathrm{kDa}$ \\
\hline$\alpha-C D 16$ & mouse & $\begin{array}{l}\text { monoclonal } \\
\text { (DJ 130c) }\end{array}$ & Santa Cruz & sc-20052 & Western blotting & $0.4 \mu \mathrm{g} / \mathrm{ml}$ & $1 / 500$ & $50-100$ kDa \\
\hline
\end{tabular}


MATERIALS-TABLES

\begin{tabular}{|c|c|c|c|c|c|c|c|c|}
\hline$\alpha$-GST & mouse & monoclonal (B14) & Santa Cruz & sc-138 & Western blotting & $1 \mu \mathrm{g} / \mathrm{ml}$ & $1 / 200$ & $26 \mathrm{kDa}$ \\
\hline$\alpha-H A .11$ & mouse & monoclonal (16B12) & Covance & AFC-101P & Western blotting & not known & $1 / 1000$ & tagged protein \\
\hline $\begin{array}{l}\alpha-C D 16 \text { FITC- } \\
\text { coupled }\end{array}$ & mouse & monoclonal & BD Pharmigen & 555406 & Flow cytometry & not known & $1 / 6$ & \\
\hline a-CD63 LIMP & mouse & monoclonal & $\begin{array}{l}\text { University lowa } \\
\text { hybridoma bank }\end{array}$ & $\mathrm{H} 5 \mathrm{C} 6$ & Immunofluorescence & & $1 / 20$ & \\
\hline a-Lamp2 & mouse & monoclonal & $\begin{array}{l}\text { University lowa } \\
\text { hybridoma bank }\end{array}$ & H4B 4 & Immunofluorescence & & $1 / 15$ & \\
\hline a-GM130 & mouse & monoclonal & BD Pharmigen & 610822 & Immunofluorescence & $0.83 \mu \mathrm{g} / \mathrm{ml}$ & $1 / 300$ & \\
\hline
\end{tabular}

Table 4: Secondary antibodies

\begin{tabular}{|c|c|c|c|c|c|c|c|}
\hline Secondary antibodies & & & & & & & \\
\hline Antibody & Host & Conjugated with & Company & Catologue No & Application & Working conc. & Dilution \\
\hline$\alpha-r a b b i t$ & goat & horse raddish peroxidase & Dako & P0448 & Western blotting & & $1 / 5000$ \\
\hline$\alpha$-chicken & goat & horse raddish peroxidase & Abcam & $a b 97131$ & Western blotting & & $1 / 20000$ \\
\hline$\alpha$-mouse for tissue culture & rabbit & & $\begin{array}{l}\text { Jackson Immuno } \\
\text { Research }\end{array}$ & $315-005-008$ & artificial ligand & $10 \mu \mathrm{g} / \mathrm{ml}$ & $1 / 240$ \\
\hline$\alpha$-mouse & goat & Суз & $\begin{array}{l}\text { Jackson Immuno } \\
\text { Research }\end{array}$ & $111-165-003$ & Immunofluorescence & & $1 / 400$ \\
\hline$\alpha$-rabbit & goat & Суз & $\begin{array}{l}\text { Jackson Immuno } \\
\text { Research }\end{array}$ & $115-165-003$ & Immunofluorescence & & $1 / 400$ \\
\hline$\alpha$-mouse & goat & Alexa-Fluor 488 & Invitrogen & A-11070 & Immunofluorescence & & $1 / 400$ \\
\hline$\alpha-$ rabbit & rabbit & Alexa-Fluor 488 & Invitrogen & $A-11059$ & Immunofluorescence & & $1 / 400$ \\
\hline
\end{tabular}




\section{MATERIALS-TABLES}

Table 5: Cell lines used

Cells

Name

Source

Human cell lines

$\begin{array}{ll}\text { HCT } 116 & \text { Dr. Bert Vogelstein (Baltimore) } \\ \text { HEK-293/ HEK-293T } & \text { American type culture collection } \\ \text { HeLa } & \text { American type culture collection } \\ \text { JAR } & \text { American type culture collection } \\ \text { SAOS-2 } & \text { American type culture collection }\end{array}$

Bacteria

BL 21 Escherichia coli

Sterilization department CIC Salamanca

DHa Escherichia coli

Sterilization department CIC Salamanca

Table 6: Technical devices

Technical devices

Apparatus

Applied Biosystems, Hitachi 3100 Genetic Analyzer

Bio Rad Gel Doc 2000

Facs Calibur E 1738

Leica SP5 confocal

Shandon Cytospin 3

Tecan Ultra Evolution

Zeiss Axioplan fluorescence microscope
Software for handling and/or data analysis

Edit Seq; MapDraw (DNAstar)

Bio Rad Gel Doc

Cell Quest

Leica LAS AF

$X$-fluor

Open Lab
Use

DNA sequencing

Agarose gel analysis

Flow Cytometry

Confocal images

Sample preparation for microscopy

Plate reader for protein colorimetry assay

GFP-LC3 punctae counting

145 
Table 7: Primers and siRNAs

Oligonucleotides

Primer name

Sequence

Primers for PCR

forward (fw) or reverse (rev)

\begin{tabular}{|c|c|c|}
\hline forward AIR & GGGCCCAAGCTTGCCACCATGGCGGCGCCGAAGGGGAGCCTC & $\begin{array}{l}\text { Clamp, HindlII restriction site, } \\
\text { Kozak sequence (followed by met) }\end{array}$ \\
\hline fw AIR-E36 & GGGCCCACATGTCCGAATTCGAAGCATTTGACTCGGTCTTGGGT & Clamp, Pcil, EcoRI \\
\hline fw AIR-F282 & GGGCCCACGCGTTTTATGAATGAACAAAAGCTAAACAGAT & Clamp, Mlul restriction site \\
\hline fw AIR-R290 & GGGCCCACGCGTAGATATCCAGCTTCTTCTCTTGTGGTTG & Clamp, Mlul restriction site \\
\hline fw AIR-T262 & GGGCCCGAATTCACGCGTACAGCTGTGGAGCAGTATGTTCCC & Clamp, Mlul restriction site \\
\hline fw AIR-Y268 & GGGCCCGAATTCACGCGTTATGTTCССTCTGAGAAGCTGAGT & Clamp, Mlul restriction site \\
\hline rev AIR-Notl & CCCGGGGCGGCCGCTTTAAATTTCAGAATGAGCAAGATTCAC & Clamp, Notl restriction site, stop codon \\
\hline rev AIR-Pcil & CCCGGGGCACATGTTAATTTCAGAATGAGCAAGATTCAC & Clamp, Pcil restriction site \\
\hline rev AIR- $\Delta 263$ & CCCGGGGCGGCCGCTTTAAGCAACAGTTGCACAACAAATCCAA & Clamp, Notl restriction site, stop codon \\
\hline rev AIR- $\Delta 268$ & CCCGGGGCGGCCGCTTTACTGCTCCACAGCTGTAGCAACAGT & Clamp, Notl restriction site, stop codon \\
\hline rev $A I R-\Delta 274$ & CCCGGGGCGGCCGCTTTACTTCTCAGAGGGAACATACTGCTC & Clamp, Notl restriction site, stop codon \\
\hline rev AIR- $\Delta 282$ & CCCGGGGCGGCCGCTTTACTCCAAGTCACCATAGATACTCAG & Clamp, Notl restriction site, stop codon \\
\hline rev AIR- $\Delta 290$ & CCCGGGGCGGCCGCTTTAGTTTAGCTTTTGTTCATTCATAAA & Clamp, Notl restriction site, stop codon \\
\hline rev AIR- $\Delta 299$ & CCCGGGGCGGCCGCTTTAAACCACAAGAGAAGAAGCTGGATA & Clamp, Notl restriction site, stop codon \\
\hline rev AIR- $\triangle 307$ & CCCGGGGCGGCCGCTTTAATGATCTTCAGTTTTAGATCTAAC & Clamp, Notl restriction site, stop codon \\
\hline rev AIR- $\Delta 317$ & CCCGGGGCGGCCGCTTTACACTTTTGTAGGTAGAGGCCCTGC & Clamp, Notl restriction site, stop codon \\
\hline rev AIR- $\Delta 319$ & CCCGGGGCGGCCGCTTTAAAGATTCACTTTTGTAGGTAGAGG & Clamp, Notl restriction site, stop codon \\
\hline fw LC3A & GGGCCCACATGTCCATGCССTCAGACCGGCCTTTCAAG & Clamp, Pcil restriction site \\
\hline
\end{tabular}

Properties

Clamp, HindIII restriction site,

sequence (followed by met)

Clamp, Mlul restriction site

Clamp, Pcil restriction site

Clamp, Notl restriction site, stop codon

Clamp, Notl restriction site, stop codon

Notl restriction site, stop codon

Clamp, Notl restriction site, stop codon 
MATERIALS-TABLES

\begin{tabular}{|c|c|c|}
\hline rev LC3A & CCCGGGGCGGCCGCTTTAGAAGCCGAAGGTTTCCTGGGAGGC & Clamp, Notl restriction site, stop codon \\
\hline fw LC3B & GGGCCCACATGTCCATGCCGTCGGAGAAGACCTTCAAG & Clamp, Pcil restriction site \\
\hline rev LC3B & CCCGGGGCGGCCGCTTTACACTGACAATTTCATCCCGAACGTC & Clamp, Notl restriction site, stop codon \\
\hline \multicolumn{3}{|c|}{ Primers for protein tagging } \\
\hline HA top & AGCTTGCCACCATGGGCTACCCCTACGACGTGCCCGACTACGCCAGCGGCGGCAGCCA & $\begin{array}{l}\text { HindIII restriction site overhang } \\
\text { Kozak sequence (followed by met) }\end{array}$ \\
\hline HA bottom & CATGTGGCTGCCGCCGCTGGCGTAGTCGGGCACGTCGTAGGGGTAGCCCATGGTGGCA & Pcil restriction site overhang \\
\hline \multicolumn{3}{|c|}{ Primers for sequencing } \\
\hline P8 forward & AGTTAGGCCAGCTTGGCACTTGATGT & \\
\hline P8 reverse & CAGACCTGAGGAAGAGATGG & \\
\hline CD 16 forward & ACCCGCAGACAGCCTCTGCCCTCCC & \\
\hline \multicolumn{3}{|c|}{ ON-TARGETPLUS SMARTPOOL SIRNA } \\
\hline \multirow[t]{4}{*}{ BECN1 } & GAUACCGACUUGUUCCUUA & \\
\hline & GGAACUCACAGCUCCAUUA & \\
\hline & CUAAGGAGCUGCCGUUAUA & \\
\hline & GAGAGGAGCCAUUUAUUGA & \\
\hline \multirow[t]{4}{*}{ ATG5 } & GGCAUUAUCCAAUUGGUUU & \\
\hline & GCAGAACCAUACUAUUUGC & \\
\hline & UGACAGAUUUGACCAGUUU & \\
\hline & ACAAAGAUGUGCUUCGAGA & \\
\hline \multirow[t]{4}{*}{ ATG7 } & CCAACACACUCGAGUCUUU & \\
\hline & GAUCUAAAUCUCAAACUGA & \\
\hline & GCCCACAGAUGGAGUAGCA & \\
\hline & GCCAGAGGAUUCAACAUGA & \\
\hline
\end{tabular}


MATERIALS-TABLES

AIR

GAGUAUCUAUGGUGACUUG

UCACAAGCGCACAGGAAUU

GGAAUUGACUUAAAUCGAA

GAAGAGGAGYYGUACGCAU

Control

NON-TARGETING POOL, CATALOG NUMBER: D-001810-10 
MATERIALS-TABLES

pEAK8-inserts
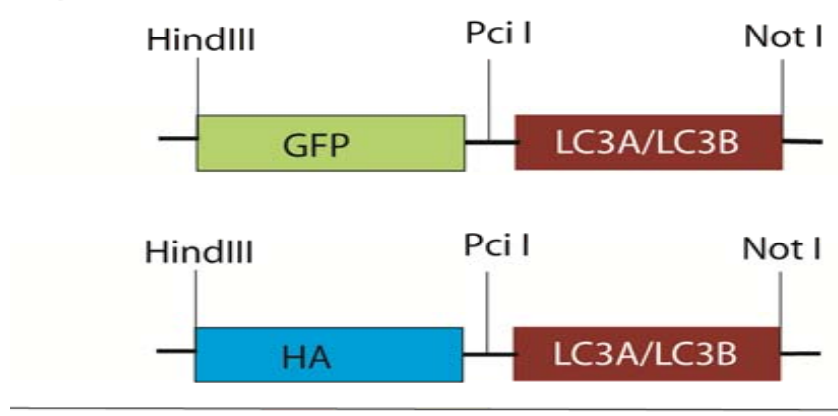

pEAK12-inserts

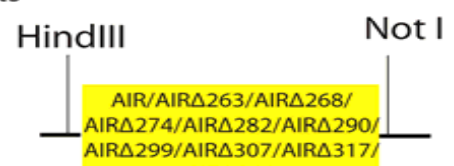
AlR 319
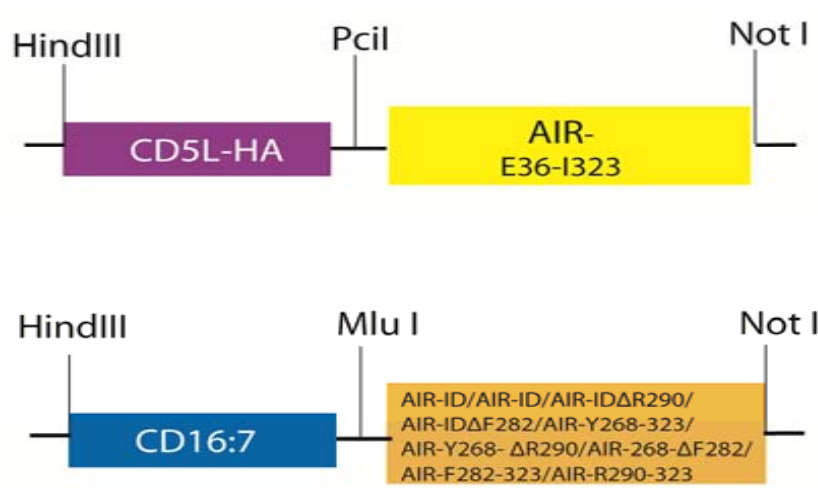

\section{pGEX-4T-AIR-E36-G239}

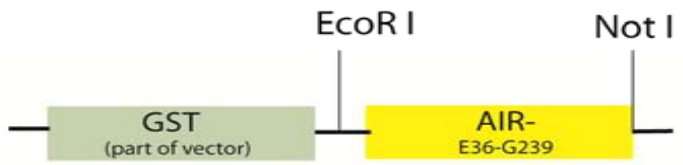

pEAK12-MMP-inserts

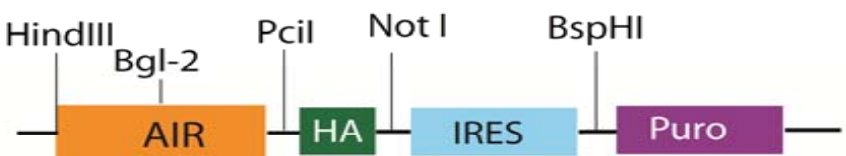

Figure 25: Vectors and inserts used 



\section{Spanish summary}

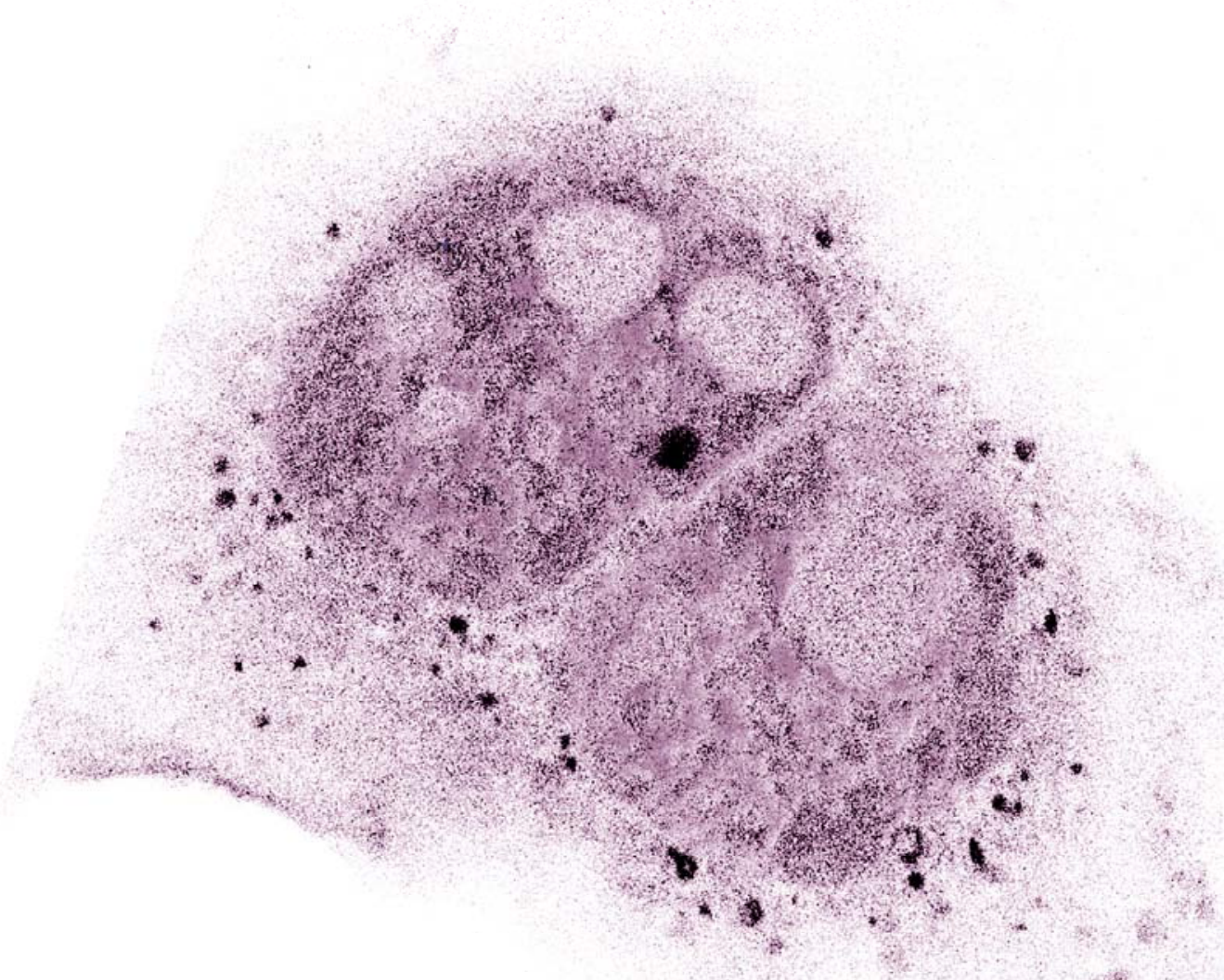





\section{Introducción}

\subsection{Autofagia}

En la célula hay dos vías principales de degradación, el sistema del proteasoma y la degradación en lisosomas. Mientras el proteasoma se encarga de la proteolisis de proteínas dañadas o mal plegadas (Goldberg 2003), el lisosoma está implicado en la eliminación de moléculas de vida media larga. Un proceso especial de degradación en el lisosoma es la autofagia, que consiste en el secuestro de contenido citoplasmático y su transporte al lisosoma, donde se degradan las proteínas y otras moléculas para el subsiguiente reciclaje. La palabra autofagia, que proviene del griego auto - uno mismo - y phagos - comer, y significa autodigestión. Describe un mecanismo de degradación y reciclaje de proteínas especialmente en condiciones de acceso limitado a nutrientes. Como tal, está conservada de levaduras a humanos. Sin embargo, la autofagia ha adquirido funciones en eucariontes superiores que van más allá del mecanismo de respuesta al ayuno, y que incluyen papeles relevantes en el ámbito de la inmunología, la neurología y el cáncer, además de la respuesta celular a estímulos nocivos (Mizushima et al. 2008).

Hasta ahora se han descubierto tres vías de autofagia, la macroautofagia, la microautofagia y la autofagia mediada por proteínas chaperonas. Todas estas formas de autofagia tienen en común que la degradación de las proteínas se realiza en los lisosomas.

La macroautofagia es el tipo de autofagia mejor estudiado, de hecho, en muchas ocasiones se refiere a la macroautofagia simplemente como autofagia. También es el tipo de autofagia en que se centra este trabajo. No obstante, es importante destacar que hay otros tipos de autofagia, dado que la microautofagia y la autofagia mediada por proteínas chaperonas comparten funciones o vías de señalización y proteínas señalizadoras de la maquinaria de la macroautofagia.

\subsubsection{Macroautofagia}

En el caso de la macroautofagia, el contendido citoplasmático que va a ser degradado se envuelve en un orgánulo llamado autofagosoma que está delimitado por una doble o múltiple membrana, una característica típica de esta forma de 
autofagia. Cuando este autofagosoma se fusiona con un lisosoma, el orgánulo formado se denomina autolisosoma y es el lugar de degradación de las proteínas capturadas.

Aunque no se sabe de dónde proviene la membrana del autofagosoma, se ha descrito que su génesis está mediada por las proteínas denominadas proteínas relacionadas con autofagia, (Atgs). En levaduras se han identificado 33 Atgs, muchas de las cuales tienen un homólogo en eucariontes superiores. Anteriormente de los Atgs en la ruta de señalización de (macro)autofagia se encuentra su principal regulador negativo, la Ser/Thr kinasa TOR (díana de rapamicina del inglés target of rapamycin), una molécula que se activa por factores de crecimiento e insulina. Por tanto, su activación inhibe la autofagia dado que la autofagia es un mecanismo que se activa sobre todo en condiciones catabólicas (Esclatine et al. 2009).

La importancia de la macroautofagia como mecanismo de supervivencia en circunstancias de acceso limitado a nutrientes ha sido demostrada en ratones deficientes en mediadores clave de este proceso. Dichos ratones knockout de genes relacionados con autofagia (por ejemplo $\operatorname{Atg} 5$ y $\operatorname{Atg} 7$ ), mueren en el periodo de ayuno postnatal porque son incapaces de activar la macroautofagia (Kuma et al. 2004).

Un ejemplo del papel de la macroautofagia en tumorigénesis son los ratones mutantes Beclin1/Atg6. La deleción de Beclin1 en un solo alelo es suficiente para aumentar el número de tumores espontáneos en estos ratones Beclin1 ${ }^{+/-}$(Qu et al. 2003).

Además existen evidencias de la relevancia de la macroautofagia en neuroprotección. Mediante modelos murinos knockout para un gen central de la maquinaria autofágica, $\operatorname{Atg} 7$, se demostró que su producto génico es necesario para que las neuronas sean funcionales, dado que los ratones carentes de esta proteína en el sistema nervioso central sufren pérdidas de neuronas $y$, por lo tanto, neurodegeneración (Komatsu et al. 2006).

No obstante, a pesar de sus efectos beneficiosos, una macroautofagia excesiva, que conlleva un exceso de degradación de componentes citosólicos, puede producir muerte celular (Pattingre et al. 2005; Elgendy et al. 2011). 


\subsubsection{Autofagia mediada por proteínas chaperonas (CMA)}

Como la macroautofagia, la autofagia mediada por proteínas chaperonas (CMA) se puede inducir por ayuno y tiene un papel importante en neuroprotección y el sistema inmune (Kon \& Cuervo 2010). Sin embargo, se diferencia de la macroautofagia por ser muy selectiva. Las proteínas sometidas a la degradación se translocan a través de la membrana del lisosoma con la ayuda de la chaperona lisosomal hsc70 (del inglés heat shock cognate protein of $\underline{70} \mathrm{kDa}$ ) y el receptor de membrana LAMP-2A, una variante de receptor lisosomal LAMP-2 (del inglés lysosome-associated membrane protein type $\underline{2}$ ) que proviene de un procesamiento alternativo del mismo ARN mensajero. Ambas proteínas, la chaperona y el receptor lisosomal, son necesarias para la CMA. La selectividad de la CMA se consigue por hsc70 que es capaz de reconocer las proteínas que deben degradarse mediante el motivo KFERQ (Dice et al. 1990).

La CMA parece tener relevancia en la presentación de MHC clase-II en la respuesta inmune adaptativa y autoinmune (Zhou et al. 2005), la degradación de proteínas implicadas en la enfermedad de Parkinson (Cuervo et al. 2004) y como mecanismo de supervivencia en condiciones de acceso limitado a nutrientes. Por otro lado se ha demostrado que la CMA se activa cuando la macroautofagia ha sido inhibida, un hecho que implica una interrelación entre los dos tipos de autofagia (Kaushik et al. 2008).

\subsubsection{Microautofagia}

En la vía menos estudiada de autofagia, la microautofagia, los lisosomas secuestran los componentes citosólicos directamente formando invaginaciones de la membrana lisosomal, produciendo así vesículas que finalmente son degradadas en el interior del lisosoma (Uttenweiler et al. 2005). Este mecanismo también puede servir para una reducción del tamaño de la membrana lisosomal, por ejemplo tras fusiones con otras vesículas (Mijaljica et al. 2007). La microautofagia está regulada por el complejo de TOR y otro complejo de proteínas asociado con la membrana vacuolar (el complejo EGO) y su funcionamiento es un proceso dependiente de proteínas Atg (Dubouloz et al. 2005). Aparte de la microautofagia no selectiva, se conocen varios tipos de microautofagia selectiva, por ejemplo la degradación de porciones minúsculas del núcleo, la PMN (del inglés piecemeal microautophagy of the 
nucleus) (Roberts et al. 2003). Además se ha descrito que la microautofagia contribuye a la eliminación de mitocondrias dañadas, siendo importante para la homeostasis celular (Todde et al. 2009).

\subsection{Mecanismos moleculares de macroautofagia}

Hay varios complejos de proteínas que regulan la autofagia. Dado que la autofagia es un fenómeno bastante conservado durante la evolución, las proteínas implicadas en sus vías de señalización también lo están. En la macroautofagia convencional, un estímulo (por ejemplo la limitación de nutrientes) inhibe el complejo formado por TOR y otras proteínas, lo que conlleva la activación del complejo con Atg1/Ulk1. Este complejo de proteínas activa la génesis del autofagosoma. Otro complejo de proteínas importante para la formación del autofagosoma es el formado por Atg6/Beclin1. Además, existen dos sistemas de modificación proteica similar a la ubiquitinación que llevan a cabo el crecimiento de la membrana pre-autofagosomal hasta la formación del autofagosoma completo (Yang \& Klionsky 2010).

\subsection{1. $m T O R$}

mTOR fue descubierta como diana terapéutica de la rapamicina en mamíferos. La droga rapamicina es un antimicótico y un inmunosupresor que inhibe el crecimiento celular en mamíferos. Su diana es el complejo de proteínas mTORC1, uno de los dos complejos que puede formar mTOR y que está implicado en la inducción de la transcripción y la traducción. El segundo complejo, mTORC2, parece ser insensible a la rapamicina y es importante para la organización de los filamentos de actina. Aunque mTORC2 parece influir sobre la autofagia indirectamente por mecanismos de transcripción, mTORC1 es el complejo mejor estudiado y se cree que el más importante para la autofagia, la cual regula negativamente (Wullschleger et al. 2006). Para un mejor entendimiento de los procesos descritos a continuación, ver la figura 2.

En la ruta de señalización, aguas arriba al complejo mTORC1 se encuentran la fosfatidilinositolkinasa-3 clase1 (PI3K del inglés phosphatidylinositol-3-kinase)

$y$, por debajo AKT, que también es conocida como proteína kinasa B (PKB del inglés protein kinase $\underline{B}$ ). Ambas proteínas activan mTORC1 (y por lo tanto inhiben la autofagia), e inhiben otro complejo por encima de mTORC1, TSC1/2 (del inglés tuberous sclerosis complex). La fosfatasa PTEN puede interferir en la ruta PI3K- 
AKT-TSC1/2 en el paso entre la PI3K y AKT anulando el efecto inhibidor que tiene AKT en TSC1/2. Por lo tanto PTEN activa autofagia, y además está descrito como supresor tumoral ( $\mathrm{Li}$ et al. 1997). Otra proteína implicada en la regulación de mTORC1 por TSC1/2 (y en consecuencia en la autofagia) es la kinasa AMPK, un sensor de los niveles de energía a través del ratio ATP/AMP que se activa cuando caen estos niveles. De este modo AMPK activa el complejo TSC1/2 que, a su vez, inhibe al regulador positivo de mTORC1, Rheb. En el caso contrario (que se inhiba TSC1/2 como ocurre como respuesta a la ruta PI3K-AKT), se anularía el efecto negativo de TSC1/2 hacia Rheb. Así la ruta de señalización provocaría una activación de mTORC1 y como consecuencia de ello, se activaría la síntesis de proteínas. En caso de que se elimine la inhibición de mTORC1, se induciría la autofagia mediante el complejo de la kinasa Ulk1 con FIP200 y Atg13 (Esclatine et al. 2009). Este complejo de proteínas se regula a través de fosforilación por mTORC1. Cuando mTORC1 está activado, las proteínas Ulk1 y su activador Atg13 se mantienen hiperfosforilados e inactivos. Así son incapaces de inducir la formación de un fagóforo como precursor del autofagosoma (Ganley et al. 2009).

\subsubsection{Beclin1/Atg6}

Beclin1 es el homólogo mamífero de Atg6 de levaduras y se ha demostrado que es un supresor tumoral haplosuficiente en ratones ( $Q u$ et al. 2003). Beclin1 fue descubierta por su interacción con Bcl-2, miembro de una familia de proteínas reguladoras de apoptosis. La molécula anti-apoptótica $\mathrm{Bcl}-2$ inhibe Beclin1 porque ésta tiene que estar disociada para poder formar el complejo que promueve la nucleación del fagóforo (Liang et al. 1998; Kihara et al. 2001). A partir del fagóforo se desarrolla el autofagosoma de doblemembrana que captura el contenido citosólico. Como la nucleación del fagóforo se ve dinamizada por la fosforilación de fosfatidilinositol, la PI3K-clase-3 Vps34 y su regulador Vps15 son imprescindibles para formar el complejo de proteínas formado en torno a Beclin1. Las proteínas Ambra1, UVRAG y Bif-1 favorecen la formación de dicho complejo, y las dos últimas moléculas mencionados también han sido descritas como posibles supresores tumorales (Takahashi et al. 2007). 


\subsubsection{LC3/Atg8 y dos sistemas de conjugación similar a la ubiquitina.}

Una vez iniciada la génesis del autofagosoma, su crecimiento se lleva a cabo mediante la adición lipídica, y posterior asociación con la membrana autofagosomal, de otra proteína clave en autofagia: Atg8 en levaduras o su homólogo LC3 en mamíferos. En mamíferos, hay varias isoformas del LC3 (LC3A, LC3B y LC3C). Todas estas isoformas están implicadas en el alargamiento de la membrana autofagosomal y sufren una adición lipídica de fosfatidiletanolamina (PE del inglés phophatidylethanolamine) que promueve su asociación con la membrana del autofagosoma (Weidberg et al. 2010). Este proceso depende de dos sistemas semejantes al proceso de conjugación de la ubiquitina en los que participan muchas de las proteínas claves en autofagia.

Recién traducida, LC3 es procesada por la autofagina, (el homólogo de ATG4 de levaduras) produciéndose la forma citosólica, LC3I, que una vez lipidada y unida a la membrana del autofagosoma se denomina LC3II. En los dos sistemas similares al proceso de conjugación con ubiquitina, LC3 juega el papel de modificador análogo a la ubiquitina. Otro modificador similar a la ubiquitina en este sistema es Atg12, mientras que Atg7 funciona como enzima activadora de tipo E1 (E1-like) para el enlace de Atg12 con Atg5 y el enlace de LC3 con PE. La enzima conjugadora E2like para la subsiguiente unión de Atg12-Atg5 con Atg16 es Atg10, mientras la proteína E2-like correspondiente para la interacción de LC3-PE es Atg3. El complejo formado entre Atg12-Atg5 y Atg16L (el homólogo mamífero ATG16 similar de ATG16 de levaduras del inglés ATG16-like) lleva a cabo el enlace covalente entre LC3 y PE (Cecconi \& Levine 2008), funcionando probablemente como sistema E3-like, dado que este proceso de adición lipídica se produce posteriormente a la conjugación de Atg5 con Atg12.

El hecho de que LC3 se modifique en dicho proceso se puede aprovechar para medir autofagia, puesto que hay una correlación directa entre el número de autofagosomas formados y la cantidad de LC3 lipidado (Kabeya et al. 2000). Otra proteína central de este proceso, Atg16L, ha sido relacionada con la enfermedad autoinmune de Crohn, puesto que existen polimorfismos del gen correspondiente en pacientes afectados de esta enfermedad (Hampe et al. 2007). Aunque la formación del autofagosoma se considera clave en la macroautofagia convencional, se han 
descrito tipos de macroautofagia que no dependen de estos sistemas de modificación similares a la ubiquitina. Por ejemplo, en un tipo de macroautofagia independiente de $\operatorname{Atg} 5$ y $\operatorname{Atg} 7$ se forman vesículas de doble o múltiple membrana sin que exista adición lipídica a LC3. No obstante, este proceso dependía de otra proteína clave de macroautofagia, Beclin1 (Nishida et al. 2009).

Sin embargo, Beclin1 tampoco parece imprescindible para la macroautofagia, puesto que se ha demostrado la generación, independiente de Beclin1, de autofagosomas en respuesta al polifenol resveratrol o a inhibidores de Bcl-2 (Scarlatti et al. 2008; Tian et al. 2010).

\subsection{Fusión de autofagosomas con lisosomas}

Una vez formado, el autofagosoma tiene que fusionarse con un lisosoma para que se pueda degradar el contenido del autolisosoma. Antes de la fusión final, los autofagosomas se pueden fusionar con vesículas de los compartimentos endosomales. Parece que esta fusión depende de proteínas en las membranas endosomales, y estos híbridos autofagosoma-endosoma se denominan vesiculas autofágicas tardías (late avs del inglés late autophagic vesicles). Para unirse con un lisosoma, el autofagosoma necesita moverse por los microtúbulos hacia el lugar de la célula donde se encuentra con el lisosoma. Conforme un autofagosoma madura, aumenta la densidad en la membrana de la GTPasa pequeña Rab7, un miembro de la familia de los rabs que regulan muchos procesos de tráfico de membranas. Se ha demostrado que ésto es importante para la fusión completa de un autofagosoma con el lisosoma (Gutierrez et al. 2004b). La vesícula formada tras este evento de fusión se denomina autolisosoma, y en ella se degrada tanto el contenido del autofagosoma como su membrana interior. Tras este proceso las moléculas obtenidas de la degradación de proteínas y orgánulos son devueltas al citoplasma donde se pueden reusar.

\subsection{Autofagia en salud y enfermedad}

Tal y como se ha mencionado más arriba, la autofagia es importante para diversos aspectos tanto fisiológicos como patológicos.

En mamíferos, parece que la autofagia es beneficiosa para prevenir la neurodegeneración. Existen diferentes enfermedades neurodegenerativas que manifiestan defectos en la degradación autofágica de proteínas, lo cual avala esta 
hipótesis. El ejemplo más claro se encuentra en pacientes que padecen la enfermedad de Huntington (HD). Si no se elimina la proteína huntingtina (htt) por autofagia, se forman oligomeros tóxicos en las neuronas de los enfermos (Davies et al. 1997; Ravikumar et al. 2002). Además existen evidencias de que la degradación de la proteína neurotóxica alfa-sinucleína por CMA en la enfermedad de Parkinson (PD del inglés $\underline{P}$ arkinson's disease), o la de otra proteína neurotóxica, el $A \beta$ (beta-amiloide) por macroautofagia en la enfermedad de Alzheimer (AD del inglés Alzheimer's disease) está bloqueada en pacientes que sufren estas patologías (Cuervo et al. 2004; Yu et al. 2005; Boland et al. 2010).

Por otro lado, el hecho de que tanto la $A D$ como la PD sean enfermedades asociadas a la edad apoya la hipótesis de que la autofagia juega un papel importante en el envejecimiento. Así, se sabe que la actividad autofágica decae a lo largo de la vida, y en gusanos se ha demostrado que una activación de la autofagia prolonga la esperanza de vida, mientras que para un gusano mutante con autofagia defectuosa la esperanza de vida se acorta (Toth et al. 2008). Otra enfermedad cuyo riesgo incrementa con la edad es el cáncer, y muchas proteínas de las rutas de señalización de autofagia parecen ser supresores tumorales, como por ejemplo Beclin1 y los reguladores positivos del complejo de proteínas que forma mTORC1 (ver más arriba).

\subsection{Autofagia y el sistema inmune}

Durante los últimos años se ha revelado un papel importante de la autofagia en el sistema inmune. Aunque la mayoría de los procesos descritos están relacionados con la respuesta inmune innata, también hay estudios sobre la participación de autofagia en la presentación de antígenos por el MHC-clase II. Ejemplos de autofagia e inmunología adaptativa son la presentación de productos de patógenos degradados por autofagia a células $\mathrm{T} \mathrm{CD}^{+}$mediante MHC-clase II (Gannage \& Munz 2009). Asimismo la presentación mediante MHC-clase 2 de componentes propios obtenidos por autofagia a células $\mathrm{T} \mathrm{CD}^{+}$durante su desarrollo evita que estas células T desencadenen procesos autoinmunes (Nedjic et al. 2008).

En cuanto a la respuesta inmune innata, la autofagia puede destruir patógenos como el Streptococcus grupo A en estructuras muy parecidas a los autofagosomas convencionales (Nakagawa et al. 2004), o ayudar a la célula a degradar microorganismos por fagocitosis como en el caso de Mycobacterium tubercolosis, 
una bacteria que puede sobrevivir de forma latente en fagosomas humanos mediante un tipo de parasitismo en macrófagos (Gutierrez et al. 2004). Además se ha comprobado que la autofagia está regulada por componentes del sistema inmune innato, dado que citocinas inmunomoduladoras, como el interferón $\gamma$, la inducen. Además, una clase particular de receptores implicados en la regulación de la respuesta inmune, los receptores PRR (receptores de reconocimiento de patrones, es decir patrones de patógenos, del inglés pattern recognition receptor) también pueden desencadenar autofagia (Deretic 2009).

\subsubsection{Xenofagia}

El proceso de degradación de patógenos ha sido denominado xenofagia para hacer hincapié en el hecho de que no se produce degradación de partes de la propia célula sino la eliminación de componentes exógenos. En los estudios más recientes de este fenómeno se ha visto que es un proceso de autofagia selectiva, dado que antes de ser degradadas, las bacterias pasan por un proceso que incluye ubiquitinación e involucra una proteína capaz de unirse a poliubiquitinas, la p62/SQSTM1. Se sabe que esta proteína se degrada por autofagia y que actúa como adaptador para marcar la bacteria para ser autofagocitada, por lo que el marcaje del patógeno con ubiquitina facilita su reconocimiento por esta proteína para promover su degradación (Zheng et al. 2009). Hay varios ejemplos de bacterias que se escapan de este reconocimiento: Salmonella se esconde en vacuolas intracelulares (Birmingham et al. 2006), Listeria monocytogenes se enmascara con otros componentes celulares y así parece de la célula hospedadora (Yoshikawa et al. 2009) y Shigella flexneri secreta IscB, una proteína que inhibe la interacción de la maquinaria autofágica con otra proteína bacteriana, virG, a través de la cual la bacteria sería reconocida y sometida a eliminación autofágica (Ogawa et al. 2005). Aparte de bacterias, también hay virus que son combatidos mediante autofagia, como por ejemplo el virus del herpes simplex tipo 1 (HSV-1). Este virus también ha desarrollado un mecanismo de defensa contra la maquinaria autofágica de la célula, que se basa en la expresión de la proteína ICP 34.5 la cual se une e inhibe a Beclin1 (Orvedahl et al. 2007). 


\subsubsection{Receptores de reconocimiento de patrones-PRR}

Como se ha mencionado más arriba, los PRR responden a moléculas de organismos exógenos, por ejemplo ácidos nucleicos virales o componentes de la membrana bacteriana. Estos ligandos han sido denominados PAMPs (patrones asociados con patógenos del inglés pathogen associated molecular patterns) (Delbridge \& O'Riordan 2007; Deretic 2009). Existen cuatro subgrupos de los PRR. Dos de ellos, los TLR (del inglés toll like receptor) y los NLR (del inglés nod like receptor), parecen especialmente interesantes con relación con la autofagia. Muchos miembros de la familia NLR están implicados en la formación de los inflamosomas, que llevan a cabo la maduración de las citocinas proinflamatorias una vez que se ha detectado al intruso microbiológico (Martinon et al. 2007). Otros miembros importantes de la familia de los NLR son Nod1 y Nod2. Ambos pueden inducir autofagia tras la estimulación con PGN (peptidoglicano de bacterias), un componente de la membrana bacteriana. Aunque aún se desconoce cómo se desencadena dicho efecto (dado que no se activan las típicas proteínas señalizadores implicadas en la ruta convencional de los Nods), se ha visto que estas moléculas dirigen Atg16L a la zona de la membrana celular donde entra la bacteria y promueven ahí la generación de autofagosomas (Travassos et al. 2010).

\subsubsection{Receptores de tipo toll-TLR}

Los TLR se encuentran principalmente en la membrana plasmática, y al ser proteínas transmembrana pueden interaccionar con los PAMP en regiones que topológicamente están fuera de la célula. Juegan un papel importante en la fagocitosis de patógenos (Blander \& Medzhitov 2004), y su activación por PAMP virales o bacterianos conlleva la producción de citocinas e interferones por rutas de señalización que incluyen moléculas adaptadoras como MyD88 o TRIF (Saitoh \& Akira 2010).

Aparte de facilitar la presentación de antígenos a los TLR (Deretic 2009), la autofagia está implicada también en la respuesta a la estimulación de estos receptores. Existen varios estudios que relacionan la autofagia inducida por patógenos con los TLR. Por ejemplo, un estudio en macrófagos demuestra que la estimulación de TLR4 por LPS (lipopolisácaridos de bacterias) mejora la eliminación del microorganismo intracelular M. tubercolosis a través de la inducción de autofagia 
(Xu et al. 2007). Además, la eliminación por autofagia de patógenos que residen dentro de la célula también se puede ver favorecido por tratamiento con PAMPs virales, como es el caso de los SsRNA (del inglés single stranded ribonucleid acid) que activan TLR7 (Delgado et al. 2008).

Los macrófagos son células especializadas en la fagocitosis de patógenos, un proceso en el que participan los TLR y que acaba con la fusión del fagosoma con un lisosoma y la subsiguiente degradación del microbio. Como parece ocurrir en el caso de M. tuberculosis (Gutierrez et al. 2004), la autofagia podría ser un mecanismo que mejora estas propiedades fagocíticas para favorecer la eliminación de bacterias, y de hecho, la activación por TLR conecta directamente la fagocitosis con la autofagia. Así, se ha demostrado que la activación de TLR en macrófagos dirige el marcador autofágico LC3 hacia los fagosomas (Sanjuan et al. 2007). Al contrario que en el caso de autofagosomas convencionales, estas vesículas sólo tienen una membrana y aún se desconoce hasta qué punto estos fenómenos son similares a la autofagia convencional aunque sí se conoce su dependencia de moléculas que constituyen la maquinaria autofágica basal.

\subsection{Autofagia y el cáncer}

Por lo que se conoce hasta el momento, la autofagia parece ser beneficiosa para la prevención del cáncer. Varias proteínas pro-autofágicas como Beclin1 o URAG son supresores tumorales, mientras que inhibidores de autofagia como Akt, $\mathrm{Bcl}-2$ o la PI3K-clase1 son conocidos oncogenes (Morselli et al. 2009). Existen varias hipótesis para explicar este nexo, entre ellas que la inhibición de autofagia favorece la necrosis y la inflamación, siendo ambos procesos implicados en la inducción de tumorigenesis (Degenhardt et al. 2006). Por otro lado, la autofagia degrada orgánulos defectuosos que si no son eliminados podrían provocar daños a la célula (Lemasters 2005).

Sin embargo, mientras que en la génesis del tumor la autofagia parece restringir la transformación, en la célula tumoral promueve podría promover la supervivencia celular en circunstancias de acceso limitado a nutrientes, o en respuesta a medicamentos anti-tumorales (Vazquez-Martin et al. 2009). En este contexto es interesante mencionar que el supresor tumoral más mutado en tumores, p53 (Vogelstein et al. 2000), induce o suprime autofagia según las circunstancias. Como factor de transcripción nuclear induce autofagia, por ejemplo mediante la 
inducción de la proteína pro-autofágica DRAM (modulador de autofagia regulada por deterioro, del inglés damage related autophagy modulator) (Crighton et al. 2006). No obstante, p53 citosólico también inhibe autofagia, tal y como se ha descrito al eliminar esta proteína específicamente del citosol en experimentos con células tumorales (Tasdemir et al. 2008).

\subsection{Autofagia como mecanismo de muerte celular programada}

Otro aspecto interesante en cuanto a la función de la autofagia en cáncer es su capacidad de inducir muerte celular programada. Ya que la maquinaria de apoptosis es defectuosa en muchos cánceres (Yonish-Rouach et al. 1991), la autofagia podría funcionar como una ruta alternativa para matar a la célula maligna. De hecho, se ha demostrado que la autofagia puede funcionar como una vía de muerte celular cuando la apoptosis es defectuosa, como es el caso en muchos tumores (Kanzawa et al. 2005). Esta vía de muerte celular no depende de caspasas como es el caso de la apoptosis, sino de proteínas Atg y transcurre con cambios morfológicos y bioquímicos diferentes a la apoptosis (Shimizu et al. 2004).

\subsubsection{Apoptosis}

Hay dos maneras de iniciar apoptosis. Una de ellas es la vía intrínseca, en la que la mitocondria libera proteínas pro-apoptóticas tras la permeabilización de su membrana externa. Esta permeabilización está regulada por la familia de proteínas $\mathrm{Bcl}-2$, que se puede dividir en tres subgrupos: las proteínas anti-apoptóticas (p. ej. $\mathrm{Bcl}-2$ o $\mathrm{Bcl}-\mathrm{xl}$ ), las proteínas pro-apoptóticas (p. ej. Bak y Bax), y las proteínas que se unen e inhiben los Bcl-2 anti-apoptóticas (p. ej. Bim o Puma) y que de ese modo liberan a las moléculas proapoptóticas Bak y Bax de su inhibición, y favorecen así el proceso apoptótico. Este último subgrupo se denomina proteínas $\mathrm{BH} 3-$ only, haciendo referencia a que sus miembros sólo presentan un dominio de $\mathrm{BH}$ (homología de $\mathrm{Bcl}-2$ del inglés $\underline{B} \mathrm{cl}-2$ homology) mientras las otras $\mathrm{Bcl}-2$ proteínas tienen varios dominios $\mathrm{BH}$.

La otra vía de inducción de apoptosis es la vía extrínseca que se activa por las proteínas de la familia TNF (factores de necrosis tumoral, del inglés tumour necrosis factor) y sus receptores. Ambas vías culminan en la activación de caspasas que desencadenan el programa de muerte celular de forma irreversible (Martin \& Green 1995). Morfológicamente, este proceso incluye el encogimiento de la célula y su 
núcleo, y la degradación de compartimentos subcelulares, mientras que se pueden observar alteraciones morfológicas típicas en la membrana celular (blebbing o rugosidades de la membrana). Al final, la cromatina se condensa, y el ADN se fragmenta en la característica escalera de ADN que se puede visualizar en geles de agarosa (Cohen et al. 1994). Los restos de la célula se eliminan por fagocitosis llevado a cabo por las células vecinas o macrófagos (Savill \& Fadok 2000). Durante bastante tiempo se creía que la apoptosis era la única vía de muerte celular programada, y que si se podía ejecutar la célula moría por necrosis, que parecía accidental y no regulada genéticamente (Kerr et al. 1972). Pero en los últimos años se ha visto que existen otros tipos de muerte celular programada, como la muerte celular autofágica o la necrosis programada (Edinger \& Thompson 2004; Hotchkiss et al. 2009).

\subsubsection{Muerte autofágica}

En cuanto a la muerte autofágica hay ejemplos de inhibición de muerte celular por inactivación de proteínas atg in vivo en C. elelgans y Drosophila (Berry \& Baehrecke 2007; Samara et al. 2008). Además hay varios ejemplos in vitro de este mismo fenómeno, como la muerte inducida por la proteína $\mathrm{BH}$-only, BNIP3, que se ve acompañada por la activación del marcador autofágico LC3 (Kanzawa et al. 2005), o RAS oncogénico, que provoca muerte celular autofágica inhibible por silenciamiento de genes centrales en autofagia como Atg5 y Beclin1 (Elgendy et al. 2011).

No obstante, hay desacuerdo en el mundo científico sobre si la muerte autofágica existe fisiológicamente, o si es un fenómeno que sólo ocurre in vitro o en circunstancias de inhibición de apoptosis. Estas dudas surgen con motivo de que en la mayoría de los casos la autofagia es una estrategia de supervivencia celular (Kroemer \& Levine 2008). Aún así parece haber una interrelación muy relevante entre autofagia y apoptosis. Por ejemplo, varias proteínas de las vías de señalización de autofagia y de apoptosis interaccionan unas con otras. Uno de los ejemplos más notables de este fenómeno es la interacción existente entre la proteína pro-autofágica Beclin1 y su inhibidor, la proteína anti-apoptótica Bcl-2 (Pattingre et al. 2005). 


\subsection{Monitorizar autofagia en investigación}

El marcador de autofagia más usado es el LC3. Mientras la mayoría de las proteínas Atg sólo están presentes en el autofagosoma de manera transitoria, el LC3 permanece en la membrana hasta su degradación en el autolisosoma (Yang \& Klionsky 2010). Dado que sufre una adición lipídica cuando se induce autofagia y la formación de autofagosomas, LC3 es una buena herramienta para monitorizar el proceso. Para visualizar la adición lipídica de LC3 se usan las técnicas de Western blot o microscopía de fluorescencia (Kabeya et al. 2000). En los geles de Western blot la forma del LC3 lipidada y unida a la membrana del autofagosoma (LC3II), migra más rápido que LC3I citosólico. Para microscopía de fluorescencia se pueden usar formas marcadas con GFP (GFP-LC3). En condiciones normales LC3 tiene una distribución citosólica que en el microscopio se ve como difusa. En cuanto se induce autofagia, LC3 se trasloca a la membrana autofagosomal y adquiere una distribución vesicular.

La herramienta más adecuada para visualizar la característica membrana doble de los autofagosomas es mediante microscopía electrónica, ya que esta técnica presenta una elevada resolución (Arstila \& Trump 1968; Yla-Anttila et al. 2009).

Desafortunadamente no hay más marcadores fiables para monitorizar el proceso autofágico. Debido a que en mamíferos el número de lisosomas no correlaciona bien con el número de autolisosomas, los agentes lisosomotrópicos como el naranja de acridina no son buenos marcadores (Mizushima et al. 2004). Posiblemente, la degradación de la proteína p62/SQSTM1 documenta la actividad autofágica puesto que se degrada por autofagia. Sin embargo, no se sabe con exactitud si p62 se degrada exclusivamente por autofagia (Zheng et al. 2009).

\subsubsection{Inhibición de autofagia}

Para inhibir autofagia se debe considerar preferentemente el silenciamiento o knockout de genes antes de la inhibición por fármacos. Sin embargo, es necesario tener en cuenta que muchas proteínas Atg tienen isoformas que podrían ser funcionalmente redundantes, por lo que un silenciamiento de un único gen podría no ser suficiente para generar un fenotipo acusado. No obstante, los knockdown de Atg5, Atg7 o Beclin1 muestran una buena inhibición de autofagia en muchos sistemas, y son ampliamente utilizados en este contexto (Mizushima et al. 2010). 
Existen varios tipos de fármacos inhibidores de autofagia que se suelen utilizar en investigación, y que inhiben la autofagia en diversos pasos. Sin embargo, ninguno de ellos es específico. Los inhibidores de la PI3K wortmanina (WM) o 3metiladenina (3-MA) ejercen su actividad en el complejo de proteínas que incluye Beclin1 y la PI3K-clase3 (Vps34), inhibiendo la formación del autofagosoma. Sin embargo, este complejo de proteínas está por debajo de mTOR y éste a su vez se regula por una PI3K-clase1, lo que implica un efecto opuesto al ejercido por la PI3K-clase3, puesto que la PI3K-clase 1 inhibe autofagia y la PI3K-clase 3 la induce. Por eso es posible que los inhibidores de la PI3K presenten un efecto inhibidor variable en diferentes sistemas de autofagia (Mizushima et al. 2010).

Más adelante en el proceso de autofagia, se puede inhibir la degradación en los autolisosomas mediante un tratamiento con inhibidores de proteasas lisosomales, como E64d y pepstatina. Un inhibidor de la acidificación lisosomal, Bafilomicina A1, también se puede usar con esta finalidad (Klionsky et al. 2008).

\subsection{Screening de alto rendimiento para identificar inductores de muerte celular}

El proyecto de esta tesis ha sido el estudio de una nueva proteína de membrana que presenta la capacidad de inducir autofagia. Esta nueva molécula proviene de un screening funcional abordado para identificar moléculas inductoras de muerte celular al ser sobreexpresadas. El screening se realizó a partir de una genoteca de ADNc (ADN complementario) obtenido de células $T$ humanas activadas por interleuquina2 y clonado en el vector pEAK. EI ADN de esta librería se cotransfectó con GFP en células HEK-293T. La genoteca se dividió previamente en grupos de clones con un tamaño suficientemente pequeño como para poder detectar la actividad de un único clon inductor de muerte celular. La muerte se detectó por microscopía de fluorescencia a través de los cambios morfológicos sufridos por las células transfectadas. La progresiva subdivisión de los grupos positivos permitió la obtención de un clon aislado que retiene la actividad inductora de muerte celular.

En el screening se distinguieron tres diferentes morfologías de muerte celular programada. La mayoría de las moléculas identificadas indujeron alteraciones morfológicas típicas de la apoptosis, como el blebbing y el encogimiento celular. Además se identificó un tipo de muerte que provoca que las células se desprendan del sustrato y adopten un aspecto globular. Esta morfología ha sido previamente 
descrita en relación a la muerte celular autofágica (Shimizu et al. 2004). La tercera morfología observada se caracteriza por la aparición de una extensiva vacuolización citoplasmática, un proceso también observado en la muerte celular de tipo paraptótico (Sperandio et al. 2000).

\subsection{El clon 9-1.4.-P15}

El clon 9-1.4.-P15 que codifica para la proteína central de esta tesis induce muerte celular morfológicamente relacionada con muerte autofágica. Estructuralmente, esta proteína parece ser transmembrana y con topología de tipo I (C-terminal intracelular), y está asociada sistemáticamente en las bases de datos como Tmem59 (del ingles transmembrane protein 59). En el momento de su identificación esta molécula era desconocida. Sin embargo, recientemente se ha publicado un trabajo que relaciona a esta proteína con la glicosilación compleja de proteínas en el aparato de Golgi y con la maduración de APP (del inglés amyloid beta precursor protein, proteína precursora de amiloide), un proceso central en la patogénesis de la enfermedad de Alzheimer (Ullrich et al. 2010). 


\section{Resultados y discusión}

\subsection{El clon 9-1.4.-P15 induce muerte autofágica}

Para caracterizar mejor el tipo de muerte celular inducida por el clon 9-1.4.-P15, se investigó si dicho proceso dependía de caspasas. Con este fin, se transfectaron células HEK-293 con el clon o con un control positivo de muerte dependiente de caspasas, la proteína conocida por inducir apoptosis RIP3. A continuación las células fueron tratadas con un inhibidor de caspasas, ZVAD.fmk, con objeto de evaluar su capacidad de inhibir el proceso de muerte celular. Como se esperaba, en el caso de RIP3, ZVAD.fmk inhibió la casi totalidad de la muerte celular, mientras que la muerte inducida por 9.-1.4.-P15 no fue abolida tras inhibir las caspasas (figura 10B). Junto con la morfología globular (figura 10A) que mostraba la célula al morir, esto sugería que la muerte podría ser autofágica, tal y como se ha descrito para fibroblastos embrionarios murinos deficientes en apoptosis y tratados con etopósido. En este caso, la muerte dependía de las proteínas centrales de autofagia Atg5 y Beclin1 y producía un fenotipo también redondeado y globular (Shimizu et al. 2004). Como una de las características más importantes de autofagia es la formación del autofagosoma con su característica doble membrana que sólo se puede visualizar por microscopía electrónica, el siguiente paso fue obtener imágenes de células transfectadas con el clon mediante dicha técnica. Como era de esperar, se encontraron varios orgánulos de este tipo en células HEK-293 transfectadas con 9-1.4.-P15 (figura 10C). En los siguientes ensayos empleamos LC3, que es el marcador más usado para medir autofagia (ver introducción, sección 1.2.3. y 1.7.). Ya que trabajamos con células transfectadas es ventajoso cotransfectar dicho marcador. Además de poder usar versiones marcadas (como GPF-LC3 para microscopía de fluorescencia, o HA-LC3 que se puede detectar por un anticuerpo más sensible que el LC3 endógeno), el hecho de que se cotransfecten el marcador y el clon permite analizar sólo las células transfectadas. De este modo se mide sólamente el efecto causado por la proteína cotransfectada. En la primera ronda de experimentos se emplearon células HEK-293T para tener el mismo tipo de células que en el screening. Se transfectaron con el reportero de autofagia, LC3A, y como control positivo se usó el homólogo de BNIP3, BNIP3L. Esta molécula constituye un control positivo adecuado puesto que se ha visto que la 
sobreexpresión de BNIP3 induce muerte celular autofágica, y que BNIP3L y BNIP3 están implicadas en muerte autofágica inducida por trióxido de arsénico (Kanzawa et al. 2005). En ambos experimentos, el de microscopía de fluorescencia y el de Western blot, el reportero dio positivo para el clon como inductor de autofagia. En las imágenes de microscopía de fluorescencia se ve que la señal difusa del GFPLC3 cambia a una distribución moteada, tal y como se observa al formarse autofagosomas. De acuerdo con esto, en Western blot se ve una banda de LC3II incluso más abundante que en el control positivo, por lo que parece que P15 induce autofagia más fuertemente que BNIP3L (figura $11 \mathrm{~A} / \mathrm{B}$ ).

\subsection{9-1.4-P15 codifica para una proteína transmembrana}

Como se mencionó en la introducción, el nuevo clon codifica para la proteína Tmem59 y su nombre hace referencia a que es una proteína transmembrana. Un nombre alternativo es proteína unida a la membrana hepática (del ingles liver membrane-bound protein) y el gen respectivo se denomina TMEM59 o C1orf8. Según los pronósticos de las bases de datos Smart de Heidelberg y Uniprot del Instituto Suizo de Bioinformática la proteína sin procesar pesa 36,2 kDa y tiene 323 aminoácidos (aa), lo cuales contienen desde el N-terminal, una secuencia leader que la lleva a la membrana, seguida por una parte previsiblemente extracelular, una región transmembrana y el dominio probablemente intracelular en el C-terminal. La región leader parece ser de 35 aa y la región transmembrana se encuentra según Uniprot (www.uniprot.org) entre aa 239-259, y según Smart (http://smart.emblheidelberg.de) entre aa 240-262 (figura 11C). Por su estructura y su capacidad de inducir autofagia la nueva molécula ha sido denominada receptor inductor de autofagia, AIR (del inglés autophagy inducing receptor).

Al analizar el domino intracelular en la base de datos Locate de localización subcelular (http://locate.imb.uq.edu.au/), se encontraron varios motivos implicados en transporte de proteínas, por ejemplo de endocitosis y tráfico endosomal, o de exportación del retículo endoplasmático.

AIR está altamente conservado en mamíferos y en menor medida en peces. Sin embargo, una característica de la autofagia es que muchas de las proteínas implicadas en ella se han conservado en la evolución desde levaduras a humanos, y AIR no tiene un homólogo en levaduras (figura 12). No obstante, la autofagia ha ganado funciones durante la evolución, un ejemplo de lo cual es Atg16. A pesar de 
que Atg16 está implicada en la maquinaria central de autofagia, su homólogo en humanos es mucho más largo que en levaduras, y juega un papel importante en la autofagia relacionada con procesos inmunológicos sugiriendo que el engrandecimiento de la proteína conlleva una ganancia de funciones (Hampe et al. 2007). Debido a este aumento de tareas que la autofagia ha adquirido en eucariontes superiores, no sorprendería que existieran proteínas implicadas en autofagia que no tienen homólogo en levaduras. Así, experimentos preliminares argumentan a favor de una función de AIR en neuroprotección o inmunología y no en autofagia como respuesta a condiciones de acceso limitado a nutrientes, dado que un silenciamiento de AIR en células SAOS-2 apenas afectó a la autofagia inducida por ayuno.

\subsection{AIR es un inductor de autofagia general}

Tras haber identificado AIR en células HEK-293T de riñón embrionario humano que se caracterizan por tener niveles altos de expresión de la proteína transfectada, quisimos testar si AIR funcionaba como inductor de autofagia independientemente del tejido y tipo celular y con niveles menores de proteína. Para eso los ensayos de contransfección con el reportero autofágico LC3 se llevaron a cabo en células HEK293 normales que se diferencian de los HEK-293T en que expresan menos la proteína ectópica, y además usamos dos líneas celulares humanas tumorales que provienen de tejidos diferentes, las células Jar de coriocarcinoma de placenta y las células SAOS-2 de osteosarcoma. Además se empleó una segunda isoforma del reportero autofágico, el LC3B. Como se puede detectar en Western blots y en microscopía de fluorescencia, la cotransfección de AIR con HA-LC3 A/B o GFPLC3 A/B respectivamente, induce la adición lipídica que produce LC3II en todas las líneas celulares y con ambas isoformas del reportero. Al comparar los niveles de LC3II inducido por AIR con las actividades por BNIP3L, se observa que son incluso mayores que en el control positivo. Esta constatación se corrobora mediante contajes de los puntos formados por GPF-LC3 en células que expresan AIR o BNIP3L ectópicamente. Con las dos isoformas de LC3 se cuentan más vesículas en las muestras transfectadas con AIR, aunque, sólo con LC3B esta diferencia es considerable (figura 13).

Por tanto, los datos obtenidos indican que AIR es un inductor general de autofagia, dado que tiene el mismo efecto en todas las líneas celulares estudiadas, incluso 
cuando se expresa a niveles reducidos, como en experimentos con las células SAOS-2 que se caracterizan por niveles bajos de proteína transfectada. Además AIR es capaz de inducir autofagia más fuertemente que la proteína $\mathrm{BH} 3-$ only BNIP3L, un conocido inductor de autofagia.

\subsection{Anticuerpos contra AIR}

Para seguir con la caracterización de esta nueva proteína era importante poder detectarla, pero desafortunadamente no hay anticuerpos comerciales contra AIR. Con el fin de producir uno en el laboratorio, se inmunizaron conejos y pollos con una proteína de fusión de la parte supuestamente extracelular de AIR con GST. A continuación, se purificaron los anticuerpos por cromatografía de afinidad. Aunque estos anticuerpos fueron capaces de detectar AIR sobre-expresado en Western blots en todas las líneas celulares testadas (HEK-293, Jar, SAOS-2, HeLa y HCT116), no fue posible detectar la proteína endógena. No obstante, en células transfectadas los anticuerpos detectan una banda en Western blots a la altura esperada, que está ausente en células no transfectadas (figura 14A).

El problema de la ausencia de sensibilidad del anticuerpo frente a AIR endógeno en ensayos de Western blot se pudo solucionar por inmunoprecipitaciones con el anticuerpo de conejo y la siguiente inmunodetección en Western blots mediante el anticuerpo generado en pollo. De esta manera se pudo detectar la proteína endógena en todas las líneas investigadas (figura 14B). Para comprobar si los anticuerpos eran específicos, se abordaron estudios de silenciamiento de AIR en células HeLa y SAOS-2, ya que en estos tipos celulares se encontró la mayor expresión de AIR endógeno. En células transfectadas con ARNi (ARN de interferencia) contra AIR la banda detectada por el anticuerpo desaparece, mientras que células transfectadas con un ARNi control (que carece de dianas en mamíferos) mantiene la señal original (figura 14C). Además, dado que en estos ensayos las células SAOS-2 mostraron la mayor expresión endógena de AIR, realizamos una inmunofluorescencia en esta línea celular frente a dicha proteína y finalmente fuimos capaces de detectar la proteína endógena. Tras teñir las células permeabilizadas con el anticuerpo de conejo se pudieron ver estructuras punteadas mediante microscopía de fluorescencia que desaparecieron después de un silenciamiento con ARNi contra AIR (figura 14D). 


\subsection{Localización subcelular de AIR}

El hallazgo de una herramienta para visualizar AIR endógeno por inmunofluorescencia permitió estudiar la localización subcelular de la proteína. Debido a su estructura de proteína transmembrana, al hecho de que la parte supuestamente intracelular presenta motivos de endocitosis y a que en unos experimentos preliminares se detectó una forma marcada y sobre-expresada de AIR en la membrana plasmática (figura 11D), la superficie celular fue el primer sitio en donde se evaluó la presencia de AIR. Sin embargo, una tinción de la superficie con el anticuerpo de ratón a $4^{\circ} \mathrm{C}$ y en presencia de azida sin haber permeabilizado la célula anteriormente dio negativo para la presencia de AIR. Entonces intentamos el mismo tipo de tinción en células que habían sido transfectadas con mutantes de dinamina capaces de inhibir algunos procesos de endocitosis. Sin embargo, estos mutantes tampoco pudieron retener la proteína en la superficie, aunque otro inhibidor de endocitosis sí fue capaz. Tratando las células con cloroquina, que inhibe la acidificación de endosomas tardíos, se detectó AIR en la membrana plasmática de células SAOS-2. Este resultado corroboró nuestra hipótesis de que AIR se expresa en la membrana plasmática (figura 16C). No obstante, AIR parece endocitarse muy rápidamente, por lo que la superficie celular no es el sitio prevalente de AIR. En un artículo publicado durante nuestros estudios, los autores proponen el aparato de Golgi como localización subcelular de AIR (Ullrich et al. 2010). Con el proposito de reproducir este resultado teñimos células SAOS-2 simultáneamente para AIR y el marcador del aparato de Golgi GM130. Aunque este es el mismo marcador utilizado por Ullrich et al, no hubo apenas colocalización. Por el contrario, marcadores de los compartimientos lisosomales y endosomales, como el CD63 y LAMP2 (del inglés lysosome associated membrane protein $\underline{2}$ ), mostraron bastante colocalización (figura 15). El hecho de que detectemos AIR en estos compartimientos es consistente con su presencia en la superficie tras inhibir la endocitosis.

\subsection{La posible implicación de endocitosis para la autofagia inducida por AIR}

El tratamiento de las células con cloroquina fue la única manera de prevenir la internalización de AIR. Es interesante mencionar que esta droga inhibe la circulación de otra proteína, LAMP1. Esta proteína viaja desde la superficie celular hacía los 
endosomas y el lisosoma. Desde ahí vuelve a la membrana plasmática para su reciclaje. Este tipo de circulación también se conoce en receptores que interaccionan con su ligando en la superficie de la célula, los cuales son endocitados, llegan al lisosoma, donde desasocian de su ligando y desde donde regresan a la membrana plasmática (Lippincott-Schwartz \& Fambrough 1987). Aparte de la superficie celular, AIR localiza en los compartimentos endosomaleslisosomales $\mathrm{y}$, por tanto, es posible que circule entre la membrana plasmática y los lisosomas como lo hace LAMP100. Otra posibilidad sería que AIR no se recicle al llegar a los lisosomas sino que se degrade.

Se sabe desde hace tiempo que los compartimentos de la vía endosomal pueden fusionarse con autofagosomas (Orsi et al. 2010). Además, recientemente se ha demostrado que las membranas de los autofagosomas pueden originarse en la membrana plásmatica por mecanismos de endocitosis. Se descubrió que una de las proteínas claves en la génesis del autofagosoma, Atg16L, interacciona con la proteína clatrina (presente en las vesículas endocíticas que se forman a partir de invaginaciones de la membrana celular) en un proceso que da lugar a las estructuras precursoras del autofagosoma, los fagóforos (Ravikumar et al. 2010). Esta publicación es especialmente interesante porque la procedencia de los fagóforos no está clara, aunque últimamente varios orgánulos (entre ellos el aparato de Golgi, el retículo endoplasmático y la mitocondria) han sido propuestos como origen de las membranas dobles autofagosomales (Hayashi-Nishino et al. 2009; Hailey et al. 2010; van der Vaart et al. 2010).

Dado que AIR está siendo endocitado constantemente, el hecho de que las membranas autofagosomales pueden formarse por endocitosis sugiere que AIR podría estar implicado en este proceso y formar parte de los fagóforos. Una manera de investigar esta hipótesis sería mediante experimentos de triple tinción de clatrina, AIR y Atg16L o co-inmunocprecipitaciones entre AIR, clatrina y Atgs implicados en la génesis del autofagosoma. Una posible interacción de AIR con el complejo aglutinado por Atg16L (Atg5, Atg12, Atg16L) sería de especial interés puesto que Atg16L parece ser importante en el sistema inmune dado que se han descrito polimorfismos en Atg16L relacionados con la enfermedad autoinmune de Crohn. Además, la translocación de Atg16L hacia la membrana plasmática no sólo fue descrita en la formación de fagofaros en autofagia inducida por ayuno, (Ravikumar et al. 2010) sino también por activación de Nods en un proceso autofágico 
implicado en la degradación de bacterias. En este caso se vio que los Nods dirigen Atg16L a la superficie celular donde los patógenos son envueltos en autofagosomas para ser eliminados (Travassos et al. 2010). Dado que sospechamos que AIR juega un papel en autofagia inducida principalmente por estímulos diferentes al ayuno, y que puede estar involucrado en un proceso importante para eucariontes superiores, una hipotética interacción de AIR con la maquinaria autofágica insinuaría su posible implicación en inmunología innata y degradación de patógenos.

\subsection{Estudios del dominio funcional de AIR}

Según dos bases de datos diferentes, la parte C-terminal de AIR es predeciblemente intracelular. La detección de la proteína en la superficie de células no permeabilizadas con un anticuerpo frente al dominio $\mathrm{N}$-terminal, refuerza la idea que la parte $\mathrm{N}$-terminal está fuera de la célula y la parte $\mathrm{C}$-terminal dentro. Con el objetivo de evaluar si la parte intracelular (aminoácidos 263-323) es necesaria para inducir autofagia, se transfectaron células HEK-293, Jar y SAOS-2 con dos isoformas $(A / B)$ de nuestro reportero de autofagia LC3 y se compararon los niveles de LC3II en los puntos transfectados con AIR-FL (la proteína entera, del inglés full length) y AIR- $\triangle I D$ (la proteína sin dominio intracelular, del inglés intracellular domain). Como se puede ver en los Western blots, la activación del HA-LC3 por AIR- $\triangle I D$ en todas las líneas celulares testadas (HEK-293, Jar y SAOS-2) y con ambos isoformas del reportero bajó a niveles comparables a los de las células transfectadas con el vector vacío, usado como control negativo (figura 17C). Además, las imágenes de microscopía de fluorescencia de las células cotransfectadas con GFP-LC3 (A/B) y el mutante que carece de la parte Cterminal o AIR-FL o el vector vacío muestran un resultado consistente con los Western blots. Mientras que en las muestras transfectadas con la proteína entera AIR-FL, pudimos observar gran cantidad de vesículas, en las transfectadas con AIR- $\triangle I D$ el GFP-LC3 permanece en el citosol (figura 17A). Al cuantificar los puntos verdes por célula en la línea HEK-293 pudimos ver que la eliminación del dominio intracelular disminuye el número de vesículas inducidas hasta niveles similares al vector vacío (figura 17B). Estos resultados confirman que el dominio intracelular es necesario para inducir autofagia.

Teniendo en cuenta que la parte intracelular es la parte efectora, quisimos averiguar la secuencia mínima de este dominio con capacidad de inducir autofagia. Con ese 
objetivo se construyeron los siguientes mutantes mediante deleciones de aminoácidos en pasos de cinco a diez unidades desde la región C-terminal: AIR $\Delta 268, \quad$ AIR $\Delta 273$, AIR $\Delta 282$ AIR $\Delta 290$, AIR $\Delta 299$, AIR $\Delta 307$ y AIR $\Delta 317$. Mediante experimentos de co-transfección en células HEK-293 con HA-LC3A o HA-LC3B para Western blot se pudo comprobar que la secuencia minima para inducir autofagia estaba dentro de la deleción AIR $\Delta 282$. Mientras AIR $\Delta 282$ provoca la conversión de LC3 en niveles muy parecidos a los de la proteína entera, la deleción de cinco aminoácidos más (AIR $\Delta 273)$ bloquea por completo la lipidación de LC3 (figura 18). Este resultado sugiere que los aminoácidos 282-323 de AIR no son necesarios para desencadenar autofagia.

\subsection{La quimera CD16:7-AIR-ID}

Dado que AIR parece ser una proteína transmembrana de tipo I y nuestros ensayos proponen que la parte $\mathrm{N}$-terminal es extracelular y la parte intracelular es el dominio señalizador, sería posible que AIR fuera un receptor capaz de ser estimulado por un potencial ligando que reconociera su dominio extracelular y promoviera la inducción de autofagia a través del dominio intracelular. Desafortunadamente no conocemos ese ligando hipotético. No obstante, en caso de que AIR actuara como receptor, se podría esperar que al ser estimulado adecuadamente, el dominio intracelular fuera suficiente para inducir autofagia. A partir de esta idea se generó la quimera CD16:7AIR-ID que consta de la parte extracelular del receptor CD16 incluyendo el leader que la lleva a la superficie celular, la parte transmembrana que proviene del antígeno de células T CD7 y la parte intracelular de AIR (aa 263-323). Como control negativo se empleó la quimera sin dominio intracelular y, consecuentemente, sin ningún dominio de AIR, que fue denominada CD16:7-Stop. La quimera CD16:7 permite la utilización anticuerpos frente a CD16 como ligandos artificiales (figura 19A).

Mediante la transfección de estas construcciones en células HEK-293 junto con HA-LC3A se pudo determinar que la agregación de la quimera CD16:7 AIR-ID es capaz de inducir la formación de LC3 II tras la estimulación con el anticuerpo frente a CD16 mientras que la quimera control permanece inactiva. Además, la cantidad del LC3 activado dependía de la concentración del anticuerpo usada para la agregación (figura 19B). Tras haber confirmado que la estrategia con la quimera funciona y que el dominio intracelular de AIR es suficiente para inducir autofagia, 
introdujimos en este contexto varias deleciones del dominio activo de AIR. Para la generación de las quimeras CD16:7 nos apoyamos en los resultados obtenidos con los deleciones C-terminales de la proteína nativa. Así, construimos CD16:7-ID-263289 (ver AIR $\Delta 290$ ) y Cd16:7-ID-263-281 (ver AIR $\Delta 282$ ). Adicionalmente, construimos quimeras con deleciones $\mathrm{N}$-terminales: CD16:7-ID-282-323 y CD16:7ID-290-323 en las que añadimos a la quimera la parte intracelular que les falta a las que son completamente activas (CD16:7-ID-263-289 y Cd16:7-ID-263-281). Para averiguar si se podía acortar el dominio intracelular de AIR del lado $\mathrm{N}$-terminal sin perder potencial de inducir autofagia generamos la quimera CD16:7-ID-267-323 y las deleciones C-terminales correspondientes CD16:7-ID-267-289 y Cd16:7-ID267-281 (figura 20). Cómo se puede ver en los Western blot, las deleciones Nterminales más pequeñas (CD16:7-ID-267-323, CD16:7-ID-267-289 y Cd16:7-ID267-281) pierden gran parte de su capacidad de producir autofagia, y las deleciones mayores (CD16:7-ID-282-323 y CD16:7-ID-290-323) apenas inducen formación de LC3II. Curiosamente, las quimeras que incluyen hasta el aminoácido 289 de AIR parecen aumentar el potencial de inducir autofagia. Este resultado es explicable tras la observación de que las quimeras de este tipo se expresan por encima de la media en la membrana plasmática (ver sección 2.8.1. y figura 21B). Sin embargo, consistente con los ensayos realizados con las deleciones Cterminales de la proteína nativa, las quimeras CD16:7-ID-263-281 y CD16:7-ID263-289 conservan todo el potencial de inducir autofagia propio del dominio intracelular entero (figura $21 \mathrm{~A}$ ).

\subsubsection{Presencia de las diferentes quimeras CD16:7-AIR en la membrana plasmática}

Para poder comparar la actividad autofágica de las diferentes quimeras, es importante saber si se expresan a niveles comparables en la membrana plasmática, pues es el lugar de interacción con el ligando artificial. Con el fin de cuantificar su expresión en este compartimiento, células HEK-293 fueron transfectadas con las diferentes quimeras para ser posteriormente teñidos con un anticuerpo conjugado con isotiocianato de fluoresceína (FITC, del inglés fluorescein isothiocyanate) contra CD16 (en condiciones inhibidoras la endocitosis). De este modo, pudimos registrar la florescencia media por citometría de flujo. Así, vimos que las quimeras con las diferentes deleciones del dominio intracelular de AIR presentaban en general niveles 
inferiores de fluorescencia en comparación con el ID entero o la quimera Stop, que tenían valores muy parecidos. No obstante, las quimeras CD16:7-ID-263-290 y CD16:7-ID-267-290 fueron la excepción, pues observamos una mayor cantidad de las mismas en la superficie celular (figura 21B). Debido a que un potencial superior de inducción de autofagia podría ser causado simplemente por una mayor presencia de la quimera relevante en la superficie de la célula, las deleciones que mostraron un nivel de expresión más alto que el dominio intracelular intacto fueron descartadas de los estudios funcionales posteriores. Siguiendo un razonamiento similar, consideramos que los resultados procedentes de quimeras con bajos niveles de expresión son fiables. Si estas proteínas estimulan autofagia al mismo nivel que CD16:7-ID- 263-323 a pesar de su menor presencia, su actividad será subestimada. Aunque puede parecer desfavorable para los estudios del subdominio activo, en el caso de obtener resultados positivos, éstos estarán todavía más fundamentados. Como consecuencia de estos últimos ensayos se escogió la quimera CD16:7-ID-263-281 como herramienta para los experimentos de estudio de la maquinaría implicada en autofagia inducida por AIR. Esta quimera lleva la deleción más grande del dominio intracelular que todavía es capaz de inducir la generación de LC3II a niveles comparables con la parte intracelular entera.

\subsection{Dependencia de autofagia inducida por $A I R$ de la maquinaria autofágica canónica}

Como se ha mencionado en la introducción (ver sección 1.7.1.), la posible implicación de la maquinaria autofágica se puede estudiar a través del efecto inhibitorio de los fármacos wortmanina (WM) o 3-metiladenina (3-MA), que tienen como diana la PI3K-clase3 Vps34. Esta quinasa forma un complejo con la proteína clave de autofagia Beclin1 (ver sección 1.2.2.) y por eso es importante para la formación de autofagosomas. Células HEK-293 se cotransfectaron con HA-LC3 y las quimeras CD16:7-ID-263-323 o CD16:7-ID-263-281, para ser tratados posteriormente con estos inhibidores justo antes de añadir el ligando artificial ( $\alpha$ CD16). Como se puede observar en los ensayos deWestern blot contra HA-LC3, el tratamiento con ambos inhibidores disminuye considerablemente la cantidad de LC3II inducida por ambas quimeras (figura 23). Por lo tanto, este experimento sugiere que la autofagia inducida por AIR en este contexto depende de la maquinaria molecular activadora de autofagia convencional. 


\subsubsection{Silenciamiento de genes centrales de autofagia}

Para fundamentar la idea de que AIR induce autofagia canónica se empleó una herramienta adicional para inhibir este tipo de autofagia: el silenciamiento de Atgs claves para la inducción de autofagia (Atg5, Atg7 y Beclin1). Atg5 y Atg7 juegan un papel importante en los sistemas que llevan a cabo el crecimiento del autofagosoma (ver sección 1.2.3.). Beclin1 forma parte del complejo de proteínas que es importante en los primeros pasos de la formación del autofagosoma (ver sección 1.2.2. y párrafo anterior). Células HEK-293 fueron primero transfectadas con ARNi contra Atg5, Atg7 o Beclin1 y a continuación con las quimeras (junto con el reportero LC3). En este contexto experimental se pudo comprobar que la depleción de Atg5 y Atg7 reducen los niveles de LC3II promovidos por la agregación de las quimeras. Sin embargo, en el caso de Beclin1 la cantidad de LC3II no disminuye a pesar del silenciamiento considerable de Beclin1 que provocaron los ARNis (figura 24). Por lo tanto, la autofagia inducida por AIR parece depender de la presencia de los mediadores de macroautofagia canónica Atg5 y Atg7, mientras Beclin1 parece irrelevante.

\subsection{No se puede asegurar que AIR actúe independientemente de Beclin1}

Recientemente, se ha descrito un tipo de autofagia no canónica que se induce de forma independiente de Beclin1, incluye la modificación lipídica de LC3, es dependiente de Atg7 y está implicada en muerte celular (Scarlatti et al. 2008). En este sentido, encontramos muchas coincidencias con los datos que hemos obtenido en el contexto de AIR. Sin embargo, este tipo de autofagia independiente de Beclin1 tampoco depende de la PI3K-clase3 (Tian et al. 2010), lo que contradice nuestro resultado obtenido con las quimeras en presencia de los inhibidores de la PI3K, 3MA y WM. Un problema de ambos inhibidores es que su actividad no es específica para la PI3K-clase3. Epistáticamente, por encima de mTORC1, hay una PI3Kclase1 con el efecto opuesto sobre autofagia al que induce la PI3K-clase3, lo cual añade confusión cuando se trata de interpretar la actividad de estos inhibidores. No obstante, en el caso de las quimeras CD16:7-AIR, los niveles basales de autofagia detectados en presencia de las drogas y en ausencia del ligando artificial no son elevados, sugiriendo que 3-MA y WM no han actuado inhibiendo la PI3K-clase1. Además, la adición lipídica de LC3 provocada por las quimeras es inhibida por las 
drogas, por lo que los fármacos probablemente inhiban la autofagia a través de la PI3K-clase3. Es decir, el experimento es fiable y, sin embargo, sigue contradiciendo el resultado del knockdown de Beclin1.

Otra posibilidad que podría explicar la adición lipídica del LC3 en ausencia de Beclin1 consistiría en la formación de LC3II ectópico sin génesis de autofagosomas. Este fenómeno ha sido descrito en miocardios con silenciamiento de Beclin1 y en células stem murinas deficientes para Beclin1. En ambos casos la cantidad de LC3 activado que se observa por Western blot no disminuyó con respeto a las muestras de células wildtype, pero mediante microscopía de fluorescencia no se pudieron detectar autofagosomas en las células carentes de Beclin1 (Matsui et al. 2007). Para evaluar si la generación de LC3II inducida por las quimeras CD16:7 en ausencia de Beclin1 promovía la formación de autofagosomas, repetimos el experimento utilizando en este caso el reportero GFP-LC3 para microscopía de fluorescencia, de forma que pudiéramos cuantificar las vesículas formadas por GFPLC3. Desafortunadamente, esta aproximación experimental da lugar a la aparición espontánea de estructuras no identificables y constituidas por GFP-LC3 en los controles negativos, por lo que no fue posible extraer conclusiones de estos experimentos. Parece por tanto que aunque nos sirvan para ensayos de Western blot, las quimeras Cd16:7-AIR no son la herramienta adecuada para una investigación microscópica de las células. Otra manera de investigar si las quimeras inducen formación de autofagosomas en las células Beclin1 knockdown sería comprobar si hay flujo autofágico. Esto se estudia bloqueando la degradación autofágica, por ejemplo mediante el tratamiento con los inhibidores de proteasas E64d y Pepstatina, o un inhibidor de la acidificación lisosomal, Bafilomicina A1 (ver sección 1.7.1.). Si no hay autofagosomas, tampoco hay degradación por autofagia, por lo que los inhibidores de proteasas y Bafilomicina A1 no tendrían la capacidad de aumentar la cantidad del LC3II cuantificado.

En estudios preliminares, la utilización de estas drogas en células transfectadas dio resultados contradictorios, sugiriendo una deficiente optimización del sistema experimental. Por este motivo, no hemos podido elucidar el verdadero papel de Beclin1 en la autofagia inducida por AIR. 


\subsection{El flujo autofágico y AIR}

Es posible que los fármacos utilizados para bloquear el flujo autofágico no sean los más adecuados para nuestro caso. Por un lado, este tipo de reactivos se usa principalmente en autofagia inducida por ayuno y no creemos que éste sea el estímulo principal por el cual se activa AIR. Por otro lado, se aplican durante tiempos más cortos (2-8h) que el ligando artificial (24h). Asimismo, sabemos por experimentos en nuestro laboratorio que la Bafilomicina afecta a la endocitosis de AIR (dato no mostrado). Al inhibir su internalización (y por lo tanto la génesis de LC3II) y el flujo autofágico inducido por esta proteína (y por lo tanto la degradación de LC3II) es probable que los efectos de la Bafilomicina sobre LC3II tiendan a compensarse. Otro escenario posible para explicar los resultados contradictorios con los inhibidores de la degradación en autolisosomas, sería que AIR induce la formación de fagosomas convencionales que son positivos para LC3. Este fenómeno ha sido descrito en un reciente estudio donde se observó la translocación de LC3 a las membranas fagosomales tras la estimulación de TLRs en un proceso dependiente de Atg5 y Atg7, que además mejoró las propiedades bactericidas del fagosoma (Sanjuan et al. 2007). Los datos que hemos obtenido sobre AIR sugieren que AIR pudiera estar involucrado en la formación de este tipo de vesículas, que a diferencia de los autofagosomas, están formadas por una membrana simple. Es importante señalar en este contexto que no se conoce el efecto que los inhibidores de degradación autofágica tienen sobre este tipo de fagosomas.

\subsection{AIR como modulador de APP}

En un estudio reciente AIR ha sido descrito como modulador de la glicosilación compleja de la proteína APP (Ullrich et al. 2010). Está proteína y su procesamiento por las secretasas a y $\beta$ están implicados en la enfermedad de Alzheimer (ver sección 1.3.). Según los autores de este trabajo, AIR se localiza primordialmente en el aparato de Golgi donde tanto su sobreexpresión como el silenciamiento de la proteína inhiben la glicosilación de APP. Esta menor glicosilación disminuye la localización de APP en los compartimentos en donde se encuentran sus secretasas, y por eso su procesamiento decae. Sin embargo, no queda claro qué efecto pudiera tener esta alteración de la glicosilación en la enfermedad de Alzheimer, sobre todo porque AIR afecta al procesamiento por ambas secretasas, la a y la $\beta$. Mientras el 
producto de la modificación de APP por la secretasa a parece ser neuroprotector (Stein et al. 2004), el procesamiento por la secretasa $\beta$ produce el $A \beta$, que es neurotóxico y promueve la pérdida de neuronas (Yankner et al. 1990).

Aunque Ullrich et al. no mencionan la autofagia, se cree que este proceso tiene un efecto beneficioso en la enfermedad de Alzheimer, pues protege a las neuronas mediante la degradación del $A \beta$ tóxico (Boland et al. 2010). Además, un proceso autofágico defectuoso ha sido descrito como probable causa patogénica en este contexto ( $Y u$ et al. 2005). No obstante, no podemos correlacionar los resultados del estudio de Ullrich et al. con los nuestros por diferentes razones. Por un lado, no creemos que AIR esté actuando en el aparato de Golgi puesto que en nuestras manos AIR localiza principalmente en los compartimentos endosomales-lisosomales, y pensamos que este es el lugar dónde actúa. Por otra parte, aunque se ha descrito que el homólogo de AIR, BSMAP, participa en la modulación de la glicolisación del APP, el dominio de AIR que hemos identificado como necesario y suficiente para inducir autofagia, presenta muy poca homología en BSMAP. 


\section{$X$ Conclusions in}

\section{Spanish}

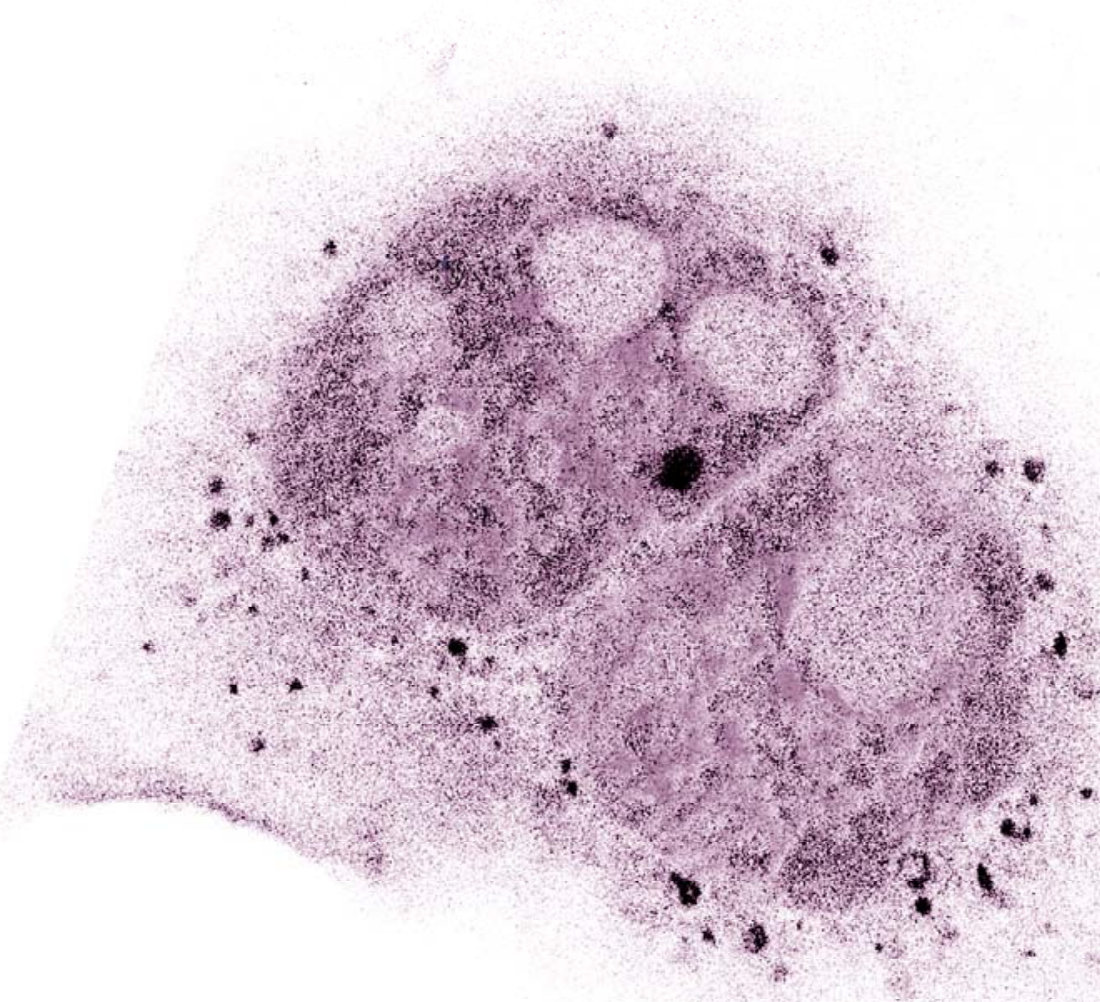





\section{Conclusiones}

1. La sobreexpresión de AIR en células humanas induce autofagia.

2. La sobreexpresión masiva de AIR induce un tipo de muerte que es independiente de caspasas.

3. AIR se expresa en la membrana plasmática y se endocita rápidamente y de forma constante.

4. AIR se localiza preferentemente en los compartimentos endosomaleslisosomales.

5. El dominio $\mathrm{N}$-terminal de AIR es extracelular y el C-terminal es intracelular.

6. El dominio intracelular es necesario y suficiente para inducir autofagia.

7. El subdominio de AIR localizado entre los aminoácidos 263-281 es suficiente para inducir autofagia.

8. La autofagia inducida por AIR depende de la PI3K de clase3, Vps34.

9. La autofagia inducida por AIR depende de Atg5 y Atg7. 



\section{Literature}

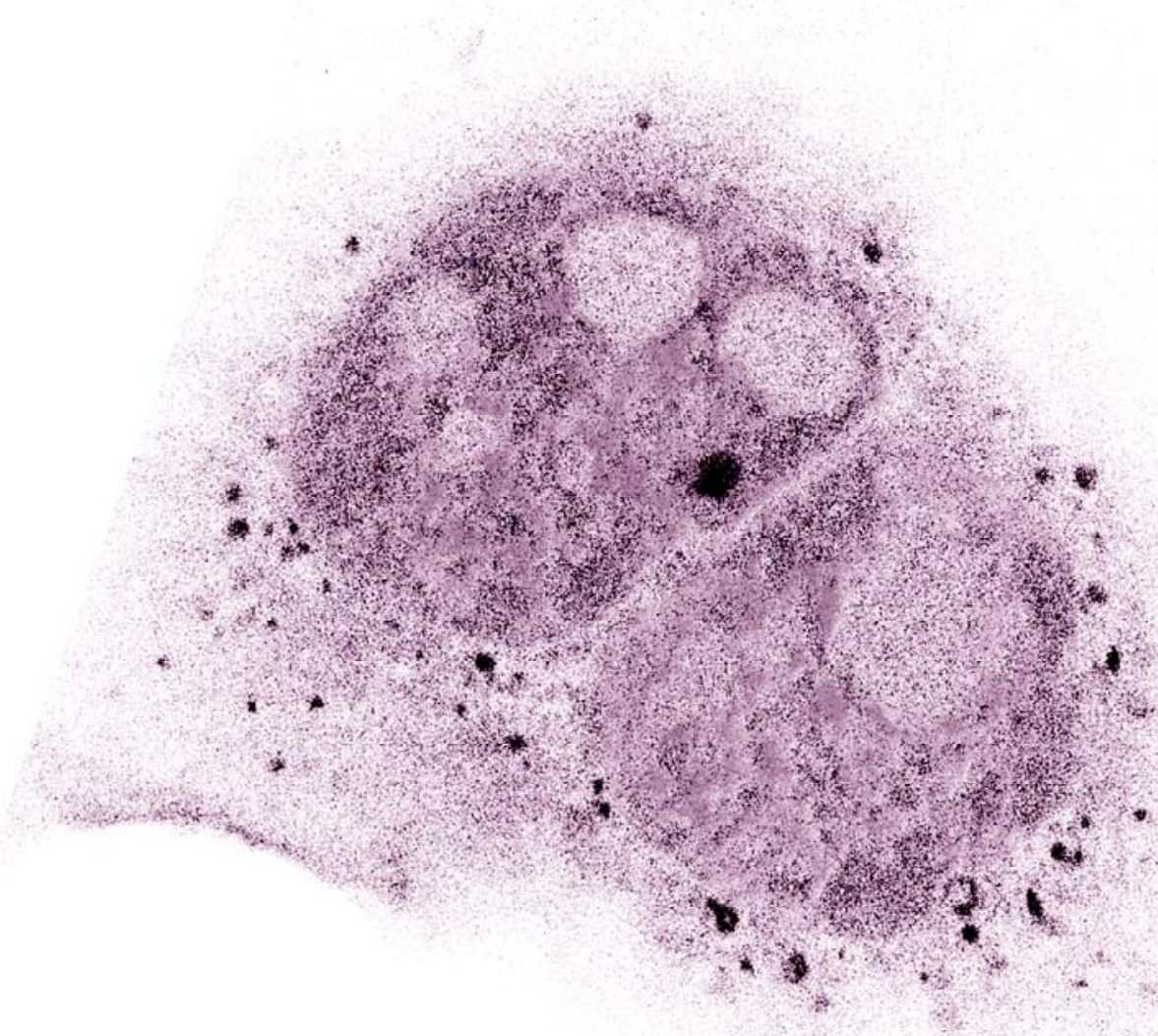



- AKDEMIR, F. FARKAS, R. CHEN, P. JUHASZ, G. MEDVED'OVA, L. SASS, M. WANG, L. WANG, X. CHITTARANJAN, S. GORSKI, S. M. RODRIGUEZ, A. \& ABRAMS, J. M. (2006). Autophagy occurs upstream or parallel to the apoptosome during histolytic cell death. Development 133(8), 1457-1465.

- ALBAYRAK, T. \& GRIMM, S. (2003). A high-throughput screen for single gene activities: isolation of apoptosis inducers. Biochem Biophys Res Commun 304(4), 772-776.

- ALCALA, S. KLEE, M. FERNANDEZ, J. FLEISCHER, A. \& PIMENTEL-MUINOS, F. X. (2008). A highthroughput screening for mammalian cell death effectors identifies the mitochondrial phosphate carrier as a regulator of cytochrome c release. Oncogene 27(1), 44-54.

- amaravadi, R. K. YU, D. LUM, J. J. BUI, T. CHRISTOPHOROU, M. A. EVAN, G. I. THOMASTIKHONENKO, A. \& THOMPSON, C. B. (2007). Autophagy inhibition enhances therapy-induced apoptosis in a Myc-induced model of lymphoma. J Clin Invest 117(2), 326-336.

- ANDREW, S. E. GoldBeRG, Y. P. KREMER, B. TELENIUS, H. THEILMANN, J. ADAM, S. STARR, E. SQUITIERI, F. LIN, B. KALCHMAN, M. A. \& ET AL. (1993). The relationship between trinucleotide (CAG) repeat length and clinical features of Huntington's disease. Nat Genet 4(4), 398-403.

- ARICO, S. Petiot, A. BAUVy, C. DUbBelhuis, P. F. MEIJER, A. J. CODOGNo, P. \& OGIERDENIS, E. (2001). The tumor suppressor PTEN positively regulates macroautophagy by inhibiting the phosphatidylinositol 3-kinase/protein kinase B pathway. J Biol Chem 276(38), 35243-35246.

- ARSTILA, A. U. \& TRUMP, B. F. (1968). Studies on cellular autophagocytosis. The formation of autophagic vacuoles in the liver after glucagon administration. Am J Patho/ 53(5), 687-733.

- BALGI, A. D. FONSECA, B. D. DONOHUE, E. TSANG, T. C. LAJOIE, P. PROUD, C. G. NABI, I. R. \& ROBERGE, M. (2009). Screen for chemical modulators of autophagy reveals novel therapeutic inhibitors of mTORC1 signaling. PLoS One 4(9), e7124.

- BELLODI, C. LIDONNICI, M. R. HAMILTON, A. HELGASON, G. V. SOlIERA, A. R. RONCHETTI, M. GALAVOTTI, S. YOUNG, K. W. SELMI, T. YACOBI, R. VAN ETTEN, R. A. DONATO, N. HUNTER, A. DINSDALE, D. TIRRO, E. VIGNERI, P. NICOTERA, P. DYER, M. J. HOLYOAKE, T. SALOMONI, P. \& CALABRETTA, B. (2009). Targeting autophagy potentiates tyrosine kinase inhibitor-induced cell death in Philadelphia chromosome-positive cells, including primary CML stem cells. J Clin Invest 119(5), 11091123.

- BERRY, D. L. \& BAEHRECKE, E. H. (2007). Growth arrest and autophagy are required for salivary gland cell degradation in Drosophila. Cel/ 131(6), 1137-1148.

- BETIN, V. M. \& LANE, J. D. (2009). Caspase cleavage of Atg4D stimulates GABARAP-L1 processing and triggers mitochondrial targeting and apoptosis. J Cell Sci 122(Pt 14), 2554-2566.

- BHOJ, V. G. \& CHEN, Z. J. (2009). Ubiquitylation in innate and adaptive immunity. Nature 458(7237), 430-437.

- BIRMINGHAM, C. L. SMITH, A. C. BAKOWSKI, M. A. YOSHIMORI, T. \& BRUMELL, J. H. (2006). Autophagy controls Salmonella infection in response to damage to the Salmonella-containing vacuole. $J$ Biol Chem 281(16), 11374-11383.

- BJoRkoy, G. LamaRK, T. PANKIV, S. OVERVATN, A. BRECH, A. \& JOHANSEN, T. (2009). Monitoring autophagic degradation of p62/SQSTM1. Methods Enzymo/ 452, 181-197.

- BLANDER, J. M. \& MEDZHITOV, R. (2004). Regulation of phagosome maturation by signals from tolllike receptors. Science 304(5673), 1014-1018.

- BLOMmAART, E. F. KRAUSE, U. SCHELLENS, J. P. VREELING-SINDELAROVA, H. \& MEIJER, A. J. (1997). The phosphatidylinositol 3-kinase inhibitors wortmannin and LY294002 inhibit autophagy in isolated rat hepatocytes. Eur J Biochem 243(1-2), 240-246.

- BOISE, L. H. GONZALEZ-GARCIA, M. POSTEMA, C. E. DING, L. LINDSTEN, T. TURKA, L. A. MAO, X. NUNEZ, G. \& THOMPSON, C. B. (1993). bcl-x, a bcl-2-related gene that functions as a dominant regulator of apoptotic cell death. Cell 74(4), 597-608.

- BOLAND, B. SMITH, D. A. MOONEY, D. JUNG, S. S. WALSH, D. M. \& PLATT, F. M. (2010). Macroautophagy is not directly involved in the metabolism of amyloid precursor protein. $J$ Biol Chem 285(48), 37415-37426. 
- BUDANOV, A. V. \& KARIN, M. (2008). p53 target genes sestrin1 and sestrin2 connect genotoxic stress and mTOR signaling. Cel/ 134(3), 451-460.

- CADWELL, K. LIU, J. Y. BROWN, S. L. MIYOSHI, H. LOH, J. LENNERZ, J. K. KISHI, C. KC, W. CARRERO, J. A. HUNT, S. STONE, C. D. BRUNT, E. M. XAVIER, R. J. SLECKMAN, B. P. LI, E. MIZUSHIMA, N. STAPPENBECK, T. S. \& VIRGIN, H. W. T. (2008). A key role for autophagy and the autophagy gene Atg1611 in mouse and human intestinal Paneth cells. Nature 456(7219), 259-263.

- CALLUS, B. A. \& VAUX, D. L. (2007). Caspase inhibitors: viral, cellular and chemical. Cell Death Differ 14(1), 73-78.

- CANN, G. M. GUIGNABERT, C. YING, L. DESHPANDE, N. BEKKER, J. M. WANG, L. ZHOU, B. \& RABINOVITCH, M. (2008). Developmental expression of LC3alpha and beta: absence of fibronectin or autophagy phenotype in LC3beta knockout mice. Dev Dyn 237(1), 187-195.

- CAREW, J. S. NAWROCKI, S. T. KAHUE, C. N. ZHANG, H. YANG, C. CHUNG, L. HOUGHTON, J. A. HUANG, P. GILES, F. J. \& CLEVELAND, J. L. (2007). Targeting autophagy augments the anticancer activity of the histone deacetylase inhibitor SAHA to overcome Bcr-Abl-mediated drug resistance. Blood 110(1), 313-322.

- CARO, L. H. Plomp, P. J. WOlvetANG, E. J. KERKHOF, C. \& MEIJER, A. J. (1988). 3Methyladenine, an inhibitor of autophagy, has multiple effects on metabolism. Eur $J$ Biochem 175(2), 325-329.

- CECCONI, F. \& LEVINE, B. (2008). The role of autophagy in mammalian development: cell makeover rather than cell death. Dev Cel/ 15(3), 344-357.

- Citron, m. Westaway, D. XIA, W. CARLSON, G. DIEHL, T. LEVESQUE, G. JOHNSON-WOOD, K. LEE, M. SEUBERT, P. DAVIS, A. KHOLODENKO, D. MOTTER, R. SHERRINGTON, R. PERRY, B. YAO, H. STROME, R. LIEBERBURG, I. ROMMENS, J. KIM, S. SCHENK, D. FRASER, P. ST GEORGE HYSLOP, P. \& SELKOE, D. J. (1997). Mutant presenilins of Alzheimer's disease increase production of 42-residue amyloid beta-protein in both transfected cells and transgenic mice. Nat Med 3(1), 67-72.

- CLEM, R. J. \& MILLER, L. K. (1993). Apoptosis reduces both the in vitro replication and the in vivo infectivity of a baculovirus. J Virol 67(7), 3730-3738.

- COHEN, G. M. SUN, X. M. FEARNHEAD, H. MACFARLANE, M. BROWN, D. G. SNOWDEN, R. T. \& DINSDALE, D. (1994). Formation of large molecular weight fragments of DNA is a key committed step of apoptosis in thymocytes. J Immuno/ 153(2), 507-516.

- CRIGHTON, D. WILKINSON, S. O'PREY, J. SYED, N. SMITH, P. HARRISON, P. R. GASCO, M. GARRONE, O. CROOK, T. \& RYAN, K. M. (2006). DRAM, a p53-induced modulator of autophagy, is critical for apoptosis. Cel/ 126(1), 121-134.

- CUERVO, A. M. (2008). Autophagy and aging: keeping that old broom working. Trends Genet 24(12), 604-612.

- CUERVO, A. M. \& DICE, J. F. (2000a). Age-related decline in chaperone-mediated autophagy. J Biol Chem 275(40), 31505-31513.

- CUERVO, A. M. \& DICE, J. F. (2000b). Regulation of lamp2a levels in the lysosomal membrane. Traffic 1(7), 570-583.

- CueRVo, A. M. STEFANIS, L. FREDENBURG, R. LANSBURY, P. T. \& SULZER, D. (2004). Impaired degradation of mutant alpha-synuclein by chaperone-mediated autophagy. Science 305(5688), 12921295.

- CHAN, E. Y. LONGATTI, A. MCKNIGHT, N. C. \& TOOZE, S. A. (2009). Kinase-inactivated ULK proteins inhibit autophagy via their conserved C-terminal domains using an Atg13-independent mechanism. Mol Cell Biol 29(1), 157-171.

- CHANDA, S. K. WHITE, S. ORTH, A. P. REISDORPH, R. MIRAGLIA, L. THOMAS, R. S. DEJESUS, P. MASON, D. E. HUANG, Q. VEGA, R. YU, D. H. NELSON, C. G. SMITH, B. M. TERRY, R. LINFORD, A. S. YU, Y. CHIRN, G. W. SONG, C. LABOW, M. A. COHEN, D. KING, F. J. PETERS, E. C. SCHULTZ, P. G. VOGT, P. K. HOGENESCH, J. B. \& CALDWELL, J. S. (2003). Genome-scale functional profiling of the mammalian AP-1 signaling pathway. Proc Natl Acad Sci U S A 100(21), 12153-12158. 
- CHIPUK, J. E. KUWANA, T. BOUCHIER-HAYES, L. DROIN, N. M. NEWMEYER, D. D. SCHULER, M. \& GREEN, D. R. (2004). Direct activation of Bax by p53 mediates mitochondrial membrane permeabilization and apoptosis. Science 303(5660), 1010-1014.

- CHITTENDEN, T. HARRINGTON, E. A. O'CONNOR, R. FLEMINGTON, C. LUTZ, R. J. EVAN, G. I. \& GUILD, B. C. (1995). Induction of apoptosis by the Bcl-2 homologue Bak. Nature 374(6524), 733736.

- DANIAL, N. N. \& KORSMEYER, S. J. (2004). Cell death: critical control points. Cel/116(2), 205-219.

- davies, S. W. turmaine, M. COZens, B. A. Difiglia, M. Sharp, A. H. Ross, C. A. SCHERZINGER, E. WANKER, E. E. MANGIARINI, L. \& BATES, G. P. (1997). Formation of neuronal intranuclear inclusions underlies the neurological dysfunction in mice transgenic for the HD mutation. Cell 90(3), 537-548.

- DE DUVE, C. PRESSman, B. C. GIANetTo, R. WATtiauX, R. \& APPELMANS, F. (1955). Tissue fractionation studies. 6. Intracellular distribution patterns of enzymes in rat-liver tissue. Biochem $J 60(4)$, 604-617.

- DEBNATH, J. BAEHRECKE, E. H. \& KROEMER, G. (2005). Does autophagy contribute to cell death? Autophagy 1(2), 66-74.

- DEgENHARDT, K. MATHEW, R. BEAUdoIN, B. BRAY, K. ANDERSON, D. CHEN, G. MUKHERJEe, C. SHI, Y. GELINAS, C. FAN, Y. NELSON, D. A. JIN, S. \& WHITE, E. (2006). Autophagy promotes tumor cell survival and restricts necrosis, inflammation, and tumorigenesis. Cancer Cel/ 10(1), 51-64.

- DELBRIDGE, L. M. \& O'RIORDAN, M. X. (2007). Innate recognition of intracellular bacteria. Curr Opin Immuno/ 19(1), 10-16.

- DELGAdo, M. A. ELMAOUED, R. A. DAVIS, A. S. KYEI, G. \& DERETIC, V. (2008). Toll-like receptors control autophagy. Embo J 27(7), 1110-1121.

- DERETIC, V. (2009). Multiple regulatory and effector roles of autophagy in immunity. Curr Opin Immunol 21(1), 53-62.

- DERETIC, V. (2010). Autophagy in infection. Curr Opin Cell Bio/ 22(2), 252-262.

- DICE, J. F. TERLECKY, S. R. CHIANG, H. L. OLSON, T. S. ISENMAN, L. D. SHORT-RUSSELL, S. R. FREUNDLIEB, S. \& TERLECKY, L. J. (1990). A selective pathway for degradation of cytosolic proteins by lysosomes. Semin Cell Biol 1(6), 449-455.

- DIEBOlD, S. S. KAISHO, T. HEMMI, H. AKIRA, S. \& REIS E SOUSA, C. (2004). Innate antiviral responses by means of TLR7-mediated recognition of single-stranded RNA. Science 303(5663), 15291531.

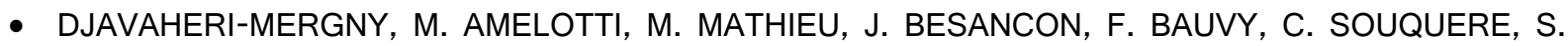
PIERRON, G. \& CODOGNO, P. (2006). NF-kappaB activation represses tumor necrosis factor-alphainduced autophagy. J Biol Chem 281(41), 30373-30382.

- Dubouloz, F. DELOChe, O. WANKE, V. CAMERONI, E. \& DE VIRGILIO, C. (2005). The TOR and EGO protein complexes orchestrate microautophagy in yeast. Mol Cel/ 19(1), 15-26.

- EDINGER, A. L. \& THOMPSON, C. B. (2004). Death by design: apoptosis, necrosis and autophagy. Curr Opin Cell Biol 16(6), 663-669.

- ELGENDY, M. SHERIDAN, C. BRUMATTI, G. \& MARTIN, S. J. (2011). Oncogenic Ras-Induced Expression of Noxa and Beclin-1 Promotes Autophagic Cell Death and Limits Clonogenic Survival. Mol Cell.

- ELSON, G. C. DE COIGNAC, A. B. AUBRY, J. P. DELNESTE, Y. MAGISTRELLI, G. HOLZWARTH, J. BONNEFOY, J. Y. \& GAUCHAT, J. F. (1999). BSMAP, a novel protein expressed specifically in the brain whose gene is localized on chromosome 19p12. Biochem Biophys Res Commun 264(1), 55-62.

- ENGLish, L. CHEMALI, M. DURON, J. RONDEAU, C. LAPLANTE, A. GINGRAS, D. ALEXANDER, D. LEIB, D. NORBURY, C. LIPPE, R. \& DESJARDINS, M. (2009). Autophagy enhances the presentation of endogenous viral antigens on MHC class I molecules during HSV-1 infection. Nat Immuno/ 10(5), 480-487. 
- esclatine, A. ChaumorCel, M. \& CODOGNO, P. (2009). Macroautophagy signaling and regulation. Curr Top Microbiol Immunol 335, 33-70.

- ESKELINEN, E. L. (2006). Roles of LAMP-1 and LAMP-2 in lysosome biogenesis and autophagy. Mol Aspects Med 27(5-6), 495-502.

- ESPERT, L. DENIZOT, M. GRIMALDI, M. ROBERT-HEBMANN, V. GAY, B. VARBANOV, M. CODOGNO, P. \& BIARD-PIECHACZYK, M. (2006). Autophagy is involved in T cell death after binding of HIV-1 envelope proteins to CXCR4. J Clin Invest 116(8), 2161-2172.

- FIMIA, G. M. \& PIACENTINI, M. (2010). Regulation of autophagy in mammals and its interplay with apoptosis. Cell Mol Life Sci 67(10), 1581-1588.

- fimia, G. M. StOYkova, A. ROMAGNOl, A. GIUNTA, L. DI BARTOlOMEO, S. NARDACCI, R. CORAZZARI, M. FUOCO, C. UCAR, A. SCHWARTZ, P. GRUSS, P. PIACENTINI, M. CHOWDHURY, K. \& CECCONI, F. (2007). Ambra1 regulates autophagy and development of the nervous system. Nature $447(7148), 1121-1125$.

- FRANGIONI, J. V. \& NEEL, B. G. (1993). Solubilization and purification of enzymatically active glutathione S-transferase (pGEX) fusion proteins. Anal Biochem 210(1), 179-187.

- FUJITA, N. SAITOH, T. KAGEYAMA, S. AKIRA, S. NODA, T. \& YOSHIMORI, T. (2009). Differential involvement of Atg16L1 in Crohn disease and canonical autophagy: analysis of the organization of the Atg16L1 complex in fibroblasts. $J$ Biol Chem 284(47), 32602-32609.

- FUKAZAWA, I. \& KOYAMA, J. (1982). Effects of various protease inhibitors on phagocytosis of antigenantibody complex by macrophages. J Pharmacobiodyn 5(9), 699-707.

- FURUYA, N. YU, J. BYFIELD, M. PATTINGRE, S. \& LEVINE, B. (2005). The evolutionarily conserved domain of Beclin 1 is required for Vps34 binding, autophagy and tumor suppressor function. Autophagy 1(1), 46-52.

- GANLEY, I. G. LAM DU, H. WANG, J. DING, X. CHEN, S. \& JIANG, X. (2009). ULK1.ATG13.FIP200 complex mediates mTOR signaling and is essential for autophagy. J Biol Chem 284(18), 12297-12305.

- GANNAGE, M. \& MUNZ, C. (2009). Autophagy in MHC class II presentation of endogenous antigens. Curr Top Microbiol Immunol 335, 123-140.

- GOLDBERG, A. L. (2003). Protein degradation and protection against misfolded or damaged proteins. Nature 426(6968), 895-899.

- GOZUACIK, D. BIALIK, S. RAVEH, T. MITOU, G. SHOHAT, G. SABANAY, H. MIZUSHIMA, N. YOSHIMORI, T. \& KIMCHI, A. (2008). DAP-kinase is a mediator of endoplasmic reticulum stressinduced caspase activation and autophagic cell death. Cell Death Differ 15(12), 1875-1886.

- Grotemeier, A. Alers, S. Pfisterer, S. G. PAAsch, F. DAUbrawa, M. Dieterle, A. VIOLLET, B. WESSELBORG, S. PROIKAS-CEZANNE, T. \& STORK, B. (2010). AMPK-independent induction of autophagy by cytosolic Ca2 + increase. Cell Signal 22(6), 914-925.

- GUtieRrez, M. G. MASTER, S. S. SINGH, S. B. TAYLOR, G. A. COLOMBO, M. I. \& DERETIC, V. (2004a). Autophagy is a defense mechanism inhibiting BCG and Mycobacterium tuberculosis survival in infected macrophages. Cel/119(6), 753-766.

- GUTIERREZ, M. G. MUNAFO, D. B. BERON, W. \& COLOMBO, M. I. (2004b). Rab7 is required for the normal progression of the autophagic pathway in mammalian cells. J Cell Sci 117(Pt 13), 2687-2697.

- HAILEY, D. W. RAMBOLD, A. S. SATPUTE-KRISHNAN, P. MITRA, K. SOUGRAT, R. KIM, P. K. \& LIPPINCOTT-SCHWARTZ, J. (2010). Mitochondria supply membranes for autophagosome biogenesis during starvation. Cel/ 141(4), 656-667.

- hampe, J. franke, A. ROSenstiel, P. till, A. TEUber, M. HUSE, K. AlBReCht, M. MAYR, G. DE LA VEGA, F. M. BRIGGS, J. GUNTHER, S. PRESCOTT, N. J. ONNIE, C. M. HASLER, R. SIPOS, B. FOLSCH, U. R. LENGAUER, T. PLATZER, M. MATHEW, C. G. KRAWCZAK, M. \& SCHREIBER, S. (2007). A genome-wide association scan of nonsynonymous SNPs identifies a susceptibility variant for Crohn disease in ATG16L1. Nat Genet 39(2), 207-211.

- HANSEN, M. CHANDRA, A. MITIC, L. L. ONKEN, B. DRISCOLL, M. \& KENYON, C. (2008). A role for autophagy in the extension of lifespan by dietary restriction in C. elegans. PLoS Genet 4(2), e24. 
- haRA, T. TAKAMURA, A. KISHI, C. IEMURA, S. NATSUME, T. GUAN, J. L. \& MizUSHIMA, N. (2008). FIP200, a ULK-interacting protein, is required for autophagosome formation in mammalian cells. J Cell Biol 181(3), 497-510.

- hARRIS, J. DE hARO, S. A. MASTER, S. S. KEANE, J. ROBERTS, E. A. DELGADO, M. \& DERETIC, V. (2007). T helper 2 cytokines inhibit autophagic control of intracellular Mycobacterium tuberculosis. Immunity 27(3), 505-517.

- HAYASHI-NISHINO, M. FUJITA, N. NODA, T. YAMAGUCHI, A. YOSHIMORI, T. \& YAMAMOTO, A. (2009). A subdomain of the endoplasmic reticulum forms a cradle for autophagosome formation. Nat Cell Biol 11(12), 1433-1437.

- HE, H. DANG, Y. DAI, F. GUO, Z. WU, J. SHE, X. PEI, Y. CHEN, Y. LING, W. WU, C. ZHAO, S. LIU, J. O. \& YU, L. (2003). Post-translational modifications of three members of the human MAP1LC3 family and detection of a novel type of modification for MAP1LC3B. J Biol Chem 278(31), 29278-29287.

- HEIL, F. HEMMI, H. HOCHREIN, H. AMPENBERGER, F. KIRSCHNING, C. AKIRA, S. LIPFORD, G. WAGNER, H. \& BAUER, S. (2004). Species-specific recognition of single-stranded RNA via toll-like receptor 7 and 8. Science 303(5663), 1526-1529.

- HOTCHKISS, R. S. STRASSER, A. MCDUNN, J. E. \& SWANSON, P. E. (2009). Cell death. N Engl J Med 361(16), 1570-1583.

- HOYER-HANSEN, M. BASTHOLM, L. SZYNIAROWSKI, P. CAMPANELLA, M. SZABADKAI, G. FARKAS, T. BIANCHI, K. FEHRENBACHER, N. ELLING, F. RIZZUTO, R. MATHIASEN, I. S. \& JAATTELA, M. (2007). Control of macroautophagy by calcium, calmodulin-dependent kinase kinasebeta, and $\mathrm{Bcl}-2$. Mol Cel/ 25(2), 193-205.

- HUTCHINS, M. U. \& KLIONSKY, D. J. (2001). Vacuolar localization of oligomeric alpha-mannosidase requires the cytoplasm to vacuole targeting and autophagy pathway components in Saccharomyces cerevisiae. J Biol Chem 276(23), 20491-20498.

- HUYNH, K. K. ESKELINEN, E. L. SCOTT, C. C. MALEVANETS, A. SAFTIG, P. \& GRINSTEIN, S. (2007). LAMP proteins are required for fusion of lysosomes with phagosomes. Embo J 26(2), 313-324.

- ICHIMURA, Y. KIRISAKO, T. TAKAO, T. SATOMI, Y. SHIMONISHI, Y. ISHIHARA, N. MIZUSHIMA, N. TANIDA, I. KOMINAMI, E. OHSUMI, M. NODA, T. \& OHSUMI, Y. (2000). A ubiquitin-like system mediates protein lipidation. Nature 408(6811), 488-492.

- INOUE, H. NOJIMA, H. \& OKAYAMA, H. (1990). High efficiency transformation of Escherichia coli with plasmids. Gene 96(1), 23-28.

- INOUE, Y. \& KLIONSKY, D. J. (2010). Regulation of macroautophagy in Saccharomyces cerevisiae. Semin Cell Dev Biol.

- JAHREISS, L. MENZIES, F. M. \& RUBINSZTEIN, D. C. (2008). The itinerary of autophagosomes: from peripheral formation to kiss-and-run fusion with lysosomes. Traffic 9(4), 574-587.

- KABEYA, Y. MIZUSHIMA, N. UENO, T. YAMAMOTO, A. KIRISAKO, T. NODA, T. KOMINAMI, E. OHSUMI, Y. \& YOSHIMORI, T. (2000). LC3, a mammalian homologue of yeast Apg8p, is localized in autophagosome membranes after processing. Embo J 19(21), 5720-5728.

- KAMADA, Y. FUNAKOSHI, T. SHINTANI, T. NAGANO, K. OHSUMI, M. \& OHSUMI, Y. (2000). Tormediated induction of autophagy via an Apg1 protein kinase complex. J Cell Bio/ 150(6), 1507-1513.

- KANZAWA, T. GERMANO, I. M. KOMATA, T. ITO, H. KONDO, Y. \& KONDO, S. (2004). Role of autophagy in temozolomide-induced cytotoxicity for malignant glioma cells. Cell Death Differ 11(4), 448457.

- KANZAWA, T. ZHANG, L. XIAO, L. GERMANO, I. M. KONDO, Y. \& KONDO, S. (2005). Arsenic trioxide induces autophagic cell death in malignant glioma cells by upregulation of mitochondrial cell death protein BNIP3. Oncogene 24(6), 980-991.

- KATAYAMA, M. KAWAGUCHI, T. BERGER, M. S. \& PIEPER, R. O. (2007). DNA damaging agentinduced autophagy produces a cytoprotective adenosine triphosphate surge in malignant glioma cells. Cell Death Differ 14(3), 548-558. 
- KAUSHIK, S. MASSEY, A. C. MIZUSHIMA, N. \& CUERVO, A. M. (2008). Constitutive activation of chaperone-mediated autophagy in cells with impaired macroautophagy. Mol Biol Cell 19(5), 2179-2192.

- KERR, J. F. WYLLIE, A. H. \& CURRIE, A. R. (1972). Apoptosis: a basic biological phenomenon with wide-ranging implications in tissue kinetics. Br J Cancer 26(4), 239-257.

- KIEFER, M. C. BRAUER, M. J. POWERS, V. C. WU, J. J. UMANSKY, S. R. TOMEI, L. D. \& BARR, P. J. (1995). Modulation of apoptosis by the widely distributed Bcl-2 homologue Bak. Nature 374(6524), 736-739.

- KIHARA, A. KABEYA, Y. OHSUMI, Y. \& YOSHIMORI, T. (2001). Beclin-phosphatidylinositol 3-kinase complex functions at the trans-Golgi network. EMBO Rep 2(4), 330-335.

- KIM, K. W. mUtTer, R. W. CAO, C. AlbeRT, J. M. FREemAN, M. hALLAHAN, D. E. \& LU, B. (2006). Autophagy for cancer therapy through inhibition of pro-apoptotic proteins and mammalian target of rapamycin signaling. $J$ Biol Chem 281(48), 36883-36890.

- KIMURA, S. NODA, T. \& YOSHIMORI, T. (2008). Dynein-dependent movement of autophagosomes mediates efficient encounters with lysosomes. Cell Struct Funct 33(1), 109-122.

- KLEE, M. (2008). Caracterización funcional de la molécula proapoptótica Bak en el retículo endoplasmático.

- KLIONSKY, D. J. ELAZAR, Z. SEGLEN, P. O. \& RUBINSZTEIN, D. C. (2008). Does bafilomycin A1 block the fusion of autophagosomes with lysosomes? Autophagy 4(7), 849-950.

- KOIKE, M. NAKANISHI, H. SAFTIG, P. EZAKI, J. ISAHARA, K. OHSAWA, Y. SCHULZ-SCHAEFFER, W. WATANABE, T. WAGURI, S. KAMETAKA, S. SHIBATA, M. YAMAMOTO, K. KOMINAMI, E. PETERS, C. VON FIGURA, K. \& UCHIYAMA, Y. (2000). Cathepsin D deficiency induces lysosomal storage with ceroid lipofuscin in mouse CNS neurons. J Neurosci 20(18), 6898-6906.

- KOMATSU, M. KUROKAWA, H. WAGURI, S. TAGUCHI, K. KOBAYASHI, A. ICHIMURA, Y. SOU, Y. S. UENO, I. SAKAMOTO, A. TONG, K. I. KIM, M. NISHITO, Y. IEMURA, S. NATSUME, T. UENO, T. KOMINAMI, E. MOTOHASHI, H. TANAKA, K. \& YAMAMOTO, M. (2010). The selective autophagy substrate p62 activates the stress responsive transcription factor Nrf2 through inactivation of Keap1. Nat Cell Biol 12(3), 213-223.

- KOMATSU, M. WAGURI, S. CHIBA, T. MURATA, S. IWATA, J. TANIDA, I. UENO, T. KOIKE, M. UCHIYAMA, Y. KOMINAMI, E. \& TANAKA, K. (2006). Loss of autophagy in the central nervous system causes neurodegeneration in mice. Nature 441(7095), 880-884.

- KOMATSU, M. WAGURI, S. UENO, T. IWATA, J. MURATA, S. TANIDA, I. EZAKI, J. MIZUSHIMA, N. OHSUMI, Y. UCHIYAMA, Y. KOMINAMI, E. TANAKA, K. \& CHIBA, T. (2005). Impairment of starvationinduced and constitutive autophagy in Atg7-deficient mice. J Cell Biol 169(3), 425-434.

- KON, M. \& CUERVO, A. M. (2010). Chaperone-mediated autophagy in health and disease. FEBS Lett 584(7), 1399-1404.

- KROEMER, G. \& LEVINE, B. (2008). Autophagic cell death: the story of a misnomer. Nat Rev Mol Cell $\mathrm{Biol}$ 9(12), 1004-1010.

- KRUG, A. LUKER, G. D. BARChET, W. LeIB, D. A. AKIRA, S. \& COLONNA, M. (2004). Herpes simplex virus type 1 activates murine natural interferon-producing cells through toll-like receptor 9. Blood 103(4), 1433-1437.

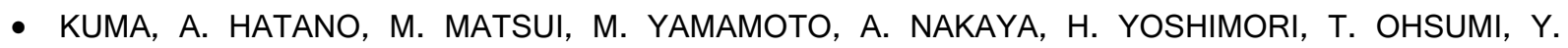
TOKUHISA, T. \& MIZUSHIMA, N. (2004). The role of autophagy during the early neonatal starvation period. Nature 432(7020), 1032-1036.

- KUMA, A. MATSUI, M. \& MIZUSHIMA, N. (2007). LC3, an autophagosome marker, can be incorporated into protein aggregates independent of autophagy: caution in the interpretation of LC3 localization. Autophagy 3(4), 323-328.

- KUWANA, T. BOUCHIER-HAYES, L. CHIPUK, J. E. BONZON, C. SULLIVAN, B. A. GREEN, D. R. \& NEWMEYER, D. D. (2005). BH3 domains of BH3-only proteins differentially regulate Bax-mediated mitochondrial membrane permeabilization both directly and indirectly. Mol Cel/ 17(4), 525-535. 
- LAderoute, K. R. Amin, K. CAlaoAgan, J. M. KNAPP, M. LE, T. ORDUNA, J. FORETZ, M. \& VIOLLET, B. (2006). 5'-AMP-activated protein kinase (AMPK) is induced by low-oxygen and glucose deprivation conditions found in solid-tumor microenvironments. Mol Cell Biol 26(14), 5336-5347.

- LAKHANI, S. A. MASUD, A. KUIDA, K. PORTER, G. A., JR. BOOTH, C. J. MEHAL, W. Z. INAYAT, I. \& FLAVELL, R. A. (2006). Caspases 3 and 7: key mediators of mitochondrial events of apoptosis. Science 311(5762), 847-851.

- LAMKANFI, M. \& DIXIT, V. M. (2009). Inflammasomes: guardians of cytosolic sanctity. Immunol Rev 227(1), 95-105.

- LEE, H. K. LUND, J. M. RAMANATHAN, B. MIZUShIMA, N. \& IWASAKI, A. (2007a). Autophagydependent viral recognition by plasmacytoid dendritic cells. Science 315(5817), 1398-1401.

- LeE, J. H. YU, W. H. KUMAR, A. Lee, S. MOHAN, P. S. PETERHOFf, C. M. WOLfE, D. M. MARTINEZ-VICENTE, M. MASSEY, A. C. SOVAK, G. UCHIYAMA, Y. WESTAWAY, D. CUERVO, A. M. \& NIXON, R. A. (2010). Lysosomal proteolysis and autophagy require presenilin 1 and are disrupted by Alzheimer-related PS1 mutations. Cell 141(7), 1146-1158.

- LEE, S. B. KIM, S. LEE, J. PARK, J. LEE, G. KIM, Y. KIM, J. M. \& CHUNG, J. (2007b). ATG1, an autophagy regulator, inhibits cell growth by negatively regulating $S 6$ kinase. EMBO Rep 8(4), 360-365.

- LEMASTERS, J. J. (2005). Selective mitochondrial autophagy, or mitophagy, as a targeted defense against oxidative stress, mitochondrial dysfunction, and aging. Rejuvenation Res 8(1), 3-5.

- LEVINE, B. (2005). Eating oneself and uninvited guests: autophagy-related pathways in cellular defense. Cell 120(2), 159-162.

- LeVINE, B. \& KROEMER, G. (2008). Autophagy in the pathogenesis of disease. Cel/ 132(1), 27-42.

- LI, J. YeN, C. LIAW, D. PODSypaninA, K. BOSE, S. WANG, S. I. PUC, J. MILIARESIS, C. RODGERS, L. MCCOMBIE, R. BIGNER, S. H. GIOVANELLA, B. C. ITTMANN, M. TYCKO, B. HIBSHOOSH, H. WIGLER, M. H. \& PARSONS, R. (1997). PTEN, a putative protein tyrosine phosphatase gene mutated in human brain, breast, and prostate cancer. Science 275(5308), 19431947.

- LIANG, C. FENG, P. KU, B. DOTAN, I. CANAANI, D. OH, B. H. \& JUNG, J. U. (2006). Autophagic and tumour suppressor activity of a novel Beclin1-binding protein UVRAG. Nat Cell Biol 8(7), 688-699.

- LIANG, C. C. WANG, C. PENG, X. GAN, B. \& GUAN, J. L. (2010). Neural-specific deletion of FIP200 leads to cerebellar degeneration caused by increased neuronal death and axon degeneration. $J$ Biol Chem 285(5), 3499-3509.

- LIANG, X. H. JACKSON, S. SEAMAN, M. BROWN, K. KEMPKES, B. HIBSHOOSH, H. \& LEVINE, B. (1999). Induction of autophagy and inhibition of tumorigenesis by beclin 1. Nature 402(6762), 672676.

- LIANG, X. H. KLEEMAN, L. K. JIANG, H. H. GORDON, G. GOldMAN, J. E. BERRY, G. HERMAN, B. \& LEVINE, B. (1998). Protection against fatal Sindbis virus encephalitis by beclin, a novel Bcl-2interacting protein. J Virol 72(11), 8586-8596.

- LIANG, X. H. YU, J. BROWN, K. \& LEVINE, B. (2001). Beclin 1 contains a leucine-rich nuclear export signal that is required for its autophagy and tumor suppressor function. Cancer Res 61(8), 3443-3449.

- LING, Y. M. SHAW, M. H. AYALA, C. COPPENS, I. TAYLOR, G. A. FERGUSON, D. J. \& YAP, G. S. (2006). Vacuolar and plasma membrane stripping and autophagic elimination of Toxoplasma gondii in primed effector macrophages. J Exp Med 203(9), 2063-2071.

- LIPPINCOTT-SCHWARTZ, J. \& FAMBROUGH, D. M. (1987). Cycling of the integral membrane glycoprotein, LEP100, between plasma membrane and lysosomes: kinetic and morphological analysis. Cell 49(5), 669-677.

- LOWRY, O. H. ROSEBROUGH, N. J. FARR, A. L. \& RANDALL, R. J. (1951). Protein measurement with the Folin phenol reagent. $J$ Biol Chem 193(1), 265-275.

- LUO, S. \& RUBINSZTEIN, D. C. (2010). Apoptosis blocks Beclin 1-dependent autophagosome synthesis: an effect rescued by Bcl-xL. Cell Death Differ 17(2), 268-277. 
- maiuri, M. C. le tOUMElin, G. CRIOllo, A. RAIN, J. C. GAUTIER, F. JUIN, P. TASDEMIR, E. PIERRON, G. TROULINAKI, K. TAVERNARAKIS, N. HICKMAN, J. A. GENESTE, O. \& KROEMER, G. (2007). Functional and physical interaction between $\mathrm{Bcl}-\mathrm{X}(\mathrm{L})$ and a $\mathrm{BH} 3-$ like domain in Beclin-1. Embo J 26(10), 2527-2539.

- MAIURI, M. C. MALIK, S. A. MORSELLI, E. KEPP, O. CRIOLLO, A. MOUCHEL, P. L. CARNUCCIO, R. \& KROEMER, G. (2009). Stimulation of autophagy by the p53 target gene Sestrin2. Cell Cycle 8(10), 1571-1576.

- MARINO, G. SAlVAdOR-MONTOliU, N. FUEYO, A. KNECHT, E. MIZUSHIMA, N. \& LOPEZ-OTIN, C. (2007). Tissue-specific autophagy alterations and increased tumorigenesis in mice deficient in Atg4C/autophagin-3. J Biol Chem 282(25), 18573-18583.

- MARTIN, S. J. \& GREEN, D. R. (1995). Protease activation during apoptosis: death by a thousand cuts? Cell 82(3), 349-352.

- MARTINEZ-VICENTE, M. TALlOCZY, Z. WONG, E. TANG, G. KOGA, H. KAUSHIK, S. DE VRIES, R. ARIAS, E. HARRIS, S. SULZER, D. \& CUERVO, A. M. (2010). Cargo recognition failure is responsible for inefficient autophagy in Huntington's disease. Nat Neurosci 13(5), 567-576.

- MARTINON, F. GAIDE, O. PETRILLI, V. MAYOR, A. \& TSCHOPP, J. (2007). NALP inflammasomes: a central role in innate immunity. Semin Immunopathol 29(3), 213-229.

- MASSEY, A. C. KAUSHIK, S. SOVAK, G. KIFFIN, R. \& CUERVO, A. M. (2006). Consequences of the selective blockage of chaperone-mediated autophagy. Proc Natl Acad Sci U S A 103(15), 5805-5810.

- MATHEW, R. KARP, C. M. BEAUDOIN, B. VUONG, N. CHEN, G. CHEN, H. Y. BRAY, K. REDDY, A. BHANOT, G. GELINAS, C. DIPAOLA, R. S. KARANTZA-WADSWORTH, V. \& WHITE, E. (2009). Autophagy suppresses tumorigenesis through elimination of p62. Cel/ 137(6), 1062-1075.

- MATHEW, R. KONGARA, S. BEAUdOIN, B. KARP, C. M. BRAY, K. DEGENHARDT, K. CHEN, G. JIN, S. \& WHITE, E. (2007). Autophagy suppresses tumor progression by limiting chromosomal instability. Genes Dev 21(11), 1367-1381.

- MATSUDA, A. SUZUKI, Y. HONDA, G. MURAMATSU, S. MATSUZAKI, O. NAGANO, Y. DOI, T. SHIMOTOHNO, K. HARADA, T. NISHIDA, E. HAYASHI, H. \& SUGANO, S. (2003). Large-scale identification and characterization of human genes that activate NF-kappaB and MAPK signaling pathways. Oncogene 22(21), 3307-3318.

- MATSUI, Y. TAKAGI, H. QU, X. ABDELLATIF, M. SAKODA, H. ASANO, T. LEVINE, B. \& SADOSHIMA, J. (2007). Distinct roles of autophagy in the heart during ischemia and reperfusion: roles of AMPactivated protein kinase and Beclin 1 in mediating autophagy. Circ Res 100(6), 914-922.

- MEDZHITOV, R. (2001). Toll-like receptors and innate immunity. Nat Rev Immuno/ 1(2), 135-145.

- MEIER, P. FINCH, A. \& EVAN, G. (2000). Apoptosis in development. Nature 407(6805), 796-801.

- MEYER-MORSE, N. ROBBinS, J. R. RAE, C. S. MOCHEGOVA, S. N. SWANSON, M. S. ZHAO, Z. VIRGIN, H. W. \& PORTNOY, D. (2010). Listeriolysin O is necessary and sufficient to induce autophagy during Listeria monocytogenes infection. PLoS One 5(1), e8610.

- MIJALJICA, D. PRESCOTT, M. KLIONSKY, D. J. \& DEVENISH, R. J. (2007). Autophagy and vacuole homeostasis: a case for self-degradation? Autophagy 3(5), 417-421.

- MILleR, B. C. ZHAO, Z. STEPhENSON, L. M. CADWELL, K. PUA, H. H. LEE, H. K. MIZUSHIMA, N. N. IWASAKI, A. HE, Y. W. SWAT, W. \& VIRGIN, H. W. T. (2008). The autophagy gene ATG5 plays an essential role in B lymphocyte development. Autophagy 4(3), 309-314.

- MIZUShIMA, N. LEVINE, B. CUERVO, A. M. \& KLIONSKY, D. J. (2008). Autophagy fights disease through cellular self-digestion. Nature 451(7182), 1069-1075.

- MIZUSHIMA, N. YAMAMOTO, A. HATANO, M. KOBAYASHI, Y. KABEYA, Y. SUZUKI, K. TOKUHISA, T. OHSUMI, Y. \& YOSHIMORI, T. (2001). Dissection of autophagosome formation using Apg5-deficient mouse embryonic stem cells. J Cell Biol 152(4), 657-668.

- MIZUSHIMA, N. YAMAMOTO, A. MATSUI, M. YOSHIMORI, T. \& OHSUMI, Y. (2004). In vivo analysis of autophagy in response to nutrient starvation using transgenic mice expressing a fluorescent autophagosome marker. Mol Biol Cel/ 15(3), 1101-1111. 
- MIZUSHIMA, N. YOSHIMORI, T. \& LEVINE, B. (2010). Methods in mammalian autophagy research. Cel/ 140(3), 313-326.

- MORSElLI, E. GALlUZZI, L. KEPP, O. VICENCIO, J. M. CRIOLLO, A. MAIURI, M. C. \& KROEMER, G. (2009). Anti- and pro-tumor functions of autophagy. Biochim Biophys Acta 1793(9), 1524-1532.

- MORTIMORE, G. E. \& SCHWORER, C. M. (1977). Induction of autophagy by amino-acid deprivation in perfused rat liver. Nature 270(5633), 174-176.

- MORVAN, J. KOCHL, R. WATSON, R. COLLINSON, L. M. JEFFERIES, H. B. \& TOOZE, S. A. (2009). In vitro reconstitution of fusion between immature autophagosomes and endosomes. Autophagy 5(5), 676-689.

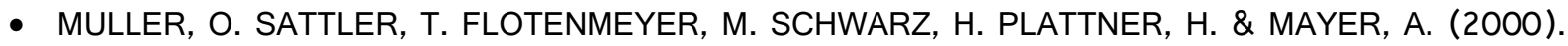
Autophagic tubes: vacuolar invaginations involved in lateral membrane sorting and inverse vesicle budding. J Cell Bio/ 151(3), 519-528.

- MUNAFO, D. B. \& COLOMBO, M. I. (2002). Induction of autophagy causes dramatic changes in the subcellular distribution of GFP-Rab24. Traffic 3(7), 472-482.

- NAGATA, S. (1997). Apoptosis by death factor. Cel/ 88(3), 355-365.

- NAKAgAWA, I. AMANO, A. MIZUSHIMA, N. YAMAMOTO, A. YAMAGUCHI, H. KAMIMOTO, T. NARA, A. FUNAO, J. NAKATA, M. TSUDA, K. HAMADA, S. \& YOSHIMORI, T. (2004). Autophagy defends cells against invading group A Streptococcus. Science 306(5698), 1037-1040.

- NAKANO, K. \& VOUSDEN, K. H. (2001). PUMA, a novel proapoptotic gene, is induced by p53. Mol Cell 7(3), 683-694.

- NARA, A. MIZUSHIMA, N. YAMAMOTO, A. KABEYA, Y. OHSUMI, Y. \& YOSHIMORI, T. (2002). SKD1 AAA ATPase-dependent endosomal transport is involved in autolysosome formation. Cell Struct Funct 27(1), 29-37.

- NEDJIC, J. AICHINGER, M. EMMERICH, J. MIZUSHIMA, N. \& KLEIN, L. (2008). Autophagy in thymic epithelium shapes the T-cell repertoire and is essential for tolerance. Nature 455(7211), 396-400.

- NISHIDA, K. KYOI, S. YAMAGUCHI, O. SADOSHIMA, J. \& OTSU, K. (2009a). The role of autophagy in the heart. Cell Death Differ 16(1), 31-38.

- NISHIDA, Y. ARAKAWA, S. FUJITANI, K. YAMAGUCHI, H. MIZUTA, T. KANASEKI, T. KOMATSU, M. OTSU, K. TSUJIMOTO, Y. \& SHIMIZU, S. (2009b). Discovery of Atg5/Atg7-independent alternative macroautophagy. Nature 461(7264), 654-658.

- O'CONNOR, L. STRASSER, A. O'REILLY, L. A. HAUSMANN, G. ADAMS, J. M. CORY, S. \& HUANG, D. C. (1998). Bim: a novel member of the Bcl-2 family that promotes apoptosis. Embo J 17(2), 384395.

- OBerstein, A. JeffrReY, P. D. \& SHI, Y. (2007). Crystal structure of the Bcl-XL-Beclin 1 peptide complex: Beclin 1 is a novel BH3-only protein. J Biol Chem 282(17), 13123-13132.

- OgAWA, M. YOSHIMORI, T. SUZUKI, T. SAGARA, H. MIZUSHIMA, N. \& SASAKAWA, C. (2005). Escape of intracellular Shigella from autophagy. Science 307(5710), 727-731.

- OlTVAI, Z. N. MILLIMAN, C. L. \& KORSMEYER, S. J. (1993). Bcl-2 heterodimerizes in vivo with a conserved homolog, Bax, that accelerates programmed cell death. Cel/ 74(4), 609-619.

- ORSI, A. POLSON, H. E. \& TOOZE, S. A. (2010). Membrane trafficking events that partake in autophagy. Curr Opin Cell Biol 22(2), 150-156.

- ORVEDAHL, A. ALEXANDER, D. TALLOCZY, Z. SUN, Q. WEI, Y. ZHANG, W. BURNS, D. LEIB, D. A. \& LEVINE, B. (2007). HSV-1 ICP34.5 confers neurovirulence by targeting the Beclin 1 autophagy protein. Cell Host Microbe 1(1), 23-35.

- ORVEDAHL, A. \& LEVINE, B. (2009). Autophagy in Mammalian antiviral immunity. Curr Top Microbiol Immunol 335, 267-285.

- ORVEDAHL, A. MACPHERSON, S. SUMPTER, R., JR. TALLOCZY, Z. ZOU, Z. \& LEVINE, B. (2010). Autophagy protects against Sindbis virus infection of the central nervous system. Cell Host Microbe 7(2), 115-127. 
- PANDEY, K. N. (2009). Functional roles of short sequence motifs in the endocytosis of membrane receptors. Front Biosci 14, 5339-5360.

- PANKIV, S. Clausen, t. H. lamark, t. BReCH, A. BRUUn, J. A. OUtZen, H. OVERVATN, A. BJORKOY, G. \& JOHANSEN, T. (2007). p62/SQSTM1 binds directly to Atg8/LC3 to facilitate degradation of ubiquitinated protein aggregates by autophagy. J Biol Chem 282(33), 24131-24145.

- PAttingRe, S. tASSA, A. QU, X. GARUti, R. LIANG, X. H. MIZUShima, N. PACKeR, M. SCHNEIDER, M. D. \& LEVINE, B. (2005). Bcl-2 antiapoptotic proteins inhibit Beclin 1-dependent autophagy. Cell 122(6), 927-939.

- POLAGER, S. \& GINSBERG, D. (2009). p53 and E2f: partners in life and death. Nat Rev Cancer $9(10), 738-748$.

- PUA, H. H. \& HE, Y. W. (2007). Maintaining T lymphocyte homeostasis: another duty of autophagy. Autophagy 3(3), 266-267.

- QU, X. YU, J. BHAGAT, G. FURUYA, N. HIBSHOOSH, H. TROXEL, A. ROSEN, J. ESKELINEN, E. L. MIZUSHIMA, N. OHSUMI, Y. CATTORETTI, G. \& LEVINE, B. (2003). Promotion of tumorigenesis by heterozygous disruption of the beclin 1 autophagy gene. J Clin Invest 112(12), 1809-1820.

- QU, X. ZOU, Z. SUN, Q. LUBY-PHELPS, K. CHENG, P. HOGAN, R. N. GILPIN, C. \& LEVINE, B. (2007). Autophagy gene-dependent clearance of apoptotic cells during embryonic development. Cell 128(5), 931-946.

- RABINOWITZ, J. D. \& WHITE, E. (2010). Autophagy and metabolism. Science 330(6009), 13441348.

- RASHMi, R. PILlai, S. G. VIJAYAlingam, S. RYERSE, J. \& CHINNADURAI, G. (2008). BH3-only protein BIK induces caspase-independent cell death with autophagic features in Bcl-2 null cells. Oncogene 27(10), 1366-1375.

- RAVIKUMAR, B. DUDEN, R. \& RUBINSZTEIN, D. C. (2002). Aggregate-prone proteins with polyglutamine and polyalanine expansions are degraded by autophagy. Hum Mol Genet 11(9), 11071117.

- RAVIKUMAR, B. IMARISIO, S. SARKAR, S. O'KANE, C. J. \& RUBINSZTEIN, D. C. (2008). Rab5 modulates aggregation and toxicity of mutant huntingtin through macroautophagy in cell and fly models of Huntington disease. J Cell Sci 121(Pt 10), 1649-1660.

- RAVIKUMaR, B. MOREAU, K. JAHREISS, L. PURI, C. \& RUBINSZTEIN, D. C. (2010). Plasma membrane contributes to the formation of pre-autophagosomal structures. Nat Cell Biol 12 (8), 747-757.

- RaVIKUmar, B. VACHER, C. BERger, Z. DAVIES, J. E. LUO, S. OROZ, L. G. SCARAVILLI, F. EASTON, D. F. DUDEN, R. O'KANE, C. J. \& RUBINSZTEIN, D. C. (2004). Inhibition of mTOR induces autophagy and reduces toxicity of polyglutamine expansions in fly and mouse models of Huntington disease. Nat Genet 36(6), 585-595.

- RAY, C. A. BLACK, R. A. KRONHEIM, S. R. GREENSTREET, T. A. SLEATH, P. R. SALVESEN, G. S. \& PICKUP, D. J. (1992). Viral inhibition of inflammation: cowpox virus encodes an inhibitor of the interleukin-1 beta converting enzyme. Cell 69(4), 597-604.

- REGGIORI, F. SHINTANI, T. NAIR, U. \& KLIONSKY, D. J. (2005). Atg9 cycles between mitochondria and the pre-autophagosomal structure in yeasts. Autophagy 1(2), 101-109.

- RENNA, M. JIMENEZ-SANCHEZ, M. SARKAR, S. \& RUBINSZTEIN, D. C. (2010). Chemical inducers of autophagy that enhance the clearance of mutant proteins in neurodegenerative diseases. $J$ Biol Chem 285(15), 11061-11067.

- ROBERTS, P. MOSHITCH-MOSHKOVITZ, S. KVAM, E. O'TOOLE, E. WINEY, M. \& GOLDFARB, D. S. (2003). Piecemeal microautophagy of nucleus in Saccharomyces cerevisiae. Mol Biol Cell 14(1), 129141.

- ROCCIO, M. BOS, J. L. \& ZWARTKRUIS, F. J. (2006). Regulation of the small GTPase Rheb by amino acids. Oncogene 25(5), 657-664.

- ROHN, W. M. ROUILLE, Y. WAGURI, S. \& HOFLACK, B. (2000). Bi-directional trafficking between the trans-Golgi network and the endosomal/lysosomal system. J Cell Sci 113 ( Pt 12), 2093-2101. 
- Ropolo, A. grasso, D. pardo, R. Sacchetti, M. L. archange, C. lo Re, A. SeuX, M. NOWAK, J. GONZALEZ, C. D. IOVANNA, J. L. \& VACCARO, M. I. (2007). The pancreatitis-induced vacuole membrane protein 1 triggers autophagy in mammalian cells. J Biol Chem 282(51), 3712437133.

- RUBINSZTEIN, D. C. CUERVO, A. M. RAVIKUMAR, B. SARKAR, S. KOROLCHUK, V. KAUSHIK, S. \& KLIONSKY, D. J. (2009). In search of an "autophagomometer". Autophagy 5(5), 585-589.

- SABBAH, A. CHANG, T. H. HARNACK, R. FROHLICH, V. TOMINAGA, K. DUBE, P. H. XIANG, Y. \& BOSE, S. (2009). Activation of innate immune antiviral responses by Nod2. Nat Immuno/ 10(10), 10731080.

- SAFTIG, P. BEERTSEN, W. \& ESKELINEN, E. L. (2008). LAMP-2: a control step for phagosome and autophagosome maturation. Autophagy 4(4), 510-512.

- SAITOH, T. \& AKIRA, S. (2010). Regulation of innate immune responses by autophagy-related proteins. $J$ Cell Biol 189(6), 925-935.

- SAMARA, C. SYNTICHAKI, P. \& TAVERNARAKIS, N. (2008). Autophagy is required for necrotic cell death in Caenorhabditis elegans. Cell Death Differ 15(1), 105-112.

- SANJUAN, M. A. DILLON, C. P. TAIT, S. W. MOSHIACH, S. DORSEY, F. CONNELL, S. KOMATSU, M. TANAKA, K. CLEVELAND, J. L. WITHOFF, S. \& GREEN, D. R. (2007). Toll-like receptor signalling in macrophages links the autophagy pathway to phagocytosis. Nature 450(7173), 1253-1257.

- SARKAR, S. KRISHNA, G. IMARISIO, S. SAIKI, S. O'KANE, C. J. \& RUBINSZTEIN, D. C. (2008). A rational mechanism for combination treatment of Huntington's disease using lithium and rapamycin. Hum Mol Genet 17 (2), 170-178.

- SARKAR, S. PERLSTEIN, E. O. IMARISIO, S. PINEAU, S. CORDENIER, A. MAGLATHLIN, R. L. WEBSTER, J. A. LEWIS, T. A. O'KANE, C. J. SCHREIBER, S. L. \& RUBINSZTEIN, D. C. (2007). Small molecules enhance autophagy and reduce toxicity in Huntington's disease models. Nat Chem Biol $3(6), 331-338$.

- SAVILL, J. \& FADOK, V. (2000). Corpse clearance defines the meaning of cell death. Nature 407(6805), 784-788.

- SCARLATTI, F. MAFFEI, R. BEAU, I. CODOGNO, P. \& GHIDONI, R. (2008). Role of non-canonical Beclin 1-independent autophagy in cell death induced by resveratrol in human breast cancer cells. Cell Death Differ 15(8), 1318-1329.

- SCOTT, R. C. JUHASZ, G. \& NEUFELD, T. P. (2007). Direct induction of autophagy by Atg1 inhibits cell growth and induces apoptotic cell death. Curr Biol 17(1), 1-11.

- SCOTT, S. V. BABA, M. OHSUMI, Y. \& KLIONSKY, D. J. (1997). Aminopeptidase I is targeted to the vacuole by a nonclassical vesicular mechanism. J Cell Biol 138(1), 37-44.

- SCHMELZLE, T. \& HALL, M. N. (2000). TOR, a central controller of cell growth. Cell 103(2), 253262.

- SCHOBEL, S. NEUMANN, S. SEED, B. \& LICHTENTHALER, S. F. (2006). Expression cloning screen for modifiers of amyloid precursor protein shedding. Int $J$ Dev Neurosci 24(2-3), 141-148.

- SHACKA, J. J. KLOCKE, B. J. SHIBATA, M. UCHIYAMA, Y. DATTA, G. SCHMIDT, R. E. \& ROTH, K. A. (2006). Bafilomycin A1 inhibits chloroquine-induced death of cerebellar granule neurons. Mol Pharmacol 69(4), 1125-1136.

- SHAO, Y. GAO, Z. MARKS, P. A. \& JIANG, X. (2004). Apoptotic and autophagic cell death induced by histone deacetylase inhibitors. Proc Natl Acad Sci U S A 101(52), 18030-18035.

- SHAW, P. J. LAMKANFI, M. \& KANNEGANTI, T. D. (2010). NOD-like receptor (NLR) signaling beyond the inflammasome. Eur J Immunol 40(3), 624-627.

- SHIMIZU, S. KANASEKI, T. MIZUSHIMA, N. MIZUTA, T. ARAKAWA-KOBAYASHI, S. THOMPSON, C. B. \& TSUJIMOTO, Y. (2004). Role of Bcl-2 family proteins in a non-apoptotic programmed cell death dependent on autophagy genes. Nat Cell Biol 6(12), 1221-1228.

- SHVETS, E. FASS, E. \& ELAZAR, Z. (2008). Utilizing flow cytometry to monitor autophagy in living mammalian cells. Autophagy 4(5), 621-628. 
- SINGH, S. B. DAVIS, A. S. TAYLOR, G. A. \& DERETIC, V. (2006). Human IRGM induces autophagy to eliminate intracellular mycobacteria. Science 313(5792), 1438-1441.

- SONGYANG, Z. FANNING, A. S. FU, C. XU, J. MARFATIA, S. M. CHISHTI, A. H. CROMPTON, A. CHAN, A. C. ANDERSON, J. M. \& CANTLEY, L. C. (1997). Recognition of unique carboxyl-terminal motifs by distinct PDZ domains. Science 275(5296), 73-77.

- SOU, Y. S. WAGURI, S. IWATA, J. UENO, T. FUJIMURA, T. HARA, T. SAWADA, N. YAMADA, A. MIZUSHIMA, N. UCHIYAMA, Y. KOMINAMI, E. TANAKA, K. \& KOMATSU, M. (2008). The Atg8 conjugation system is indispensable for proper development of autophagic isolation membranes in mice. Mol Biol Cell 19(11), 4762-4775.

- SPERANDio, S. DE BELLE, I. \& BREDESEN, D. E. (2000). An alternative, nonapoptotic form of programmed cell death. Proc Natl Acad Sci U S A 97(26), 14376-14381.

- STEIN, T. D. ANDERS, N. J. DECARLI, C. CHAN, S. L. MATTSON, M. P. \& JOHNSON, J. A. (2004). Neutralization of transthyretin reverses the neuroprotective effects of secreted amyloid precursor protein (APP) in APPSW mice resulting in tau phosphorylation and loss of hippocampal neurons: support for the amyloid hypothesis. J Neurosci 24(35), 7707-7717.

- SUMPTER, R., JR. \& LEVINE, B. (2010). Autophagy and innate immunity: triggering, targeting and tuning. Semin Cell Dev Biol 21(7), 699-711.

- SUZUKI, K. KIRISAKO, T. KAMADA, Y. MIZUSHIMA, N. NODA, T. \& OHSUMI, Y. (2001). The preautophagosomal structure organized by concerted functions of APG genes is essential for autophagosome formation. Embo J 20(21), 5971-5981.

- TAKAHASHI, Y. COPPOLA, D. MATSUSHITA, N. CUALING, H. D. SUN, M. SATO, Y. LIANG, C. JUNG, J. U. CHENG, J. Q. MULE, J. J. PLEDGER, W. J. \& WANG, H. G. (2007). Bif-1 interacts with Beclin 1 through UVRAG and regulates autophagy and tumorigenesis. Nat Cell Bio/ 9(10), 1142-1151.

- TAKEUCHI, O. \& AKIRA, S. (2001). Toll-like receptors; their physiological role and signal transduction system. Int Immunopharmacol 1(4), 625-635.

- TAKEUCHI, O. \& AKIRA, S. (2007). Recognition of viruses by innate immunity. Immunol Rev 220, 214-224.

- TALLOCZY, Z. VIRGIN, H. W. T. \& LEVINE, B. (2006). PKR-dependent autophagic degradation of herpes simplex virus type 1. Autophagy 2(1), 24-29.

- TAMAI, K. TANAKA, N. NARA, A. YAMAMOTO, A. NAKAGAWA, I. YOSHIMORI, T. UENO, Y. SHIMOSEGAWA, T. \& SUGAMURA, K. (2007). Role of Hrs in maturation of autophagosomes in mammalian cells. Biochem Biophys Res Commun 360(4), 721-727.

- TANAKA, Y. GUHDE, G. SUTER, A. ESKELINEN, E. L. HARTMANN, D. LULLMANN-RAUCH, R. JANSSEN, P. M. BLANZ, J. VON FIGURA, K. \& SAFTIG, P. (2000). Accumulation of autophagic vacuoles and cardiomyopathy in LAMP-2-deficient mice. Nature 406(6798), 902-906.

- TANIDA, I. MINEMATSU-IKEGUCHI, N. UENO, T. \& KOMINAMI, E. (2005). Lysosomal turnover, but not a cellular level, of endogenous LC3 is a marker for autophagy. Autophagy 1(2), 84-91.

- TANIDA, I. YAMAJI, T. UENO, T. ISHIURA, S. KOMINAMI, E. \& HANADA, K. (2008). Consideration about negative controls for LC3 and expression vectors for four colored fluorescent protein-LC3 negative controls. Autophagy 4(1), 131-134.

- TANIGUCHI, T. \& TAKAOKA, A. (2002). The interferon-alpha/beta system in antiviral responses: a multimodal machinery of gene regulation by the IRF family of transcription factors. Curr Opin Immunol 14(1), 111-116.

- tASDemiR, E. MAIURI, M. C. GalluZZI, L. VitAle, I. DJAVAHERI-MERGNy, M. D'AMELIO, M. CRIOLLO, A. MORSELLI, E. ZHU, C. HARPER, F. NANNMARK, U. SAMARA, C. PINTON, P. VICENCIO, J. M. CARNUCCIO, R. MOLL, U. M. MADEO, F. PATERLINI-BRECHOT, P. RIZZUTO, R. SZABADKAI, G. PIERRON, G. BLOMGREN, K. TAVERNARAKIS, N. CODOGNO, P. CECCONI, F. \& KROEMER, G. (2008). Regulation of autophagy by cytoplasmic p53. Nat Cell Bio/ 10(6), 676-687.

- TERMAN, A. (1995). The effect of age on formation and elimination of autophagic vacuoles in mouse hepatocytes. Gerontology 41 Suppl 2, 319-326. 
- THOMPSON, C. B. (1995). Apoptosis in the pathogenesis and treatment of disease. Science 267(5203), 1456-1462.

- THOREEN, C. C. KANG, S. A. CHANG, J. W. LIU, Q. ZHANG, J. GAO, Y. REICHLING, L. J. SIM, T. SABATINI, D. M. \& GRAY, N. S. (2009). An ATP-competitive mammalian target of rapamycin inhibitor reveals rapamycin-resistant functions of mTORC1. J Biol Chem 284(12), 8023-8032.

- TIAN, S. LIN, J. JUN ZHOU, J. WANG, X. LI, Y. REN, X. YU, W. ZHONG, W. XIAO, J. SHENG, F. CHEN, Y. JIN, C. LI, S. ZHENG, Z. \& XIA, B. (2010). Beclin 1-independent autophagy induced by a $\mathrm{Bcl}-\mathrm{XL} / \mathrm{Bcl}-2$ targeting compound, Z18. Autophagy 6(8), 1032-1041.

- TODDE, V. VEENHUIS, M. \& VAN DER KLEI, I. J. (2009). Autophagy: principles and significance in health and disease. Biochim Biophys Acta 1792(1), 3-13.

- TOOZE, J. HOLLINSHEAD, M. LUDWIG, T. HOWELL, K. HOFLACK, B. \& KERN, H. (1990). In exocrine pancreas, the basolateral endocytic pathway converges with the autophagic pathway immediately after the early endosome. J Cell Bio/111(2), 329-345.

- TOTH, M. L. SIGMOND, T. BORSOS, E. BARNA, J. ERDELYI, P. TAKACS-VELLAI, K. OROSZ, L. KOVACS, A. L. CSIKOS, G. SASS, M. \& VELLAI, T. (2008). Longevity pathways converge on autophagy genes to regulate life span in Caenorhabditis elegans. Autophagy 4(3), 330-338.

- travassos, L. H. CARneiro, L. A. RAMJeEt, M. hussey, S. KIM, Y. G. MAGAlhaES, J. G. YUAN, L. SOARES, F. CHEA, E. LE BOURHIS, L. BONECA, I. G. ALLAOUI, A. JONES, N. L. NUNEZ, G. GIRARDIN, S. E. \& PHILPOTT, D. J. (2010). Nod1 and Nod2 direct autophagy by recruiting ATG16L1 to the plasma membrane at the site of bacterial entry. Nat Immuno/ 11(1), 55-62.

- TWIG, G. EloRZA, A. MOLINA, A. J. MOHAMED, H. WIKSTROM, J. D. WALZER, G. STILES, L. HAIGH, S. E. KATZ, S. LAS, G. ALROY, J. WU, M. PY, B. F. YUAN, J. DEENEY, J. T. CORKEY, B. E. \& SHIRIHAI, O. S. (2008). Fission and selective fusion govern mitochondrial segregation and elimination by autophagy. Embo $J 27(2), 433-446$.

- ULLRICH, S. MUNCH, A. NEUMANN, S. KREMMER, E. TATZELT, J. \& LICHTENTHALER, S. F. (2010). The novel membrane protein TMEM59 modulates complex glycosylation, cell surface expression, and secretion of the amyloid precursor protein. J Biol Chem 285(27), 20664-20674.

- UTTENWEILER, A. SCHWARZ, H. \& MAYER, A. (2005). Microautophagic vacuole invagination requires calmodulin in a Ca2+-independent function. J Biol Chem 280(39), 33289-33297.

- VAN DER VAART, A. GRIFFITH, J. \& REGGIORI, F. (2010). Exit from the Golgi Is Required for the Expansion of the Autophagosomal Phagophore in Yeast Saccharomyces cerevisiae. Mol Biol Cell.

- VANDE VELDE, C. CIZEAU, J. DUBIK, D. ALIMONTI, J. BROWN, T. ISRAELS, S. HAKEM, R. \& GREENBERG, A. H. (2000). BNIP3 and genetic control of necrosis-like cell death through the mitochondrial permeability transition pore. Mol Cell Biol 20(15), 5454-5468.

- VAZQUEZ-MARTIN, A. OliVERAS-FERRAROS, C. \& MENENDEZ, J. A. (2009). Autophagy facilitates the development of breast cancer resistance to the anti-HER2 monoclonal antibody trastuzumab. PLoS One 4(7), e6251.

- VOGELSTEIN, B. LANE, D. \& LEVINE, A. J. (2000). Surfing the p53 network. Nature 408(6810), 307-310.

- WANG, K. YIN, X. M. CHAO, D. T. MILLIMAN, C. L. \& KORSMEYER, S. J. (1996). BID: a novel BH3 domain-only death agonist. Genes Dev 10(22), 2859-2869.

- WEI, M. C. ZONG, W. X. CHENG, E. H. LINDSTEN, T. PANOUTSAKOPOULOU, V. ROSS, A. J. ROTH, K. A. MACGREGOR, G. R. THOMPSON, C. B. \& KORSMEYER, S. J. (2001). Proapoptotic BAX and BAK: a requisite gateway to mitochondrial dysfunction and death. Science 292(5517), 727730.

- WEI, Y. PATTINGRE, S. SINHA, S. BASSIK, M. \& LEVINE, B. (2008). JNK1-mediated phosphorylation of $\mathrm{Bcl}-2$ regulates starvation-induced autophagy. $\mathrm{Mol} \mathrm{Cell} 30(6), 678-688$.

- WEIDBERG, H. SHVETS, E. SHPILKA, T. SHIMRON, F. SHINDER, V. \& ELAZAR, Z. (2010). LC3 and GATE-16/GABARAP subfamilies are both essential yet act differently in autophagosome biogenesis. Embo J 29(11), 1792-1802. 
- WILliams, A. SARKAR, S. CUDDON, P. TTOFI, E. K. SAIKI, S. SIDDIQI, F. H. JAHREISS, L. FLEMING, A. PASK, D. GOLDSMITH, P. O'KANE, C. J. FLOTO, R. A. \& RUBINSZTEIN, D. C. (2008). Novel targets for Huntington's disease in an mTOR-independent autophagy pathway. Nat Chem Biol 4(5), 295-305.

- WULLSCHLEGER, S. LOEWITH, R. \& HALL, M. N. (2006). TOR signaling in growth and metabolism. Cell 124(3), 471-484.

- XU, Y. JAGANNATH, C. LIU, X. D. ShARAFKHANEH, A. KOlODZIEJSKA, K. E. \& EISSA, N. T. (2007). Toll-like receptor 4 is a sensor for autophagy associated with innate immunity. Immunity $27(1)$, 135-144.

- yAMAMOto, A. TAGAWA, Y. YOSHIMORI, T. MORIYAMA, Y. MASAKI, R. \& TASHIRO, Y. (1998). Bafilomycin A1 prevents maturation of autophagic vacuoles by inhibiting fusion between autophagosomes and lysosomes in rat hepatoma cell line, H-4-II-E cells. Cell Struct Funct 23(1), 33-42.

- YANG, Z. \& KLIONSKY, D. J. (2010). Mammalian autophagy: core molecular machinery and signaling regulation. Curr Opin Cell Biol 22(2), 124-131.

- YANKNER, B. A. DUFFY, L. K. \& KIRSCHNER, D. A. (1990). Neurotrophic and neurotoxic effects of amyloid beta protein: reversal by tachykinin neuropeptides. Science 250(4978), 279-282.

- YLA-ANTTILA, P. VIHINEN, H. JOKITALO, E. \& ESKELINEN, E. L. (2009). Monitoring autophagy by electron microscopy in Mammalian cells. Methods Enzymol 452, 143-164.

- YONISH-ROUACH, E. RESNITZKY, D. LOTEM, J. SACHS, L. KIMCHI, A. \& OREN, M. (1991). Wildtype p53 induces apoptosis of myeloid leukaemic cells that is inhibited by interleukin-6. Nature 352(6333), 345-347.

- YOSHIKAWA, Y. OGAWA, M. HAIN, T. YOSHIDA, M. FUKUMATSU, M. KIM, M. MIMURO, H. NAKAGAWA, I. YANAGAWA, T. ISHII, T. KAKIZUKA, A. SZTUL, E. CHAKRABORTY, T. \& SASAKAWA, C. (2009). Listeria monocytogenes ActA-mediated escape from autophagic recognition. Nat Cell Biol 11(10), 1233-1240.

- YOSHIMORI, T. \& NODA, T. (2008). Toward unraveling membrane biogenesis in mammalian autophagy. Curr Opin Cell Bio/ 20(4), 401-407.

- YOUSEFI, S. PEROZZO, R. SCHMID, I. ZIEMIECKI, A. SCHAFFNER, T. SCAPOZZA, L. BRUNNER, T. \& SIMON, H. U. (2006). Calpain-mediated cleavage of Atg5 switches autophagy to apoptosis. Nat Cell $\mathrm{Biol}$ 8(10), 1124-1132.

- YU, J. ZHANG, L. HWANG, P. M. KINZLER, K. W. \& VOGELSTEIN, B. (2001). PUMA induces the rapid apoptosis of colorectal cancer cells. $\mathrm{Mol} \mathrm{Cell}$ 7(3), 673-682.

- YU, L. AlVA, A. SU, H. DUTT, P. FREUNDT, E. WELSH, S. BAEHRECKE, E. H. \& LENARDO, M. J. (2004). Regulation of an ATG7-beclin 1 program of autophagic cell death by caspase-8. Science 304(5676), 1500-1502.

- YU, L. WAN, F. DUTTA, S. WELSH, S. LIU, Z. FREUNDT, E. BAEHRECKE, E. H. \& LENARDO, M. (2006). Autophagic programmed cell death by selective catalase degradation. Proc Natl Acad Sci U S A 103(13), 4952-4957.

- YU, W. H. CUERVO, A. M. KUMAR, A. PETERHOFF, C. M. SCHMIDT, S. D. LEE, J. H. MOHAN, P. S. MERCKEN, M. FARMERY, M. R. TJERNBERG, L. O. JIANG, Y. DUFF, K. UCHIYAMA, Y. NASLUND, J. MATHEWS, P. M. CATALDO, A. M. \& NIXON, R. A. (2005). Macroautophagy--a novel Beta-amyloid peptide-generating pathway activated in Alzheimer's disease. J Cell Biol 171(1), 87-98.

- YUE, Z. JIN, S. YANG, C. LEVINE, A. J. \& HEINTZ, N. (2003). Beclin 1, an autophagy gene essential for early embryonic development, is a haploinsufficient tumor suppressor. Proc Natl Acad Sci U S A 100(25), 15077-15082.

- ZALCKVAR, E. BERISSI, H. MIZRACHY, L. IDELCHUK, Y. KOREN, I. EISENSTEIN, M. SABANAY, H. PINKAS-KRAMARSKI, R. \& KIMCHI, A. (2009). DAP-kinase-mediated phosphorylation on the BH3 domain of beclin 1 promotes dissociation of beclin 1 from $\mathrm{Bcl}-\mathrm{XL}$ and induction of autophagy. EMBO Rep 10(3), 285-292. 
- ZHANG, L. YU, J. PAN, H. HU, P. HAO, Y. CAI, W. ZHU, H. YU, A. D. XIE, X. MA, D. \& YUAN, J. (2007). Small molecule regulators of autophagy identified by an image-based high-throughput screen. Proc Natl Acad Sci U S A 104(48), 19023-19028.

- ZHENG, Y. T. SHAHNAZARI, S. BRECH, A. LAMARK, T. JOHANSEN, T. \& BRUMELL, J. H. (2009). The adaptor protein p62/SQSTM1 targets invading bacteria to the autophagy pathway. $J$ Immunol 183(9), 5909-5916.

- ZHOU, D. LI, P. LIN, Y. LOTT, J. M. HISLOP, A. D. CANADAY, D. H. BRUTKIEWICZ, R. R. \& BLUM, J. S. (2005). Lamp-2a facilitates MHC class II presentation of cytoplasmic antigens. Immunity 22(5), 571-581. 




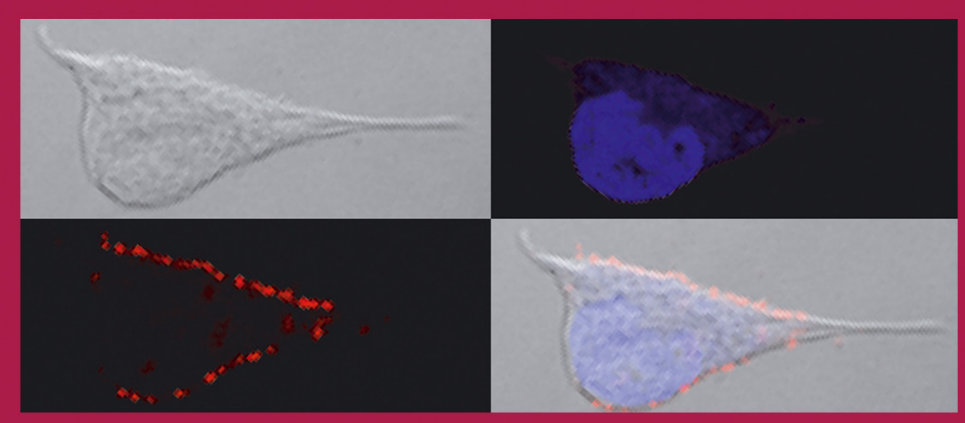

